San Jose State University

SJSU ScholarWorks

Master's Theses

Master's Theses and Graduate Research

Summer 2016

\title{
Testing Camera Trap Density Estimates From the Spatial Capture Model and Calibrated Capture Rate Indices Against Kangaroo Rat (Dipodomys spp.) Live Trapping Data
}

Timothy A. Walker

San Jose State University

Follow this and additional works at: https://scholarworks.sjsu.edu/etd_theses

\section{Recommended Citation}

Walker, Timothy A., "Testing Camera Trap Density Estimates From the Spatial Capture Model and Calibrated Capture Rate Indices Against Kangaroo Rat (Dipodomys spp.) Live Trapping Data" (2016). Master's Theses. 4742.

DOI: https://doi.org/10.31979/etd.tufx-42s4

https://scholarworks.sjsu.edu/etd_theses/4742

This Thesis is brought to you for free and open access by the Master's Theses and Graduate Research at SJSU ScholarWorks. It has been accepted for inclusion in Master's Theses by an authorized administrator of SJSU ScholarWorks. For more information, please contact scholarworks@sjsu.edu. 
TESTING CAMERA TRAP DENSITY ESTIMATES FROM THE SPATIAL CAPTURE MODEL AND CALIBRATED CAPTURE RATE INDICES AGAINST KANGAROO RAT (DIPODOMYS SPP.) LIVE TRAPPING DATA

\author{
A Thesis \\ Presented to \\ The Faculty of the Department of Biological Sciences \\ San José State University \\ In Partial Fulfillment \\ of the Requirements for the Degree \\ Master of Science
}

by

Timothy A. Walker

August 2016 
(C) 2016

Timothy A. Walker

ALL RIGHTS RESERVED 
The Designated Thesis Committee Approves the Thesis Titled

TESTING CAMERA TRAP DENSITY ESTIMATES FROM THE SPATIAL CAPTURE MODEL AND CALIBRATED CAPTURE RATE INDICES AGAINST KANGAROO RAT (DIPODOMYS SPP.) LIVE TRAPPING DATA

by

Timothy A. Walker

APPROVED FOR THE DEPARTMENT OF BIOLOGICAL SCIENCES

SAN JOSÉ STATE UNIVERSITY

August 2016

Dr. Scott Shaffer Department of Biological Sciences

Stephanie Trewhitt Department of Biological Sciences

Dr. Jerry Smith Department of Biological Sciences 


\title{
ABSTRACT \\ TESTING CAMERA TRAP DENSITY ESTIMATES FROM THE SPATIAL CAPTURE MODEL AND CALIBRATED CAPTURE RATE INDICES AGAINST KANGAROO RAT (DIPODOMYS SPP.) LIVE TRAPPING DATA
}

\author{
by Timothy A. Walker
}

Camera trapping studies often focus on estimating population density, which is critical for managing wild populations. Density estimators typically require unique markers such as stripe patterns to identify individuals but most animals do not have such markings. The spatial capture model (SC model; Chandler \& Royle, 2013) estimates density without individual identification but lacks sufficient field testing. Here, both the SC model and calibrated capture rate indices were compared against ten sessions of live trapping data on kangaroo rats (Dipodomys spp). These camera and live trapping data were combined in a joint-likelihood model to further compare the two methods. From these comparisons, the factors governing the SC model's success were scrutinized. Additionally, a method for estimating missed captures was developed and tested here. Regressions comparing live trapping density to the SC model density and capture rate were significant only for the capture rate comparison. Missed image rate had a significant relationship with ambient nighttime temperatures but only marginally improved the capture rate index calibration. Results showed the SC model was highly sensitive to deviations from its movement model, producing potentially misleading results. The model may be effective only when movement assumptions hold. Several factors such as camera coverage area, microhabitat, and burrow locations could be incorporated into the SC model density estimation process to improve precision and inference. 


\section{ACKNOWLEDGEMENTS}

First of all, I would like to thank my parents Thom and Jane Walker for all of their emotional and financial support throughout my education. I know that I would not be where I am today if it were not for their continued support, and I am eternally thankful. I would also like to thank my grandparents Bill and Betty Walker for entrusting money to my cousins and I for higher education. That gift has been one of the most important in my life, and I cannot thank them enough.

I would also like to thank the multitude of volunteers who helped me through all of my field work, without them the task would have been endlessly tiring and a lot less entertaining. I would especially like to thank Courtney Craig, Christian Lappin, Drew Stromquist, and Karissa Denny for repeatedly giving up their weekends to make my life in the field more manageable and a lot more fun.

Getting started on collecting the live trapping data was one of the hardest parts of this project and would not have been possible without Joie de Leon, Melissa Ainsworth, Celia Tarcha, and Kevin Tu crawling under dense chaparral with me in the Pinnacles heat. I would especially like to thank Daniel Corral for not only being along with me on that first trip but also for teaching me how to run a trap line on my own. I would have been lost without him there.

The help and support from Paul Johnson and Pinnacles National Park was also greatly appreciated. Pinnacles was a beautiful place to spend one weekend a month at, and Paul was crucial in keeping me safe in the field and housed at night. 
I also want to thank my entire committee for helping me every step of the way. Stephanie, for fostering my interest in conservation biology and believing that a lowly arts student could succeed in biology. Without her belief in me and the work ethic she instilled in me, I would not be where I am today. Jerry, for being an excellent example of an ethical conservation biologist, and all the help and support he gave during the earliest planning stages of my project. Finally, to Scott, who pushed me to get through piles of data analysis and helped me to focus and not get lost in the endless details of my project. I could not have completed this project without all of their support.

Finally, I would like to thank all of the little lives that were lost along the way: one pocket mouse, two kangaroo rats, five deer mice, and a harvest mouse. I tried my best to keep them warm and safe, and they have my sincerest apologies for taking their lives during the course of this project. I hope that the methodologies studied here help to reduce mortalities in the future and keep these little guys a little safer. 


\section{Table of Contents}

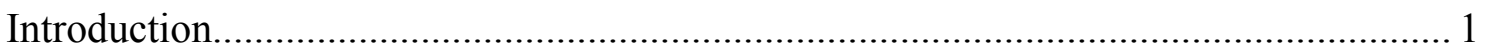

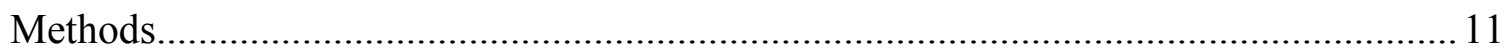

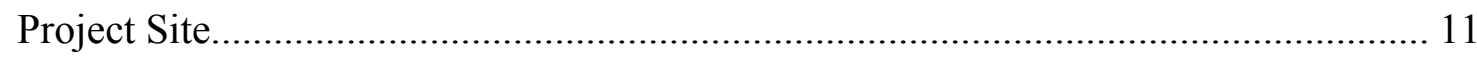

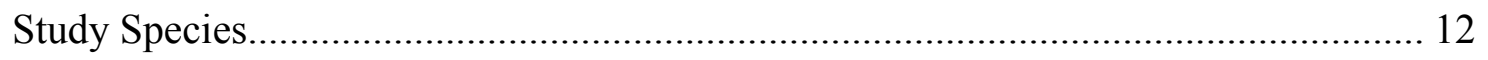

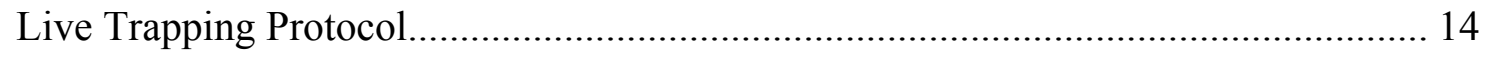

Camera Trapping Protocol................................................................................ 17

Analysis of the Calibrated Capture Rate Index and Spatial Capture Model............... 21

Spatial Capture-Recapture Analysis............................................................ 22

Analysis of the live trapping data using the multinomial model............................ 26

Analysis of the camera trapping data using the spatial capture model.................... 27

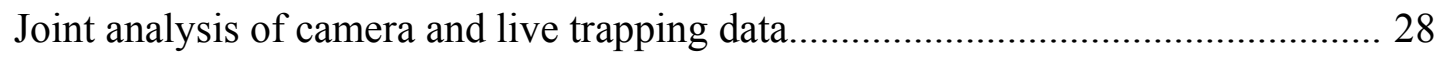

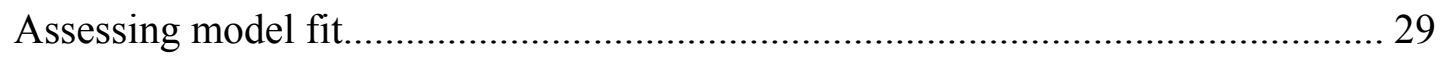

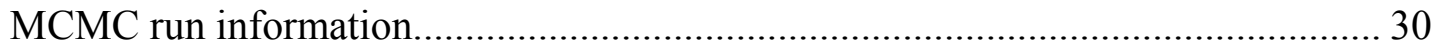

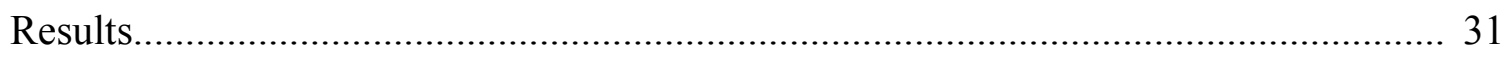

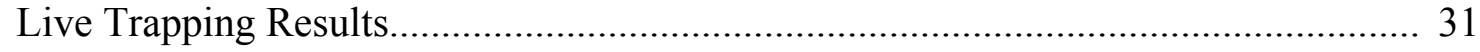

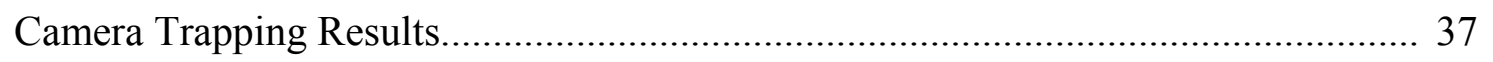

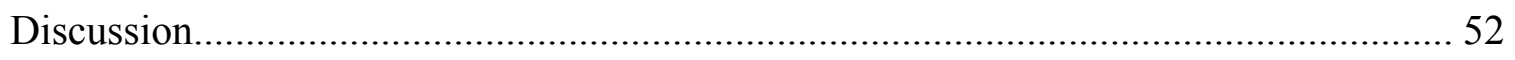

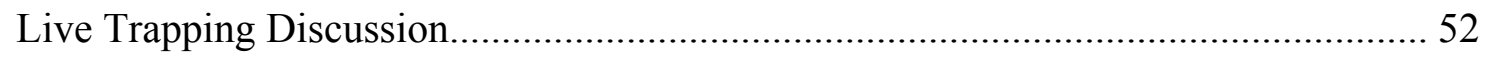

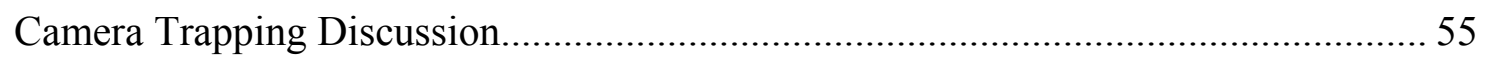

Suggestions for Future Use of the Spatial Capture Model...................................... 64

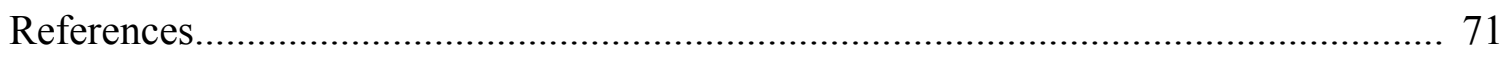




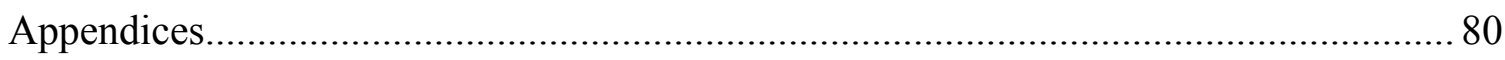

Appendix A. Capture Data and Trap Locations........................................................... 80

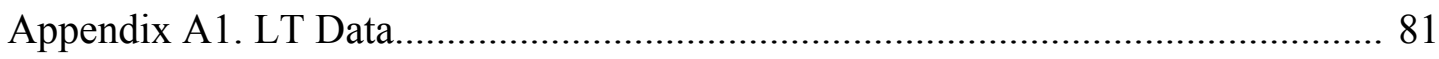

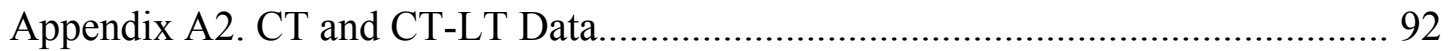

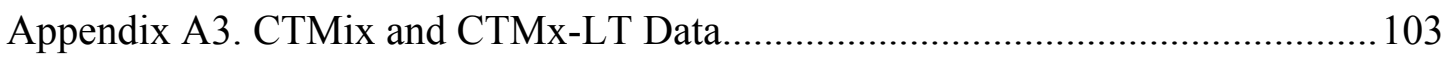

Appendix B. Model Specification.................................................................................109

Appendix C. Parameter Estimate Tables.................................................................. 121

Appendix C1. Density and Abundance for CT \& CTMix........................................ 121

Appendix C2. Home Range Radius and Capture Rate for CT \& CTMix.................. 122

Appendix C3. All Parameters for CT-LT, CTMx-LT \& LT..................................... 123

Appendix C4. Bayesian p-values for CT, CTMix, CT-LT, CTMx-LT and LT........ 124

Appendix D. Home Range Center Estimate Maps...................................................... 125

Appendix E. Home Range Center Density Plots......................................................... 151 


\section{LIST OF FIGURES}

Figure 1. Map showing the location Pinnacles National Park in California. Inset map shows the Pinnacles park boundary and the location of the project site within the park.

Figure 2. Maps showing the layout of the camera grid for all seven placements. The average layout of the live trapping grid is also show in the bottom right for reference.

Figure 3. Graph showing the detection function, $d\left(\boldsymbol{x}_{\boldsymbol{j}}, \boldsymbol{s}_{i}\right)$, set to different values of $a$. The intersection of all three variations at $z$ and $\sigma$ shows how the biologically relevant factor, $\sigma$, has the same interpretation no matter the shape

Figure $4 a-4 b$. Estimates of the home range radius parameter, $\sigma$, from the the single-session (4a) and mixed-session (4b) datasets. Bars represent modal estimates. Error bars depict upper and lower credible intervals calculated by $95 \%$ highest density interval. Anomalously high values are displayed above the figures.

Figure 5a-5c. Estimates of the multinomial capture rate parameter, $\alpha_{0}$, from the LT (5a), CT-LT (5b) and CTMx-LT (5c) datasets. Bars represent modal estimates. Error bars depict upper and lower credible intervals calculated by $95 \%$ highest density interval. Anomalously high values are displayed above the figures. Value listed is the upper credible interval

Figure 6. Bar graph comparing Bayesian p-values from the LT dataset. The dashed line shows a value of 0.5 , which is representative of good model fit

Figure 7. Bar graph showing the results of the optimal hit window analysis. For equivalent scaling across hit windows, bars heights were calculated as $e^{|\log (r)|}$, where $r$ is the variance-to-mean ratio for each hit window averaged over all sessions and cameras. Error bars depict a standard deviation of averaged variance-to-mean ratios. The bar representing the hit window with the optimal mean-tovariance ratio, $2 \mathrm{~h}$, is highlighted in light grey. 
Figure 8a-8c. Results of regressions comparing (8a) missed capture rate to average ambient nighttime temperatures, (8b) SC model density from the EP-SP sessions to LT density, and (8c) average daily capture rate to live trapping density. Solid lines indicate significant regressions coefficients, for which linear models are given. Dashed lines indicate non-significant regression coefficients.

Figure 9a-9b. Bar graphs comparing the estimates of abundance, $N$, from (9a) the CT and CT-LT datasets and (9b) the CTMix and CTMx-LT datasets. Bars represent modal estimates. Error bars depict upper and lower credible intervals calculated by $95 \%$ highest density interval

Figure 10a-10b. Bar graphs comparing the estimates of the maximum capture rate parameter, $\lambda_{0}$, from (10a) the CT and CT-LT datasets and (10b) the CTMix and CTMx-LT datasets. Bars represent modal estimates. Error bars depict upper and lower credible intervals calculated by $95 \%$ highest density interval.

Figure 11a-11b. Bar graphs comparing Bayesian p-values from (11a) the CT datasets and (11b) the CT-LT datasets. The dashed line shows a value of 0.5 , which is representative of good model fit. For the CT-LT data, separate Bayesian p-values are given for the Live trapping (LT Fit) data and the camera trapping (CT Fit) data

Figure 12a-12b. Bar graphs comparing Bayesian p-values from (12a) the CTMix datasets and (12b) the CTMx-LT datasets. The dashed line shows a value of 0.5 , which is representative of good model fit. For the CTLT data, separate Bayesian p-values are given for the Live trapping (LT Fit) data and the camera trapping (CT Fit) data....

Figure 13. Comparisons of the EP and HN detection functions graphed using modal posterior estimates from $\lambda_{0}$ and $\sigma$. The $\mathrm{Y}$-axis represents the estimated camera capture rate. The $\mathrm{X}$-axis represents the distance from a given home range center, $s_{i}$, to a given trap, $x_{j}$ (i.e., $\left.\left\|x_{j}-s_{i}\right\|\right)$. Note the alignment of the two functions in their middle sections and the differences in their peaks 


\section{LIST OF TABLES}

Table 1. List of session number and trapping dates from all datasets: LT, CT, CTLT, CTMix and CTMx-LT. All CT dates that overlap LT dates were excluded from analysis and the $\mathrm{CT}$ day count.........................................

Table 2. Summary of live trapping statistics for all species captured including: the number of sessions observed, the number of unique capture instances, total tags used, and the number of individuals that died. Sessions which were removed (R1, R2) are highlighted in dark grey. The gap in data collection is shown as a dark band

Table 3. Camera trap summary information for the most frequent species captured. Session totals are based on the $2 \mathrm{~h}$ hit window. Western harvest mice (Reithrodontomys megalotis) and North American deer mice (Peromyscus maniculatus) were difficult to differentiate in camera trap images. As such, the summary information listed for these two species is a best guess by the reviewer

Table 4. List of uncommon species captured during camera trapping that were not listed in the capture histories in Table 3

Table 5. List of summary statistics for each of the CT sessions including information on the number of triggered captures, the number of interval captures, miss rates, average temperatures, camera effort, and amount of camera outages 


\section{Introduction}

Camera traps are becoming an increasingly popular tool in wildlife ecology, and they have been used in studies to test a variety of hypotheses across a wide range of taxa. While camera trap studies have primarily focused on felids and large ungulates (Cutler \& Swann, 1999; Kucera \& Barrett, 2010), other groups, such as rodents, have also received considerable attention. (McCallum, 2012). One advantage of studying small mammals with camera traps is the ease of implementing complementary live trapping studies as efficacy trials of camera methodologies. As such, many studies have compared the two methods in a variety of study designs including presence absence (Bayrakçi, Carey \& Wilson, 2001; De Bondi, White, Stevens \& Cooke, 2010), relative abundance (Torre, Peris \& Tena, 2005), density (Villette, 2014), activity times (Pearson, 1959; Torre et al., 2005; Phillips, 2013), and occupancy (McDonald et al., 2015; Phillips, 2013; see also Rendall, Sutherland, Cooke \& White, 2014; Glen, Cockburn, Nichols, Ekanayake \& Warbutron, 2013; De Sa, Zweig, Percival, Kitchens \& Kasbohm, 2012). Estimation of population density has long been an area of focus in camera trap research (Cutler \& Swann, 1999; Kucera \& Barrett, 2010). However, camera trap studies estimating density in small mammal populations are rare (Villette, 2014), and in no study to date has it been attempted to estimate small mammal population density from camera data alone.

There has been great focus on the estimation of density in camera trap research since Ullas Karanth first used camera traps and capture-recapture (CR) techniques to estimate tiger densities (Karanth, 1995; Karanth \& Nichols, 1998; Cutler \& Swann, 1999; Kucera \& Barrett, 2010). Capture-recapture and other similar methods rely on an ability 
to uniquely identify individuals from camera trap images using marks such as stripe patterns, blemishes, and differences in coat color (Karanth, 1995; Karanth \& Nichols, 1998; Trolle \& Kéry 2003; Negroes et al., 2010; Foster \& Harmsen, 2012; Sollmann et al., 2013a; Rich et al., 2014). Unfortunately, individuals cannot be identified using these techniques for the majority of species. Yet, a non-invasive method such as camera trapping would be a useful tool for conservation biologists monitoring non-uniquely identifiable populations. To fill this gap in methodology, three estimation methods have been put forward. One method, calibrated capture rate indices, links overall capture rate to density (Carbone et al., 2001; Rowcliffe, Field, Turvey \& Carbone, 2008). Another method, the Spatial Capture (SC) model, uses spatial autocorrelation of capture rates to estimate home range center locations (Chandler \& Royle, 2013; Royle, Chandler, Sollmann \& Gardner, 2014). The third method, N-mixture models, uses the difference in capture rates between replicated surveys to estimate the variation in abundance among sites (Royle \& Nichols, 2003). Both the calibrated capture rate indices and the SC model are described and assessed here.

The use of an average capture rate for indexing density was first proposed by Carbone et al. (2001) after an analysis of camera trap studies on tigers (Panthera tigris). Initially, this method was met with great scrutiny from the scientific community, especially in applications with threatened and endangered species. Critics suggested that variations in capture rate due to seasonal variation, camera microhabitat placement, and behavioral avoidance could lead to misleading estimates of density (Janelle, Runge \& MacKenzie, 2001; Foster \& Harmsen, 2012; Sollmann, Mohamed, Sameiima \& Wilting, 
2013c). The calibration of capture rate indices may be impractical or difficult in many situations, making it difficult to produce reliable density estimates. Instead, capture rate indices may be best used for continuous monitoring of individual populations since their application on a broader species level is questionable (Foster \& Harmsen, 2012;

Sollmann et al., 2013c; Villette, 2014). To formalize the capture rate method, the random encounter model (REM) was developed. The REM uses the average capture rate, the distance animals travel in a day and the area visually covered by a camera trap in a twodimensional ideal gas model in order to estimate density (Rowcliffe et al., 2008). Both the REM and the calibrated capture rate work on the same underlying data, average capture rate, but differ in how they use these data to estimate density. Both methods also require additional information for implementation. The calibrated capture rate index requires independent measures of density in order to fit a linear model (Foster \& Harmsen, 2012; Sollmann et al., 2013c), and the REM requires highly detailed day range movements, which can be impractical or impossible to obtain (Rowcliffe, Carbone, Kays, Kranstauber \& Jansen, 2011 \& 2012). Even though these day range movements may be difficult to obtain, encouraging REM field tests using published approximations of day range movements resulted in values similar to independent measures of density (Anile, Ragni, Randi, Mattucci \& Rovero, 2014). Unfortunately, the acquisition of such information for either method may negate the non-invasive benefits of camera trapping.

The SC model uses the spatial autocorrelation of capture rates between closelyspaced traps to estimate density (SC model; Chandler \& Royle, 2013; Royle et al., 2014). The SC model is an extension of Bayesian spatial capture-recapture (SCR) models, which 
use trap location data to estimate density without the need for ad-hoc definitions of the effective trapping area (Efford, 2004; Efford, Borchers \& Byrom, 2009; Royle, Karanth, Gopalaswamy \& Kumar, 2009; O'Brien, 2011). The concept of density as the abundance divided by the area of a trapping grid (i.e., naïve density) only applies when a sample can be made instantaneously, which is rarely, if ever, possible. Animals move through their environment; so any given trapping area will likely experience fluctuating densities, even over very short periods of time. This effect, often called temporary emigration, must be taken into account when estimating animal density. Traditional CR relied on boundary strips to estimate density. Boundary strips are areas added around the outside of a trapping grid that account for individuals whose home ranges partially overlap the trapping grid. The width of the strip was typically estimated as the mean maximum distance moved (MMDM) between traps or one half the MMDM ( $1 / 2$ MMDM) (Dice, 1938; Wilson \& Anderson, 1985; Karanth, 1995; Karanth \& Nichols, 1998; Negroes et al., 2010; Foster \& Harmsen, 2012). Direct comparisons of density estimates from CR with $1 / 2$ MMDM and those from SCR models have shown that both methods perform similarly, though there may be a slight negative bias in SCR estimates (Krebs et al., 2011; Noss et al., 2012; Gerber \& Paramenter, 2015). SCR models address temporary emigration by fully describing a movement and encounter model. The movement model of SCR estimates both home range radius and activity level in conjunction with density. Borrowing from distance sampling approaches, SCR models assume the probability of capture decreases the farther away the trap is placed from a hypothetical home range center (Efford, 2004; Efford et al., 2009; Royle et al., 2009; Royle et al., 2014). This 
description of movement is a form of spatial autocorrelation and assumes an individual is more likely to be caught in the core areas of its range than in the periphery. The SC model makes use of this spatial autocorrelation to estimate individual home range centers from patterns of range use apparent in count-based camera trapping data (although the model has been expanded to presence-absence data by Ramsey, Caley \& Robley, 2015). Estimating density from the pattern of camera capture rates makes the SC model uniquely useful, since it is the only model that can estimate single-site density in non-uniquely identifiable populations from camera data alone.

The SC model and the calibrated capture rate methods represent some of the only choices for non-invasively sampling unmarked populations, but they also have serious drawbacks. This presents both an opportunity and a problem. The opportunity lies in the successful development of one or both of these methods. Either method would provide a tremendously useful tool for those studying non-patterned species, especially species that are difficult to capture or those that could be harmed in the trapping process. Techniques such as live trapping are known to cause considerable stress to the animals captured (Fletcher \& Boonstra, 2006; Delahantey \& Boonstra, 2009). This stress may cause unintended impacts on study populations, which may be a concern with sensitive populations. The problem with these methods lies in the possibility of producing misleading results that are then used to make management decisions for sensitive populations (Janelle et al., 2001; Foster \& Harmsen, 2012; Sollmann et al., 2013c). Because of this conflict, both methods have been the subject of recent studies that examined their validity and attempted to refine them for more robust use. 
The REM has been field tested in several studies, but was only compared against independent estimates of density in a few of them. In studies where the comparison was made, the REM demonstrated reasonable results. The REM produced similar density estimates in studies that compared it against SCR and CR methods (Zero, Sundaresan, O'Brien \& Kinnaird, 2013; Anile et al., 2014). The original Rowcliffe et al. (2008) REM study also produced reasonable density estimates for three of the four species studied. Rovero and Marshall (2008) and Bunty (2015) got disparate density estimates when they compared the REM to distance sampling and fecal accumulation rates. However, Zero et al. (2013) found distance sampling to be less precise than either REM or CR. This suggests that the REM produced the more reliable density estimate in the Rovero and Marshall study. It is difficult to draw conclusions from studies that field tested the REM but did not compare it to independent measures of density. However, the density estimates produced in these studies were consistent with available knowledge on typical population densities for the target species (Manzo, Bartolommei, Rowcliffe \& Cozzolino, 2012; Cole, 2013). The REM has also had several developments including refining of the detection process (Rowcliffe et al., 2011), refining estimates of day ranges (Rowcliffe et al. 2012), and generalizing the model for broader use in methods such as acoustic sampling (Lucas, Moorcraft, Freeman, Rowcliffe \& Jones, 2015).

As the original authors of the SC model have noted (Chandler \& Royle, 2013; Royle et al., 2014), estimates are likely to be imprecise since it estimates several parameters from a limited source of data. To the best of my knowledge, only three field tests of the SC model have been carried out to date (Chandler \& Royle, 2013; Kane, 
Morin \& Kelly, 2015; Ramsey et al., 2015), and only one of these studies (Kane et al., 2015) compared the SC model against an estimate of density where individual identities were known (though only partially, see SMR below). In their study, Kane et al. found that the SC model performed poorly against methods that included individual identification. Ramsey et al. (2015) tested a modified version of the SC model based on presence-absence data and produced imprecise results. Ramsey et al. also noted that presence-absence data reduces the information content available for parameter estimation, which likely made spatial autocorrelation difficult to detect. To improve precision and increase the usefulness of the model, Chandler and Royle (2013) showed how the SC model could be used when a portion of the population's identities are known. This is commonly referred to as a SMR, or spatial mark-resight (Royle et al., 2014). SMR has been quickly implemented by researchers with promising results, though the precision of its estimates were still relatively low compared to models that include perfect individual identification (Kane et al., 2015; Rutledge, Sollmann, Washburn, Moorman \& De Perno, 2015; Sollmann et al, 2013a \& 2013b; Rich et al., 2014). Thus, the SC model still requires testing against robust measures of density. Specifically, more information is needed on trap effort and the amount of spatial autocorrelation required to produce viable density estimates (Royle et al. 2014).

Because all camera trapping methods depend in some way on capture rates, it is worthwhile to assess variation in detection rates within camera trap models. Studies often utilize different cameras models throughout a grid, which can lead to unknown differences in detectability between cameras and bias estimates of density. Models such 
as the REM have guidelines for assessing this variation (Rowcliffe et al., 2008), which have been implemented in several studies (Manzo et al., 2012; Anile et al., 2014; Bunty, 2015). This issue may not be as severe for the SC model as inference is made on relative capture rates between traps. In this case, homogenous failure rates would only indirectly affect density estimates through lower apparent effort. However, heterogeneous capture rates within a specific camera trap model may cause problems using the SC model. Unfortunately, several studies have found considerable variation in detection rates both between and within camera models in field studies (Damm, Grand \& Barnett, 2010; Hughson, Darby \& Dungan, 2010) and controlled laboratory settings (Swann, Hass, Dalton \& Wolf, 2004). Field studies comparing camera detection rates have examined the relative capture success of cameras placed side-by-side, but this method may not account for instances when all cameras fail to capture an event. Likewise, laboratory tests of camera traps may fail to accurately represent true field conditions. Thus, methods that can accurately estimate camera success rate in field conditions are of great value to researchers.

To test the SC model and calibrated captures rates against independent estimates of density, a simultaneous live trapping and camera trapping study was conducted on a population of kangaroo rats (Dipodomys spp.). Given the number of underlying movement assumptions in these models, kangaroo rats are an excellent focal group since they are easy to live capture and a wealth of telemetry information exists describing their movement habits (Schroeder, 1979; Behrends, \& Wilson, 1986; Perri \& Randall, 1999; Shier \& Randall, 2004; Cooper \& Randall, 2007; Meshriy, Randall \& Parra, 2011). In 
California, kangaroo rats are a species of ecological concern since over half the known species of kangaroo rats occur in the state, five of which are endemic. Many kangaroo rat species have limited ranges and often occur on highly sought after valley floor land. These factors place kangaroo rats at higher risk of extirpation due to encroaching human development (Goldingay, Kelly \& Williams, 1997). Species such as the short-nosed, Santa Cruz, and San Bernardino kangaroo rats (D. n. brevinasus, D. venustus venustus and D. merriami parvus, respectively) have all been listed as Species of Special Concern by the California Department of Fish and Wildlife (Brylski, Collins, Pierson, Rainey \& Kucera, 1998), and the giant, Fresno, Tipton, and Stephen's kangaroo rats (D. ingens, $D$. nitratoides exilis, D. $n$. nitratoides, and D. stephensi, respectively) are all listed as endangered by the U.S. Fish and Wildlife Service (D. n. exilis - Potter, 1985; D. ingens Smith, 1986; D. n. nitratoides - Recce, 1988; D. stephensi - Recce, 1988). Even worse, the Morro bay kangaroo rat (D. heermanni morroensis) is already thought to be extinct in the wild (Stewart \& Roest, 1960; Congdon \& Roest, 1975), and the Berkeley and lesser California kangaroo rats have not been observed in decades (Goldingay et al., 1997). In order to better protect and monitor these crucial populations, safer and more efficient monitoring methods need to be developed and implemented so that land managers can make informed decisions for conserving these unique populations.

In this study, I tested the abilities of the SC model and calibrated capture rate indices to estimate small mammal population density. I assessed the accuracy of their density estimates by comparing them against live trapping estimates where individual identity was included. Furthermore, I studied the focal population over the course of ten 
sessions to examine how both methods respond to changes in population size. For the SC model, I attempted to determine the camera-to-abundance ratio needed in order to sufficiently estimate population parameters. I then provide suggestions on how to refine density estimates using the SC model. Finally, I devised and implemented a new method for detecting missed capture events from the camera trap data directly. 


\section{Methods}

\section{Project Site}

The study was located in Pinnacles National Park (36.497 $\left.{ }^{\circ},-121.152^{\circ}\right), 30$ miles south of Hollister, California (Figure 1). Within the park, the study site was at a location referred to as Sphinx Canyon by park officials. Sphinx is a side branch of McCabe Canyon located near the eastern border of the park. Much of McCabe Canyon is a designated wilderness area with restricted access to most park visitors, which allowed for low levels of human disturbance. Three habitat types made up the majority of the project site: canyon floor, southeast-facing canyon slopes and northwest-facing canyon slopes.

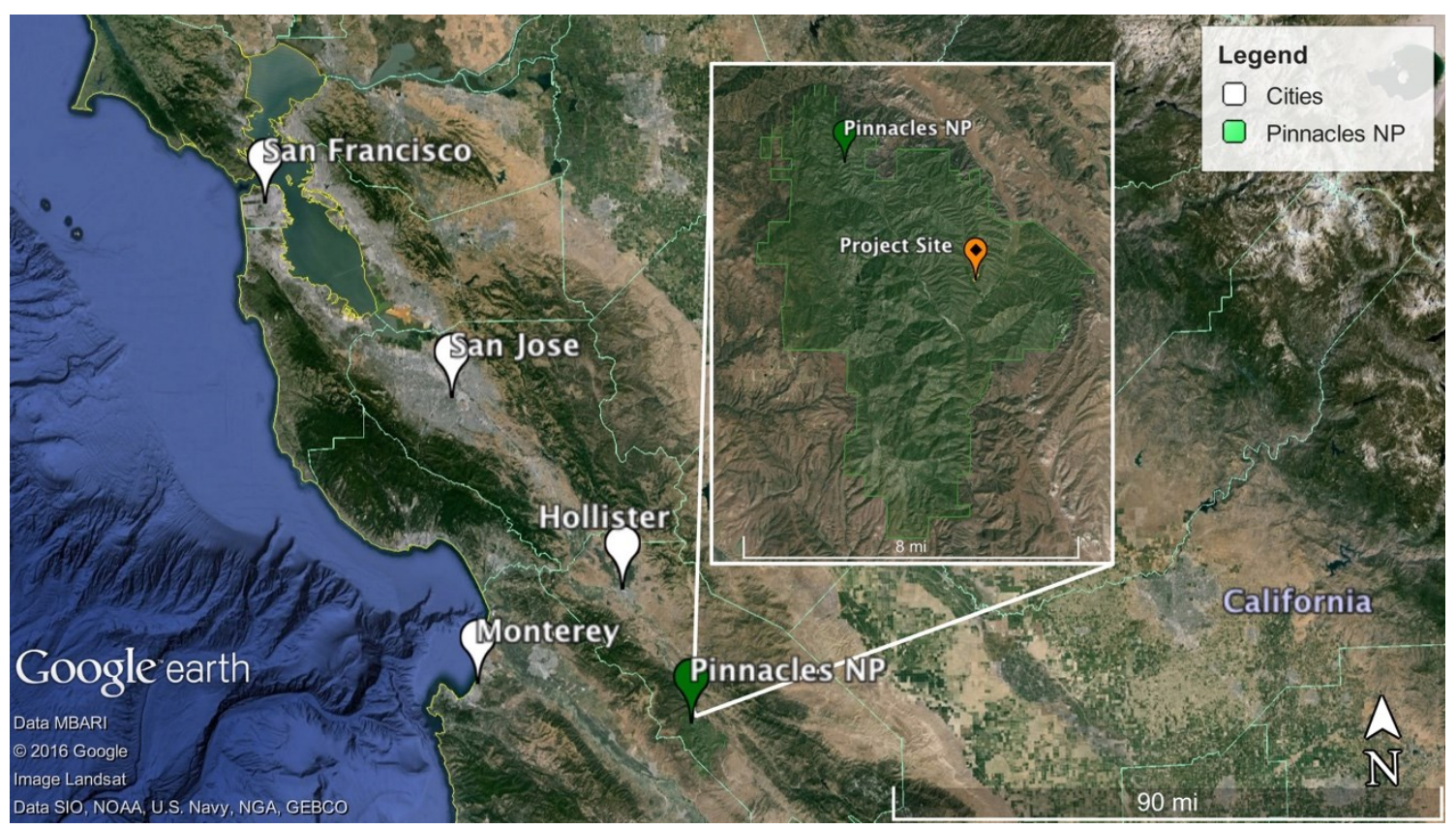

Figure 1. Map showing the location Pinnacles National Park in California. Inset map shows the Pinnacles park boundary and the location of the project site within the park. 
The canyon floor was a sandy wash dominated by California buckwheat (Eriogonum fasciculatum), interspersed with annuals and shrub species such as golden aster (Heterotheca sessiliflora), elegant buckwheat (Eriogonum elegans), and red-stemmed filaree (Erodium cicutarium). The southeast-facing canyon slopes were composed of a mix of chamise (Adenostoma fasciculatum) and California buckwheat that were separated by stretches of sparse ground cover. The ground cover included species such as coffee fern (Pellaea andromedifolia) and chia sage (Salvia columbaria). The northwest-facing canyon slopes were an association of chamise and wedge-leaf ceanothus (Ceanothus cuneatus), with sections of relatively open areas covered in annuals and California buckwheat. An understory of forbs was persistent on the northwest canyon slopes for several months, which included species such as miner's lettuce (Claytonia perfoliata). Pinnacles National Park has an arid climate and a mean annual rainfall of $40 \mathrm{~cm}$ that occurs during the cool, winter months. Winter daytime temperatures of $10-16^{\circ} \mathrm{C}$ are typical, and night time temperatures regularly fall below $-5^{\circ} \mathrm{C}$. Little, if any, rain falls during the summer, and daytime summer temperatures regularly exceed $38^{\circ} \mathrm{C}$. Late winter and early spring rains herald an impressive spring bloom in McCabe Canyon. Vegetation heights changed dramatically between subsequent visits spaced two to three weeks apart. Annuals in the lower reaches of Sphinx Canyon appeared to grow 20-40 cm, occasionally interfering with camera sensors.

\section{Study Species}

This study focused on a population of kangaroo rats (Dipodomys spp.). Kangaroo rats are nocturnal rodents that inhabit a variety of arid climates throughout the United 
States. Some species, such as Merriam's kangaroo rat (D. merriami), have been well studied for their ability to survive on dry diets (Schmidt-Nielsen, Schmidt-Nielsen, Brokaw \& Schneiderman, 1948; Schmidt-Nielsen \& Schmidt-Nielsen, 1950; Tracy \& Walsberg, 2001a, 2001b \& 2002). Kangaroo rats are primarily granivorous but are also known to eat green vegetation and insects (Hawbecker, 1940; Tappe, 1941; Tracy and Walsberg, 2001a \& 2001b).

The species captured most frequently during this study was Heermann's kangaroo rat (D. heermanni). Heermann's kangaroo rat is a medium-sized kangaroo rat; specimens examined by Grinnell (1922) from the Pinnacles locality weighed between 61.2-88.4 g with an average mass of $68.1 \mathrm{~g}$. Heermann's kangaroo rats are found in a range of habitats including coastal dunes, sparsely vegetated knolls, sandy valley floors, and finersoiled grasslands (Kelt, 1988). Studies of Heermann's kangaroo rat seed casings and cheek pouch contents have shown that they eat large amounts of non-native grasses and forbs such as Bromus, Erodium, and Avena species (Tappe, 1941; Fitch, 1948). Shier and Randall (2004) studied the movement and territoriality behaviors of coastal Heermann's Kangaroo rats and found that home ranges were sexually dimorphic in size with strong intrasexual territoriality. Their findings suggested that females maintained a minimum home range for collecting resources, while males maximized their home range size to gain access to females.

Several narrow-faced kangaroo rats (D. venustus) were also captured during the course of this study. The narrow-faced kangaroo rat is typically larger than Heermann's kangaroo rat, and specimens of the closest geographic subspecies (formerly a distinct 
species; see Best, Chesser, McCullough \& Baumgardner, 1996) D. v. elephantinus and D. v. santiluciae examined by Grinnell (1922) ranged from 79.4-90.7 $\mathrm{g}$ with averages around $85 \mathrm{~g}$. Comparatively little is known about this species, and the only published studies examining the natural history of this species date to the 1940s and 1970s. Narrowfaced kangaroo rats tend to live in more mesic habitats than most kangaroo rat species and are thought to require some free water in order to survive (Church, 1969). Bradford (1976) found that narrow-faced kangaroo rats were most frequently captured towards the interior of chamise stands, whereas most other species captured were located along the periphery. Hawbecker (1940) reported large quantities of telegraph weed (Heterotheca grandiflora) seeds stored in narrow-faced kangaroo rat burrows and suggested that this was the preferred food source.

\section{Live Trapping Protocol}

Twelve 3-night live trapping sessions were conducted from April 2014 to May 2015 (Table 1). Out of the 12 separate live trapping sessions conducted, two live trapping sessions were dropped from the final analysis: one from September 2014 and one from May 2015. In September 2014, camera trap records revealed that the live trapping grid was raided by several raccoons (Procyon lotors). These raccoons disturbed up to $50 \%$ of the trap grid each night and may have preyed on kangaroo rats, though no direct evidence exists. There were additional instances where individual traps were disturbed, possibly by raccoons or some other large mammal, but not significantly again until May 2015. On the final night of live trapping in May 2015, large portions of the trap line were disturbed again by raccoons. Thus, this trapping session was also excluded from the analysis. 
During each live trapping session, 120 long-type Sherman live traps were placed in a grid throughout the canyon floor, except in session S1 when 140 traps were used. Traps were placed approximately $10 \mathrm{~m}$ apart from one another in a grid that extended up the valley floor. A periphery of traps was also placed on the canyon slopes to monitor potential changes in species composition along the edges of the canyon floor. Traps were placed in approximately the same locations during each session. However, to account for subtle changes in trap location in SCR analysis, live trap GPS locations were recorded for all sessions (see Figure 2 for approximate locations and Appendix A1 for exact locations per session). All GPS coordinates $( \pm 1.0 \mathrm{~m})$ taken during this study were recorded using a Trimble Geo-XH, and a minimum of 20 vertex readings were recorded per trap.

Traps were opened at sunset and checked each morning before the sun reached the canyon floor. All traps were baited using crimped oats to provide nutrition and stuffed with polypropylene for insulation. After several mortalities occurred on cold nights, the baiting and insulation protocols were modified. Initially the oats were loosely scattered in the backs of traps by volunteers. This created a potential problem wherein oats could become stuck under the treadle and bridge of the trap, out of reach to the animal. To insure accessibility, the oats were stuffed into the center of the polypropylene ball. This new packing method coincidentally allowed for increased amounts of polypropylene to be inserted in the traps, further increasing the level of safety. No further mortalities occurred due to cold stress once this procedure was implemented.

To track individual identities, all captured animals were ear tagged, except for species or age classes with ears were too small to hold tags. This was the case for western 
harvest mice (Reithrodontomys megalotis; hereafter, harvest mice), California pocket mice (Chaetodipus californicus; hereafter, pocket mice), and juvenile Peromyscus spp. Instead of receiving ear tags, the venters of these individuals were marked with a permanent marker in order to provide data for mark-recapture analysis. For every animal captured, trap number, mass, age class, reproductive status, and sex were determined. Morphometric measurements were collected for certain species to aid in species identification. Measurements were recorded for several captures to insure accuracy. Ear and hindfoot measurements were taken in millimeters for both pinyon mice (P. truei) and brush mice $(P$. boylii). Individuals with a hindfoot length greater than or equal to their ear length were determined to be brush mice (Kays \& Wilson, 2009). Kangaroo rat ear lengths were also measured. Individuals were determined to be narrow-faced kangaroo rats if they had an ear length of $18 \mathrm{~mm}$ or greater, an overall darker dorsal coloring, and a greater mass. Individuals were determined to be Heermann's kangaroo rats if they had an ear length less than $17 \mathrm{~mm}$, a blonder coat coloration, and a more distinct "moustache" (Kays \& Wilson, 2009). Live trapping data were recorded in the field and later transcribed onto a spreadsheet. Once all raw data were recorded, a copy was made, and individual tag records were scrutinized for potential errors and to confirm species identifications. All tag records with ambiguous species identities were compared across captures and morphometric measurements so that a single species identification could be made for the record. Individuals that escaped before tags were read were excluded from analysis. 
All live trapping was conducted in accordance with the guidelines set forth by San José State University's Institutional Animal Care and Use Committee (Protocol \#995), Pinnacles National Park, and those suggested by the American Society of Mammalogists (Gannon \& Sikes, 2011).

\section{Camera Trapping Protocol}

Camera trapping was carried out from April 2014 through May 2015. The study began with 20 Reconyx HC 450 Hyperfire cameras, but several units malfunctioned and only 16 were operational by May 2015 (see Table 3 for camera outage data). Cameras were placed in different gridded patterns throughout the live trap study area (Figure 2).
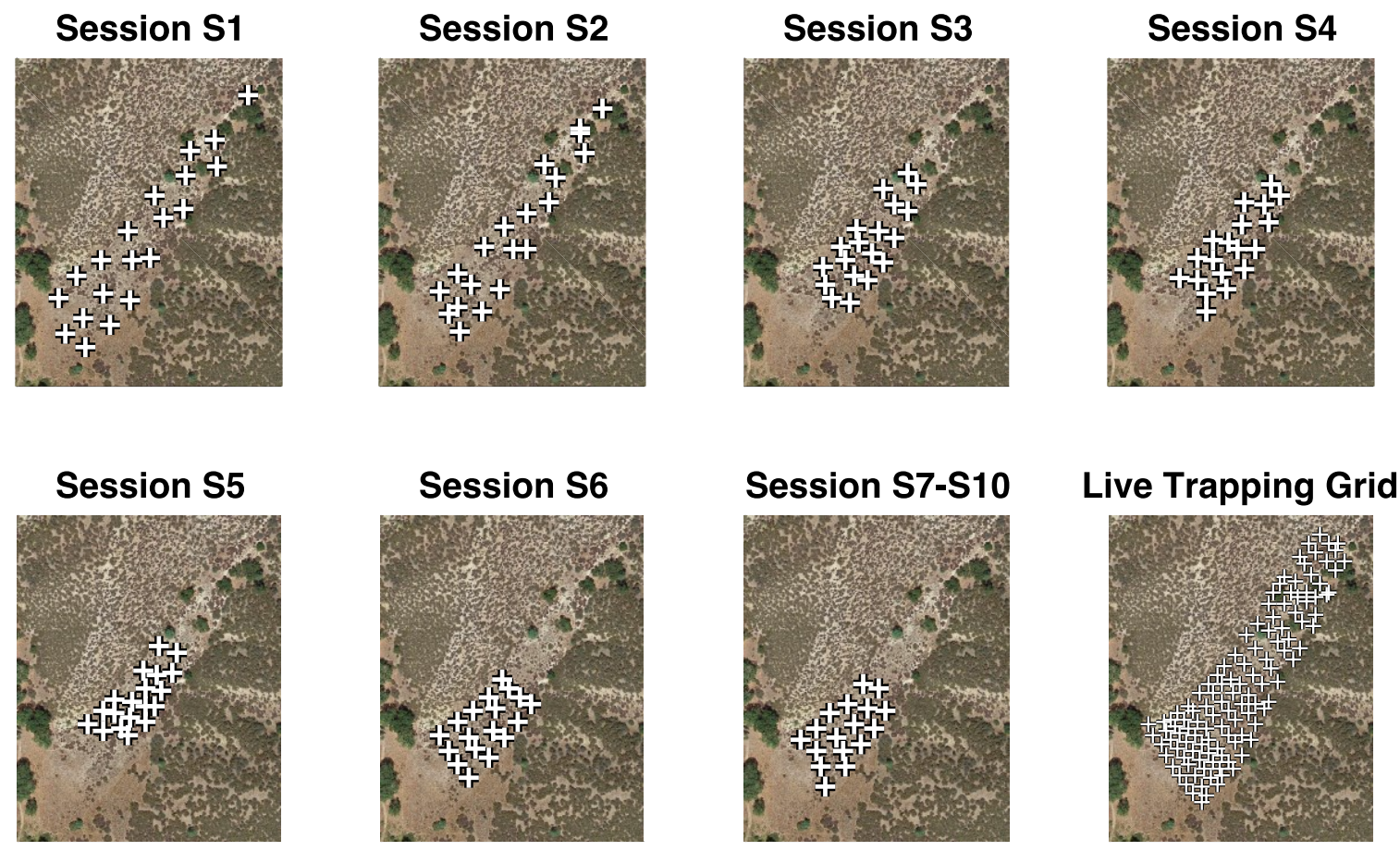

Figure 2. Maps showing the layout of the camera grid for all seven placements. The average layout of the live trapping grid is also show in the bottom right for reference. 
The area covered by the camera trapping array was reduced over time in order to examine changes in SC model performance when the camera-to-abundance ratio and amount of spatial autocorrelation were increased. GPS coordinates were recorded for each individual camera placement. Cameras were mounted onto custom-built stands and covered in camouflage fabric to reduce the risk of theft. Cameras were mounted at a height of approximately $1 \mathrm{~m}$ and were pointed downward at a $45^{\circ}$ angle. This optimized the cameras for capturing kangaroo rats and allowed species of all other sizes to be captured.

Cameras were operated jointly on a passive infrared (IR) motion sensitive trigger and on a 5 min interval (Cutler \& Swann, 1999). Triggered events recorded 10 images for every detection and were in operation continuously. Images captured on the 5 min interval were only collected at night to reduce the usage of batteries and memory cards. If an interval event occurred during a trigger event, the interval picture was captured between the triggered images. The benefit of the 5 min interval was two-fold: 1 ) it allowed for additional captures of animals that did not trigger the motion system, and 2) it provided information on the missed capture rate. When animals passed by the field of view but were not detected by the motion detector, a trail visible to the reviewer was typically left in the soil. Missed image rates were determined per session. However, miss rates were strongly influenced by the number of ectothermic animals present. Since the camera sensors were not built to detect these species, ectothermic misses were not counted as true misses. The number of endothermic capture events missed, $\widehat{M}_{e n}$, was 
estimated as $\widehat{M}_{e n}=M_{t} \cdot I_{e n} /\left(I_{e n}+I_{e c}\right)$. Here, $I_{e n}$ and $I_{e c}$ are the number of endothermic and ectothermic interval images, respectively, and $M_{t}$ is the total number of missed events.

The endothermic miss percentage was then calculated as $\widehat{M}_{e n} /\left(T+I_{e n}+\widehat{M}_{e n}\right)$. Here, $T$ is the number of triggered captures, which are assumed to come from endothermic species. To assess the effect of ambient temperature on miss rate, the estimated endotherm miss percentage was compared to average nighttime temperatures per session in a linear regression. Average nighttime temperatures were calculated from temperatures recorded by the cameras on interval images. Additionally, the heterogeneity of individual camera's detection rates was assessed by examining the effective miss rate per camera, per session. Since interval images were technically missed by the motion sensor, the effective miss rate was calculated as $\left(\widehat{M}_{e n}+I_{e n}\right) /\left(T+I_{e n}+\widehat{M}_{e n}\right)$. Some cameras were dropped from the analysis to maintain sufficient statistical power. As such, effective miss rates were calculated for the eight cameras with the most days active over all 10 sessions. A Kruskall-Wallis test was then performed to assess whether or not there was significant variability in detection between cameras.

Operating the cameras jointly in triggered and interval modes resulted in a large volume of image data. In order to analyze this large volume of data, images were processed using purpose-built routines in MATLAB (The Mathworks, ver 2015b, Natick, MA) and EXIFTOOL (Harvey, 2015). The routine recorded capture times, temperatures, moonphase, trigger type, sequence image, and image mode (IR or color) directly from the image metadata and re-wrote the information into a field that could be easily viewed in 
standard photo management software. All day-time images were excluded from review using this process, though day-time images were sporadically checked for kangaroo rats. Only one daytime capture was discovered and was determined to be from a live trapping event. The same MATLAB routine also processed images through a motion detection algorithm. Two algorithms were used over the course of the study. The initial system used a simple difference between images to detect movement. This initial system missed $10-15 \%$ of small movements and required additional time to correct mistakes. This motivated the development of a second algorithm that was able to achieve much more accurate results. The second algorithm used a more complex detection system that could reliably exclude up to $25-50 \%$ of images where no motion occurred and also detect small disturbances in the soil for the miss analysis. The second algorithm allowed for substantially decreased image review times. Regardless of the algorithm used, tracks left behind by animals passing through the frame were sufficient to catch any animals missed by the motion detection algorithm. Thus, the review process was considered to be robust throughout the project.

Images were managed in Phase One Media Pro (Phase One A/S, 2012; hereafter, "Media Pro"), which was chosen for its ability to quickly review images and manage metadata. All images taken were loaded into Media Pro catalogs and sorted in review sets based on the motion detection results. Images marked with potential motion were reviewed manually. Since several images were recorded for each encounter, a single image was chosen to serve as the data point. Species data were attached to all images containing animals. Species-level identifications for Dipodomys and Peromyscus captures 
were not possible; instead, these two groups were identified by their genus. Missed captures were recorded as two images from which disturbances in the soil could be analyzed.

Encounters were defined as an animal entering the field of view and not leaving for more than $15 \mathrm{~s}$. If an animal left the frame of view for more than $15 \mathrm{~s}$, the set of images was treated as two events. Whether or not an animal had left the frame between images greater than $15 \mathrm{~s}$ apart was determined visually by using best judgment of disturbance patterns in the soil. This method produced capture data with a hit window of $15 \mathrm{~s}$, which resulted in significant overdispersion of the captures rates. To reduce the effects of overdispersion on model fit, capture histories with hit windows of $15 \mathrm{~s}, 15 \mathrm{~min}$, $30 \mathrm{~min}, 1 \mathrm{~h}, 2 \mathrm{~h}, 4 \mathrm{~h}$, and $16 \mathrm{~h}$ were assembled. For each camera on each session that had at least one capture, a variance-to-mean ratio was calculated using the daily capture counts. Then, the mean variance-to-mean ratio over all cameras and sessions was calculated for each of the seven hit windows. The hit window that produced the mean variance-to-mean ratio closest to one was chosen for analysis.

\section{Analysis of the Calibrated Capture Rate Index and Spatial Capture Model}

To test the effectiveness of the calibrated capture rate method and compare it against density estimates from the SC model, multiple linear regressions were performed in $\mathrm{R}$ ( $\mathrm{R}$ Core Team, 2015). One regression compared the density estimates from the bestfitting SC model against those from live trapping. Another compared both the capture rate and the miss rate to live trapping density. Finally, a regression comparing only capture rate and live trapping density was performed to assess the change in $\mathrm{R}^{2}$ when the 
missed capture information was dropped from the analysis. Although the size and shape of the camera grid changed over the course of this study and the live trapping grid did not, density estimates were assumed to remain stable over these differences in sample scale. Also, since the live trapping dataset sampled the most unique individuals, it is thought to have the best estimates of density to compare others against.

\section{Spatial Capture-Recapture Analysis}

The camera and live trapping data were combined in multiple ways to produce density estimates. First, the 10 sessions of camera data were analyzed. Each session lasted 20-28 days, and a corresponding live trapping session typically occurred in the middle of the camera session. These camera data are referred to as the "CT" dataset and were fitted to the $\mathrm{SC}$ model. The corresponding live trapping data are referred to as the "LT" dataset and were fitted to a multinomial SCR model for direct comparison against the SC model. Subsequently, the camera data were arranged into five additional sessions so that 9-12 days of camera data from two separate camera placements were combined. These data are referred to as the "CTMix" dataset and were also evaluated under the SC model. The CTMix session dates overlap with many of the dates from the CT dataset and the corresponding LT session typically occurred directly after the CTMix session. This combined dataset resulted in capture histories with twice the number of camera locations but half the number of sampling occasions. The use of this data structure was motivated by the suggestions of Foster and Harmsen (2012) and Rowcliffe et al. (2008) on how to best estimate density when camera availability is limited. Finally, both the CT and CTMix datasets were combined with the LT dataset to form the "CT-LT" and "CTMx- 
LT" datasets. These datasets used a combined model that evaluated the information from both trapping datasets simultaneously. This was done in order to test the SC model's ability to estimate range size from the camera trapping data given abundance information from the live trapping data. Also, parameter estimates from the CT-LT and CTMx-LT datasets provided the most direct information with which to compare the $\mathrm{CT}$ and CTMix datasets against. Dates for all of the datasets can be found in Table 1.

Bayesian implementations of SCR models have been described in many publications (Efford et al., 2009; Royle \& Nichols, 2003; Royle et al., 2009; Borchers, 2012) and in a detailed text (Royle et al., 2014). Here, description of SCR models is limited to the basic structure and to any modifications implemented during this study. An in-depth description of the models used in this study can be found in Appendix B.

Table 1. List of session number and trapping dates from all datasets: LT, CT, CT-LT, CTMix and CTMx-LT. All CT dates that overlap LT dates were excluded from analysis and the CT day count.

\begin{tabular}{|c|c|c|c|c|c|c|c|}
\hline Session & Datasets & LT Date Start & LT Date End & $\begin{array}{c}\text { LT } \\
\text { Days }\end{array}$ & CT Date Start & CT Date End & $\begin{array}{c}\text { CT } \\
\text { Days }\end{array}$ \\
\hline S1 & LT, CT \& CT-LT & 5 Apr, 2014 & 8 Apr, 2014 & 3 & 31 Mar, 2014 & 30 Apr, 2014 & 27 \\
S2 & LT, CT \& CT-LT & 10 May, 2014 & 13 May, 2014 & 3 & 2 May, 2014 & 2 Jun, 2014 & 28 \\
S3 & LT, CT \& CT-LT & 18 Oct, 2014 & 21 Oct, 2014 & 3 & 18 Sep, 2014 & 17 Oct, 2014 & 29 \\
S4 & LT, CT \& CT-LT & 8 Nov, 2014 & 11 Nov, 2014 & 3 & 20 Oct, 2014 & 22 Nov, 2014 & 30 \\
S5 & LT, CT \& CT-LT & 6 Dec, 2014 & 9 Dec, 2014 & 3 & 23 Nov, 2014 & 23 Dec, 2014 & 27 \\
S6 & LT, CT \& CT-LT & 3 Jan, 2015 & 6 Jan, 2015 & 3 & 24 Dec, 2014 & 16 Jan, 2015 & 20 \\
S7 & LT, CT \& CT-LT & 31 Jan, 2015 & 3 Feb, 2015 & 3 & 17 Jan, 2015 & 13 Feb, 2015 & 24 \\
S8 & LT, CT \& CT-LT & 28 Feb, 2015 & 3 Mar, 2015 & 3 & 14 Feb, 2015 & 14 Mar, 2015 & 25 \\
S9 & LT, CT \& CT-LT & 28 Mar, 2015 & 31 Mar, 2015 & 3 & 15 Mar, 2015 & 11 Apr, 2015 & 24 \\
S10 & LT, CT \& CT-LT & 25 Apr, 2015 & 28 Apr, 2015 & 3 & 12 Apr, 2015 & 10 May, 2015 & 25 \\
S1+S2 & CTMix \& CTMx-LT & 10 May, 2014 & 13 May, 2014 & 3 & 21 Apr, 2014 & 9 May, 2014 & 15 \\
S3+S4 & CTMix \& CTMx-LT & 18 Oct, 2014 & 21 Oct, 2014 & 3 & 9 Oct, 2014 & 30 Oct, 2014 & 18 \\
S4+S5 & CTMix \& CTMx-LT & 6 Dec, 2014 & 9 Dec, 2014 & 3 & 10 Nov, 2014 & 5 Dec, 2014 & 25 \\
S5+S6 & CTMix \& CTMx-LT & 3 Jan, 2015 & 6 Jan, 2015 & 3 & 15 Dec, 2014 & 2 Jan, 2015 & 18 \\
S6+S7 & CTMix \& CTMx-LT & 31 Jan, 2015 & 3 Feb, 2015 & 3 & 5 Jan, 2015 & 30 Jan, 2015 & 25 \\
\hline
\end{tabular}


SCR models estimate animal density by explicitly stating an animal movement model as well as an observation model. Animal movement is described in SCR models as a function of distance from a hypothetical home range center, $\boldsymbol{s}_{i}$. The concept of a home range center works well in the case of kangaroo rats, since they often occupy a central burrow from which they forage about. However, home range centers do not need to be a central nest or burrow; instead, they represent the geometric center of the area an animal moves over during a trapping occasion (Royle et al., 2014). Home range centers are realizations of a point process and typically have a uniform distribution across a state space, $S$, such that $\boldsymbol{s}_{i} \sim$ Uniform $(S)$. In general, so long as $S$ is sufficiently large, density results are thought to be unbiased (Royle et al., 2014).

The probability of encountering a specific individual at a given trap is described by a detection function that relates $\boldsymbol{s}_{i}$ to each of the $J$ trap locations, $\boldsymbol{x}_{j}$. Any monotonically decreasing function may serve as a detection function, and several variations have been put forth in the SCR literature (Efford, 2004; Efford et al., 2009; Royle et al., 2014). For this study, a new detection function (Figure 3) was developed to unify the previously described detection functions. This function also placed the emphasis on the biologically relevant features of the function for easier interpretation by readers. The function is given as:

$$
d\left(x_{j}, s_{i}\right)=\exp \left(z \frac{\left\|x_{j}-s_{i}\right\|^{a}}{\sigma^{a}}\right)
$$

Here, $\sigma$ is analogous to a home range radius and is explicitly defined as the distance at which the detection function reaches a sufficiently low point controlled by $z=\log \left(p_{c}\right)$. 
Where $p_{c}$ was the height of the detection function at a distance of $\sigma$ from $\boldsymbol{s}_{i}$ when the encounter rate parameter (e.g., $\lambda_{0}$ or $e^{\alpha_{0}}$ ) equals one. With other detection functions the interpretation of home range radius changes depending on the function used. For example, the half normal detection function has an estimated home range radius of $2.45 \sigma$ (Ramsey et al., 2015), which is not straight-forward to interpret. Here, $p_{c}$ was set at 0.01 .

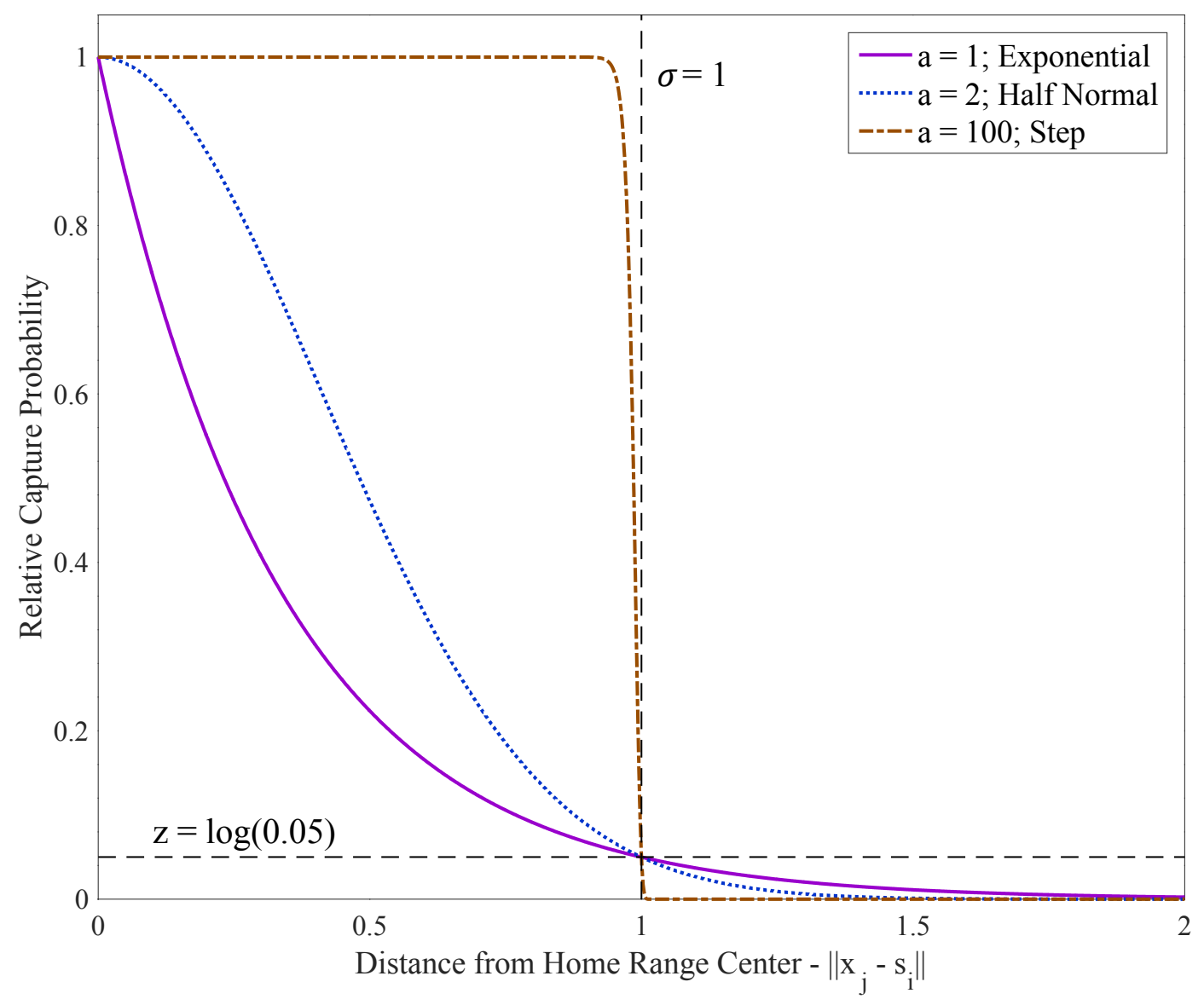

Figure 3. Graph showing the detection function, $d\left(\boldsymbol{x}_{\boldsymbol{j}}, \boldsymbol{s}_{i}\right)$, set to different values of $a$. The intersection of all three variations at $z$ and $\sigma$ shows how the biologically relevant factor, $\sigma$, has the same interpretation no matter the shape. 
Parameter $a$ controlled the shape of the function. When $a=1$ the function's shape resembled the exponential detection function; when $a=2$ it resembled the half normal; if $a$ was set high (e.g., 100), it resembled the step function. All datasets were fitted using approximations of the Exponential ("EP;" $a=1$ ) and Half Normal ("HN;" $a=2$ ) detection functions.

Abundance, $N$, was estimated through Parameter Expanded Data Augmentation (hereafter, "data augmentation"), which allowed for a model formulation that was conditional on $N$ (Royle et al., 2009; Chandler \& Royle, 2013; Royle et al., 2014). Under data augmentation $N$ was estimated using $w_{i}$, which was a set of $M$ zero or one indicator variables distributed as $w_{i} \sim \operatorname{Bernoulli}(\psi)$. Using data augmentation $N=\sum w_{i}$ and $\psi \approx E[N] / M$. Data augmentation allows potentially unobserved but present individuals in the population (e.g., sampling zeros) to be estimated using Markov chain Monte Carlo (MCMC) methods (Royle, Dorazio \& Link, 2007; Royle et al. 2014).

\section{Analysis of live trapping data using the multinomial observation model.}

Considering CR's roots in live trapping, the issue of choosing an appropriate observation model for single catch traps has proven to be surprisingly difficult (Otis, Burnham, White \& Anderson, 1978; White, 1982; Efford, 2004; Balderama, Gardner \& Reich, 2012; Royle et al., 2014). As traps fill up throughout the night, the probability of individuals not yet trapped encountering an available trap decreases. This probability could be factored into the resulting analysis if the order of trapping was known, but this order is rarely, if ever, known (Efford, 2004; Balderama et al., 2012). Simulation studies have suggested that the multinomial observation model provides reasonable estimates when trap 
saturation is low (Efford et al, 2009; Balderama et al. 2012). This formulation falsely assumes that every individual present in the population can be captured at any of the traps at any point in a trapping night (Efford, 2004; Efford, 2009; Royle et al., 2009, 2014). In these situations, the trapping rate parameter, $\alpha_{0}$, will be increasingly underestimated as trap saturation increases (Efford et al., 2009). Since trap saturation throughout this study was typically low $(<20 \%)$, the multinomial SCR model was implemented, and the violations of its assumptions were assumed to have minimal consequences.

The multinomial observation model described in Royle et al. (2014) was used here. In this model a matrix of capture data, $y_{i j}$, described the number of times individual $i$ was captured at trap $j$ during $K$ trapping occasions. Thus, $y_{i j} \sim \operatorname{Multinomial}\left(K, \pi_{i j}\right)$ where $\pi_{i j}$ was a matrix describing the probability of individual $i$ being caught at trap $j$, and instances where $w_{i}=0$ were ignored.

$$
\pi_{i j}=\frac{\exp \left(\alpha_{0}\right) d\left(x_{j}, s_{i}\right)}{1+\sum_{j=1}^{J} \exp \left(\alpha_{0}\right) d\left(x_{j}, s_{i}\right)}
$$

An additional element was added to the position $\pi_{i J+1}$ to account for the probability of an individual not being captured on a given trapping night and was calculated as:

$$
\pi_{i J+1}=\frac{1}{1+\sum_{j=1}^{J} \exp \left(\alpha_{0}\right) d\left(x_{j}, s_{i}\right)}
$$

Here, $\alpha_{0}$ was the encounter rate parameter, and as it increased, the probability of not being captured at any trap decreased.

Analysis of camera trapping data using the spatial capture model. The SC model was implemented largely based on the R routine written by Chandler and Royle (2013) and adapted in MATLAB. The SC model utilized the Poisson observation model, 
which is typical for modeling the capture process of camera traps (Efford et al., 2009; Royle et al., 2009; Royle et al., 2014). Under the SC model, the capture data were a set of $y_{t j}$ image counts that corresponded to the number of captures at trap $j$ on day $t$. These data were distributed as $y_{t j} \sim$ Poisson $\left(\lambda_{t j}\right)$, where $\lambda_{t j}=\lambda_{0} c_{t j} \sum_{i=1}^{M}\left[w_{i} d\left(x_{j}, s_{i}\right)\right]$ and $\lambda_{0}$ described the maximum encounter rate if a camera trap was located directly on an animal's home range center. The variable $c_{t j}$ was set to one if a camera trap was active on a given day and set to zero otherwise. This was added to Chandler and Royle's (2013) original implementation to account for periodic camera outages due to malfunctions.

Following Chandler and Royle's (2013) suggestions for improving posterior precision, the camera trap data under the SC model were fitted with both an uninformative uniform prior on $\sigma$ as well as with a $\operatorname{Gamma}(23,1)$ prior. The informative prior's shape parameters were chosen from published descriptions of $D$. heermanni home range sizes from both radio tracking (Shier \& Randall, 2004) and live trapping (Fitch, 1948). The prior on $\sigma$ was also chosen based on initial estimates of home range size from live trapping data gathered during this study. Analyses with the prior on $\sigma$ (referred to as "SP" sessions) were performed on both the CT and the CTMix datasets using both the $\mathrm{HN}$ and EP detection functions. This resulted in four sets of posterior estimates for each session in the CT and CTMix datasets.

Joint analysis of camera and live trapping data. To simultaneously fit the camera trap and the live trap data, certain parameters were selected to be fitted against the dataset that was thought to provide the best estimates. For estimations of home range size, $\sigma$, and camera capture rate, $\lambda_{0}$, the likelihood from the camera trapping data was 
used. The live trapping likelihood was then used to fit parameter estimates of live trapping capture rate, $\alpha_{0}$, and abundance, $N$ and $w_{i}$. Finally, the product of both full conditional likelihoods was used to fit estimates of individual home range centers, $\boldsymbol{s}_{i}$.

Since the live trapping grid was typically larger than the camera trapping grid, portions of the live trapping data needed to be excluded in order to combine the datasets. All live trap capture histories that fell outside of the $30 \mathrm{~m}$ buffer placed around the camera trap locations were excluded from analysis in the CT-LT and CTMx-LT datasets (see CT-LT and CTMx-LT capture histories in Appendix A2 and A3).

Assessing model fit. Following the recommendations in Royle et al. (2014), Bayesian p-values were used to assess differences in model fit between different model implementations. Bayesian p-values were generated by comparing the observed data to a "perfect" dataset generated by the model. To do this, a random dataset is simulated during each posterior sample to serve as the perfect dataset. Both the simulated perfect dataset and the observed dataset are compared against the current expected values (i.e., the current posterior sample) using the equation:

$$
T=\sum_{j}^{J}\left(\sqrt{Y_{j}}-\sqrt{\mathrm{E}\left(Y_{j}\right)}\right)^{2}
$$

Where $Y_{j}=\sum_{i}^{M} y_{i j}$ and $E\left(Y_{j}\right)=K \sum_{i}^{M}\left[\pi_{i j} w_{i}\right]$ for the live trapping data, and $Y_{j}=\sum_{t}^{T} y_{t j}$ and $E\left(Y_{j}\right)=\sum_{t}^{T} \lambda_{t j}$ for the camera trapping data. This calculation was carried out to calculate both $T_{\text {obs }}$ and $T_{\text {new }}$ when $Y_{j}$ were the observed and the simulated trap specific encounter frequencies, respectively. $T_{\text {obs }}$ and $T_{\text {new }}$ were calculated for every step in the MCMC algorithm, and the number of times $T_{\text {obs }}>T_{\text {new }}$ was calculated and divided by 
the total number of MCMC iterations to get the Bayesian p-value. Bayesian p-values near 0.5 are considered to show good model fit (Royle et al., 2014). To compare model fit for the joint analyses (CT-LT and CTMx-LT), dual, independent Bayesian p-values were calculated for both of the camera and live trapping datasets (hereafter referred to as the "CT-specific" and "LT-specific" Bayesian p-values).

MCMC run information. All MCMC analyses used 100,000 iterations and an additional 10,000 iteration burn in period. The following tuning parameters were used: $\alpha_{0}=1, \lambda_{0}=0.5, \sigma=2$, and $\boldsymbol{s}_{i}=1$. Initial parameter values were chosen at the start of the MCMC from the distributions: $\alpha_{0} \sim \operatorname{Unif}(0.1,2) ; \lambda_{0} \sim \operatorname{Unif}(0.2,5)$; $\sigma \sim \operatorname{Unif}(10,30) ;$ and $\psi \sim \operatorname{Unif}(0.05,0.4)$. An $M$ value of 75 was used for all model implementations. This decision was made based on the minimum number known alive (MNKA) estimates obtained from the live trapping data. Since the purpose of the analysis was to assess the effectiveness of the $\mathrm{SC}$ model, estimates of $N$ that ranged too far beyond the MNKA values were considered to be unusable. All state-spaces, $S$, were defined by adding a $30 \mathrm{~m}$ buffer around the minimum convex polygon of the given trapping grid. 


\section{Results}

\section{Live Trapping Results}

The three most frequent species captured during live trapping were brush mice (178 capture instances), Heermann's kangaroo rat (164 capture instances), and pocket mice (155 capture instances; Table 2). Other identified species caught were much less frequent $(\mathrm{n}=7-24)$ and included: narrow-faced kangaroo rat, desert woodrat (Neotoma lepida), harvest mice, pinyon mice, and North American deer mice (Peromyscus maniculatus; hereafter, deer mice). Twenty-five individual Heermann's kangaroo rats were tagged, and five narrow-faced kangaroo rats were tagged. Observations of the narrow-faced kangaroo rat were largely (7 of 8) restricted to the chamise-buckbrush chaparral on the northwest-facing slopes. All of the narrow-faced kangaroo rats were observed during sessions S1-S6. Heermann's kangaroo rats were primarily (153 of 164) observed on the valley floor and occasionally (11 of 164) observed on the southeast facing slopes. Individual captures of Heermann's kangaroo rats were highest during S1 and S2 when 11 and 13 individuals were captured, respectively (Table 2). During sessions S3-S9 the number of unique individuals captured fell to three to five per session.

During the final spring session, S10, the number of unique individuals captured recovered to seven and included two juveniles. Although two females on the southern end of the site were observed in a post-copulatory state in March during session S9 and a female gave birth during session S8, male kangaroo rats were not captured between October 2014 and March 2015. 
Table 2. Summary of live trapping statistics for all species captured including: the number of sessions observed, the number of unique capture instances, total tags used, and the number of individuals that died. Sessions which were removed (R1, R2) are highlighted in dark grey. The gap in data collection is shown as a dark band.

\begin{tabular}{|c|c|c|c|c|c|c|c|c|c|c|c|c|c|c|c|c|c|c|}
\hline \multirow[b]{4}{*}{$\begin{array}{l}\text { Common } \\
\text { Name }\end{array}$} & \multirow[b]{4}{*}{$\begin{array}{l}\text { Latin } \\
\text { Name }\end{array}$} & \multirow[b]{4}{*}{$\begin{array}{l}\text { Sessions } \\
\text { Observed }\end{array}$} & \multirow[b]{4}{*}{$\begin{array}{c}\text { Capture } \\
\text { Instances }\end{array}$} & \multirow[b]{4}{*}{$\begin{array}{l}\text { Total } \\
\text { Tags }\end{array}$} & \multirow[b]{4}{*}{ Deaths } & \multicolumn{13}{|c|}{ Unique Inidividuals Captured Per Session } \\
\hline & & & & & & \multicolumn{6}{|c|}{2014} & \multicolumn{7}{|c|}{2015} \\
\hline & & & & & & $\begin{array}{c}\text { Apr } \\
3-7\end{array}$ & $\begin{array}{l}\text { May } \\
9-12\end{array}$ & \multirow{2}{*}{ Gap } & \multirow{2}{*}{\begin{tabular}{|c|}
$\begin{array}{c}\text { Sep } \\
12-15\end{array}$ \\
$R 1$
\end{tabular}} & \multirow{2}{*}{\begin{tabular}{|c|}
$\begin{array}{c}\text { Oct } \\
17-20\end{array}$ \\
$S 3$ \\
\end{tabular}} & \multirow{2}{*}{\begin{tabular}{c|}
$\begin{array}{l}\text { Nov } \\
7-10\end{array}$ \\
$S 4$
\end{tabular}} & \multirow{2}{*}{$\begin{array}{c}\text { Dec } \\
5-8\end{array}$} & \multirow{2}{*}{$\begin{array}{r}J a n \\
2-5 \\
S 6\end{array}$} & \multirow{2}{*}{\begin{tabular}{|c|} 
Jan 30 \\
Feb 2 \\
$S 7$
\end{tabular}} & \multirow{2}{*}{$\begin{array}{c}\text { Feb 27 } \\
\text { Mar 2 } \\
\text { S8 }\end{array}$} & \multirow{2}{*}{\begin{tabular}{|c|}
$\begin{array}{c}M a r \\
27-30\end{array}$ \\
$S 9$
\end{tabular}} & \multirow{2}{*}{\begin{tabular}{|c|}
$\begin{array}{c}A p r \\
23-27\end{array}$ \\
$S 10$
\end{tabular}} & \multirow{2}{*}{$\begin{array}{c}\begin{array}{c}\text { May } \\
22-25\end{array} \\
R 2\end{array}$} \\
\hline & & & & & & $S 1$ & $S 2$ & & & & & & & & & & & \\
\hline $\begin{array}{l}\text { California } \\
\text { Pocket Mouse }\end{array}$ & $\begin{array}{l}\text { Chaetodipus } \\
\text { californicus }\end{array}$ & 12 & 155 & N/A & 1 & 8 & 6 & & 5 & 15 & 14 & 0 & 0 & 4 & 6 & 7 & 5 & 4 \\
\hline $\begin{array}{l}\text { Unidentified } \\
\text { Kangaroo Rat }\end{array}$ & $\begin{array}{l}\text { Dipodomys } \\
?\end{array}$ & 1 & 1 & 0 & 0 & 1 & 0 & & 0 & 0 & 0 & 0 & 0 & 0 & 0 & 0 & 0 & 0 \\
\hline $\begin{array}{l}\text { Heermann's } \\
\text { Kangaroo Rat }\end{array}$ & $\begin{array}{l}\text { Dipodomys } \\
\text { heermanni }\end{array}$ & 13 & 164 & 25 & 2 & 13 & 11 & & 3 & 5 & 5 & 3 & 4 & 4 & 4 & 4 & 7 & 5 \\
\hline $\begin{array}{l}\text { Narrow-faced } \\
\text { Kangaroo Rat }\end{array}$ & $\begin{array}{l}\text { Dipodomys } \\
\text { venustus }\end{array}$ & 6 & 8 & 5 & 0 & 2 & 2 & & 0 & 1 & 1 & 0 & 1 & 0 & 0 & 0 & 0 & 0 \\
\hline Desert Woodrat & $\begin{array}{l}\text { Neotoma } \\
\text { lepida }\end{array}$ & 5 & 7 & 4 & 0 & 1 & 1 & & 0 & 0 & 1 & 0 & 0 & 0 & 0 & 0 & 0 & 1 \\
\hline $\begin{array}{l}\text { Unidentified } \\
\text { Deer Mice }\end{array}$ & $\begin{array}{l}\text { Peromyscus } \\
?\end{array}$ & 16 & 33 & 12 & 2 & 0 & 0 & & 1 & 0 & 1 & 1 & 0 & 0 & 1 & 1 & 4 & 3 \\
\hline Brush Mouse & $\begin{array}{l}\text { Peromyscus } \\
\text { boylii }\end{array}$ & 13 & 178 & 54 & 0 & 9 & 8 & & 2 & 2 & 4 & 7 & 7 & 10 & 13 & 10 & 8 & 8 \\
\hline $\begin{array}{l}\text { North American } \\
\text { Deer Mouse }\end{array}$ & $\begin{array}{l}\text { Peromyscus } \\
\text { maniculatus }\end{array}$ & 7 & 16 & 3 & 3 & 3 & 0 & & 0 & 0 & 0 & 0 & 0 & 0 & 0 & 0 & 0 & 0 \\
\hline Pinyon Mouse & $\begin{array}{l}\text { Peromyscus } \\
\text { truei }\end{array}$ & 4 & 9 & 3 & 1 & 2 & 1 & & 0 & 0 & 0 & 0 & 0 & 0 & 0 & 0 & 0 & 0 \\
\hline $\begin{array}{l}\text { Western Harvest } \\
\text { Mouse }\end{array}$ & $\begin{array}{l}\text { Reithrodontomys } \\
\text { megalotis }\end{array}$ & 8 & 24 & N/A & 1 & 3 & 0 & & 1 & 4 & 1 & 5 & 3 & 1 & 3 & 0 & 0 & 0 \\
\hline $\begin{array}{l}\text { Deer Mice }+ \\
\text { Harvest Mice }\end{array}$ & $\begin{array}{l}\text { Peromyscus }+ \\
\text { Reithrodontomys }\end{array}$ & N/A & 260 & N/A & 7 & 17 & 9 & & 4 & 6 & 6 & 13 & 10 & 11 & 17 & 11 & 12 & 11 \\
\hline Totals: & $12^{*}$ & 13 & 599* & 106 & 10 & 42 & 29 & & 12 & 27 & 27 & 16 & 15 & 19 & 27 & 22 & 24 & 21 \\
\hline
\end{tabular}

* 4 Additional Captures are included 1 Rufous-crowned Saprrow (Aimophila ruficeps), 1 Golden-crowned Sparrow (Zonotrichia atricapilla), 1 Dark-eyed Junco (Junco hyemalis), and 1 Rabbit (Sylvilagus sp.) 
All parameter estimates from the LT, CT-LT, and CTMx-LT sessions are given in Appendix C. Also, see Appendix A1 for exact live trap locations and capture histories as well as Appendix D for home range center estimates. Live trapping modal density estimates from both the EP and the HN detection functions were nearly identical. Modal density estimates during S1 and S2 (April and May 2014) ranged from 3.29 N/ha to 4.07 N/ha. Modal density estimates declined in the Fall (October - December 2014) to 1.55 $\mathrm{N} /$ ha in $\mathrm{S} 3,1.50 \mathrm{~N} /$ ha in $\mathrm{S} 4$, and $0.76 \mathrm{~N} /$ ha in $\mathrm{S} 5$ when a heavy rainstorm occurred during the first night of trapping. Density estimates for S6 (January) increased to $1.52 \mathrm{~N} / \mathrm{ha}$ after the low estimate from S5. The session S6 estimates also had an upper credible interval of 2.79 N/ha, which was higher than any credible interval from sessions S3-S5. Session S6 was also the last time a $D$. venustus individual, T\#614, was observed. After this point, only four $D$. heermanni females were observed for the next three sessions, S7-S9, and they were captured on almost every trapping night. Sessions S7-S9 (January-March) had modal density estimates of $1.03-1.04 \mathrm{~N} /$ ha and no range in the $95 \%$ credible intervals, with the exception of session S9-HN where the upper CI rose to $1.28 \mathrm{~N} / \mathrm{ha}$. Modal density rose in S10 (April) to $2.08 \mathrm{~N} /$ ha when two juveniles, one male and one female, were observed in the range of female $\mathrm{T} \# 626$. In session $\mathrm{S} 10$ the first adult male, T\#522, seen since session S3 (October), and a female, T\#416, not observed since session S4 (November) were observed.

Estimates of the home range radius parameter, $\sigma$, were similar for all LT sessions, except in S6 when estimates were notably low (see Figure 4a). During session S6, modal $\sigma$ values were $5.24 \mathrm{~m}$ and $9.4 \mathrm{~m}$ for the $\mathrm{EP}$ and $\mathrm{HN}$ detection functions, respectively. 

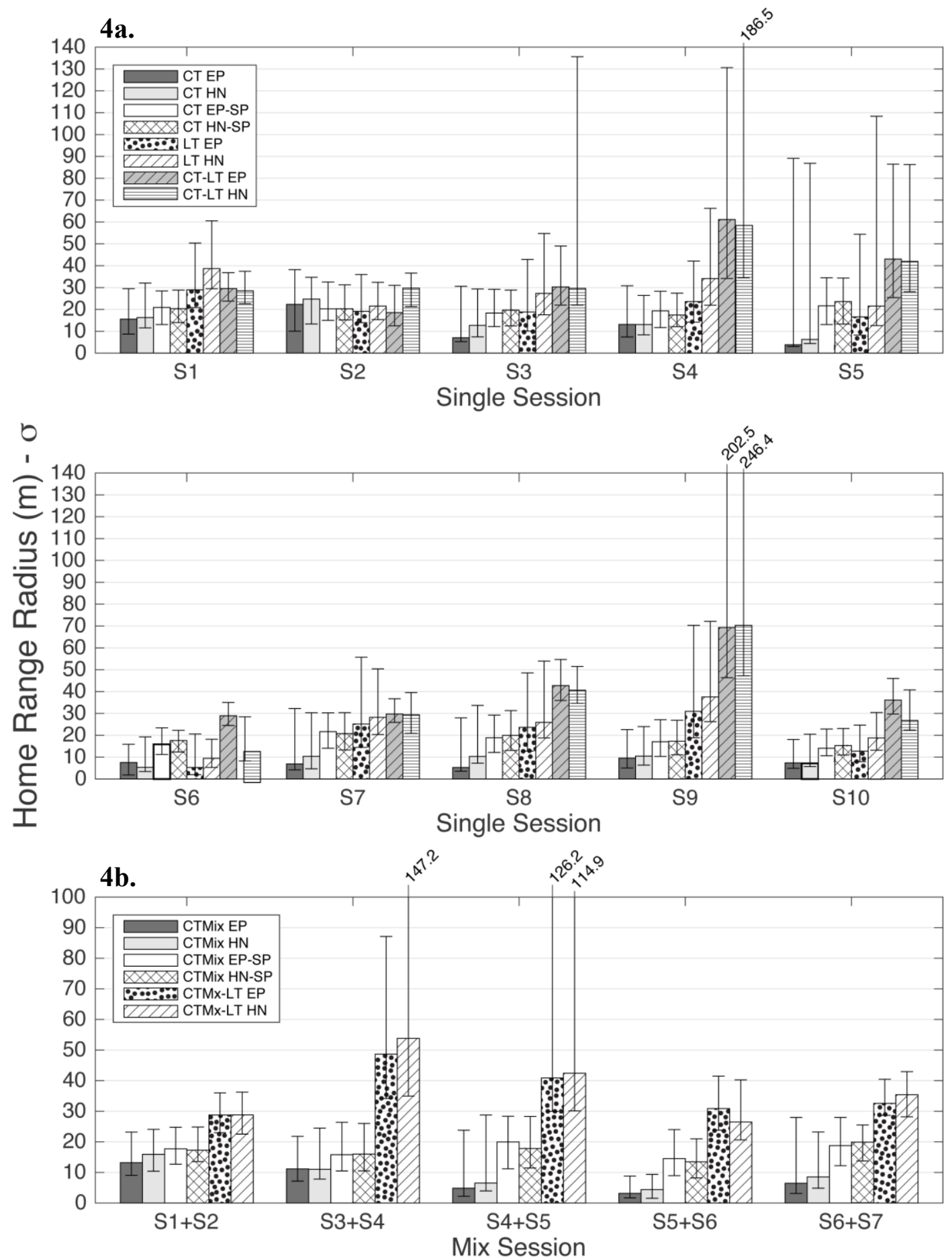

Figures $4 a-4 b$. Estimates of the home range radius parameter, $\sigma$, from the the singlesession (4a) and mixed-session (4b) datasets. Bars represent modal estimates. Error bars depict upper and lower credible intervals calculated by $95 \%$ highest density interval. Anomalously high values are displayed above the figures. 
These values were considerably lower than the rest of the sessions, where the mean $\sigma$ was $20.5 \mathrm{~m}( \pm 7.72 \mathrm{~m} \mathrm{SD})$ and $26.3 \mathrm{~m}( \pm 9.01 \mathrm{SD})$ for the EP and $\mathrm{HN}$ functions, respectively. Sessions S6's low $\sigma$ estimates coincided with high abundance estimates and more frequent home range center estimates outside the trapping grid or where trap spacing was wider than normal. $\mathrm{HN}$ function estimates of $\sigma$ were higher on average than the EP function estimates, though the credible intervals usually overlapped.

Parameter estimates for $\alpha_{0}$ generally increased over the course of the study (Figure 5a). Estimates of $\alpha_{0}$ were notably low during S1 (April) and to a lesser extent in S2 (May), after which they increased over the remaining sessions. Parameter estimates of $\alpha_{0}$ from sessions S7 and S8 (January-February) were anomalously high, and MCMC time-series plots showed no convergence, with chains trending continually upwards. During these sessions every individual captured was captured on all three trap nights. In every session except S8 (February-March), EP function $\alpha_{0}$ estimates were higher than those from the HN detection function.

Home range center estimates under the LT model generally aligned with the habitat preferences of both species (see Appendix D). Home range centers estimates for Heermann's kangaroo rat were typically on the valley floor or the southeast-facing slopes, and estimates were on the northwest-facing slopes for the narrow-faced kangaroo rat. Individual kangaroo rats were frequently observed at the same trap night after night (Appendix A1), which gave limited information on individual roaming behavior. Home ranges typically remained constant between sessions. With the exception of T\#622, all four females held stable home ranged during sessions S7-S9 (January-March). 

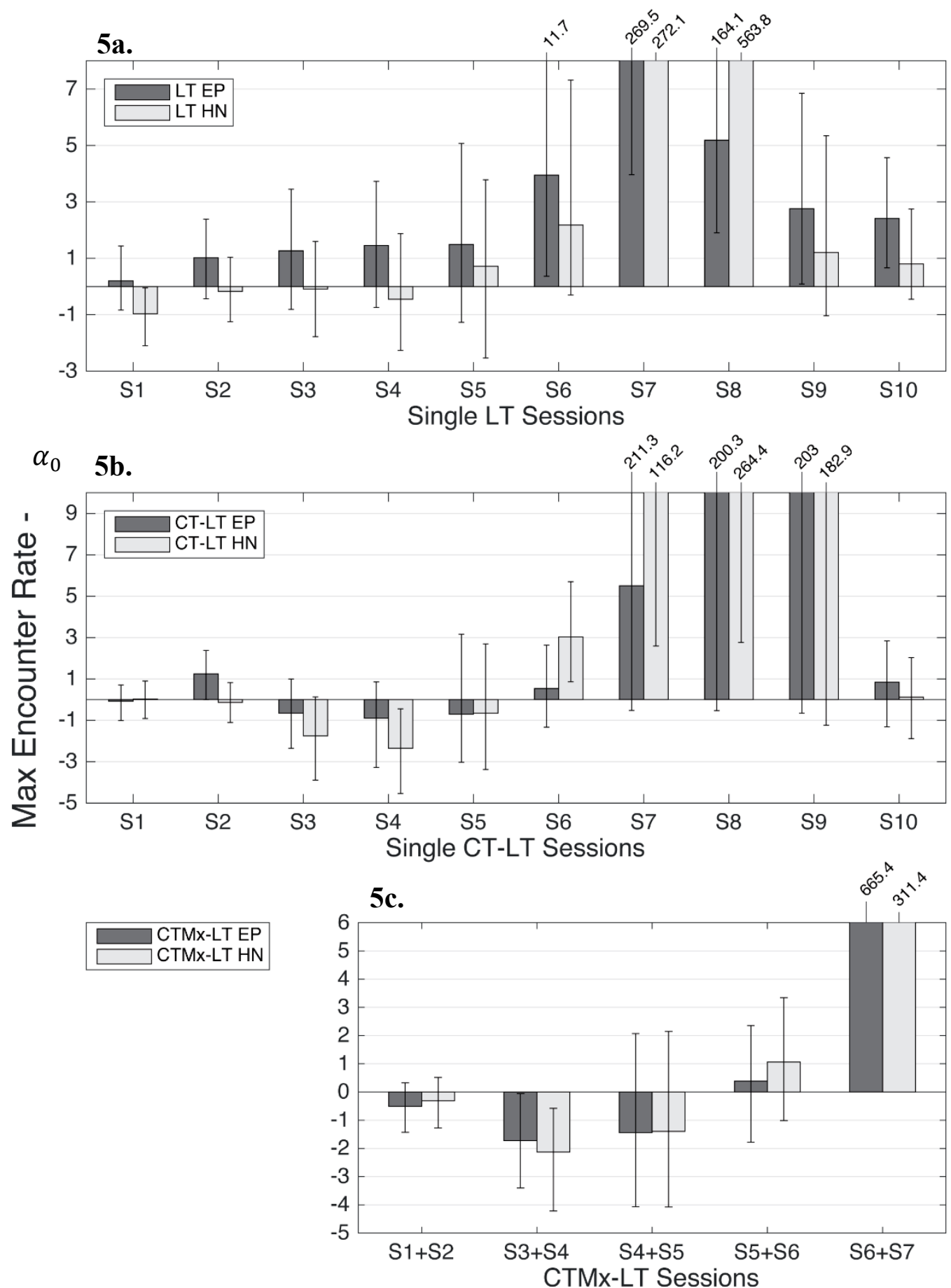

Figures $5 a-5 c$. Estimates of the multinomial capture rate parameter, $\alpha_{0}$, from the LT (5a), CT-LT (5b) and CTMx-LT (5c) datasets. Bars represent modal estimates. Error bars depict upper and lower credible intervals calculated by $95 \%$ highest density interval. Anomalously high values are displayed above the figures. Value listed is the upper credible interval. 
The LT Bayesian p-values showed moderate model fit for the EP function and poorer fit for the HN function (Figure 6). For sessions S1, S2, S6, and S7, the EP function Bayesian p-values were close to 0.5 , while the other sessions ranged from 0.23 to 0.39 . Fit values for the HN function were considerably lower. Sessions S2, S6, and S7 HN fit values were close to 0.5 , but the remainder of the sessions ranged from 0.08 to 0.33 .

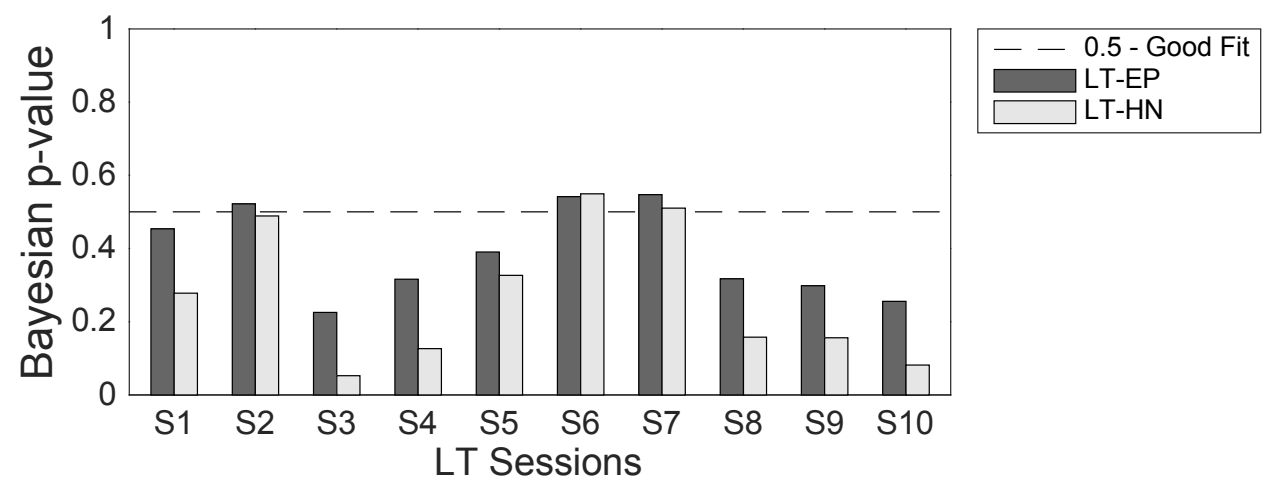

Figure 6. Bar graph comparing Bayesian p-values from the LT dataset. The dashed line shows a value of 0.5 , which is representative of good model fit.

\section{Camera Trapping Results}

Camera trapping produced a large amount of data, and an estimated 2.42 million images were collected over the course of the project. Of those, 1.42 million daytime images were excluded from analysis, and 550,000 additional images were excluded by the motion detection routine. This left approximately 450,000 images to be reviewed manually, of which 55,723 images (ca. 2.3\%) actually contained animals. A total of 3,507 triggered, 479 interval, and 1510 missed capture events were recorded during sessions S1-S10. A wide range of species were observed in camera trap images (Tables 3 and 4). 
Table 3. Camera trap summary information for the most frequent species captured. Session totals are based on the $2 \mathrm{~h}$ hit window. Western harvest mice (Reithrodontomys megalotis) and North American deer mice (Peromyscus maniculatus) were difficult to differentiate in camera trap images. As such, the summary information listed for these two species is a best guess by the reviewer.

\begin{tabular}{|c|c|c|c|c|c|c|c|c|c|c|c|c|c|c|}
\hline Common Name & Species Name & $\begin{array}{c}\text { Total } \\
\text { Images }\end{array}$ & $\begin{array}{c}\text { Total } 15 \mathrm{~s} \\
\text { Capts }\end{array}$ & $\begin{array}{l}\text { Total } 2 \text { h } \\
\text { Capts }\end{array}$ & S1 & S2 & S3 & S4 & S5 & S6 & S7 & S8 & S9 & S10 \\
\hline Kangaroo Rat & Dipodomys spp. & 18990 & 2024 & 866 & 192 & 170 & 52 & 92 & 19 & 42 & 42 & 87 & 60 & 110 \\
\hline California Pocketmouse & Chaetodipus californicus & 3487 & 459 & 253 & 22 & 25 & 43 & 99 & 6 & 0 & 12 & 5 & 12 & 29 \\
\hline Western Harvest Mouse * & Reithrodontomys Megalotis & 437 & 62 & 35 & 0 & 0 & 2 & 5 & 19 & 0 & 0 & 2 & 2 & 5 \\
\hline Deermouse $*$ & Peromyscus spp. & 5841 & 931 & 565 & 29 & 5 & 65 & 128 & 100 & 39 & 62 & 69 & 23 & 45 \\
\hline Deer Mice + Harvest Mice & Pero. + Reithro. & 6278 & 993 & 600 & 29 & 5 & 67 & 133 & 119 & 39 & 62 & 71 & 25 & 50 \\
\hline Black-tailed Jackrabbit & Lepus californicus & 7111 & 689 & 372 & 6 & 22 & 25 & 12 & 25 & 7 & 15 & 85 & 63 & 112 \\
\hline Desert Cottontail & Sylvilagus auduboni & 3564 & 303 & 105 & 13 & 42 & 14 & 2 & 10 & 6 & 3 & 6 & 2 & 7 \\
\hline Raccoon & Procyon lotors & 387 & 100 & 31 & 5 & 0 & 17 & 0 & 0 & 0 & 1 & 2 & 2 & 4 \\
\hline Bobcat & Lynx rufus & 222 & 47 & 38 & 7 & 4 & 0 & 3 & 10 & 1 & 4 & 2 & 7 & 0 \\
\hline Grey Fox & Urocyon cinereoargenteus & 146 & 29 & 15 & 3 & 3 & 3 & 1 & 4 & 0 & 0 & 1 & 0 & 0 \\
\hline Mule Deer & Odocoileus hemionus & 1592 & 125 & 73 & 3 & 7 & 7 & 19 & 16 & 0 & 3 & 5 & 8 & 5 \\
\hline Western Toad & Anaxyrus boreas & 168 & 74 & 27 & 27 & 2 & 0 & 6 & 4 & 1 & 5 & 6 & 1 & 3 \\
\hline
\end{tabular}


Table 4. List of uncommon species captured during camera trapping that were not listed in the capture histories in Table 3.

\begin{tabular}{|l|l|}
\hline Common Name & Species Name \\
\hline Botta's Pocket Gopher & Thomomys bottae \\
Vole & Microtus spp. \\
Merriams Chipmunk & Tamias merriami \\
Brush Rabbit & Syvilagus bachmani \\
Striped Skunk & Mephitis mephitis \\
Badger & Taxidea taxus \\
White-crowned Sparrow & Zonotrichia leucophrys \\
Golden-crowned Sparrow & Zonotrichia atricapilla \\
Fox Sparrow & Passerella iliaca \\
California Quail & Callipepla californica \\
Red-shouldered Hawk & Buteo lineatus \\
Mourning Dove & Zenaida macroura \\
California Thrasher & Toxostoma redivivum \\
Western Scrub Jay & Aphelocoma californica \\
Western Fence Lizard & Sceloporus occidentalis \\
San Joaquin Coachwhip & Masticophis flagellum \\
Coast Horned Lizard & Phrynosoma coronatum \\
California Whiptail & Aspidoscelis tigris munda \\
Pacific Tree Frog & Pseudacris regilla \\
Unkown Newt/Salamnder & Salamandridae \\
\hline
\end{tabular}

Many larger mammals were observed including predators such as grey fox (Urocyon cinereoargenteus), bobcat (Lynx rufus), badger (Taxidea taxus), striped skunk (Mephitis mephitis), and mountain lion (Puma concolor). Since the 5 min interval images were not dependent on heat signatures, many ectotherms including amphibians, reptiles, and invertebrates were also observed. The most frequently observed ectotherm was the western toad (Bufo borealis). Other ectothermic species such as salamanders, frogs, and 
insects were also observed, but they were difficult to reliably identify to species using low-resolution images.

The hit window that produced the optimal mean to variance ratio was $2 \mathrm{~h}$ (Figure 7). Using this hit window, the number of unique events per trap night was calculated for the most prevalent species (Table 5). This reduced the average number of captures used in analysis by about $50 \%$.

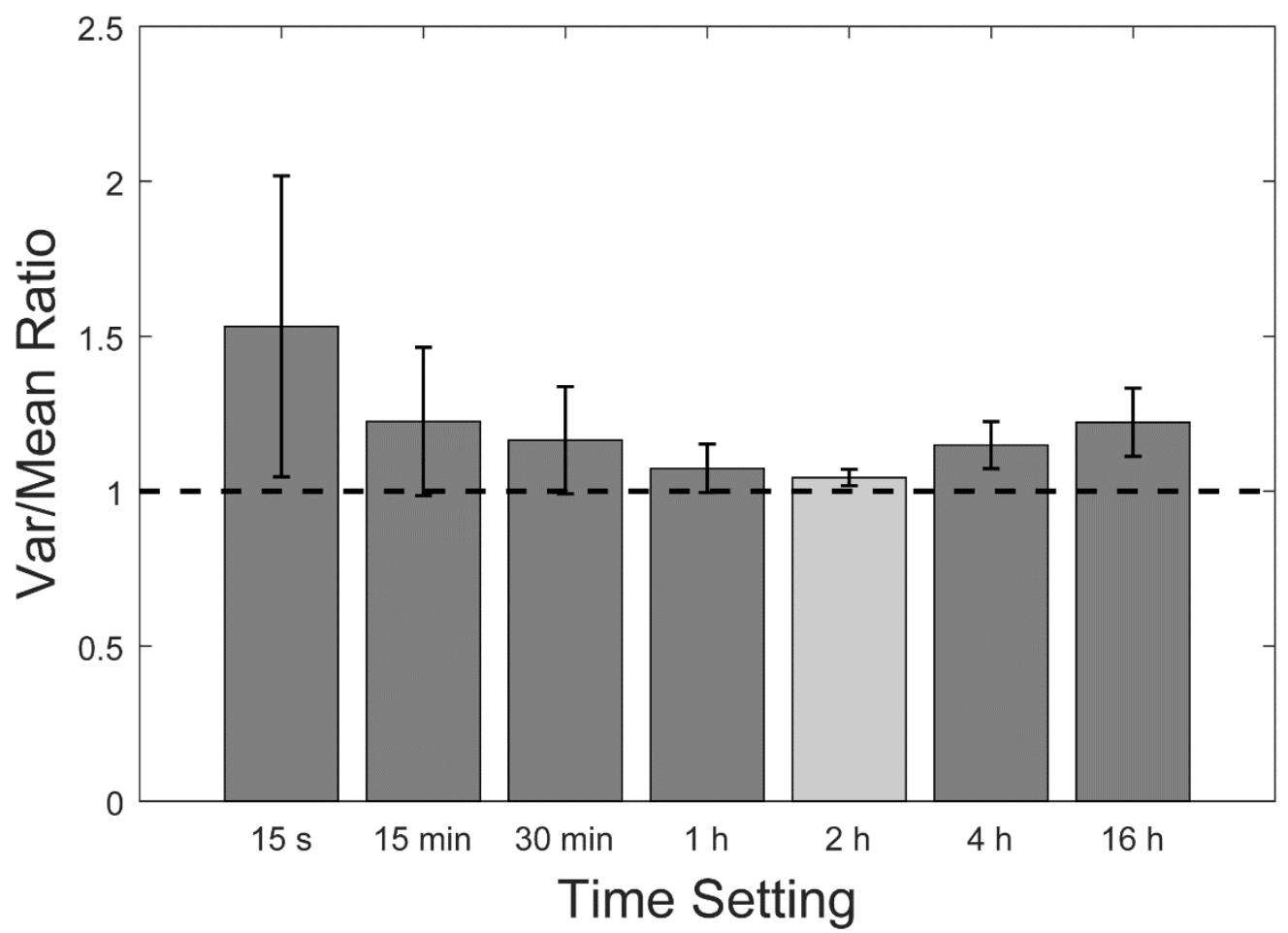

Figure 7. Bar graph showing the results of the optimal hit window analysis. For equivalent scaling across hit windows, bars heights were calculated as $e^{|\log (r)|}$, where $r$ is the variance-to-mean ratio for each hit window averaged over all sessions and cameras. Error bars depict a standard deviation of averaged variance-to-mean ratios. The bar representing the hit window with the optimal mean-to-variance ratio, $2 \mathrm{~h}$, is highlighted in light grey. 
In total, the number of missed endothermic capture events was estimated at $20.0 \%$ of the total potential captures events (Table 5). The effective number of missed captures, the number of captures the cameras would have missed without the interval setting, was $24.9 \%$. The percentage of misses per session varied greatly. The highest percentages were in the Spring and late Fall sessions, S1, S2, S3, and S10; the lowest percentages were in the late Fall and Winter sessions, S4-S9. The regression on endothermic miss rate and mean nighttime temperature had a significant positive relationship $(\beta=2.95$, $p_{\beta}=0.009, \alpha=1.256, p_{\alpha}=0.852, \mathrm{R}^{2}=0.598 ;$ Figure $\left.8 \mathrm{a}\right)$. For the eight cameras with the most nights active, the median miss-to-capture ratio ranged from 0.172 to 0.477 , with an average rate of 0.291 . Since homogeneity of variances could not be assumed (Bartlett's Test, $\chi^{2}=60.4$, d.f. $=7, \mathrm{p}<0.001$ ), a Kruskal-Wallis test was used. The test did not find a significant difference in miss rates between camera units $\left(\chi^{2}=2.98, \mathrm{p}=0.887\right)$.

Table 5. List of summary statistics for each of the CT sessions including information on the number of triggered captures, the number of interval captures, miss rates, average temperatures, camera effort, and amount of camera outages.

\begin{tabular}{|l|c|c|c|c|c|c|c|c|c|c|c|}
\hline \multicolumn{1}{|c}{ Value } & Total & S1 & S2 & S3 & S4 & S5 & S6 & S7 & S8 & S9 & S10 \\
\hline Triggered Captures & 3507 & 440 & 530 & 300 & 498 & 303 & 175 & 181 & 365 & 259 & 456 \\
Endo-Interval Capts & 230 & 36 & 47 & 26 & 11 & 25 & 13 & 14 & 18 & 13 & 27 \\
Ecto-Interval Capts & 249 & 83 & 4 & 0 & 5 & 43 & 10 & 30 & 48 & 9 & 17 \\
Misses All & 1510 & 217 & 275 & 205 & 122 & 144 & 55 & 42 & 135 & 52 & 263 \\
Endo Misses & 934 & 66 & 253 & 205 & 84 & 53 & 31 & 13 & 37 & 31 & 161 \\
Endo Miss \% & $20.0 \%$ & $12.1 \%$ & $30.5 \%$ & $38.6 \%$ & $14.1 \%$ & $13.9 \%$ & $14.2 \%$ & $6.4 \%$ & $8.8 \%$ & $10.2 \%$ & $25.0 \%$ \\
Effective Miss \% & $24.92 \%$ & $18.8 \%$ & $36.2 \%$ & $43.5 \%$ & $16.0 \%$ & $20.5 \%$ & $20.1 \%$ & $13.1 \%$ & $13.1 \%$ & $14.4 \%$ & $29.2 \%$ \\
Avg Temp (C) & N/A & 7.72 & 10.17 & 12.39 & 7.61 & 7.17 & 2.39 & 6.11 & 4.94 & 6.00 & 7.28 \\
Effort (Cam Days) & 4278 & 423 & 472 & 548 & 541 & 491 & 318 & 391 & 396 & 361 & 337 \\
Camera Days/Total & N/A & $81 \%$ & $87 \%$ & $99 \%$ & $98 \%$ & $99 \%$ & $93 \%$ & $100 \%$ & $97 \%$ & $98 \%$ & $88 \%$ \\
\hline
\end{tabular}



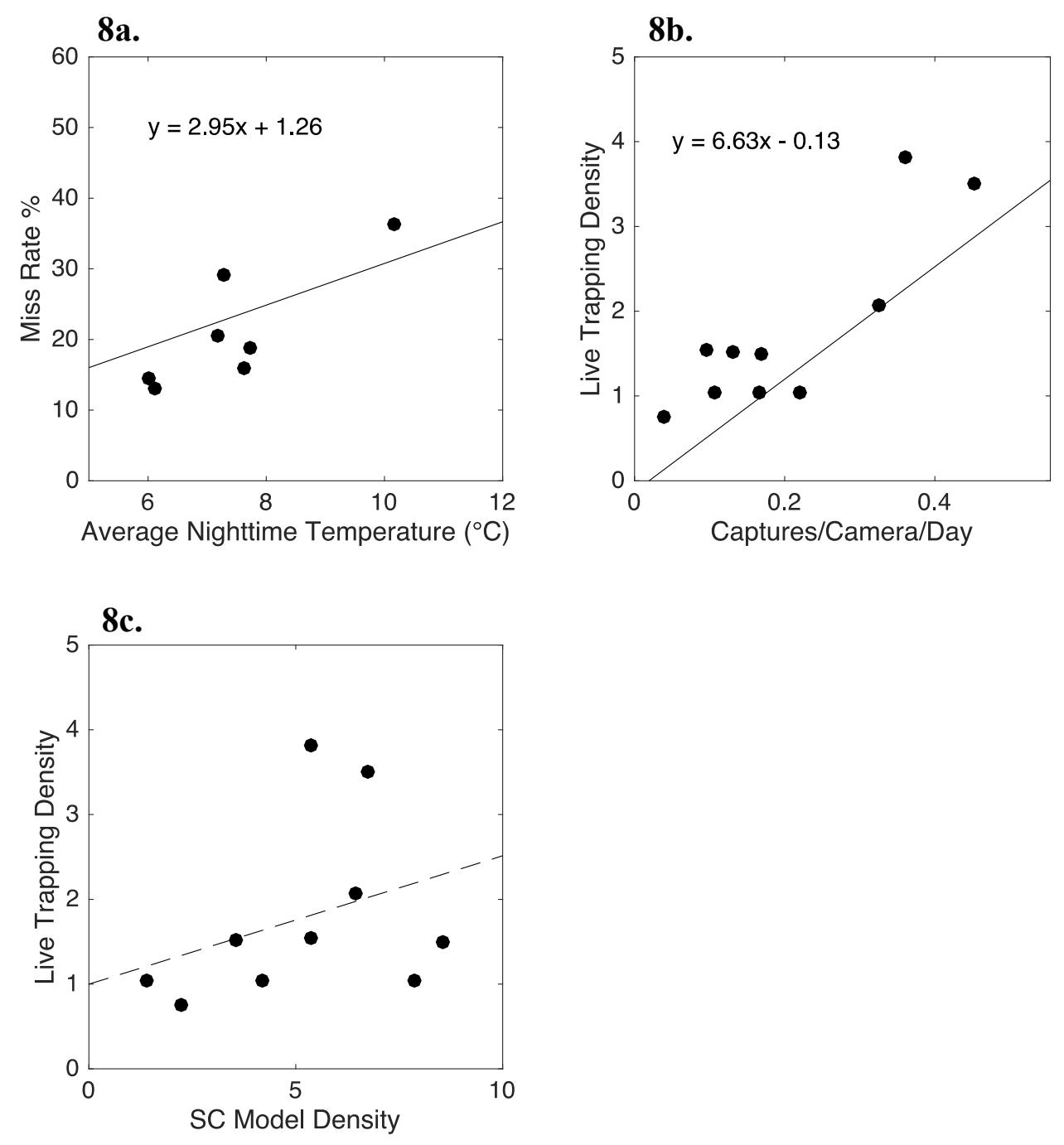

Figures $8 a-8 c$. Results of regressions comparing (8a) missed capture rate to average ambient nighttime temperatures, (8b) SC model density from the EP-SP sessions to LT density, and (8c) average daily capture rate to live trapping density. Solid lines indicate significant regressions coefficients, for which linear models are given. Dashed lines indicate non-significant regression coefficients.

A total of 18,990 images were taken of kangaroo rats, which resulted in 866 capture events using the $2 \mathrm{~h}$ hit window. Kangaroo rat capture rates fluctuated throughout the study. Rates were highest during S1 and S2, declined through S5, and then increased again steadily through S10. Results of the multiple linear regression (Figure 8b) showed 
that LT-EP density had a significant relationship with kangaroo rat camera capture rate $\left(\beta_{1}=6.63, p_{\beta_{1}}<0.001\right)$ and a slight relationship with endothermic miss rate $\left(\beta_{2}=0.031\right.$, $\left.p_{\beta_{2}}=0.082\right)$, though the intercept was not significant $\left(p_{\alpha}=0.742, \alpha=-0.128\right)$. When miss rate was removed from the regression, $R^{2}$ values fell from 0.799 to 0.720 . The regression that compared LT density to the best CT density estimate, EP-SP, and endothermic miss rate (Figure 8c) did not show a significant relationship (all $p>0.05$ ).

Results of the CT-LT model provided the best parameter estimates to compare the CT dataset's SC model abundance estimates against. Although some modal abundances from the SC model were close to those estimated by the CT-LT data, many showed wide credible intervals (Figures 9a-9b). Differences between the modal CT abundance estimates and the CT-LT estimates were greatest during sessions S1, S3, S4, S9, and S10. Here, credible intervals had little overlap with the CT-LT modal abundance estimates. Upper credible intervals and even modal estimates for these sessions often included the upper abundance limit, $M=75$; this indicates that the model would have estimated higher abundances had $M$ been set higher. Modal abundance estimates were much closer to the CT-LT values during sessions S2 and S5-S8. None of these sessions had credible intervals that reached $\mathrm{M}$, and though a positive bias was noted, the credible intervals regularly included the CT-LT abundance value. Application of a prior on $\sigma$ increased model precision, and only S1 HN-SP had a credible interval that extended up to $M$. Mean credible interval ranges were $62.3(\mathrm{EP})$ and $63.5(\mathrm{HN})$ for the implementations without a prior on $\sigma$, and they were $37.1(\mathrm{EP})$ and $41.8(\mathrm{HN})$ for the implementations with a prior. 

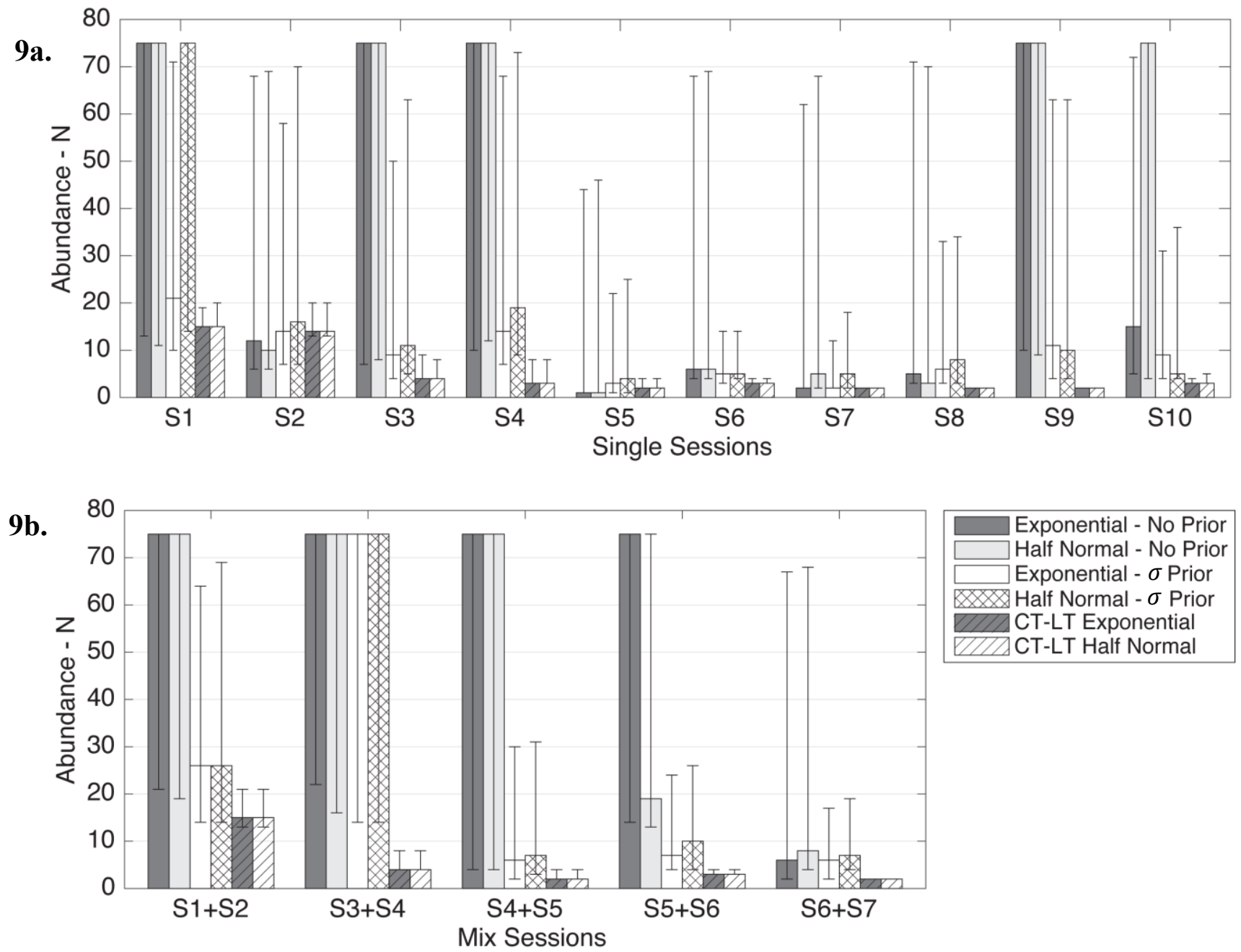

Figures $9 a-9 b$. Bar graphs comparing the estimates of abundance, $N$, from (9a) the CT and CT-LT datasets and (9b) the CTMix and CTMx-LT datasets. Bars represent modal estimates. Error bars depict upper and lower credible intervals calculated by $95 \%$ highest density interval. 
The implementation with the best coverage was EP-SP, where six of ten sessions covered the abundance estimate from the CT-LT data. The EP-SP implementation also had the smallest average credible range. All of these factors made EP-SP the best implementation of the SC model in terms of abundance estimation.

Mean CT-LT estimates of the home range size parameter, $\sigma$, were $38.9 \mathrm{~m}$ (EP) and $37.0 \mathrm{~m}(\mathrm{HN})$; these means were more than three times larger than those from the CT data, where the means were $9.8 \mathrm{~m}(\mathrm{EP})$ and $11.7 \mathrm{~m}(\mathrm{HN}$; Figure 4a). Modal $\sigma$ estimates were high during sessions S1 and S2, with EP values of $15.6 \mathrm{~m}$ and $22.3 \mathrm{~m}$ and $\mathrm{HN}$ values of $16.3 \mathrm{~m}$ and $24.7 \mathrm{~m}$. During sessions S3-S10, $\sigma$ estimates were low; EP $\sigma$ values ranged from $3.9 \mathrm{~m}$ to $13.1 \mathrm{~m}$, and $\mathrm{HN}$ values ranged from $5.3 \mathrm{~m}$ to $13.1 \mathrm{~m}$. As expected, parameter estimates were more consistent when the prior was placed on $\sigma$; EP values ranged from $14.1 \mathrm{~m}$ to $21.7 \mathrm{~m}$, and $\mathrm{HN}$ values ranged from $15.3 \mathrm{~m}$ to $23.6 \mathrm{~m}$. The gamma distribution used as the $\sigma$ prior maximizes at 22, but the average modal values, $18.8 \mathrm{~m}$ (EP) and $19.2 \mathrm{~m}(\mathrm{HN})$, were lower than this. The credible range of the $\sigma$ estimates was typically larger when there was no prior than when the prior was in place. In session S5 the $\sigma$ estimates became polymodal and produced superficially high upper credible intervals of $86.5 \mathrm{~m}(\mathrm{EP})$ and $86.3 \mathrm{~m}(\mathrm{HN})$. The CT-LT data produced superficially high estimates of $\sigma$ during sessions $\mathrm{S} 4$ and $\mathrm{S} 9$, and to a lesser extent in S5 and S8. These trapping sessions were the same sessions where diffuse estimates of home range centers were observed. CT-LT estimates of $\sigma$ were typically larger than the LT $\sigma$ estimates, and differences as high as $38.4 \mathrm{~m}$ were observed between the two datasets. Only CT-LT session S1-HN had a notably lower $\sigma$ estimate than the LT data. 

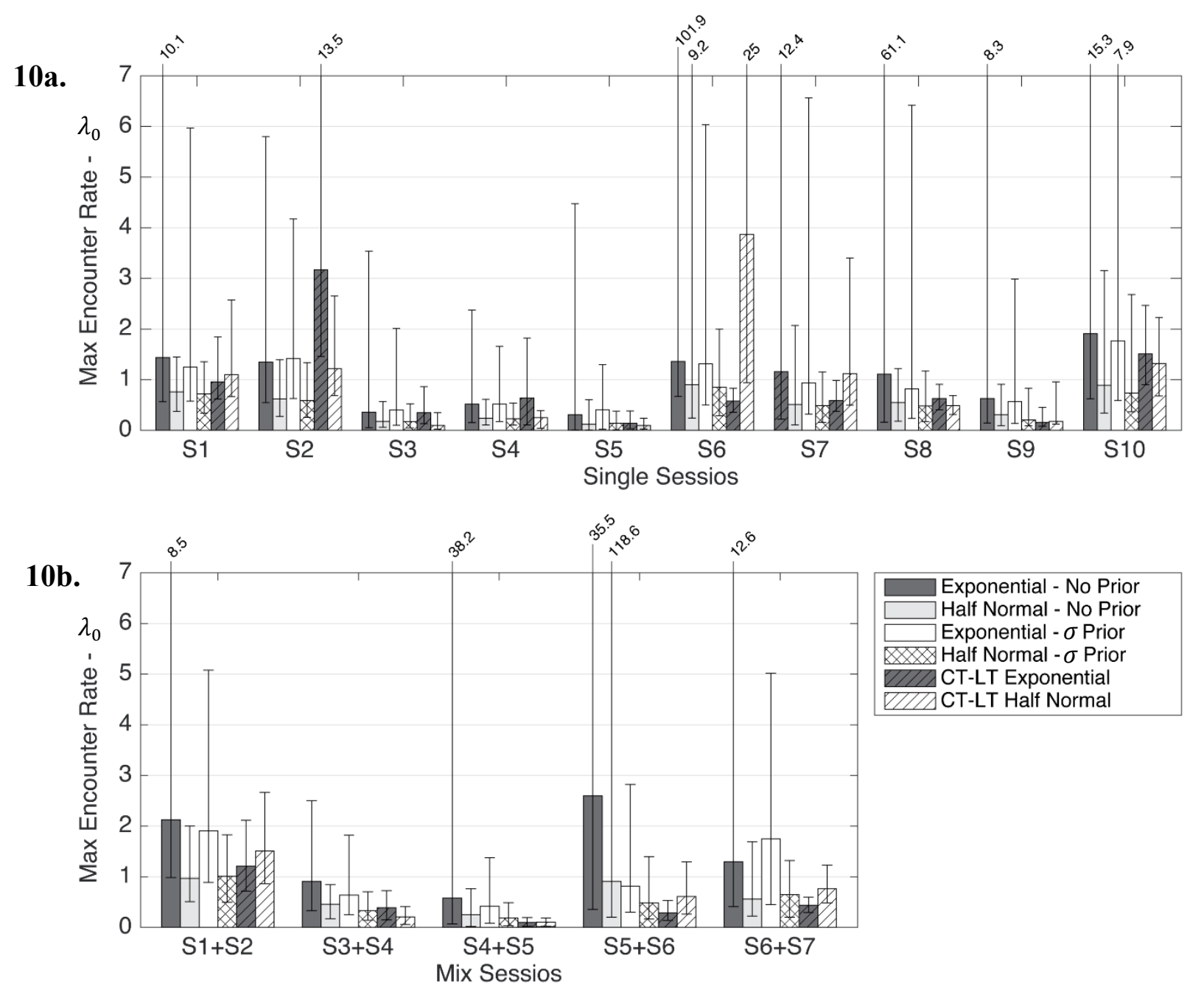

Figures $10 a-10 b$. Bar graphs comparing the estimates of the maximum capture rate parameter, $\lambda_{0}$, from (10a) the $\mathrm{CT}$ and CT-LT datasets and (10b) the CTMix and CTMx-LT datasets. Bars represent modal estimates. Error bars depict upper and lower credible intervals calculated by $95 \%$ highest density interval. 
Modal estimates and credible intervals of the maximum encounter rate parameter, $\lambda_{0}$, varied between detection functions and sessions (Figure 10a). CT-LT and CT datasets produced similar results, and having a prior on $\sigma$ did not affect $\lambda_{0}$ estimates. Using the EP function approximately doubled the average $\lambda_{0}$ estimate. Average modal $\mathrm{HN}$ function $\lambda_{0}$ values were 0.51 with no prior and 0.46 with a prior on $\sigma$, whereas average modal EP values were 1.02 with no prior and 0.94 with a prior on $\sigma$. Sessions S3, S4, S5, and S9 all had lower modal $\lambda_{0}$ estimates that ranged from 0.31 to 0.63 with the EP function and from 0.12 to 0.31 with the HN function. Sessions S1, S2, S6, S7, S8, and S10 had higher modal $\lambda_{0}$ estimates that ranged from 0.82 to 1.44 with the EP function and from 0.51 to 0.90 with the $\mathrm{HN}$ function. Notably high upper credible intervals were observed when the EP detection function was implemented without a prior on $\sigma$. Review of the MCMC time series plots showed a pattern of temporary chain divergence where $\lambda_{0}$ values rose sharply as $\sigma$ values fell to low values. This resulted in polymodal $\lambda_{0}$ estimates in cases such as session S6, and extended tails in others. This chain behavior was not as significant with a prior on $\sigma$, but the high upper bound of the EP function suggested a similar behavior.

In general, estimates of home range center locations, $\boldsymbol{s}_{i}$, were more precise when abundance estimates were more precise and when a prior was used on $\sigma$. Choice of detection function did not have a significant effect on home range center estimates, except in CT-LT session S3-EP when $\boldsymbol{s}_{i}$ estimates were much more diffuse than S3-HN. Appendix E shows density plots of home range center point estimates. One of the most notable features of the density plots is the influence that cameras with few or no captures had on the estimates of home range centers. Cameras with few or no captures produced 
distinct circles of low density $\boldsymbol{s}_{\mathbf{i}}$ estimates. Conversely, cameras with high relative capture rates and captures in areas devoid of other captures produced areas of high density $\boldsymbol{s}_{i}$ estimates. While sessions S1 and S2 had low accuracy abundance estimates, the activity center estimates from the CT data matched those from the LT data well. CT and CT-LT estimates from session S3 had strong agreement with the LT home range center estimates, with the exception of where it placed a narrow-faced kangaroo rat, T\#614. The LT data placed T\#614 in the chamise-buckbrush chaparral, whereas the CT and CT-LT data placed it on the canyon floor, where there were many camera trap captures events but no live trap captures. Session S4 had the most diffuse estimates of home range centers, and chain convergence was poor across the study area. Session S5 home range center estimates hit a singular peak with three distinct clouds emerging around it. The clouds aligned with the home range center estimates from the LT and CTLT data but the peak did not. Sessions S6 and S7 had the most accurate abundance estimates and the best convergence of specific home range centers. Four distinct convergence points emerge in S6 density plots and three in S7. Two of these points correspond to known individuals, $\mathrm{T} \# 615$ and $\mathrm{T} \# 626$. In session S6 another point was likely T\#614, which was not observed again after S6. The remaining point is an extra individual estimated by the SC model that is not accounted for in the LT data. During sessions S6 and S7, home range centers generally agreed between the CT-LT and LT datasets. Session S8 $\boldsymbol{s}_{i}$ estimates matched those from the LT dataset but were diffuse without a prior on $\sigma$. CT home range center estimates from sessions $\mathrm{S} 9$ and $\mathrm{S} 10$ had poor fit with the LT data. However, the $\boldsymbol{s}_{i}$ estimates from these sessions data are questionable 
since there was an unaccounted for male in S9 and young that were likely sharing home ranges with their mothers in S10.

CT Bayesian p-values were between 0.27 and 0.60 for every session except S1 and S10 (Figure 11a). Session S1 Bayesian p-values were all near 0, and S10 values were between 0.10 and 0.15 . Neither detection function produced better Bayesian p-values, but a prior on $\sigma$ slightly lowered them. During every CT-LT session except S2, S5, and S7, the CT-specific Bayesian p-values were 0.07 or lower; these values were considerably lower than those produced by the CT data alone. For all CT-LT sessions except for S2, the LT-specific Bayesian p-values were substantially higher than the CT-specific values.

$11 a$.
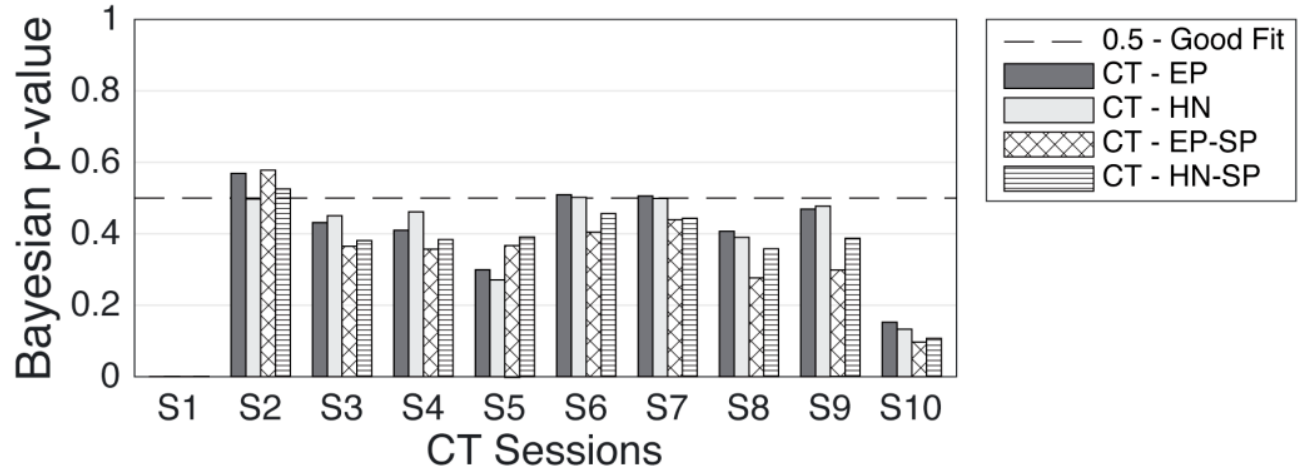

$11 b$.
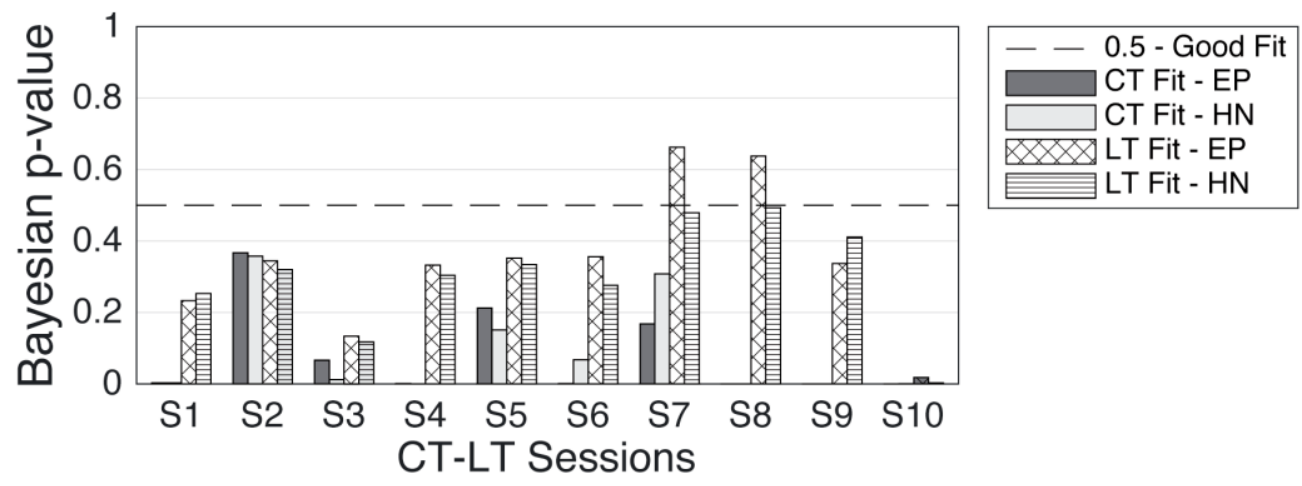

Figures 11a-11b. Bar graphs comparing Bayesian p-values from (11a) the CT datasets and (11b) the CT-LT datasets. The dashed line shows a value of 0.5 , which is representative of good model fit. For the CT-LT data, separate Bayesian p-values are given for the Live trapping (LT Fit) data and the camera trapping (CT Fit) data. 
The CTMix dataset produced mixed results for several parameters. Combining placements resulted in heterogeneous camera density due to errors made in the field, and this likely affected parameter estimation (see Appendix A3). CTMix abundance estimates were less precise and less accurate than the single-session CT results. Modal abundance estimates and credible intervals were all similar or larger than the single-session CT estimates (Figure 9b). CTMix modal abundance estimates were also larger than the CTMx-LT estimates. CTMix estimates never fell below six, but the CTMx-LT data estimated abundances as low as two and three. During mix session $\mathrm{S} 3+\mathrm{S} 4$, all four implementations resulted in modal abundance estimates at $M$ and little to no MCMC chain convergence. For every session but $\mathrm{S} 6+\mathrm{S} 7$, the implementations without a prior on $\sigma$ performed poorly. During these sessions, the upper credible intervals were at $M$, and modal abundance values were significantly larger than those from the CTMx-LT data. Without a prior on $\sigma$, all the CTMix sessions estimated low $\sigma$ values (Figure 4b). During sessions $\mathrm{S} 4+\mathrm{S} 5, \mathrm{~S} 5+\mathrm{S} 6$, and $\mathrm{S} 6+\mathrm{S} 7, \sigma$ estimates were similar to those from the $\mathrm{CT}$ dataset and ranged from $3.2 \mathrm{~m}$ to $8.5 \mathrm{~m}$. Sessions $\mathrm{S} 1+\mathrm{S} 2$ and $\mathrm{S} 3+\mathrm{S} 4$ had slightly higher values that ranged from $11.0 \mathrm{~m}$ to $15.9 \mathrm{~m}$. The alignment of session $\mathrm{S} 1+\mathrm{S} 2$ 's home range center estimates with the CTMx-LT data was notably good. Placing a prior on $\sigma$ increased the precision and accuracy of the CTMix abundance estimates but also resulted in lowered Bayesian p-values (Figure 12a). Estimates of the usage rate parameter, $\lambda_{0}$, were similar to those from the single-session CT data (Figure 10b). Review of the capture histories (Appendix A3) revealed patterns of space usage that were distinctly different from the circular distributions described by SCR models. CT-specific Bayesian p-value 
averages for the CTMx-LT (Figure 12b) data were 0.012 for the EP function and 0.015 for the HN function; LT-specific values ranged between 0.150 and 0.798 . This trend of low CT-specific Bayesian p-values in the CTMx-LT data matched the CT-LT results but was more pronounced.

12a.
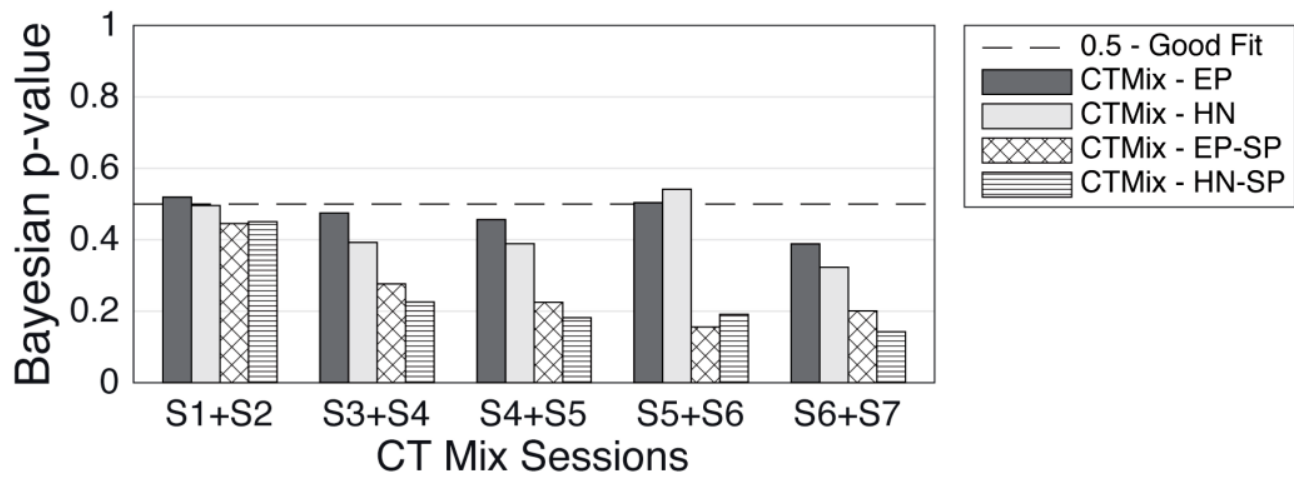

$12 b$.

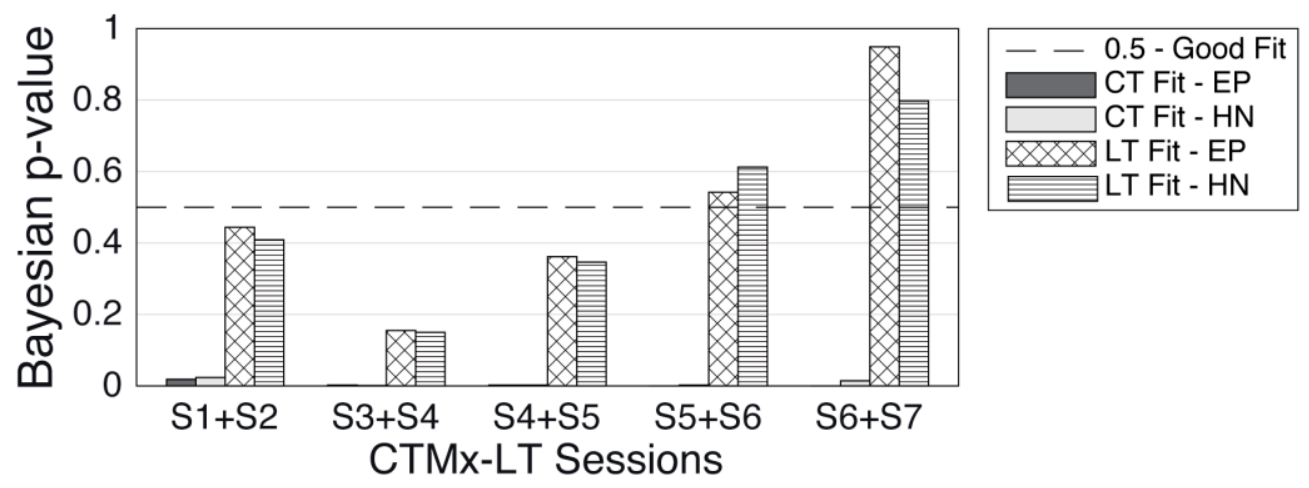

Figures 12a-12b. Bar graphs comparing Bayesian p-values from (12a) the CTMix datasets and (12b) the CTMx-LT datasets. The dashed line shows a value of 0.5 , which is representative of good model fit. For the CT-LT data, separate Bayesian p-values are given for the Live trapping (LT Fit) data and the camera trapping (CT Fit) data. 


\section{Discussion}

This is the first study in which estimates of small mammal density were produced from camera data alone. The SC model was tested against independent and robust measures of density, and these results were tracked across multiple trapping sessions. I found that the SC model can work when the underlying assumptions of the model align with the study species' spatial ecology but results can be highly sensitive to deviations from those assumptions. Comparing the camera trapping data to the live trapping data and combining the two in the CT-LT and CTMx-LT datasets provided useful insights into the relative strengths and weaknesses of both trapping methods. However, anomalies in parameter estimates were observed for nearly all model implementations due to the changing shape of the camera grid and low population density. While these anomalies make overarching conclusions about the SCR models difficult, they provide useful information on how these models respond under difficult sampling situations. Particularly sampling situations with low sample sizes, a situation frequently encountered by wildlife researchers and land managers working with minimal resources. In order to make the SC model a more viable option for density estimation, researchers should incorporate other observational and habitat related covariates to improve model precision.

\section{Live Trapping Discussion}

Kangaroo rat capture rates were high in April and May 2014 but were substantially reduced after the gap in data collection during the Summer of 2014. After this gap, the number of individual kangaroo rats at the study site remained relatively stable throughout the winter. It is unclear what caused the initial population decline, but it 
may have been caused by typical seasonal fluctuation, effects from an ongoing drought, or an impact from the raccoon attack. Based on four years of live trapping data, Fitch (1948) described the seasonal variation of a Heermann's kangaroo rat population in California's Central Valley. Though between year variation was high, Fitch observed reduced abundances between September and December and increased abundances between June and August. The Pinnacles population may have shown a seasonal pattern that was similar, but offset due to differences in climate between the two sites. The absence of trapped male kangaroo rats between October and March is surprising, and no other multi-seasonal studies conducted on Heermann's kangaroo rat have noted any sexspecific seasonal movements (Tappe, 1941; Fitch, 1948). Both the raccoon attack and trap shyness are possible explanations of why males were unobserved. Another possibility is that low densities caused males living in adjacent areas to not be viewed due to chance. Without multi-year studies, it is difficult to make strong conclusions about the sex bias observed in the live capture data.

The multinomial model used with the LT data provided reasonably consistent results throughout the study. The biggest anomaly was the over-estimate of density and the under-estimate of $\sigma$ during session $\mathrm{S} 6$, though a similar pattern was observed in other sessions. In session $\mathrm{S} 6$, the small $\sigma$ estimates were likely caused by capture locations that were tightly grouped, varying only by $10-15 \mathrm{~m}$. These small $\sigma$ estimates allowed an increased number of home range centers to be estimated around the perimeter of the trap grid, which led to increased estimates of abundance and density. This link shows how inaccurate $\sigma$ estimates can lead to an overestimation of density. Another anomaly noted 
was the low and overly-precise abundance estimate of abundance in session S5. Here, two individuals, $\mathrm{T} \# 622$ and $\mathrm{T} \# 614$, were not observed even though they were likely still present. The problems caused by these two anomalies may have been alleviated by using an open-population model that accounted for temporary immigration and changes in parameter estimates across sessions (Gardner, Reppucci, Lucherini \& Royle, 2010).

The high $\alpha_{0}$ estimates, low $\sigma$ estimates, higher home range center precision, higher upper credible intervals, and better Bayesian p-value fit for the EP function were likely related. The spired shape of the EP function (Figure 3) allowed for better fit when individuals had an affinity for specific traps. This property of the EP function resulted in higher $\alpha_{0}$ estimates, more precise home range centers, and better fit from the aggregated trap total perspective. The slightly thicker tail of the EP function allowed for smaller $\sigma$ estimates from the same dataset by maintaining higher capture probabilities farther from the home range center. In order to collect better data on space utilization using live trapping data, it may be warranted to close or move traps where individuals have been previously captured.

Sessions S7 and S8 showed anomalously high $\alpha_{0}$ estimates, which appeared to be a result of all four individuals observed being caught on all three nights. This resulted in no variation with which to estimate capture probability from and made the modal $\mathrm{N}$ estimates converge on an overly-precise solution. Indeed, this precision was not warranted since the post-copulatory state of the females in S9 was evidence of at least one male that was unaccounted for. This problem is largely a result of low sample sizes 
and could be remedied by sampling over the larger population that CR models were intended for (Otis et al., 1978; Harmsen et al., 2011).

\section{Camera Trapping Discussion}

The large volume of images produced by capturing both motion detection and interval images made processing camera data very time consuming. The time taken to store, manage, process, and analyze the camera data exceeded the time needed to conduct the live trapping study, which is contrary to previous comparisons of the methods. De Bondi et al. (2010) found camera trapping to be more time and cost effective; however, their study only assessed presence and had low camera effort $(n=5)$. Torre et al. (2005) also found camera trapping to be a more time effective means of estimating wood mice (Apodemus sylvaticus) relative abundance in different habitats. Although their study had a higher effort, their cameras produced far smaller volumes of images due to the limitations of film-based camera traps. Much of the time required to process camera data during my study was spent reviewing thousands of additional images looking for small disturbances in the soil that were used in the miss analysis. Development of the motion detection software aided in reducing the number of images that needed to be reviewed. Unfortunately, the software also required considerable time investment to develop and would have been more effective if missed images were not taken into consideration.

Recording interval images produced two benefits for the additional time cost. These images captured records of ectothermic animals, such as western toad, that would have been missed otherwise. While these data are of little use to the current study, it is proof of concept that this method could be utilized to study other ectotherms. Thus, new 
avenues of research for studying endangered amphibian populations around the world could be explored using this method. The largest benefit of the interval images was that it provided evidence of missed captures, which can affect capture rate indices. This result also provided field data supporting previous laboratory studies that showed decreased motion sensitivities in cameras at higher ambient temperatures (Swann et al., 2004). The missed image analysis also demonstrated that Reconyx HC450 units missed one in four endothermic encounters on average and that the miss rate was not significantly different across individual camera units. Missed images quantified using this method could be used to assess the sensitivity of different camera trap models in field situations independent of one another, potentially even while researching other topics. Previous attempts to field test camera sensitivities have done so by placing cameras side-by-side (Kelly \& Holub, 2008; Damm et al., 2010; Hughson et al., 2010). While this method provides relative capture rate differences, it cannot estimate absolute miss rates. An extension of the miss analysis is also underway. Scrutiny of the tracks left in the soil after recorded capture events revealed that species and gait information is recorded in the disturbance patterns. Analysis of similar gait patterns seen in missed events could lead to identification of the missed species. If species information could be reliably obtained from missed images, it could improve density estimates from all camera trap sampling methods. Not surprisingly, the gait patterns analyzed so far suggest that movement speed may be one of the major determinants for why camera traps fail to capture some events and that accurate identification of gait patterns is dependent on substrate type. Further 
development on how to incorporate species information from missed captures into a formal statistical model is still in progress.

While this study used a different methodology to find the most appropriate hit window, the results are similar to the optimal 90 min hit window estimated for North American deer mice and Northern red-backed voles (Myodes rutilus) by Villette (2014). The same study found larger animals to have a smaller, 5 min, optimal hit window. Why the larger hit window works so well for small mammals is unclear and requires further research into hit windows across other taxa. However, one notable difference between these species is the use of burrows. Voles, deer mice, and kangaroo rats all utilize burrows, whereas the larger animals studied did not. It is possible that the movement habits around burrows produce overdispersed capture data and thus require longer hit windows.

The positive relationship between live trapping density and camera capture rates supports previous research that camera capture rates are an index of density (Carbone et al., 2001; Rowcliffe et al., 2008; Villette, 2014). The linear model derived here may be useful to others studying medium-sized kangaroo rats, but its applicability is questionable as few studies have evaluated this method across populations (Carbone, 2001). The regression also showed that there is a relationship between ambient temperatures and miss rates, which should be included as a covariate to capture rate in future research using capture rate indices. Especially since data on ambient temperatures are easily obtainable from public weather records or from metadata stored in camera images. 
The lack of a significant relationship between live trapping density estimates and even the best SC model density estimates showed that the SC model did not perform well during much of this study. However, the changing shape of the camera array and low sample sizes made overarching conclusions difficult. Despite not producing a significant model with LT density, the SC model retains value as it is the only model that estimates density from camera data alone and fully maintains the non-invasive benefits of camera trapping. Furthermore, some sessions produced reasonable parameter estimates using the SC model, and it is worthwhile to assess why some sessions succeeded while others failed. During successful sessions, four traits were observed that may account for their success: 1) the width of the camera grid, 2) the number of cameras with zero captures, 3) the abundance-to-camera ratio, and 4) movement assumptions that fit the observed data.

Density estimation may have been negatively affected by the highly elongated shape of the trapping grid during sessions S1 and S2. The area of the buffer strip added during sessions S1 and S2 was much larger relative to the area of the trapping grid than in other sessions. This reduced the relative information content of the state-space and likely hindered the parameter estimation process. Estimation of home range centers from spatial autocorrelation was difficult when the grid was only 1-2 cameras wide, which further contributed to poor abundance estimates. To insure more accurate estimates, future studies should minimize the area of the buffer strip relative to the trapping grid area and establish trapping grids that span multiple home ranges.

Density plots of home range centers (Appendix E) highlight the importance of cameras with zero captures. Mathematically, the zeros represent gaps where home range 
centers are unlikely given the data, whereas biologically, these zeros represent gaps between individual home ranges. These gaps provide better evidence of spatial autocorrelation and make distinct individual home ranges more apparent. In situations where home ranges overlap, the capture data often become too noisy to differentiate individual home ranges, which causes estimates of abundance to decrease in accuracy. Species with highly territorial and evenly spaced home ranges are likely best suited for this model. For non-uniquely identifiable species with overlapping home ranges, using the calibrated capture rate indices (Carbone et al., 2001; Villette, 2014) or the REM (Rowcliffe et al., 2008) may be more appropriate as these methods rely on more uniform distributions of animal movement. The converse may also be true, methods dependent on overall capture rate may fail if points are too autocorrelated. In these situations, the SC model would work best. Successful SC model sessions had good convergence of home range centers. This convergence may be a good indicator of overall model fit when using the SC model. Whether or not capture data can pass one of the various tests from geospatial statistics for assessing spatial autocorrelation may also be an option for assessing the applicability of the SC model to a given dataset.

The precision of $\mathrm{CT}$ session abundance estimates increased as the camera-toabundance ratio increased. During sessions S1 and S2, the camera-to-abundance ratio was too small and abundance estimates declined. Conversely, sessions S5-S8 had a higher camera-to-abundance ratio and produced abundance estimates with better accuracy and precision. Unfortunately, this pattern did not hold when the number of cameras was effectively doubled in the CTMix sessions. Here, the ranging patterns of 
individuals had so much detail that it interfered with the SC model's assumption of circular home ranges. Thus, the optimal camera-to-abundance ratio is likely to be intermediate. Too many cameras and distinctly non-circular ranges inhibit model fit; too few cameras and there is a lack of spatial autocorrelation. I estimate that two to four cameras are needed to create a sufficient grouping of captures to estimate an individual's home range center, and two to four additional zeros may be required around the home range to separate it from other home ranges. Considering this, the camera effort apparently needed to obtain reasonable estimates of abundance using the SC model may be quite large. In Chandler and Royle's (2013) initial study a $15 \times 7$ array of avian point counts was used as the capture data. Due to processing time, data storage, and initial camera cost, an equivalent effort using camera traps over any appreciable amount of time may be impractical for most researchers. Applying the dual placement method used in the CTMix datasets may be an alternative; however, that method halves the sample size of the capture data. To account for limited capture data, researchers can extend the session period, but this may interfere with the assumption of population closure.

Possibly the most important factor in the SC model's success was whether or not the movements of the kangaroo rats matched the model's assumptions. Perhaps the best example of this was the low CT-specific Bayesian p-values in the CT-LT and CTMx-LT datasets. This lack of fit was a direct result of the capture rates observed in the camera trap data not aligning with the capture rates expected by the model. Similar issues with lower model fit were also seen in the CT and CTMix implementations with a prior on $\sigma$, albeit to a lesser extent. Paradoxically, Bayesian p-values from the CT and CTMix 
sessions without a prior on $\sigma$ typically had Bayesian p-values around 0.5 , incorrectly suggesting that the implementations with the most inaccurate abundance estimates fit best. The lack of fit with the movement model caused the MCMC estimation process to explore parameter estimates with higher likelihood values. Since there is no impact on model fit for estimating higher abundances, parameter estimates with many small home ranges can perfectly describe the capture history and obtain high fit statistics. This behavior in the MCMC estimation process can likely explain any dataset that does not sufficiently meet the movement model's assumptions, potentially producing seriously misleading results. During CT-LT sessions S4, S5, and S9, notably high $\sigma$ estimates were produced. This anomaly may also be a result of poor model fit. Here, overly-large $\sigma$ values were produced and allowed single home ranges to encompass the entire study site, which resulted in a nearly uniform distribution of capture rates across the state-space. Problems with movement model fit also appear in other studies which have used the SC model. Ramsey et al.'s study (2015) reported overly-small estimates of $\sigma$, and Chandler and Royle (2013) reported using a prior on $\sigma$ to inhibit the SC model from estimating overly-large estimates of $\sigma$. In light of the findings produced here, the density values reported in both the Ramsey et al. (2015) study and the Chandler and Royle (2013) study may have been biased by inaccurate home range size estimates. However, neither study compared the SC model results against independent estimates of density, so firm conclusions are difficult. However, the study conducted here sampled very few individuals, and the effects of poor model fit may have been mitigated if more individuals had been sampled. 
Placing a prior on $\sigma$ improved model accuracy and precision in almost every CT and CTMix session by constraining the parameter space of the model to biologically plausible values of $\sigma$. However, the use of a prior also makes density estimates highly dependent on the choice of prior parameters. Unfortunately, this puts researchers in a difficult position, as the SC model may require the application of a prior on $\sigma$ to be effective. At the very least, a defined minimum bound on $\sigma$ may be required so values smaller than the minimum trap spacing are not possible during MCMC estimation. One option for implementing a minimum bound on $\sigma$ is using the normal cumulative density function as an improper prior. Under this formulation, the mean and standard deviation would be calculated from the actual minimum trap spacing. This would require trap spacing to be carefully chosen ahead of time to insure the study species' home ranges cannot fall between camera stations.

While both the EP and HN functions produced similar results, they differed in how they fit model parameters. Mean $\lambda_{0}$ estimates were two times larger with the EP detection than with the $\mathrm{HN}$ function. Figure 13, shows comparisons of the two detection functions graphed using the estimated modal parameter values from the CT session posteriors. The middle of these functions often aligned with the observed trapping histories, but the sections at zero and past $\sigma$ differed greatly. Two possible reasons explain why the EP function had slightly better fit and estimates of abundance. One reason is that the thicker tail of the EP function accounts for occasional captures far from the home range center. For this reason, the EP function, or even $a$ values less than 1, may be preferred in situations where animal movements do not fit model assumptions well. 


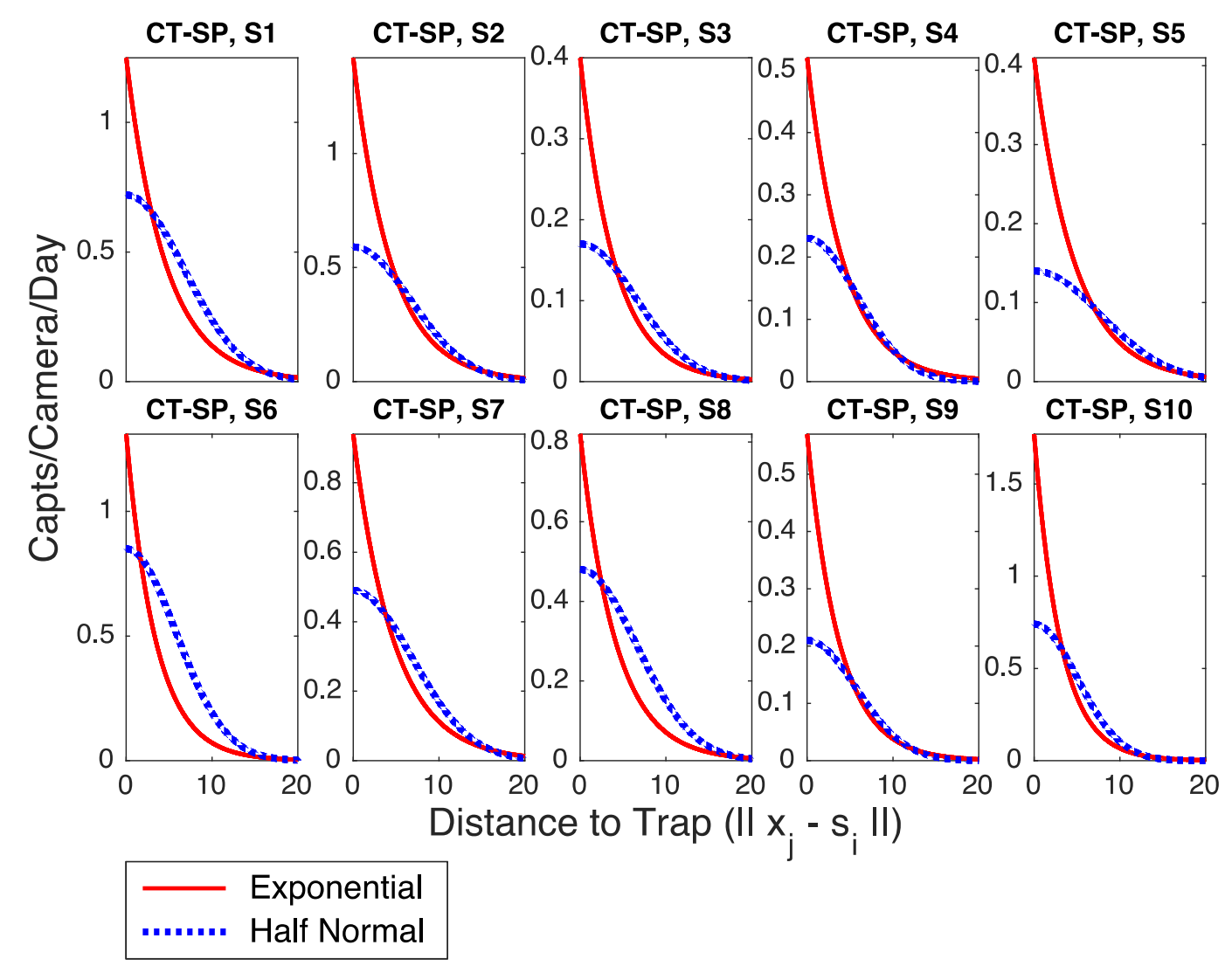

Figure 13. Comparisons of the $\mathrm{EP}$ and $\mathrm{HN}$ detection functions graphed using modal posterior estimates from $\lambda_{0}$ and $\sigma$. The Y-axis represents the estimated camera capture rate. The $\mathrm{X}$-axis represents the distance from a given home range center, $s_{i}$, to a given trap, $x_{j}$ (i.e., $\left.\left\|x_{j}-s_{i}\right\|\right)$. Note the alignment of the two functions in their middle sections and the differences in their peaks.

The second possible reason for the better fit was that the EP function best describes the movement pattern of kangaroo rats. Several radio telemetry and live trapping studies show that many species of kangaroo rat utilize a core area of their home range substantially more than outlying areas (Fitch, 1948; Schroeder, 1979; Behrends et al., 1986; Shier \& Randall, 2004; Cooper \& Randall, 2007; Meshriy et al., 2011). Shier and 
Randall's (2004) work on Heermann's kangaroo rats showed that females exhibit this pattern of range use but males did not, which may explain the more favorable results when no males were present. Additionally, Schroeder's (1979) study on the banner-tailed kangaroo rat (D. spectabilis) produced range use patterns that closely matched the shape of the EP function.

The CT-LT home range center predictions placed a narrow-faced kangaroo rat, $\mathrm{T} \# 614$, in the middle of the canyon during sessions S3 and S4 (Appendix D). This species is thought to prefer the inner portions of chamise chaparral (Hawbecker, 1940; Best, 1992), which is contrary to where the home range center was placed in the CT-LT dataset. This likely occurred due captures in the camera trap data that were unmatched in the live trapping data. The camera trap captures were likely from T\#611, a Heermann's kangaroo rat captured during LT session S3 but not S4. T\#611 was likely present through much of CT session S4 and generated the observed CT capture history. Since T\#611 was not captured during live trapping session S4, the CT-LT estimation process incorrectly placed T\#614's home range center on the canyon floor to explain the camera trap captures. This inconsistency is likely the result of unlucky timing of the LT trapping sessions or possibly trap avoidance by $\mathrm{T} \# 611$.

\section{Suggestions for Future Use of the Spatial Capture Model}

The SC model shows potential for density estimation, but several issues need to be resolved before it can be implemented with confidence. The most significant issue is the error that occurs when a study species' movement patterns do not match the model's assumptions. Several factors that influence this could be incorporated into the model to 
alleviate the problem including: the area covered by cameras, animal resource selection, study season selection, and non-invasive range use information such as burrow surveys.

During this study, cameras were placed at approximately the same height and angle, with moderate variation. This variation may account for some heterogeneity in capture rate but was believed to be minimal. REM density estimates (Rowcliffe et al., 2008) incorporate the area covered by cameras into its estimates of density assuming that a wider field of view produces more captures (Rowcliffe et al., 2011). The variation in area covered by different camera traps could be incorporated as a covariate in the SC model and may improve precision. However, as the distance between the camera and animal increases detectability likely decreases (Rowcliffe et al., 2011). The optimal area covered by a camera is then a balance between rate of capture and the rate of missed images. Finding this optimal distance may be an important step in optimizing future studies that cannot logistically implement a larger camera effort. The missed image analysis described here could aid in finding this balance before a study begins.

Species-specific and individual-specific microhabitat preferences can have a large effect on SC model success. Integrating resource selection data (Royle et al., 2013) into the camera trap study could alleviate some of the issues caused by heterogeneous range use. This method was not applied to the current study since the limited number of individuals sampled would not have produced reliable resource selection data. Studies using resource selection functions should consider an initial study that surveys a wide range of habitats and a large number of individuals in order to derive selection function parameters. The resulting data could then be used to estimate covariates of capture rate in 
the SC model. These covariates would likely reduce heterogeneity in camera capture rates and insure better movement model fit.

Some species may have movement patterns that fit the movement model better during particular seasons, such as breeding seasons. In these cases, it may be warranted to limit sampling periods to a breeding or non-breeding season where the range use pattern of a species best matches the model. The success of the SC model during sessions S5, S6, and S7 may have been due in part to seasonal differences in movement (Cooper \& Randall, 2007); though the female-only population made inference difficult as females may maintain minimal home ranges regardless of season (Randall, 1991; Perri \& Randall, 1999; Shier \& Randall, 2004; Cooper \& Randall, 2007). Some species can have consistent home range sizes regardless of densities (Perri \& Randall, 1999). Such species represent a problem for the SC model in terms of conservation monitoring. This is because lower-value, low-density populations with widely spaced home ranges may be easier to obtain reliable density estimates from than higher-value, high-density populations.

Review of the MCMC's step-by-step estimates of home range centers showed that home range centers were frequently placed directly atop one another. While it is important to maintain this possibility for general use of the model, a model formulation that includes territoriality (Reich \& Gardner, 2014) would likely improve estimates when species are distinctly territorial. The level of territoriality can be estimated from sources such as live trapping or published literature, and then used as a prior in the SC model to improve precision by limiting the estimates of overlapping home range. 
Another option for increasing model accuracy is to incorporate knowledge through other survey methods. For instance, problems resulting from overlapping home ranges could be alleviated through the incorporation of burrow survey data. An informal burrow survey was conducted during S7 that covered the home ranges of T\#615 and T\#626. The locations of burrows most likely to be inhabited by active kangaroo rats aligned reasonably well with the home range center estimates from the CT-LT data. Similar to using radio tracking data to improve estimation of home range radius in some SMR studies (Sollmann et al., 2013a \& 2013b), burrow locations could be used as an informative prior to better estimate home range center locations. These data could be combined with a camera survey of burrow occupancy to determine which species are using certain burrows, further improving precision.

The SC model may be most useful by implementing it with other methods. In this study, the CT-LT data gave a more complete picture of home range usage. Radio tracking studies have suggested that live trapping data may overestimate range size if individuals are caught far from their home burrows (Schroeder, 1979). During this study the opposite was true; home range sizes estimated from LT dataset were much smaller than those from the CT-LT dataset. In many radio tracking studies researchers approach kangaroo rats to within a few meters on foot but provide little discussion as to how this means of approach affects kangaroo rat behavior (Behrends et al., 1986; Perri \& Randall, 1999; Shier \& Randall, 2004; Cooper \& Randall, 2007). During these encounters, kangaroo rats may retreat to the safer, inner portions of their range, which could negatively bias home range size estimates. Nevertheless, a radio tracking study by Schroeder (1979) that used 
triangulation instead of handheld antennas found results similar to previous radio tracking studies. However, the Schroeder study focused on the highly territorial banner-tailed kangaroo rat, which makes differences between the two methods difficult to discern given the available data. Using the CT-LT method to study range use may be a lessinvasive alternative to radio tracking as it has less of an observer effect, but direct comparisons are needed to probe this question further. The CT-LT method could be further improved by using individual marks visible to the camera traps. The top-down angle used here made it nearly impossible to view the ear tags, let alone the inscribed numbers. Techniques which use unique markers viewable to camera traps would solidify range use information derived from the CT-LT method, but placing such unique markers on the animals would increase the invasiveness of the study.

While decreasing the number of individuals sampled during this study aided in examining questions about camera effort when using the SC model, it also emphasized the importance of sample size as it applies to parameter estimation. When sample size was low, models often produced strange and misleading results. It is important to take all parameter values into account when evaluating estimates and assess how they function both in the model and in the species. To do so requires researchers to understand the underlying models, which can be time consuming and difficult if one is not familiar with the underlying concepts. Calibrating indices and using models such as the REM have an advantage in this regard, as they are much simpler to understand and implement. While this study has shown promise for using camera capture rates as indices of density, little information exists on whether or not such indices are comparable across different 
populations of a given species. Thus, unless information on daily movement can be incorporated, their usefulness may not extend beyond site-specific monitoring purposes (Rowcliffe et al., 2008; Rovero \& Marshall, 2008; Cole, 2013; Villette, 2014). While published information on home range sizes and daily movements can be easily incorporated into both the SC model and the REM, information on home range size is likely much easier to obtain (Rowcliffe et al., 2012).

Some species are likely more suited to the SC model than others. Breeding passerines, like those studied by Chanlder and Royle (2013), may be an optimal species choice for the SC model as they are distinct central place foragers, and range use by flying animals is less restricted by plant coverage. On the other hand, kangaroo rats utilize heterogeneously placed surface runways for travel between foraging sites (Hawbecker, 1940; Tappe, 1941; Fitch, 1948). This pattern of movement can violate the assumptions of the SC model and can affect estimates of density. This effect is more likely to be observed in smaller, scatter-hoarding kangaroo rat species such as Merriam's kangaroo rat (D. merriami), Ord's kangaroo rat (D. ordii; Behrends et al., 1986; Perri \& Randall, 1999) and potentially the Fresno kangaroo rat (D. nitratoides). Conversely, larger, larder hoarding species such as the giant and banner-tailed kangaroo rats hold restricted and well-defended home ranges that are more likely to meet SC model assumptions (Schroeder, 1979; Cooper \& Randall, 2007). It has been suggested that medium sized kangaroo rats, such as Heermann's, exhibit range use patterns that are intermediate (Shier \& Randall, 2004), which may account for the partial success of the SC model observed during this study. 
The question still remains as to whether or not camera trapping could replace live trapping for monitoring small mammal populations. While the SC model and calibrated capture rate indices have potential for monitoring broad population trends, they produce far less information than live trapping. With live trapping, a researcher can obtain more reliable measures of density as well as information on mass, sex, age, morphology, body condition, and life span. Such information can be crucial to truly understanding the ecology of a population. Considering the benefits of live trapping, there would need to be an extraordinarily strong need for a non-invasive method in order to choose camera trapping over live trapping. Otherwise, implementation of additional safeguards against mortality and stress are likely sufficient in mitigating the invasiveness of live trapping.

The development of methods that can non-invasively sample populations of nonuniquely identifiable species is still of considerable use to the ecological community since a large portion of animals fall into this category. Techniques such as the SC model represent a potentially powerful new tool for this task but may only be viable when the spatial ecology of the species being studied matches the assumptions of the model. Consideration of these model's assumptions in conjunction with their limitations and applicability is key to further research and development within this field. 


\section{References}

Anile, S., Ragni, B., Randi, E., Mattucci, F., \& Rovero, F. (2014). Wildcat population density on the Etna volcano, Italy: A comparison of density estimation methods. Journal of Zoology, 293, 252-261.

Balderama, E., Gardner, B., \& Reich, B. (2012). Species abundance estimation methods for single-catch trap surveys. Environmetrics.

Bartolommei, P., Manzo, E., \& Cozzolino, R. (2013). Evaluation of three indirect methods for surveying European pine marten in a forested area of central Italy. Hystrix, the Italian Journal of Mammalogy, 23, 91-94.

Bayrakçi, R., Carey, A. B., \& Wilson, T. M. (2001). Current status of the western gray squirrel population in the Puget Trough, Washington. Northwest Science, 75, 333341.

Behrends, P., Daly, M., \& Wilson, M. I. (1986). Aboveground activity of Merriam's kangaroo rats (Dipodomys merriami) in relation to sex and reproduction. Behaviour, 96, 210-226.

Best, T. L. (1992). Dipodomys venustus. Mammalian Species, 403, 1-4.

Best, T. L., Chesser, R. K., McCullough, D. A., \& Baumgardner, G. D. (1996). Genic and morphometric variation in kangaroo rats, genus Dipodomys, from coastal California. Journal of Mammalogy, 77, 785-800.

Borchers, D. (2012). A non-technical overview of spatially explicit capture-recapture models. Journal of Ornithology, 152, 435-444.

Bradford, D. F. (1976). Space utilization by rodents in Adenostoma chaparral. Journal of Mammalogy, 57, 576-579.

Brylski, P. V., Collins, P. W., Pierson, E. D., Rainey, W. E., \& Kucera, T. E. (1998).

Terrestrial mammal species of special concern in California. Sacramento, California: California Department of Fish and Wildlife.

Bunty, J. (2015). Estimating tiger (Panthera tigris) prey density using camera traps and fecal accumulation rates. Retrieved from Clemson University All Theses Database. (Accession No. 2185) 
Carbone, C., Christie, S., Conforti, K., Coulson, T., Franklin, N., Ginsberg, J., et al. (2001). The use of photographic rates to estimate densities of tigers and other cryptic mammals. Animal Conservation, 4, 75-79.

Chandler, R. B., \& Royle, J. A. (2013). Spatially explicit models for inference about density in unmarked or partially marked populations. The Annals of Applied Statistics, 7, 936-954.

Church, R. L. (1969). Evaporative water loss and gross effects of water privation in the kangaroo rat, Dipodomys venustus. Journal of Mammalogy, 50, 514-523.

Cole, E. G. (2013). Estimating mammalian densities using automated videography at the firestone reserve, Costa Rica. Scripps Senior Theses. (Accession No. 299)

Congdon, J., \& Roest, A. I. (1975). Status of the endangered Morro Bay kangaroo rat. Journal of Mammalogy, 56, 679-683.

Cooper, L. D., \& Randall, J. A. (2007). Seasonal changes in home ranges of the giant kangaroo rat (Dipodomys ingens): A study of flexible social structure. Journal of Mammalogy, 88, 1000-1008.

Cutler, T. L., \& Swann, D. E. (1999). Using remote photography in wildlife ecology: A review. Wildlife Society Bulletin, 27, 571-581.

Damm, P. E., Grand, J. B., \& Barnett, S. W. (2010). Variation in detection among passive infrared triggered-cameras used in wildlife research. Paper presented at the Proceeding of the Annual Conference of the Southeast Association of Fish and Wildlife Agencies, 64. pp. 125-130.

De Bondi, N., White, J. G., Stevens, M., \& Cooke, R. (2010). A comparison of the effectiveness of camera trapping and live trapping for sampling terrestrial smallmammal communities. Wildlife Research, 37, 456-465.

Delehanty, B., \& Boonstra, R. (2009). Impact of live trapping on stress profiles of Richardson's ground squirrel (Spermophilus richardsonii). General and Comparative Endocrinology, 160, 176-182.

De Sa, M. A., Zweig, C. L., Percival, H. F., Kitchens, W. M., \& Kasbohm, J. W. (2012). Comparison of small-mammal sampling techniques in tidal salt marshes of the central gulf coast of Florida. Southeastern Naturalist, 11, 89-100.

Dice, L. R. (1938). Some census methods for mammals. The Journal of Wildlife Management, 2, 119-130. 
Efford, M. (2004). Density estimation in live-trapping studies. Oikos, 106, 598-610.

Efford, M. G., Borchers, D. L., \& Byrom, A. E. (2009). Density estimation by spatially explicit capture-recapture: Likelihood-based methods. Modeling demographic processes in marked populations (pp. 255-269) Springer.

Fitch, H. S. (1948). Habits and economic relationships of the Tulare kangaroo rat. Journal of Mammalogy, 29, 5-35.

Fletcher, Q. E., \& Boonstra, R. (2006). Impact of live trapping on the stress response of the meadow vole (Microtus pennsylvanicus). Journal of Zoology, 270, 473-478.

Foster, R. J., \& Harmsen, B. J. (2012). A critique of density estimation from camera-trap data. The Journal of Wildlife Management, 76, 224-236.

French, A. (1993). Physiological ecology of the Heteromyidae: Economics of energy and water utilization. Biology of the Heteromyidae, 10, 509-538.

Gardner, B., Reppucci, J., Lucherini, M., \& Royle, J. A. (2010). Spatially explicit inference for open populations: Estimating demographic parameters from cameratrap studies. Ecology, 91, 3376-3383.

Gerber, B. D., \& Parmenter, R. R. (2015). Spatial capture-recapture model performance with known small-mammal densities. Ecological Applications, 25, 695-705.

Glen, A. S., Cockburn, S., Nichols, M., Ekanayake, J., \& Warburton, B. (2013). Optimising camera traps for monitoring small mammals. PloS One, 8(6), e67940.

Goldingay, R. L., Kelly, P. A., \& Williams, D. F. (1997). The kangaroo rats of California: Endemism and conservation of keystone species. Pacific Conservation Biology, 3, 47-60.

Grinnell, J. (1922). A geographical study of the kangaroo rats of California. University of California Press.

Harmsen, B. J., Foster, R. J., \& Doncaster, C. P. (2011). Heterogeneous capture rates in low density populations and consequences for capture-recapture analysis of cameratrap data. Population Ecology, 53, 253-259.

Harvey, P. (2015). Exiftool (9.63rd ed.)

Hawbecker, A. C. (1940). The burrowing and feeding habits of Dipodomys venustus. Journal of Mammalogy, 21, 388-396. 
Hughson, D. L., Darby, N. W., \& Dungan, J. D. (2010). Comparison of motion-activated cameras for wildlife investigations. California Fish and Game, 96(2), 101-109.

Jennelle, C. S., Runge, M. C., \& MacKenzie, D. I. (2002). The use of photographic rates to estimate densities of tigers and other cryptic mammals: A comment on misleading conclusions. Animal Conservation, 5(2), 119-120.

Kane, M. D., Morin, D. J., \& Kelly, M. J. (2015). Potential for camera-traps and spatial mark-resight models to improve monitoring of the critically endangered West African lion (Panthera leo). Biodiversity and Conservation, 24, 3527-3541.

Karanth, K. U. (1995). Estimating tiger, Panthera tigris, populations from camera-trap data using capture-recapture models. Biological Conservation, 71, 333-338.

Karanth, K. U., \& Nichols, J. D. (1998). Estimation of tiger densities in India using photographic captures and recaptures. Ecology, 79, 2852-2862.

Kays, R. W., \& Wilson, D. E. (2009). Mammals of North America. Princeton University Press, New Jersey.

Kelly, M. J., \& Holub, E. L. (2008). Camera trapping of carnivores: Trap success among camera types and across species, and habitat selection by species, on salt pond mountain, Giles county, Virginia. Northeastern Naturalist, 15, 249-262.

Kelt, D. A. (1988). Dipodomys heermanni. Mammalian Species, 232, 1-7.

Krebs, C. J., Boonstra, R., Gilbert, S., Reid, D., Kenney, A. J., \& Hofer, E. J. (2011). Density estimation for small mammals from live trapping grids: Rodents in northern Canada. Journal of Mammalogy, 92, 974-981.

Kucera, T. E., \& Barrett, R. H. (2011). A history of camera trapping. Camera traps in animal ecology (pp. 9-26) Springer.

Lucas, T. C., Moorcroft, E. A., Freeman, R., Rowcliffe, J. M., \& Jones, K. E. (2015). A generalised random encounter model for estimating animal density with remote sensor data. Methods in Ecology and Evolution, 6, 500-509.

Manzo, E., Bartolommei, P., Rowcliffe, J. M., \& Cozzolino, R. (2012). Estimation of population density of European pine marten in central Italy using camera trapping. Acta Theriologica, 57, 165-172.

McCallum, J. (2013). Changing use of camera traps in mammalian field research: Habitats, taxa and study types. Mammal Review, 43, 196-206. 
McDonald, P. J., Griffiths, A. D., Nano, C. E., Dickman, C. R., Ward, S. J., \& Luck, G. W. (2015). Landscape-scale factors determine occupancy of the critically endangered central rock-rat in arid Australia: The utility of camera trapping. Biological Conservation, 191, 93-100.

Meshriy, M. G., Randall, J. A., \& Parra, L. (2011). Kinship associations of a solitary rodent, Dipodomys ingens, at fluctuating population densities. Animal Behaviour, 82, 643-650.

Negrões, N., Sarmento, P., Cruz, J., Eira, C., Revilla, E., Fonseca, C., et al. (2010). Use of Camera-Trapping to estimate puma density and influencing factors in central brazil. The Journal of Wildlife Management, 74, 1195-1203.

Noss, A., Gardner, B., Maffei, L., Cuéllar, E., Montaño, R., Romero-Muñoz, A., et al. (2012). Comparison of density estimation methods for mammal populations with camera traps in the Kaa-Iya Del Gran Chaco landscape. Animal Conservation, 15, 527-535.

O'Brien, T. G. (2011). Abundance, density and relative abundance: A conceptual framework. Camera traps in animal ecology (pp. 71-96) Springer.

O'Connell, A. F., Nichols, J. D., \& Karanth, K. U. (2011). Camera traps in animal ecology: Methods and analyses Springer Tokyo.

Otis, D. L., Burnham, K. P., White, G. C., \& Anderson, D. R. (1978). Statistical inference from capture data on closed animal populations. Wildlife Monographs, 62, 3-135.

Pearson, O. P. (1959). A traffic survey of Microtus-Reithrodontomys runways. Journal of Mammalogy, 40, 169-180.

Pearson, O. P. (1960). Habits of Microtus californicus revealed by automatic photographic recorders. Ecological Monographs, 30, 232-250.

Perri, L. M., \& Randall, J. A. (1999). Behavioral mechanisms of coexistence in sympatric species of desert rodents, Dipodomys ordii and D. merriami. Journal of Mammalogy, $80,1297-1310$.

Phase One A/S. (2012). Phase one media pro (1.4.06604 ed.). Frederiksberg, Denmark.

Phillips, D. W. (2013). Determining microhabitat use by the gulf coast kangaroo rat (Dipodomys compactus) using motion sensitive cameras and estimating population density at their northern distribution limit, Retrieved from Texas State University Digital Collections. 
Potter, C. J. (1985). Determination of endangered status and critical habitat for the Fresno kangaroo rat. United States Department of the Interior: Fish and Wildlife Service.

R Core Team. (2015). R: A language and environment for statistical computing (3.2.2 ed.). Vienna, Austria: R Foundation for Statistical Computing.

Ramsey, D. S., Caley, P. A., \& Robley, A. (2015). Estimating population density from presence-absence data using a spatially explicit model. The Journal of Wildlife Management, 79, 491-499.

Randall, J. A. (1991). Mating strategies of a nocturnal, desert rodent (Dipodomys spectabilis). Behavioral Ecology and Sociobiology, 28, 215-220.

Recce, S. (1988). Determination of endangered status for the Stephens' kangaroo rat. United States Department of the Interior: Fish and Wildlife Service.

Recce, S. (1988). Determination of endangered status for the Tipton kangaroo rat. United State Department of the Interior: Fish and Wildlife Service.

Reich, B. J., \& Gardner, B. (2014). A spatial capture-recapture model for territorial species. Environmetrics, 25, 630-637.

Rendall, A. R., Sutherland, D. R., Cooke, R., \& White, J. (2014). Camera trapping: A contemporary approach to monitoring invasive rodents in high conservation priority ecosystems. PloS One, 9(3), e86592.

Rich, L. N., Kelly, M. J., Sollmann, R., Noss, A. J., Maffei, L., Arispe, R. L., et al. (2014). Comparing capture-recapture, mark-resight, and spatial mark-resight models for estimating puma densities via camera traps. Journal of Mammalogy, 95(2), 382391.

Rovero, F., \& Marshall, A. R. (2009). Camera trapping photographic rate as an index of density in forest ungulates. Journal of Applied Ecology, 46, 1011-1017.

Rowcliffe, J. M., Field, J., Turvey, S. T., \& Carbone, C. (2008). Estimating animal density using camera traps without the need for individual recognition. Journal of Applied Ecology, 45, 1228-1236.

Rowcliffe, J. M., Kays, R., Carbone, C., \& Jansen, P. A. (2013). Clarifying assumptions behind the estimation of animal density from camera trap rates. The Journal of Wildlife Management, 77, 876. 
Rowcliffe, M. J., Carbone, C., Jansen, P. A., Kays, R., \& Kranstauber, B. (2011). Quantifying the sensitivity of camera traps: An adapted distance sampling approach. Methods in Ecology and Evolution, 2, 464-476.

Rowcliffe, M. J., Carbone, C., Kays, R., Kranstauber, B., \& Jansen, P. A. (2012). Bias in estimating animal travel distance: The effect of sampling frequency. Methods in Ecology and Evolution, 3, 653-662.

Royle, J. A., Chandler, R. B., Sollmann, R., \& Gardner, B. (2014). Spatial capturerecapture Academic Press.

Royle, J. A., Dorazio, R. M., \& Link, W. A. (2007). Analysis of multinomial models with unknown index using data augmentation. Journal of Computational and Graphical Statistics, 16, 67-85.

Royle, J. A., Karanth, K. U., Gopalaswamy, A. M., \& Kumar, N. S. (2009). Bayesian inference in camera trapping studies for a class of spatial capture-recapture models. Ecology, 90, 3233-3244.

Royle, J. A., \& Nichols, J. D. (2003). Estimating abundance from repeated presenceabsence data or point counts. Ecology, 84, 777-790.

Royle, J. A. (2011). Hierarchical spatial Capture-Recapture models for estimating density from trapping arrays. Camera traps in animal ecology (pp. 163-190) Springer.

Rutledge, M. E., Sollmann, R., Washburn, B. E., Moorman, C. E., \& De Perno, C. S. (2015). Using novel spatial mark-resight techniques to monitor resident Canada geese in a suburban environment. Wildlife Research, 41, 447-453.

Schmidt-Nielsen, B., \& Schmidt-Nielsen, K. (1950). Pulmonary water loss in desert rodents. American Journal of Physiology, 182, 31-36.

Schmidt-Nielsen, B., Schmidt-Nielsen, K., Brokaw, A., \& Schneiderman, H. (1948). Water conservation in desert rodents. Journal of Cellular and Comparative Physiology, 32, 331-360.

Schroder, G. D. (1979). Foraging behavior and home range utilization of the banner-tail kangaroo rat (Dipodomys spectabilis). Ecology, 60, 658-665.

Shier, D. M., \& Randall, J. A. (2004). Spacing as a predictor of social organization in kangaroo rats (Dipodomys heermanni arenae). Journal of Mammalogy, 85, 10021008 . 
Sikes, R. S., \& Gannon, W. L. (2011). Guidelines of the American Society of Mammalogists for the use of wild mammals in research. Journal of Mammalogy, 92, $235-253$.

Smith, D. P. (1986). Determination of endangered status for the giant kangaroo rat. United States Department of the Interior: Fish and Wildlife Service.

Sollmann, R., Gardner, B., Chandler, R. B., Shindle, D. B., Onorato, D. P., Royle, J. A., et al. (2013). Using multiple data sources provides density estimates for endangered Florida panther. Journal of Applied Ecology, 50, 961-968.

Sollmann, R., Gardner, B., Parsons, A. W., Stocking, J. J., McClintock, B. T., Simons, T. R., et al. (2013). A spatial mark-resight model augmented with telemetry data. Ecology, 94, 553-559.

Sollmann, R., Mohamed, A., Samejima, H., \& Wilting, A. (2013). Risky business or simple solution-Relative abundance indices from camera-trapping. Biological Conservation, 159, 405-412.

Stewart, G. R., \& Roest, A. I. (1960). Distribution and habits of kangaroo rats at Morro Bay. Journal of Mammalogy, 41, 126-129.

Swann, D. E., Hass, C. C., Dalton, D. C., \& Wolf, S. A. (2004). Infrared-triggered cameras for detecting wildlife: An evaluation and review. Wildlife Society Bulletin, $32,357-365$.

Swann, D. E., Kawanishi, K., \& Palmer, J. (2011). Evaluating types and features of camera traps in ecological studies: A guide for researchers. Camera traps in animal ecology (pp. 27-43) Springer.

Tappe, D. T. (1941). Natural history of the Tulare kangaroo rat. Journal of Mammalogy, $22,117-148$.

The Mathworks. (2015b). MATLAB (8.6th ed.). Natick, Massachusetts, United States.

Torre, I., Peris, A., \& Tena, L. (2005). Estimating the relative abundance and temporal activity patterns of wood mice (Apodemus sylvaticus) by remote photography in Mediterranean post-fire habitats. Galemys, 17, 41-52.

Tracy, R. L., \& Walsberg, G. E. (2001). Developmental and acclimatory contributions to water loss in a desert rodent: Investigating the time course of adaptive change. Journal of Comparative Physiology B, 171, 669-679. 
Tracy, R. L., \& Walsberg, G. E. (2001). Intraspecific variation in water loss in a desert rodent, Dipodomys merriami. Ecology, 82, 1130-1137.

Tracy, R. L., \& Walsberg, G. E. (2002). Kangaroo rats revisited: Re-evaluating a classic case of desert survival. Oecologia, 133, 449-457.

Trolle, M., \& Kéry, M. (2003). Estimation of ocelot density in the Pantanal using capture-recapture analysis of camera-trapping data. Journal of Mammalogy, 84, 607614.

Villette, Petra Mary Anne Hobson. (2014). Estimating population densities of Peromyscus maniculatus, Clethrionomys rutilus, Lepus americanus, and Tamiasciurus hudsonicus using remote cameras in the boreal forest of Yukon Territory, Canada. University of British Columbia Theses and Dissertations.

White, G. C. (1982). Capture-recapture and removal methods for sampling closed populations Los Alamos National Laboratory.

Wilson, K. R., \& Anderson, D. R. (1985). Evaluation of two density estimators of small mammal population size. Journal of Mammalogy, 66, 13-21.

Zero, V. H., Sundaresan, S. R., O'Brien, T. G., \& Kinnaird, M. F. (2013). Monitoring an endangered savannah ungulate, Grevy's zebra Equus grevyi: Choosing a method for estimating population densities. Oryx, 47, 410-419. 


\section{Appendix A. Capture Histories}

This appendix gives the capture histories for the five datasets: LT, CT, CT-LT, CTMix and CTMx-LT. For every session of data, a graphical representation is given for the locations of the traps $\left(\boldsymbol{x}_{j}\right)$, the polygon used as the statespace $(S)$, and the capture history $\left(y_{i j}\right.$ or $\left.y_{t j}\right)$. The way in which the capture histories are presented are described in their respective sections.

In addition to the capture information, the data are plotted against satellite images obtained from imagery dated 25 August, 2013 in Google Earth (C Google Earth 2016). The images were tiled together and georeferenced, and the resulting image was down sampled and annotated with the points in MATLAB. Data are displayed in UTM Zone 10S, on the NAD 1983 Datum. 


\section{Appendix A1. LT Capture Histories}

This appendix shows the capture histories, trap locations, and state space for the 10 sessions of live trapping (LT) data. The image below serves as an example. Individuals captured are represented by their three-digit tag number and a unique colored box. The same box color is also used in the home range center maps in Appendix D. If individuals were captured at the same trap on multiple nights, the tag icons are seen as stacked (for example, see 419 in upper right). Crosses represent live trap locations $\left(x_{j}\right)$, and the outline shows the state space used in analysis $(S)$.

Underlying imagery (C) 2016 Google Maps, plotted on UTM Zone 10S.

Session S1 Capure Data

LT Data

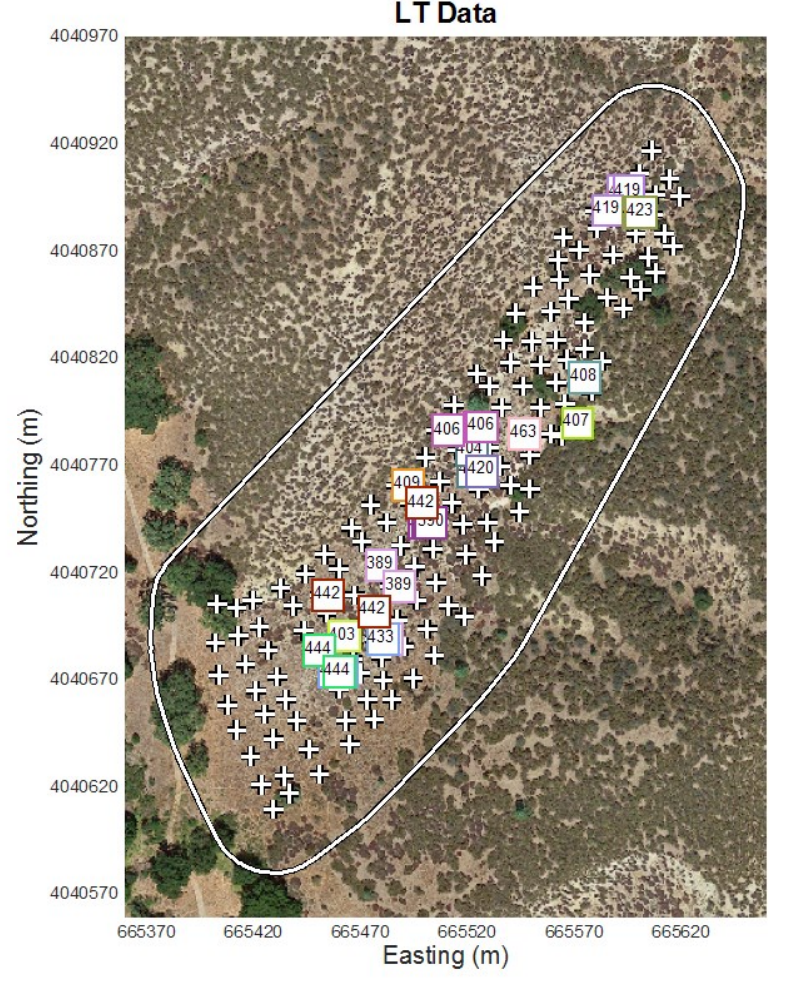




\section{Session S1 Capure Data}

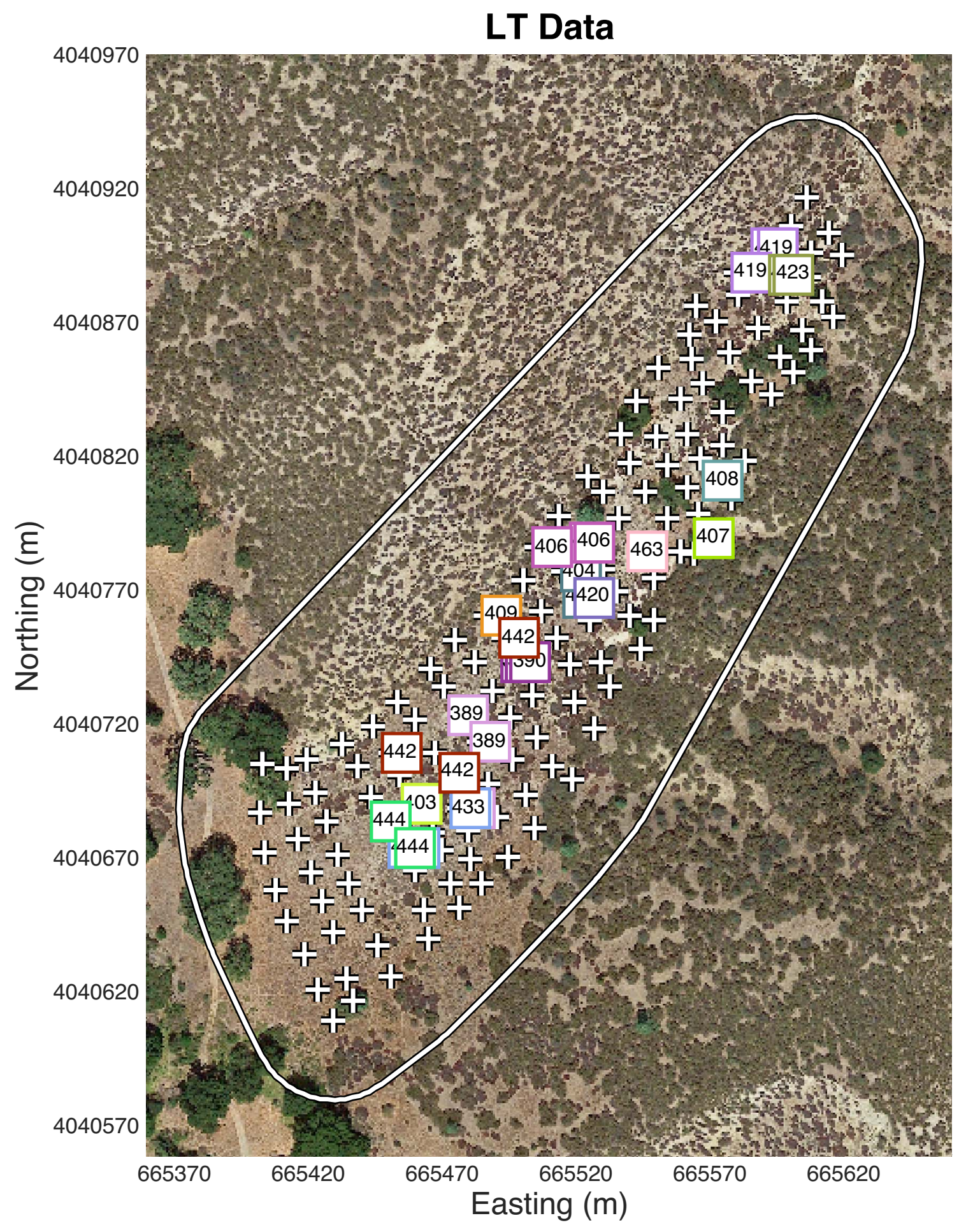




\section{Session S2 Capure Data}

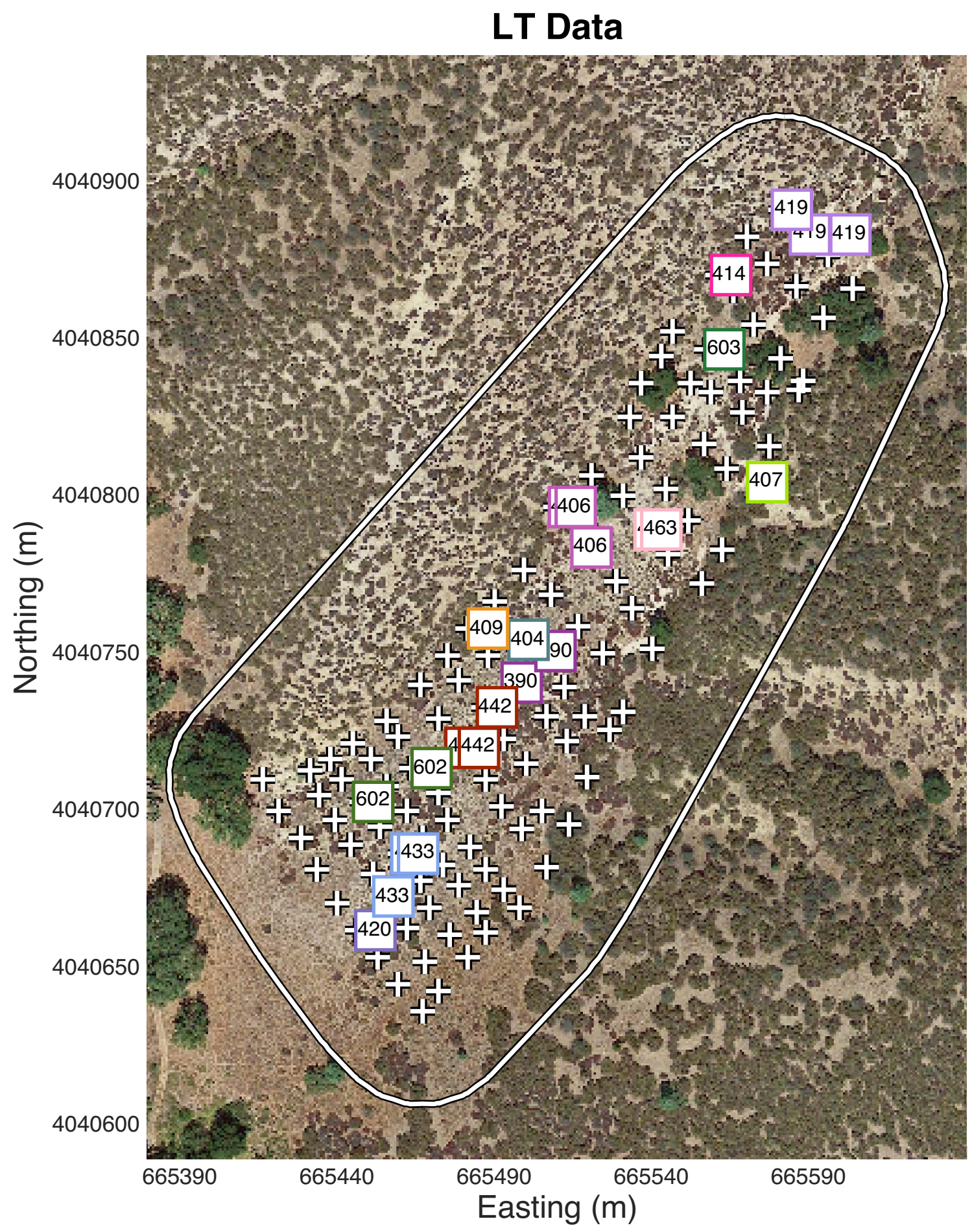




\section{Session S3 Capure Data}

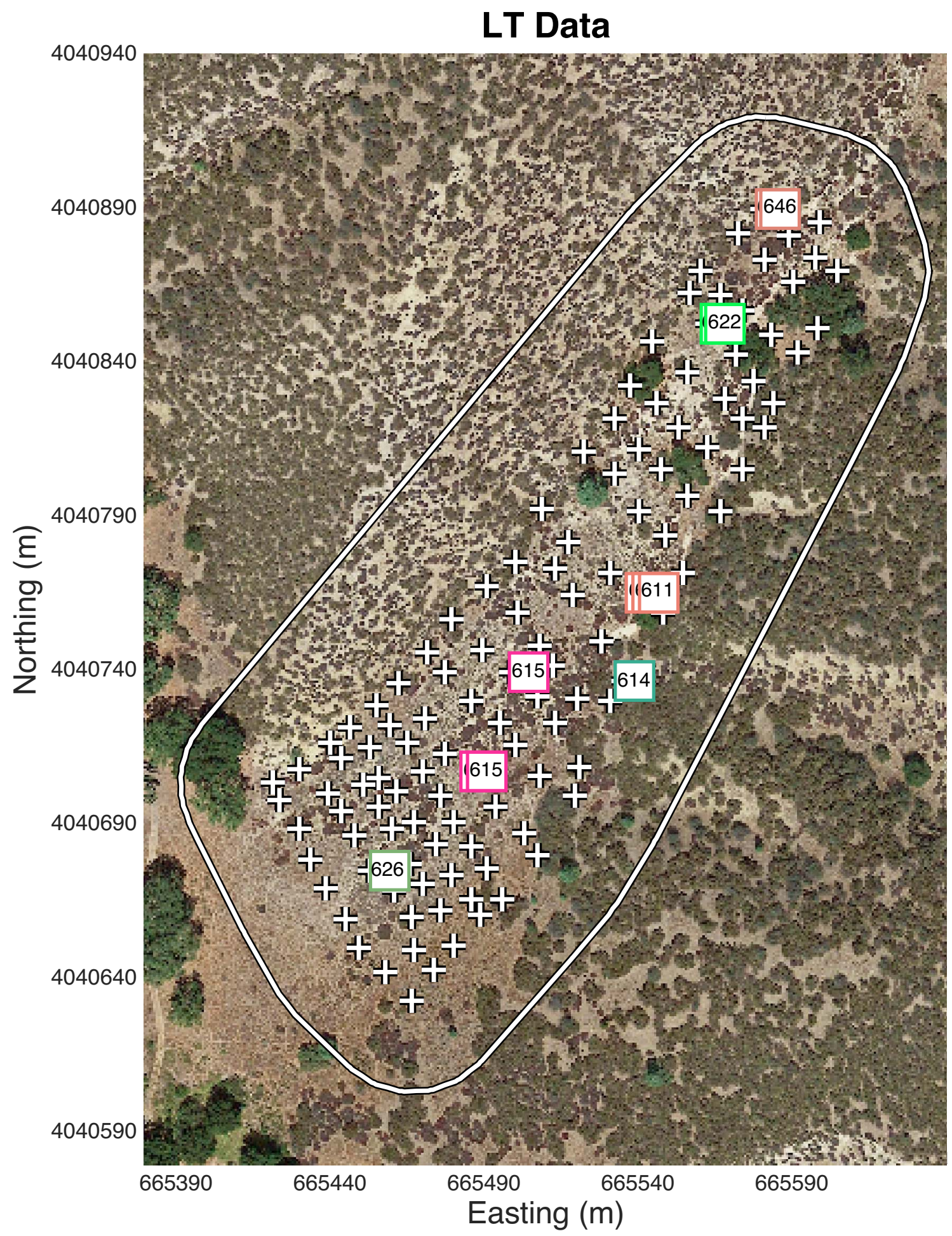




\section{Session S4 Capure Data}

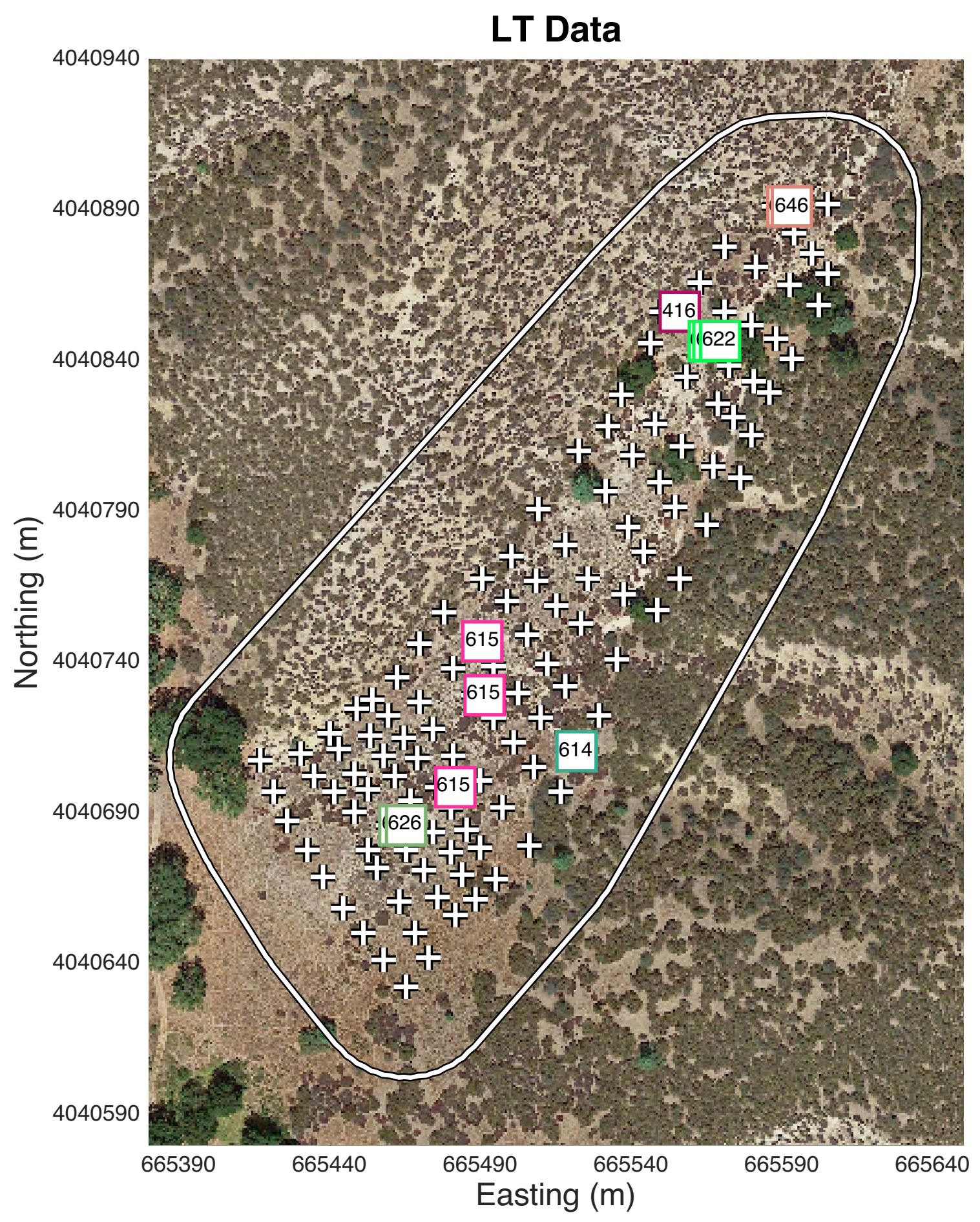




\section{Session S5 Capure Data}

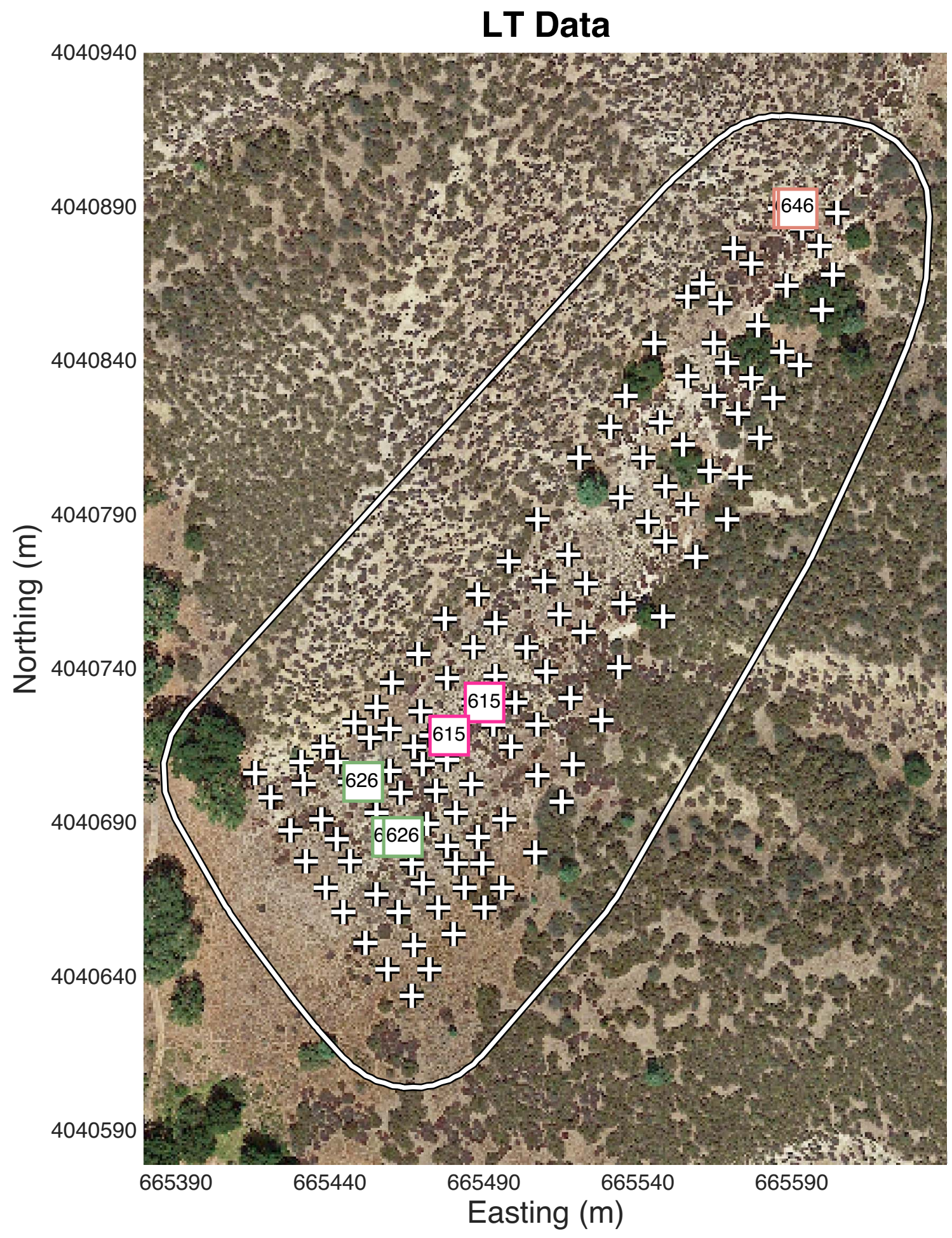




\section{Session S6 Capure Data}

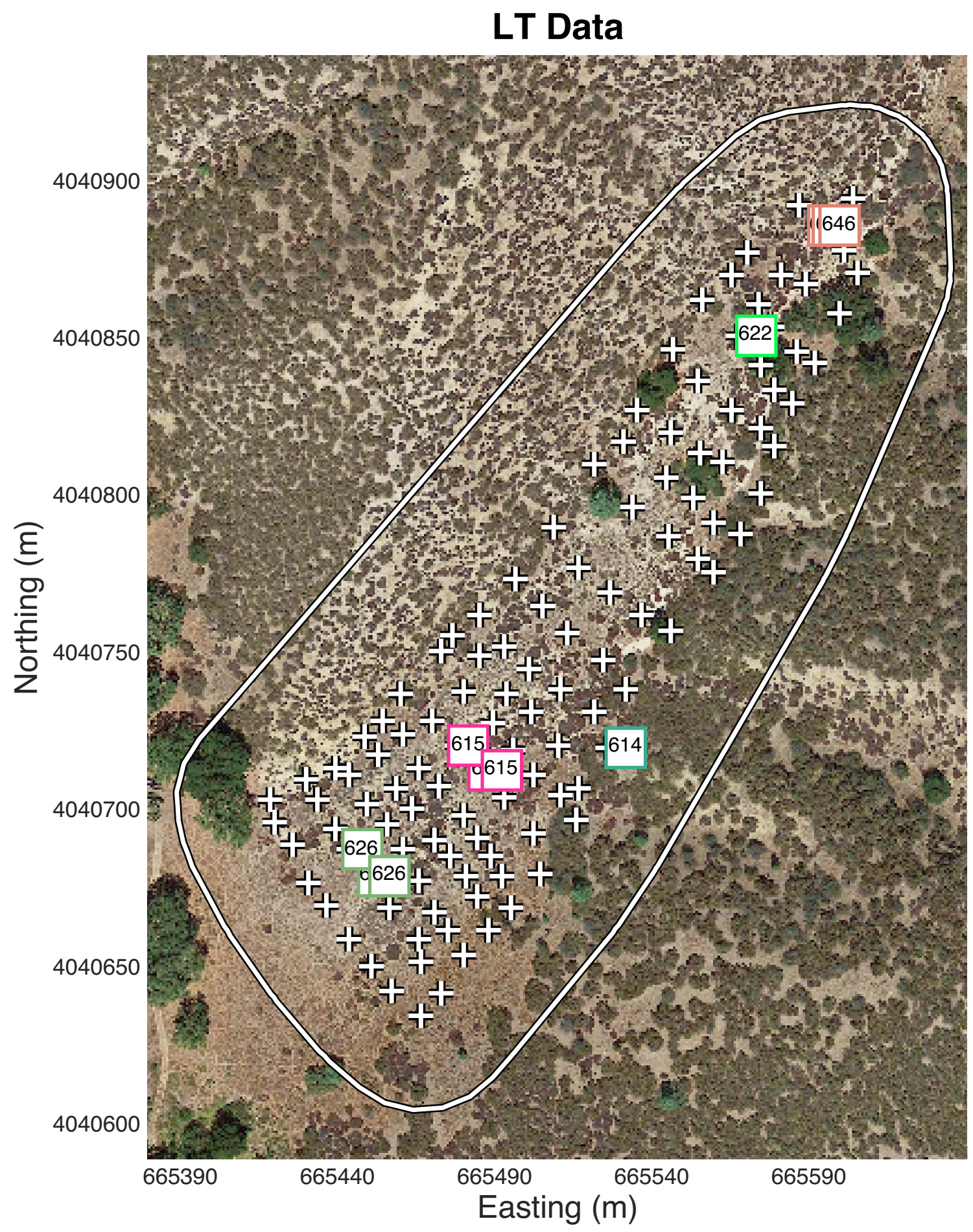




\section{Session S7 Capure Data}

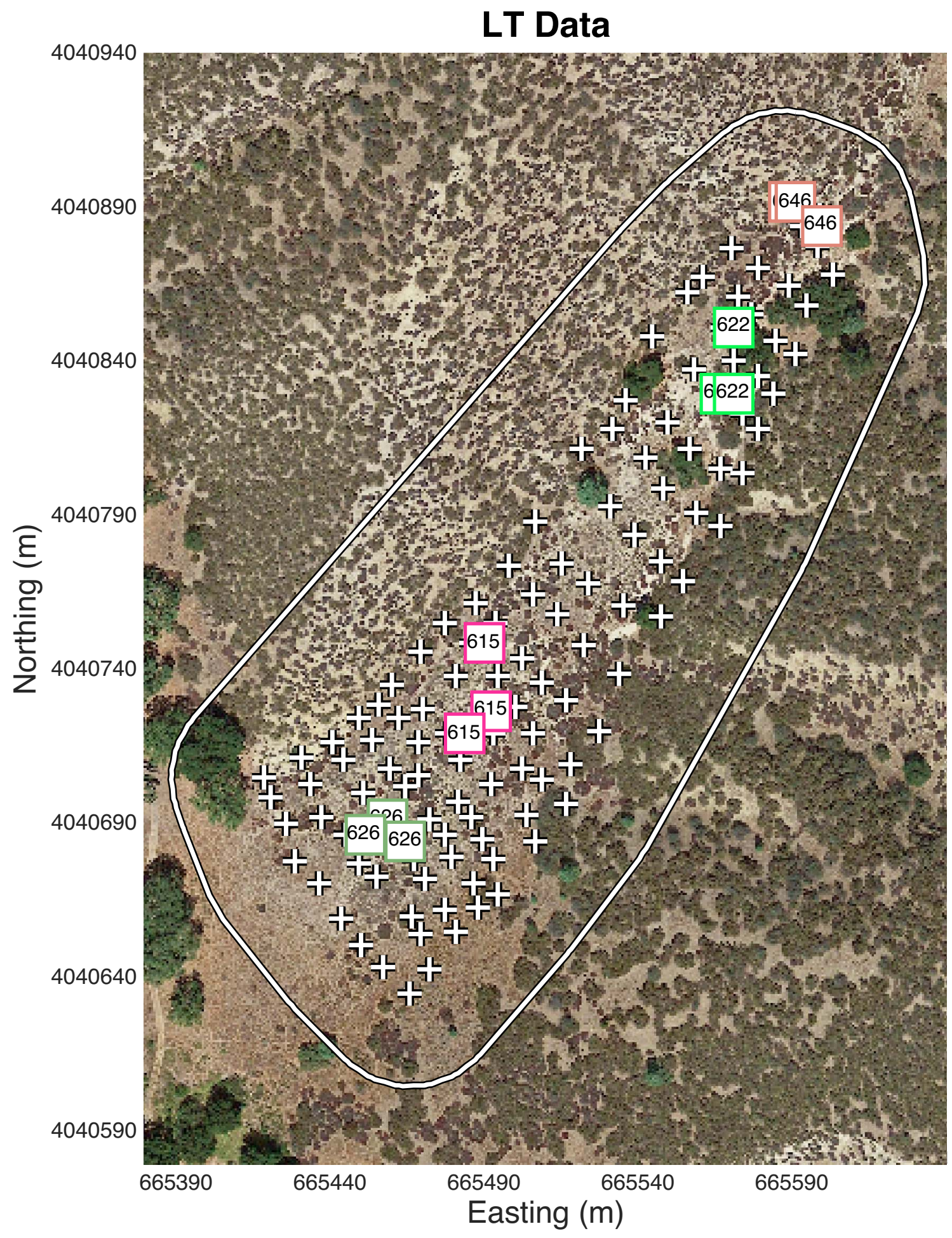




\section{Session S8 Capure Data}

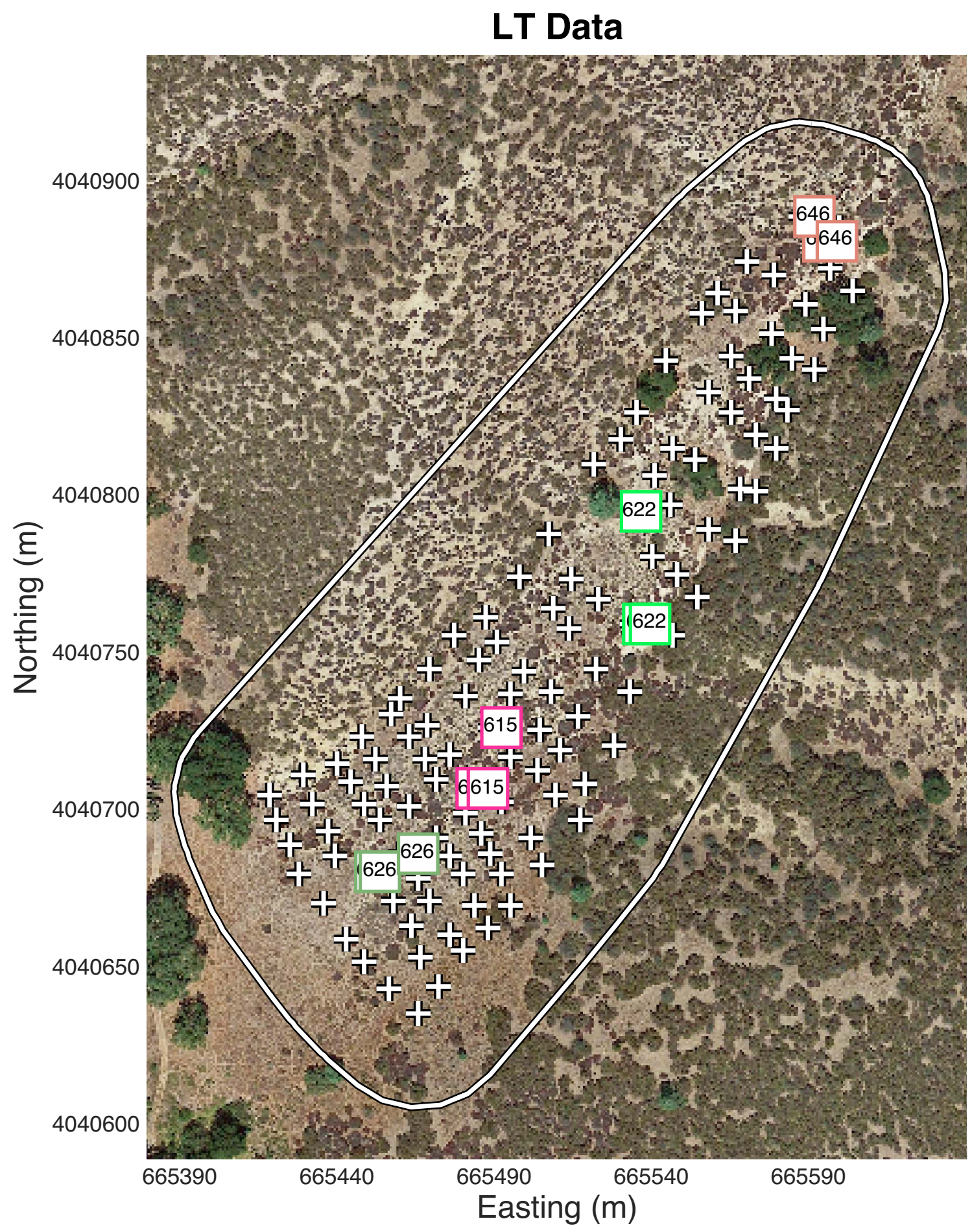




\section{Session S9 Capure Data}

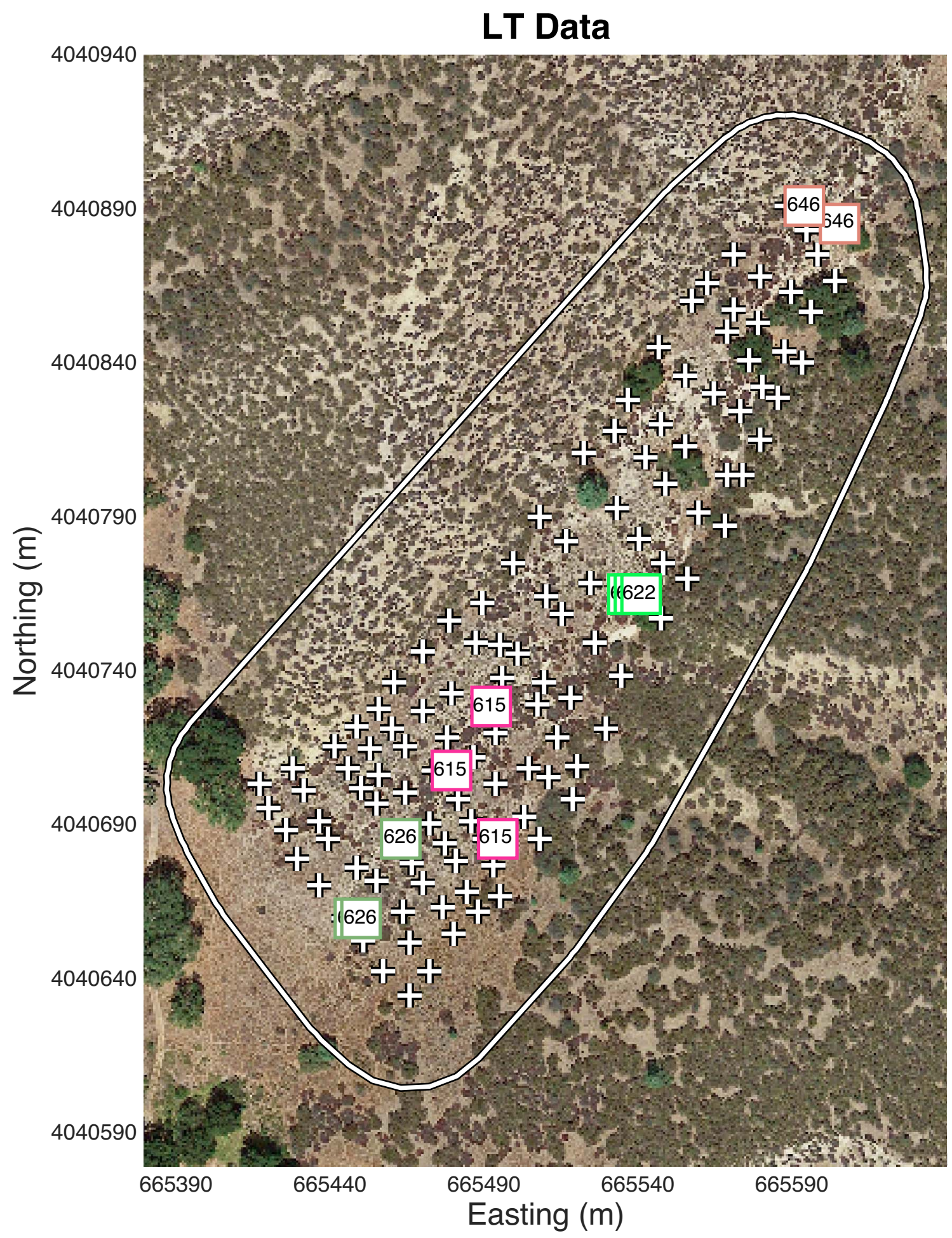




\section{Session S10 Capure Data}

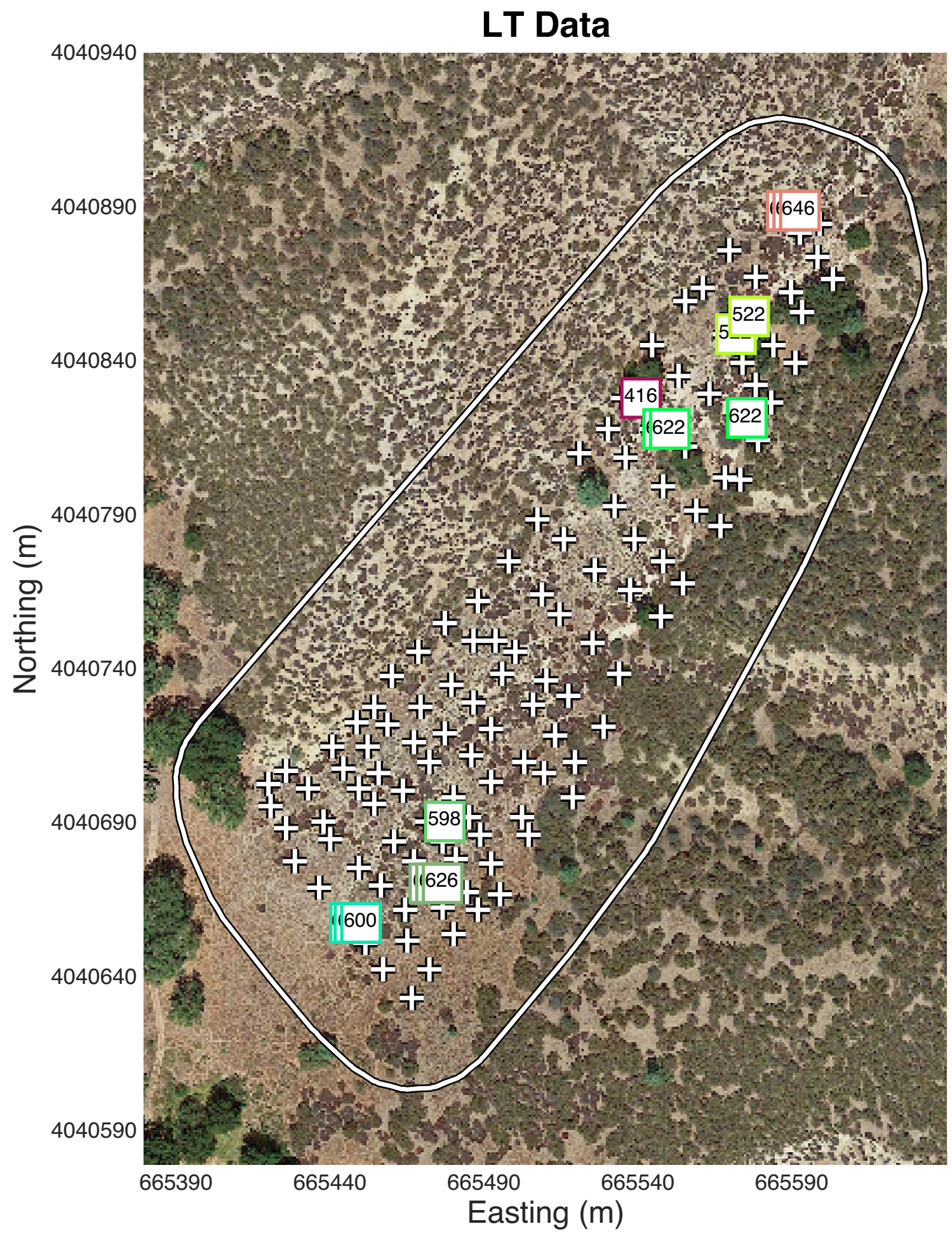




\section{Appendix A2. CT and CT-LT Capture Histories}

This appendix shows the capture histories, trap locations, and state space for the 10 sessions of camera trapping (CT) and joint trapping (CT-LT) datasets. The image below serves as an example. The CT capture histories are shown as circles of varying sizes that represent the camera's relative capture rate. Some number locations were adjusted for viewing purposes. The number listed in the center is the average number of captures per day. The CT-LT capture histories displayed here are the same as the LT capture histories but truncated to match the CT state space. In the CT-LT data, individuals captured are represented by their three-digit tag number and a unique colored box. The same box color is also used in the home range center maps in Appendix D. If individuals were captured at the same trap on multiple nights, the tag icons are seen as stacked (for example, see 419 in upper right). Crosses represent all live trap locations $\left(x_{j}\right)$, and the outline shows the state space used in analysis $(S)$.

Underlying imagery (C) 2016 Google Maps, plotted on UTM Zone 10S

Session S1 Capture Data

CT Data

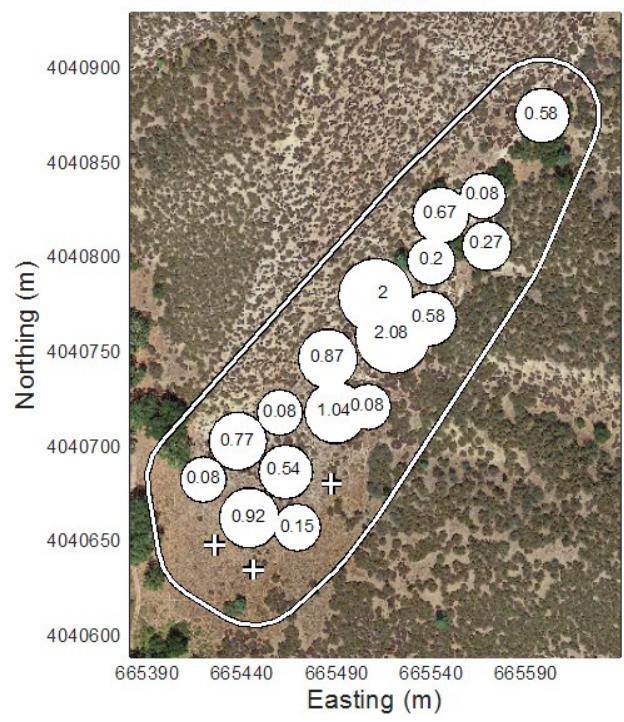

CT-LT Data

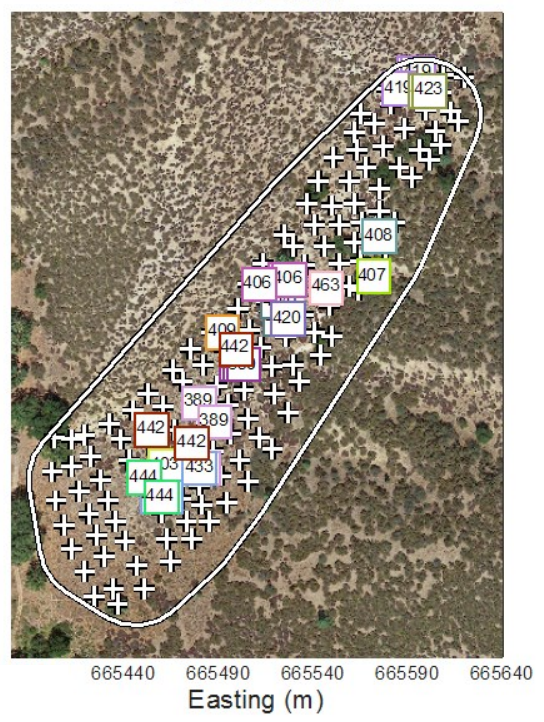




\section{Session S1 Capture Data}
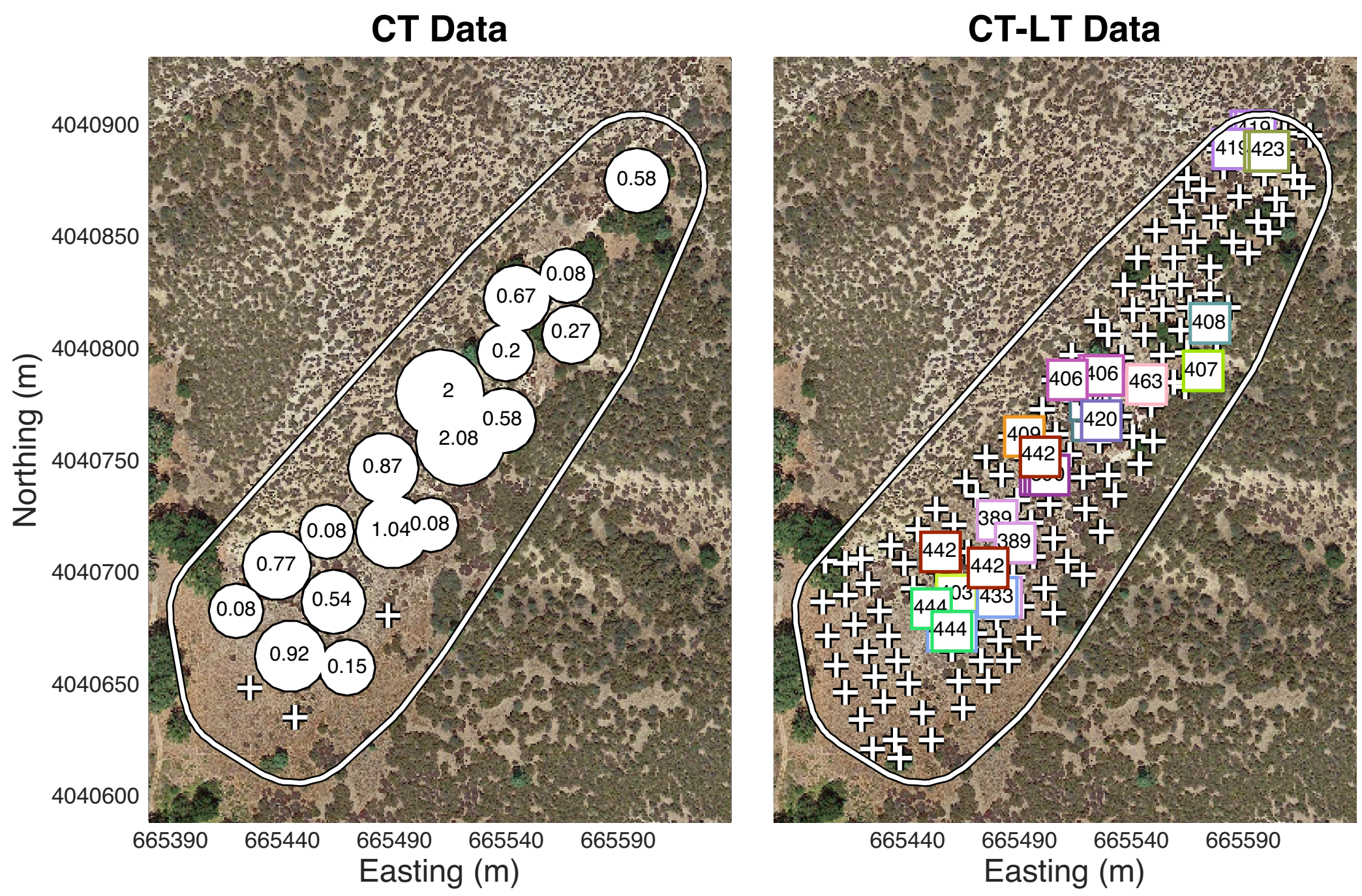


\section{Session S2 Capture Data}

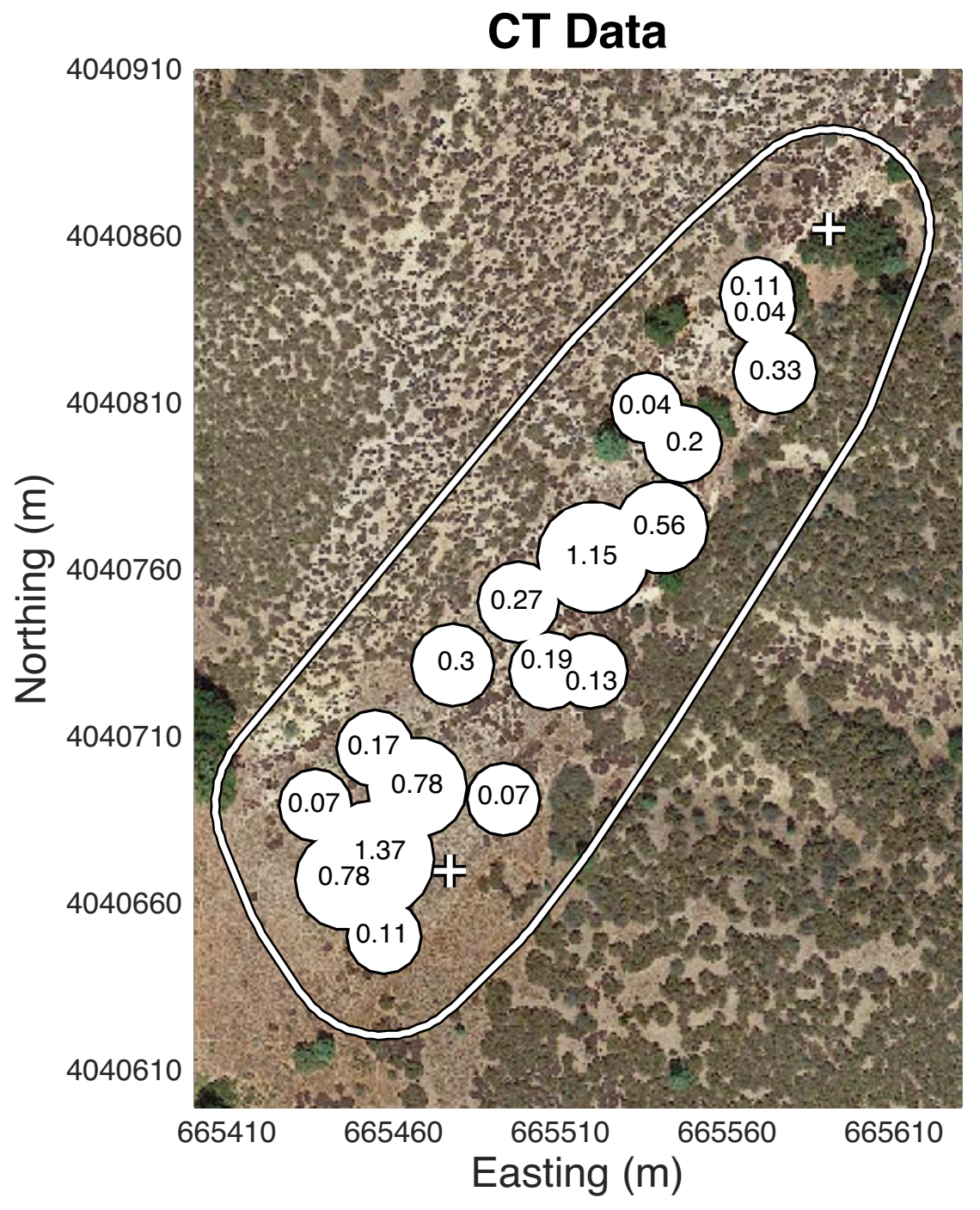

CT-LT Data

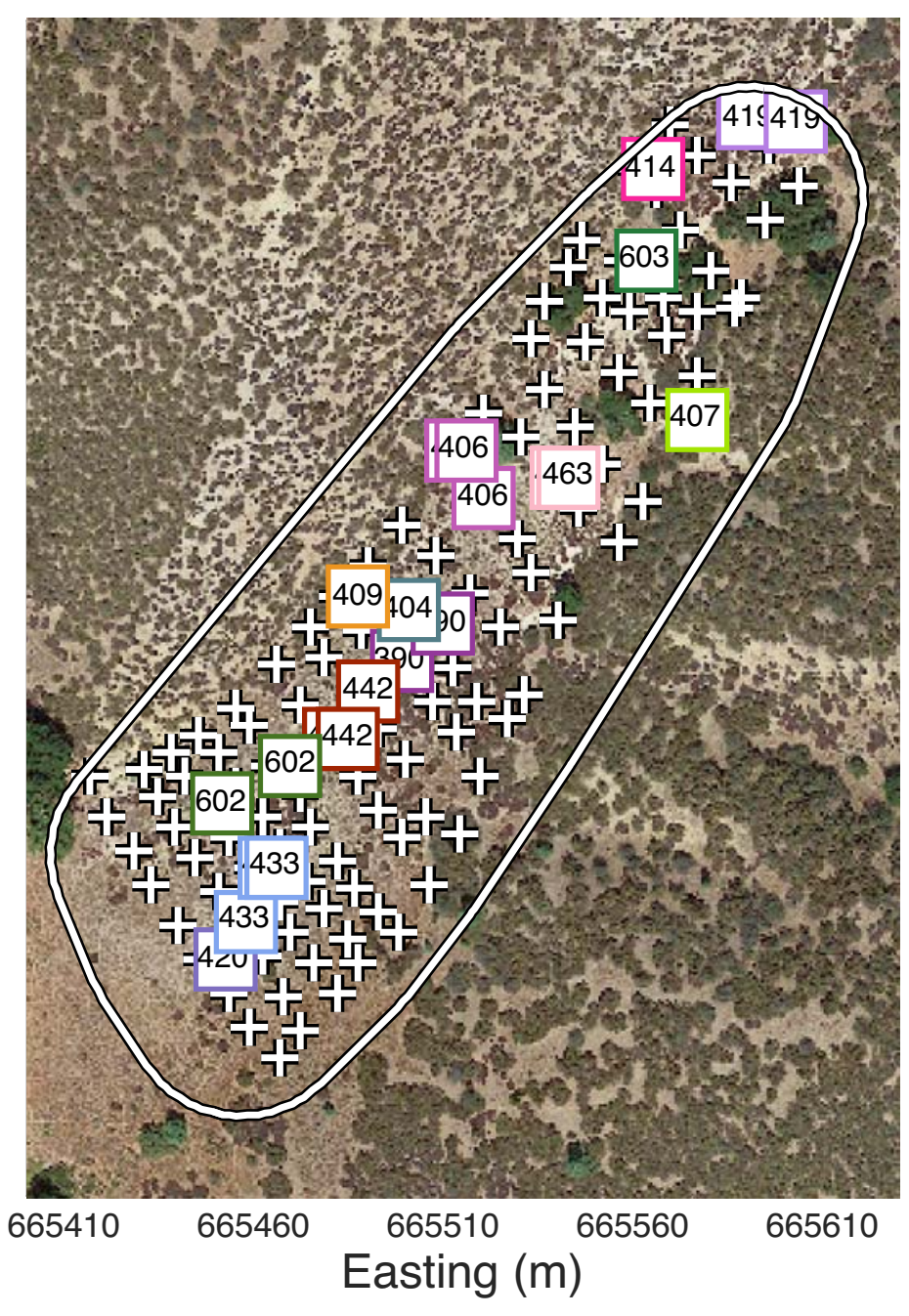




\section{Session S3 Capture Data}
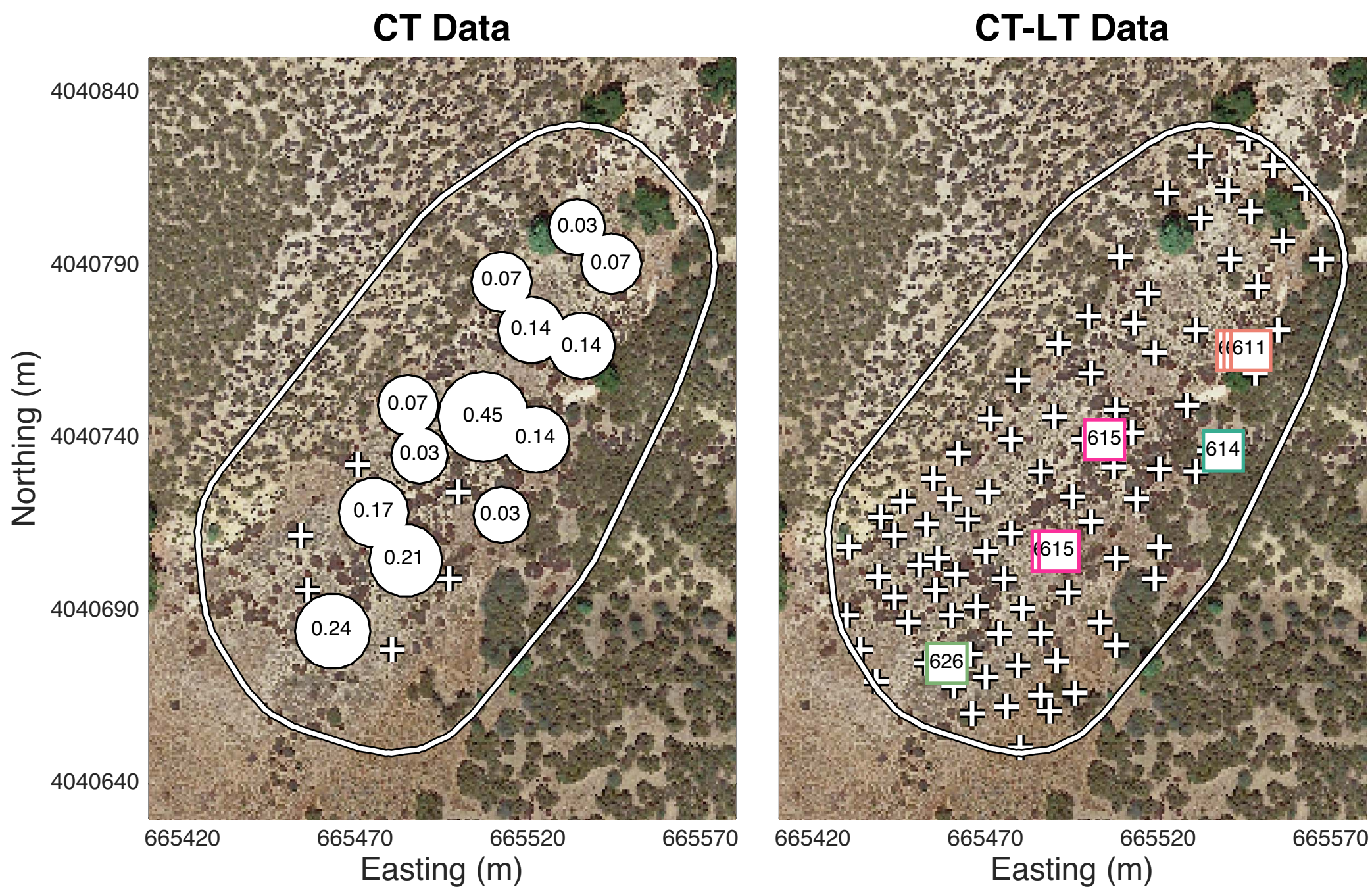


\section{Session S4 Capture Data}
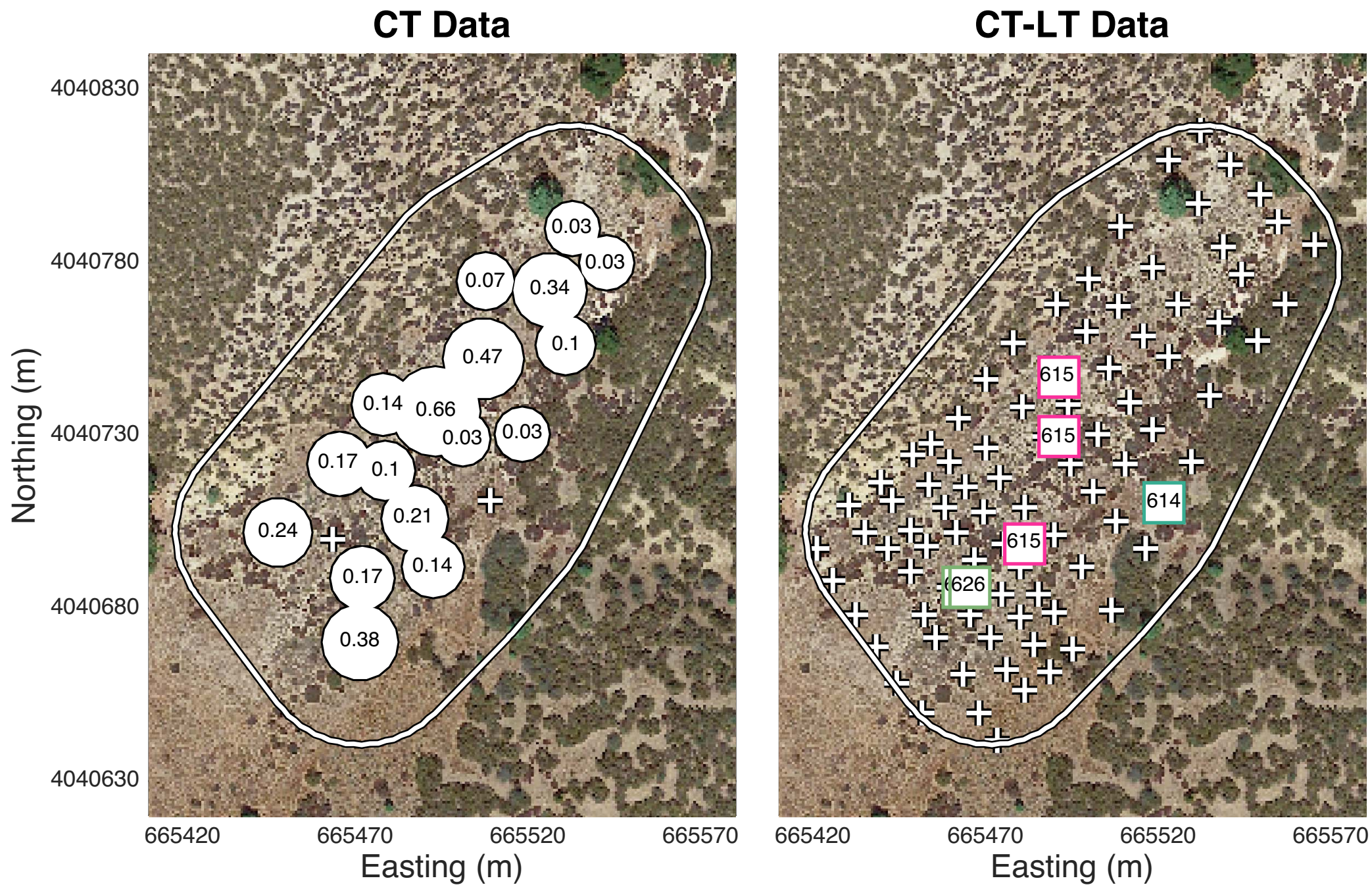


\section{Session S5 Capture Data}
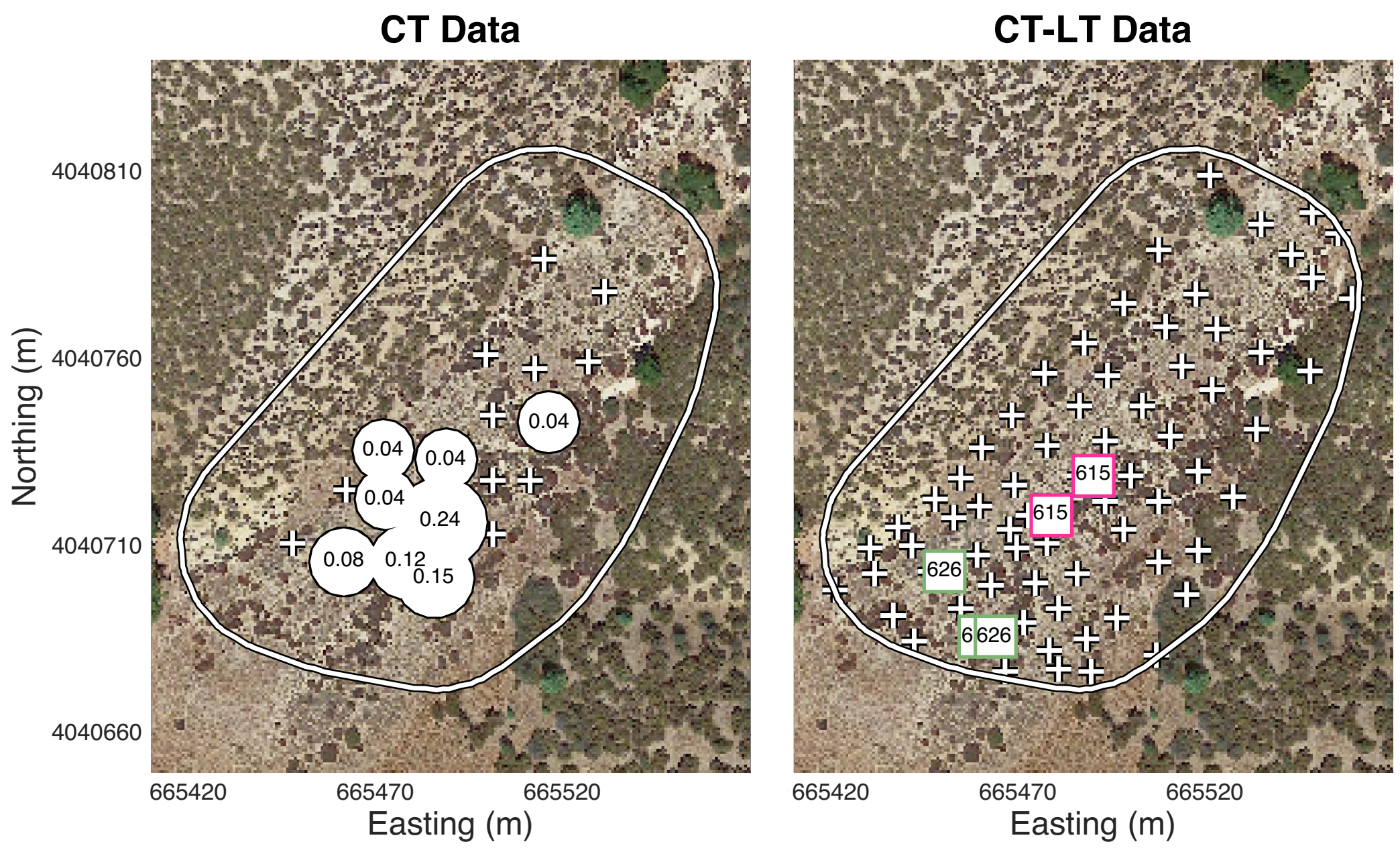


\section{Session S6 Capture Data}
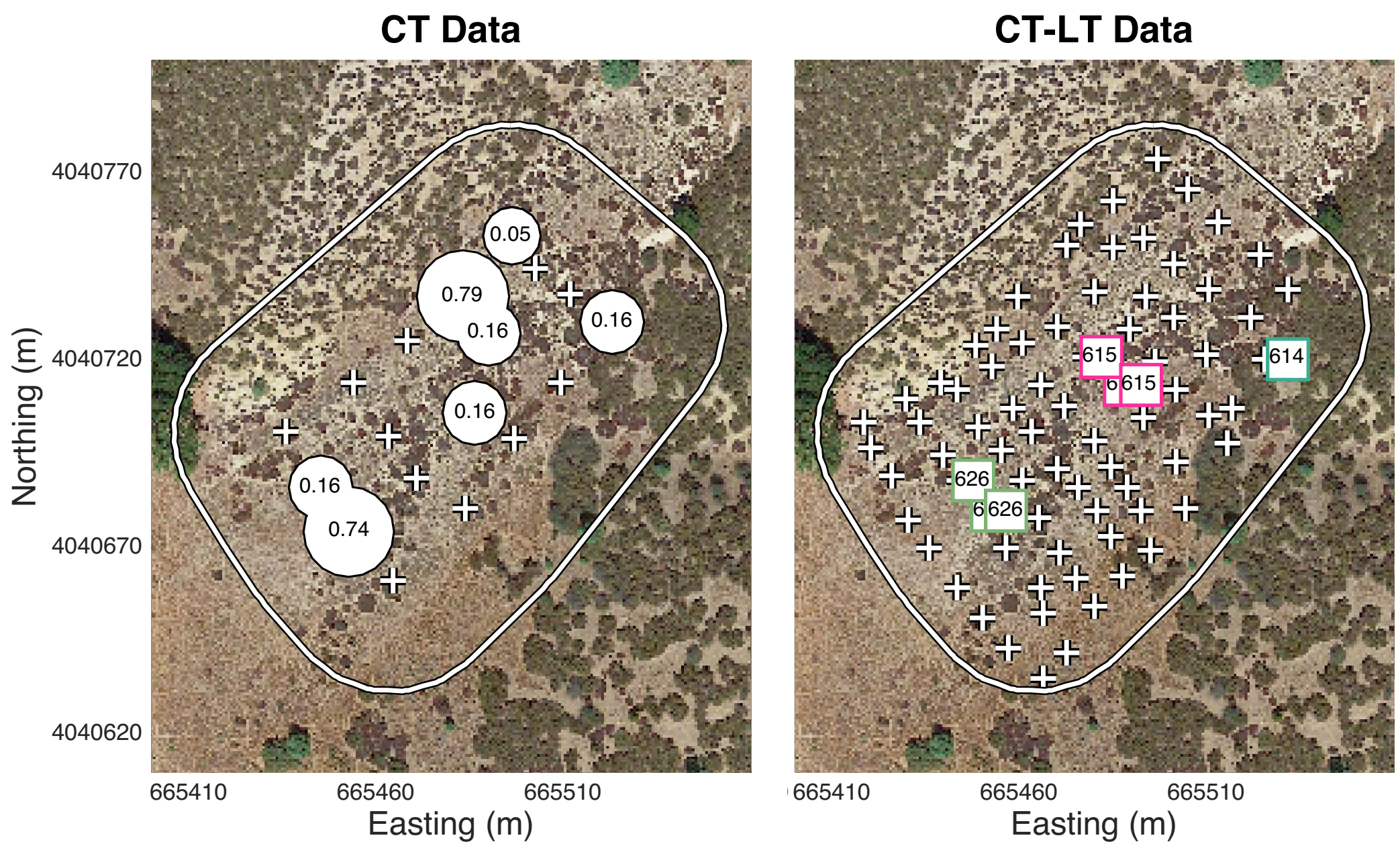


\section{Session S7 Capture Data}
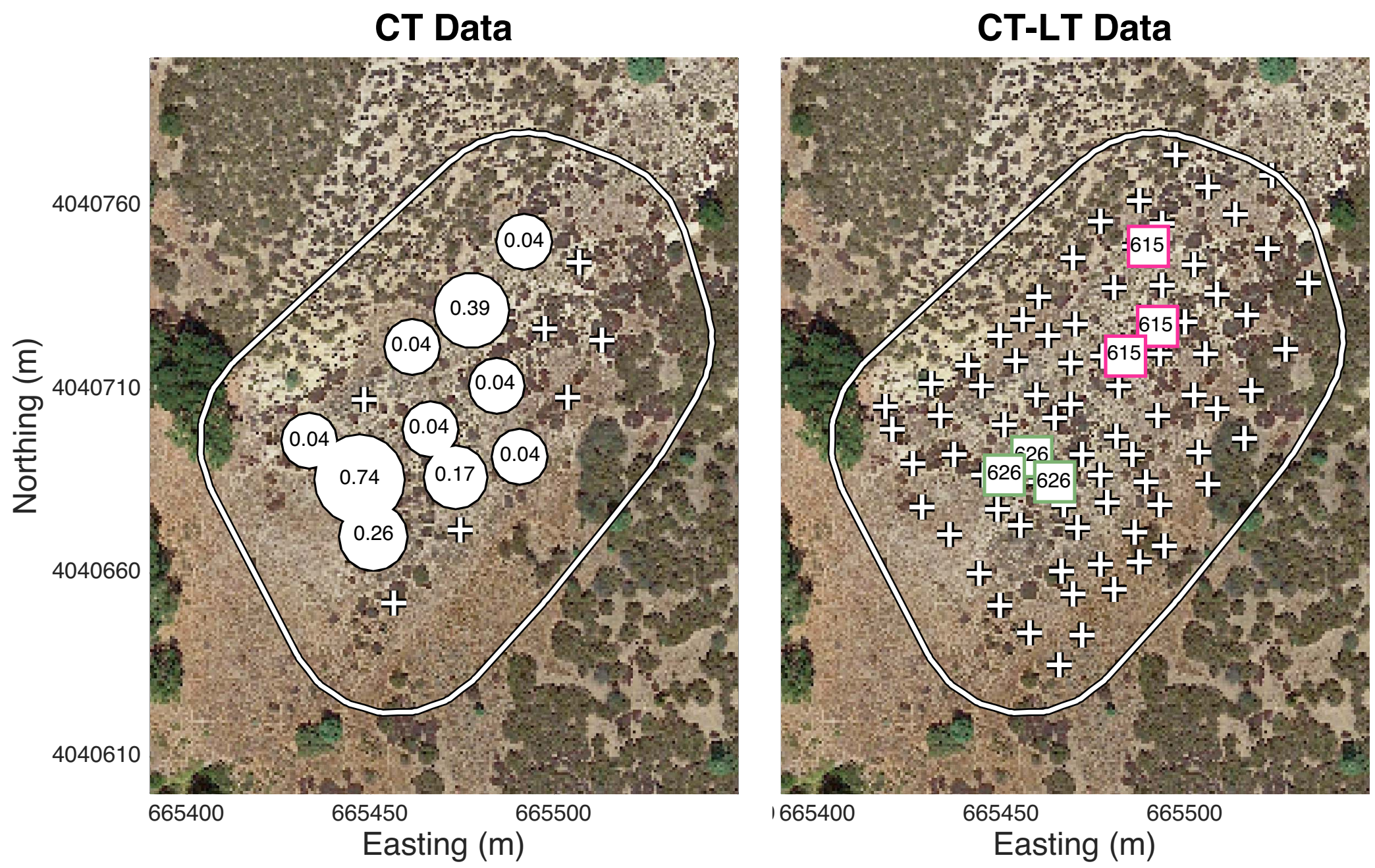


\section{Session S8 Capture Data}
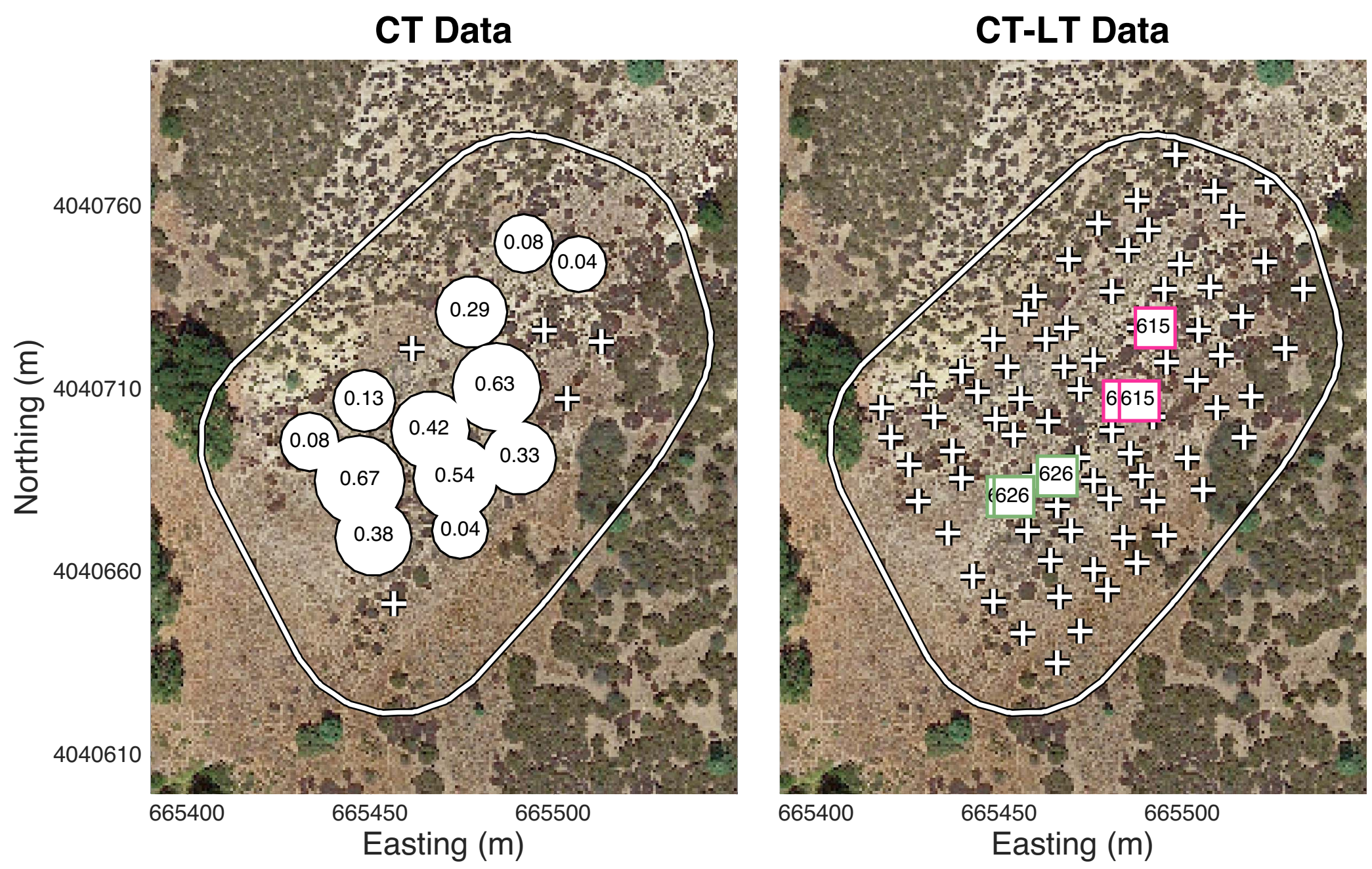


\section{Session S9 Capture Data}
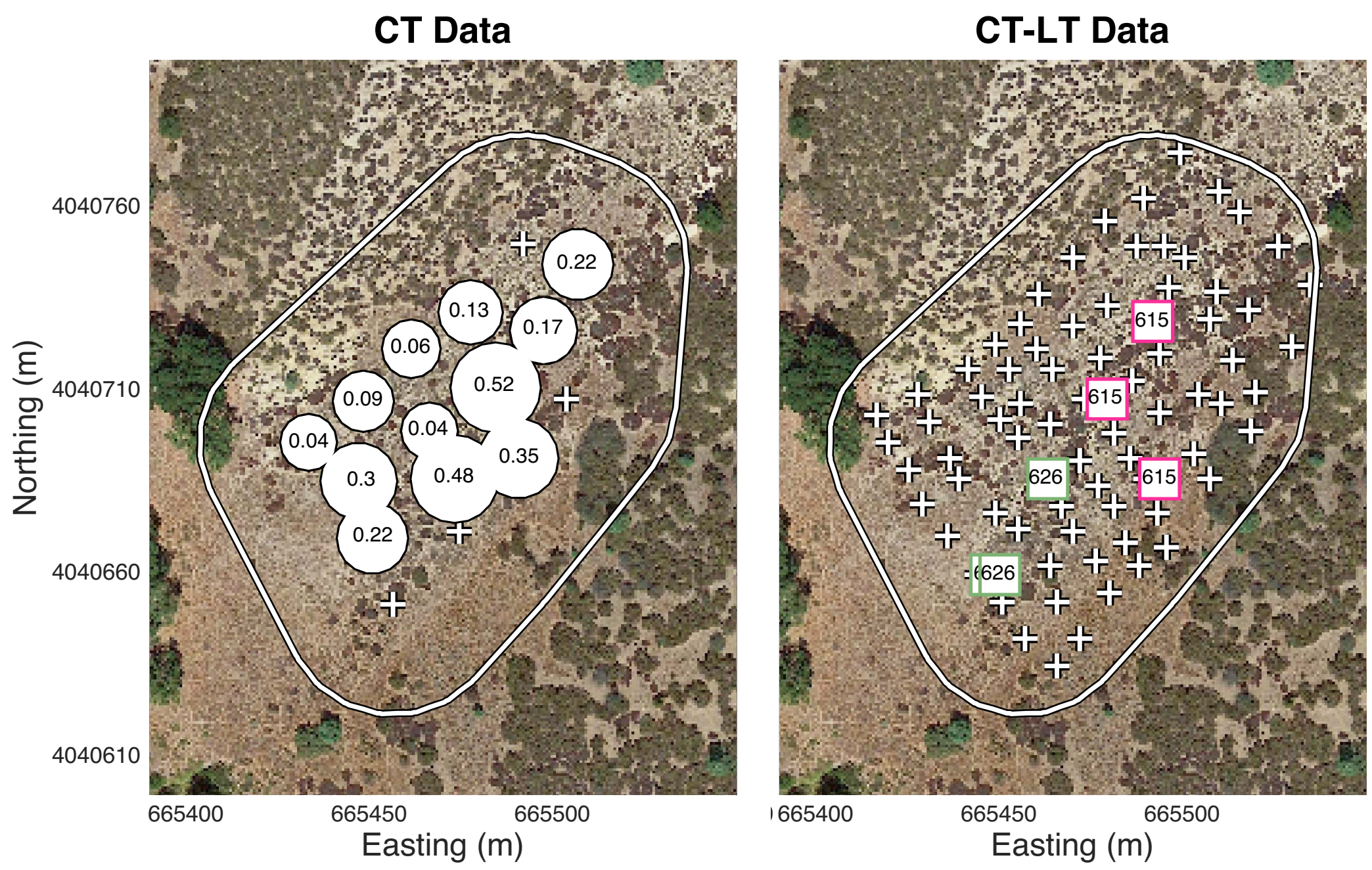


\section{Session S10 Capture Data}
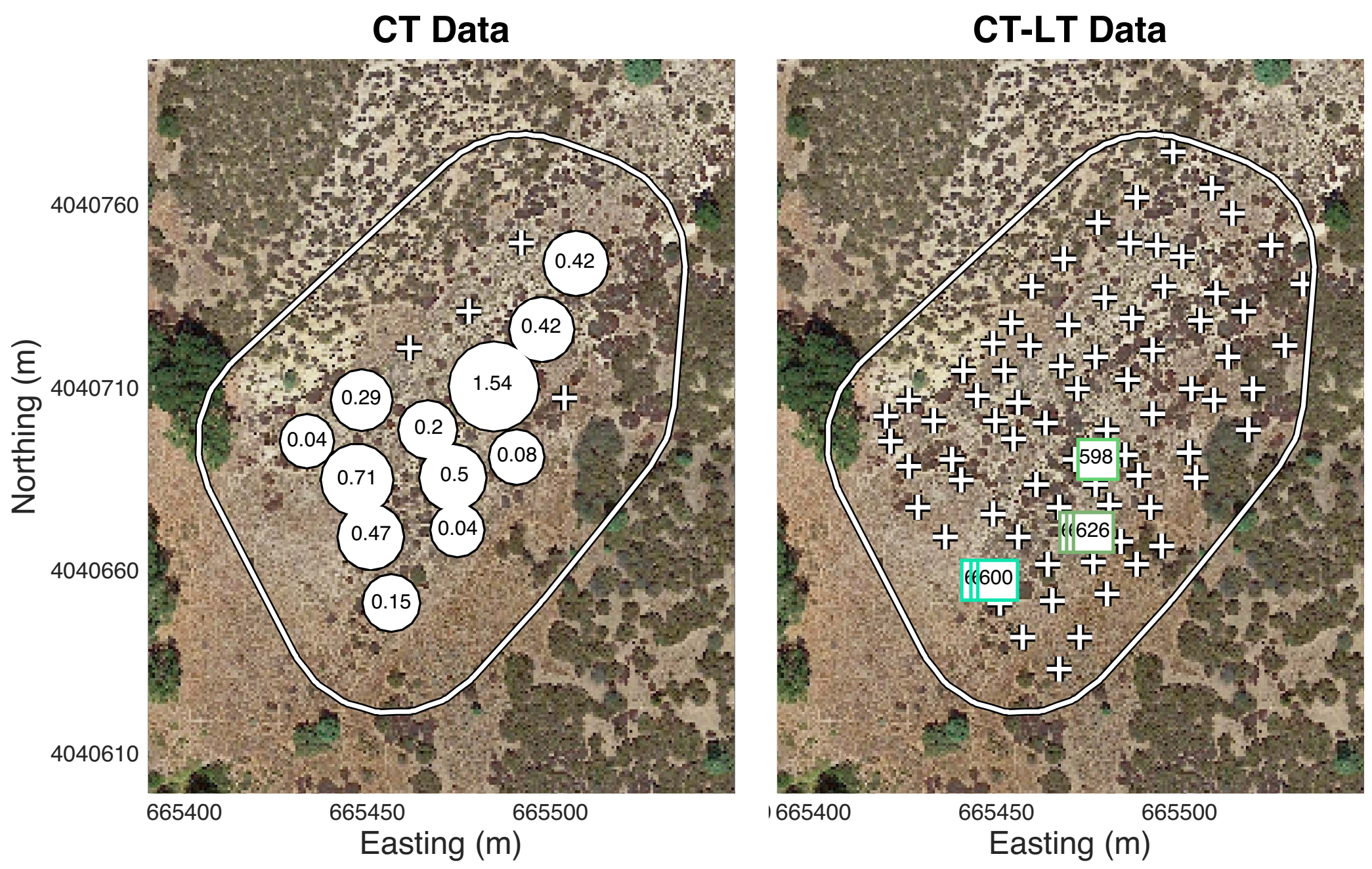


\section{Appendix A3. CTMix and CTMx-LT Capture Histories}

This appendix shows the capture histories, trap locations, and state space polygon for the 10 sessions of combined camera trapping (CTMix) and combined joint trapping (CTMx-LT) datasets. The image below serves as an example. The CTMix capture histories are shown as circles of varying sizes that represent the camera's relative capture rate. The number listed in the center is the average number of captures per day. Some number locations were adjusted for viewing purposes. The CTMx-LT capture histories are displayed the same as the CTMx-LT capture histories but truncated to match the CTMix state space. In the CTMx-LT data individuals captured are represented by their three-digit tag number and a unique colored box. The same box color is also used in the home range center maps in Appendix D. If individuals were captured at the same trap on multiple nights, the tag icons are seen as stacked (for example, see 419 in upper right). Crosses represent all live trap locations $\left(x_{j}\right)$, and the outline shows the state space used in analysis $(S)$.

Underlying imagery (C) 2016 Google Maps, plotted on UTM Zone 10S.

\section{Mix Session S1+S2 Capture Data}
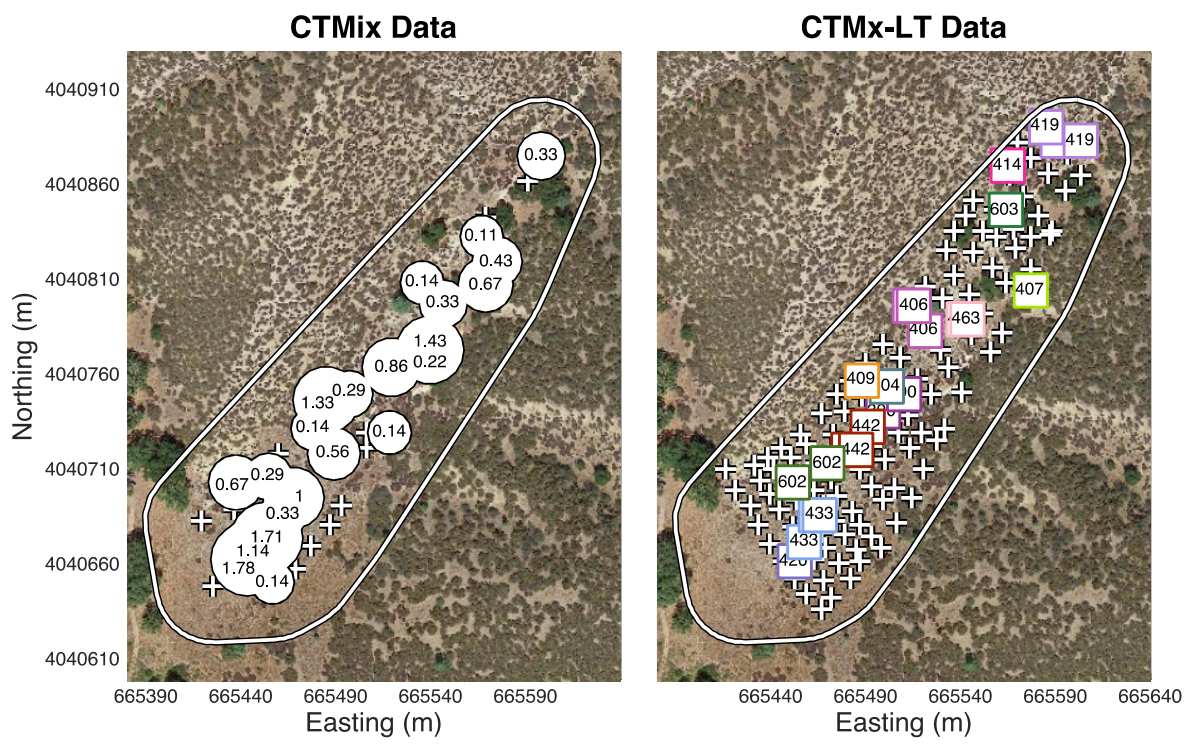


\section{Mix Session S1+S2 Capture Data}
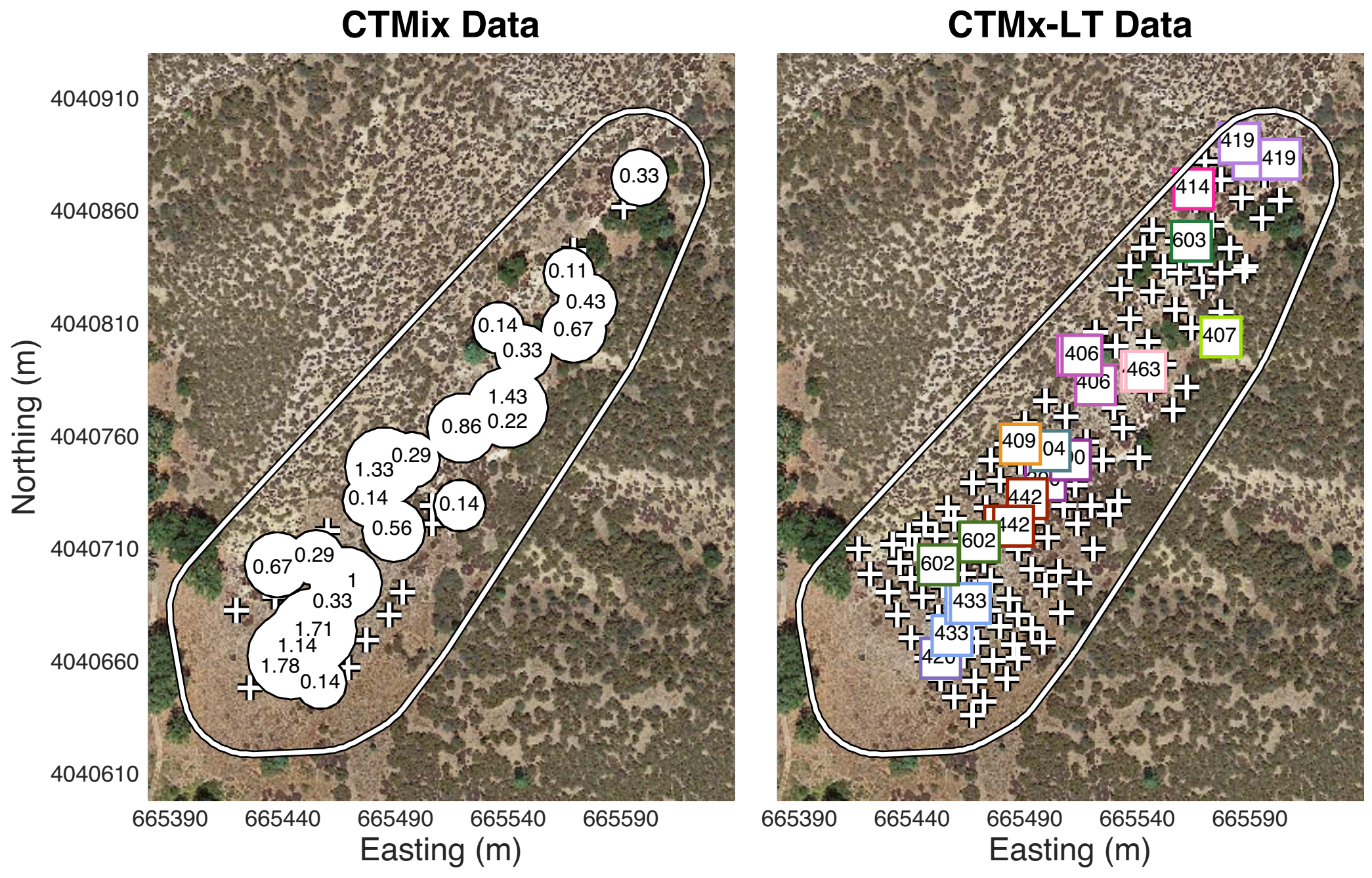
Mix Session S3+S4 Capture Data CTMix Data
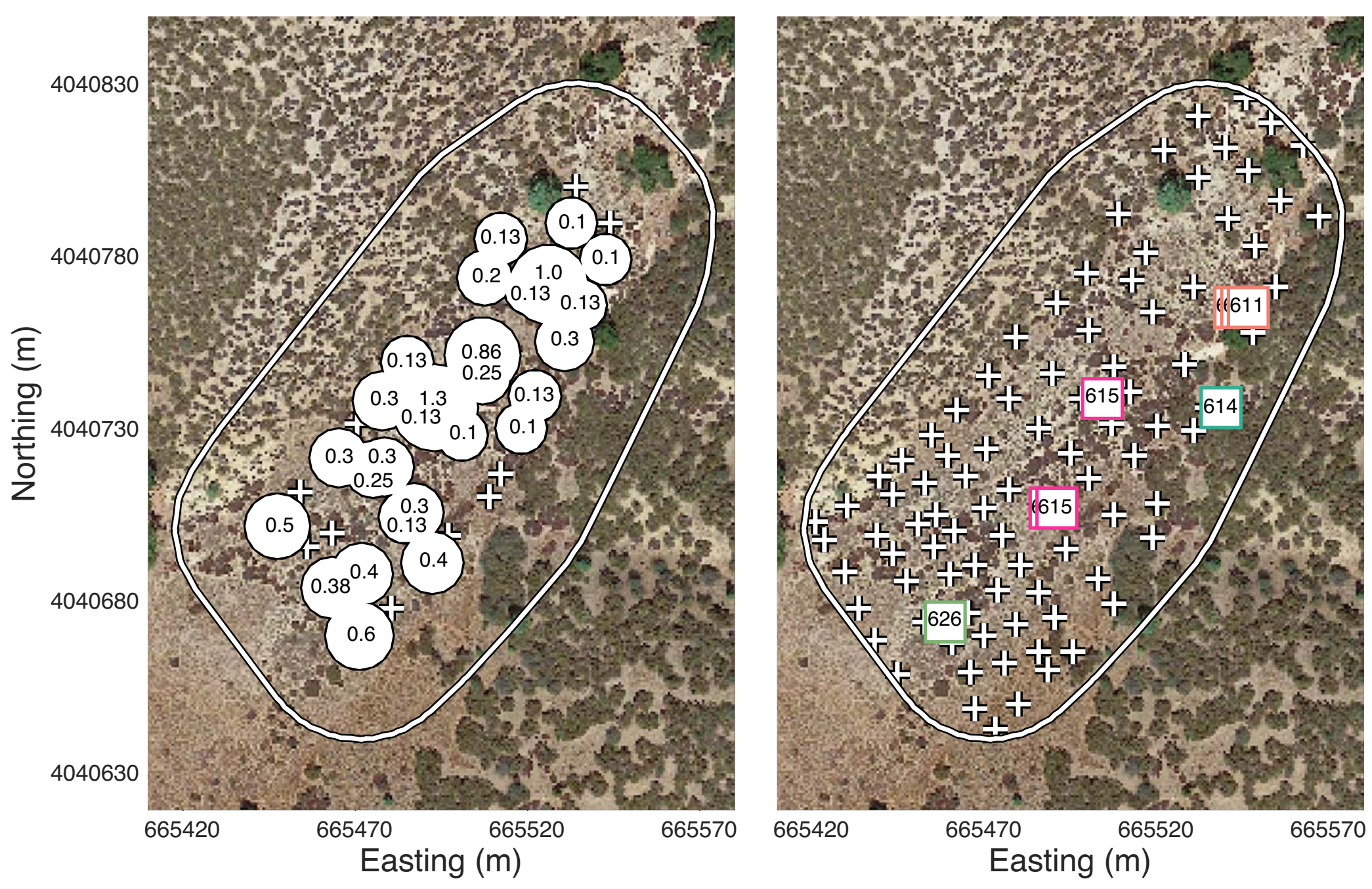


\section{Mix Session S4+S5 Capture Data}

CTMix Data

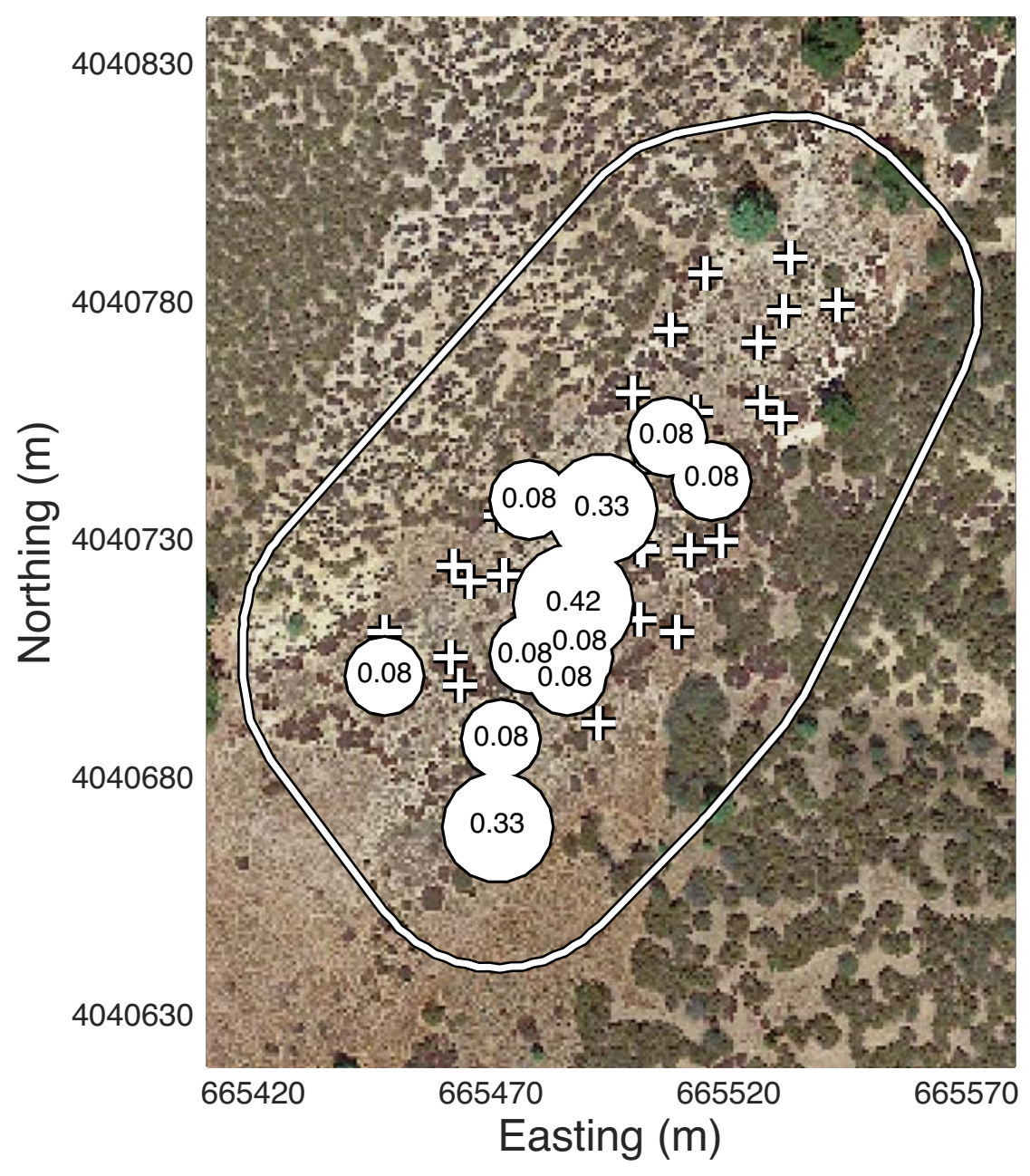

CTMx-LT Data

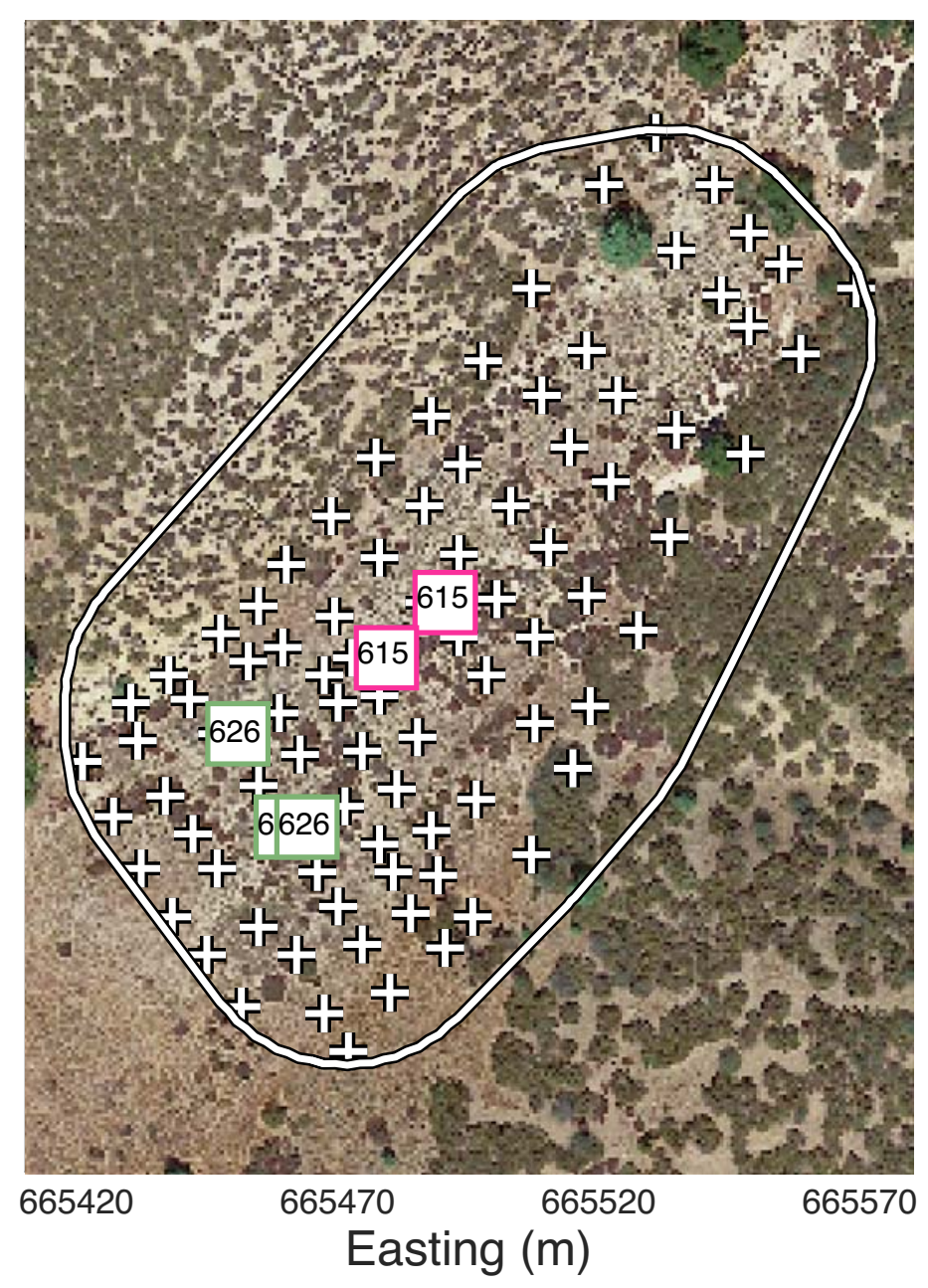




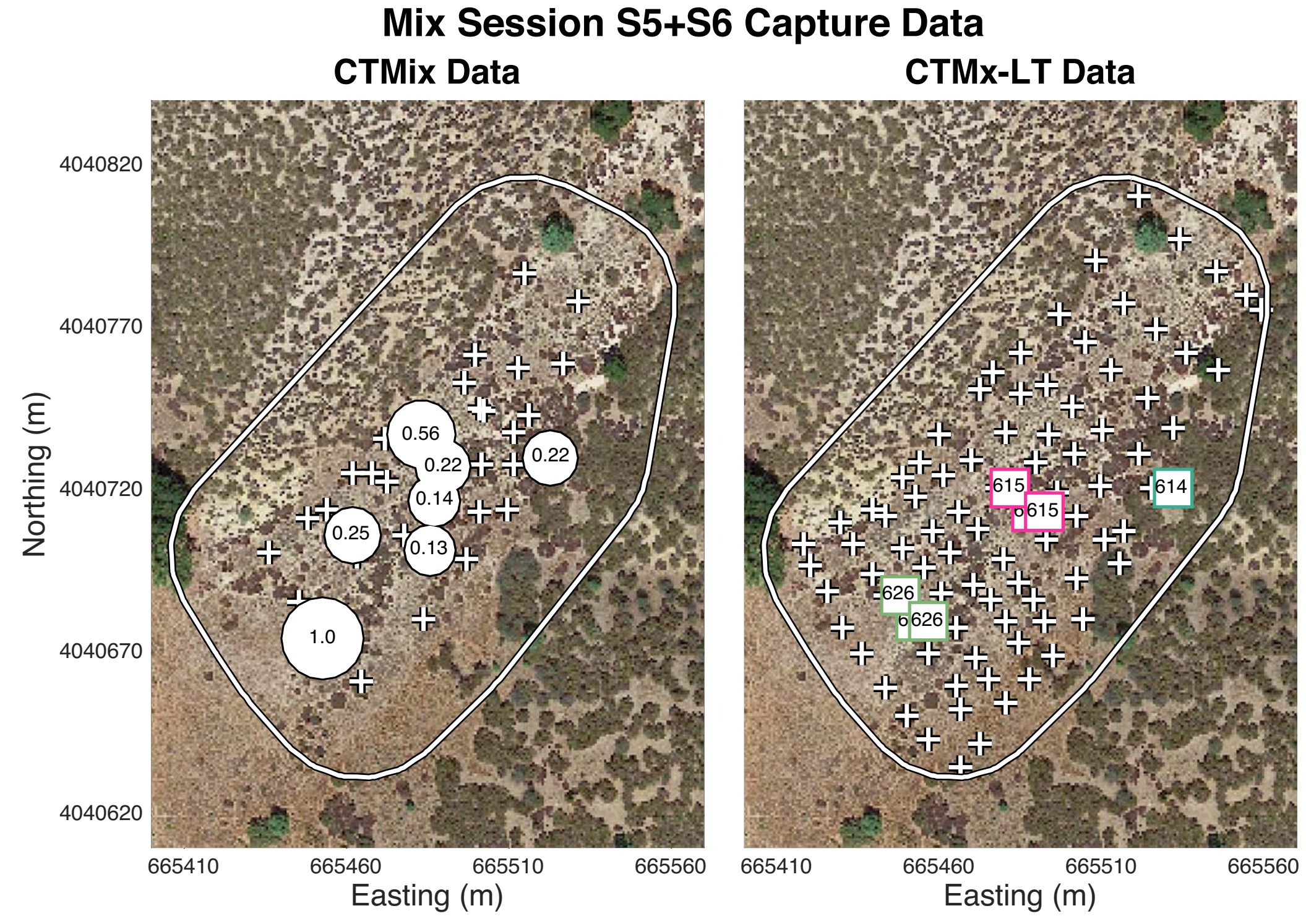




\section{Mix Session S6+S7 Capture Data}
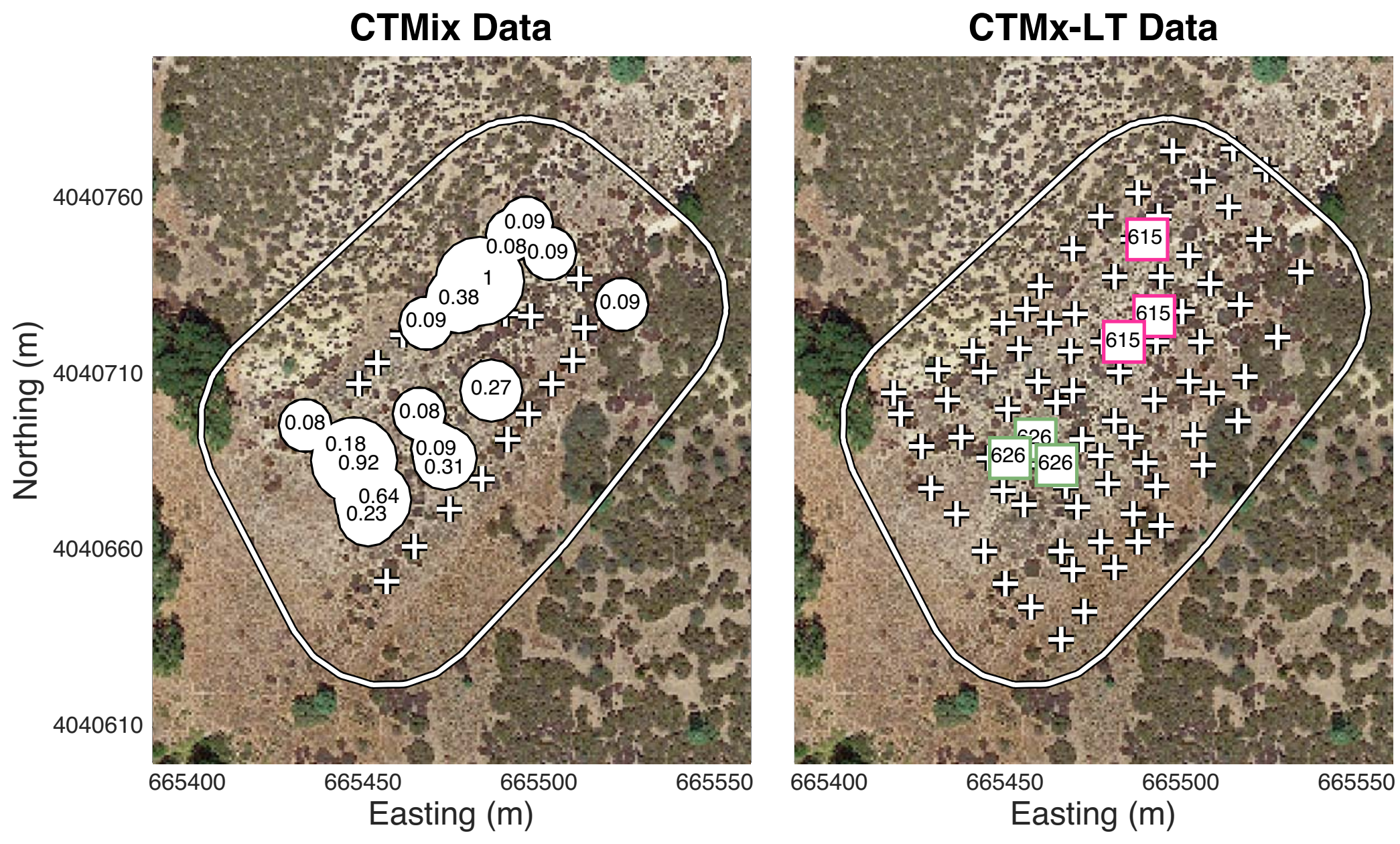


\section{Appendix B. Model Specification}

In this appendix the SC model, the multinomial SCR model, and their associated parameters are described in detail. The appendix is separated into four sections:

1. The Observed Data: Describes how the data are gathered and structured.

2. The Estimated and Chosen Parameters: Describes the basics of SCR models including the parameter values that must be chosen before analysis begins and those that are estimated from the observed data.

3. Calculating the Likelihood Statements: Describes how the observed data are compared against proposed parameter estimates. Descriptions are given for both of the observation models, the Multinomial (live trapping) and the Poisson (camera trapping).

4. Estimation of the Posterior Distributions: Gives a step-by-step description of how the Metropolis-Hasting Markov chain Monte Carlo algorithm works. 


\section{Observed Data}

Both the live trapping and the camera trapping data are evaluated under the same basic SCR structure; however, the data structure varies due to differences in the method of observation. One parameter that both methods share is trap location, which is denoted as $\boldsymbol{x}_{\boldsymbol{j}}$. Each of the $J$ element's in $\boldsymbol{x}_{\boldsymbol{j}}$ takes the form of an $\mathrm{X}$ and $\mathrm{Y}$ coordinate.

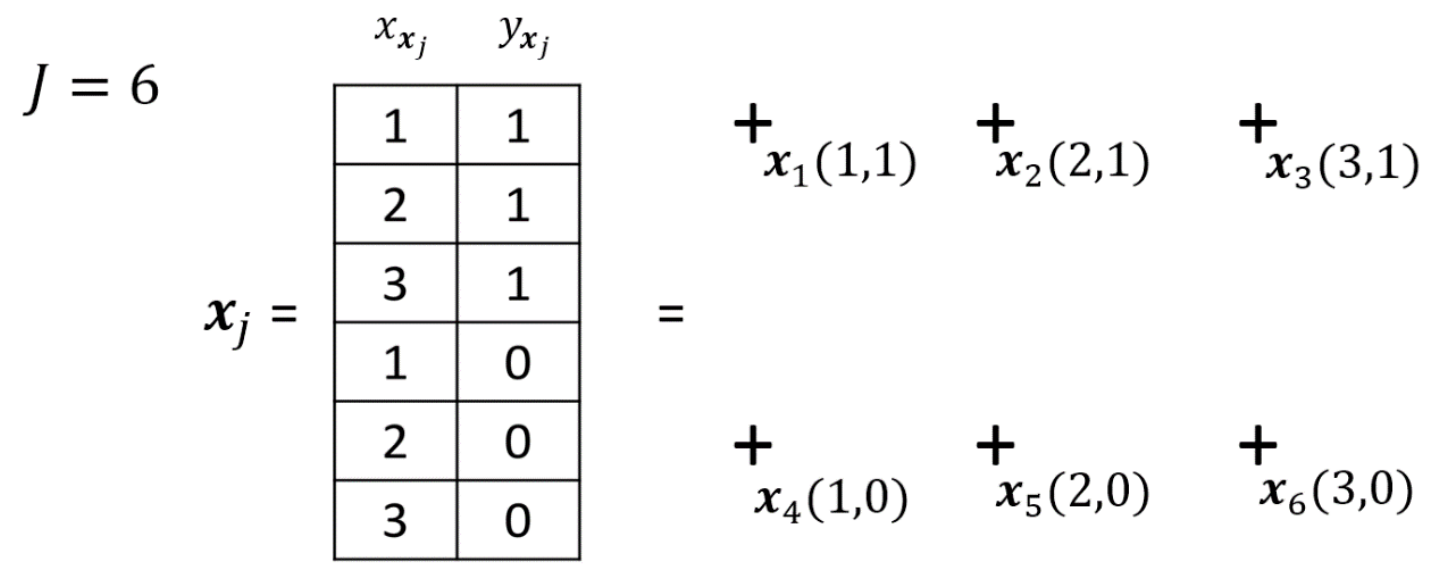

For the live trapping data, each of the $n$ individuals captured are recorded in the parameter, $y_{i j}$, which is an $n$ by $J+1$ matrix of the number of times and individual $i$ is capture at trap $j$. This data is recorded over the course of $K=3$ trapping nights. If an individual was not captured at any trap on a given night, a value is added to the $J+1$ position of the matrix.

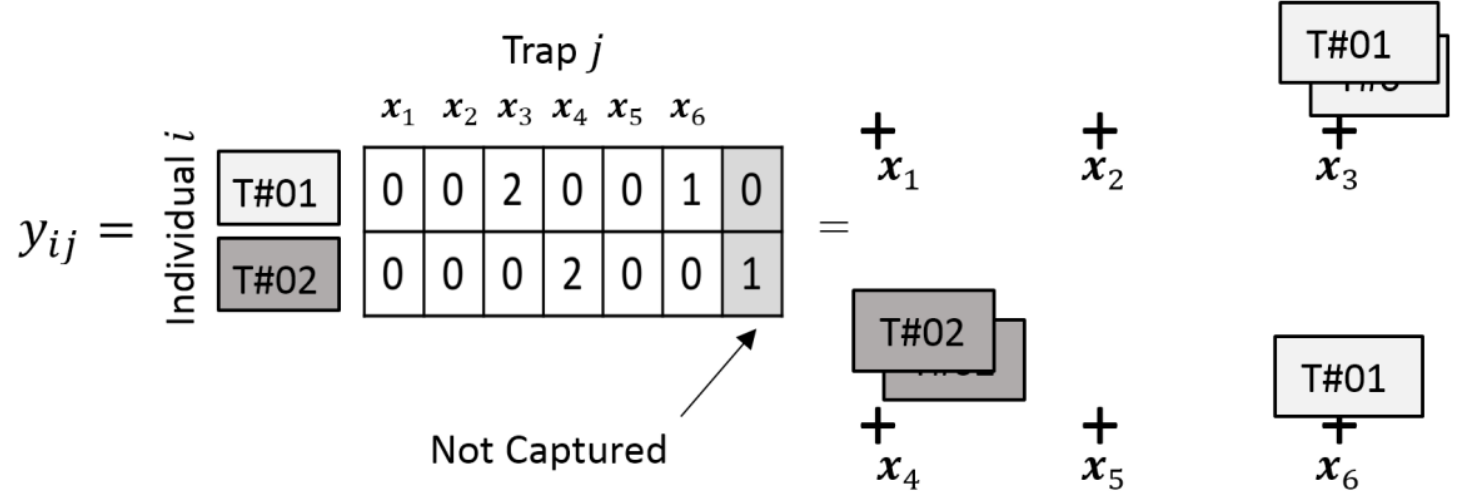


For the camera trapping data, individual identities are unknown, but each of the $T$ days are retained in the capture data. Thus, the camera data parameter, $y_{t j}$, is a $T$ by $J$ matrix recording how many capture events were recorded at $\operatorname{trap} j$, on day $t$. An additional parameter, $c_{j t}$, is also recorded to account for days when particular cameras were nonoperational. On days when a camera was operational $c_{j t}=1$, and on days when a camera was non-operational $c_{j t}=0$.

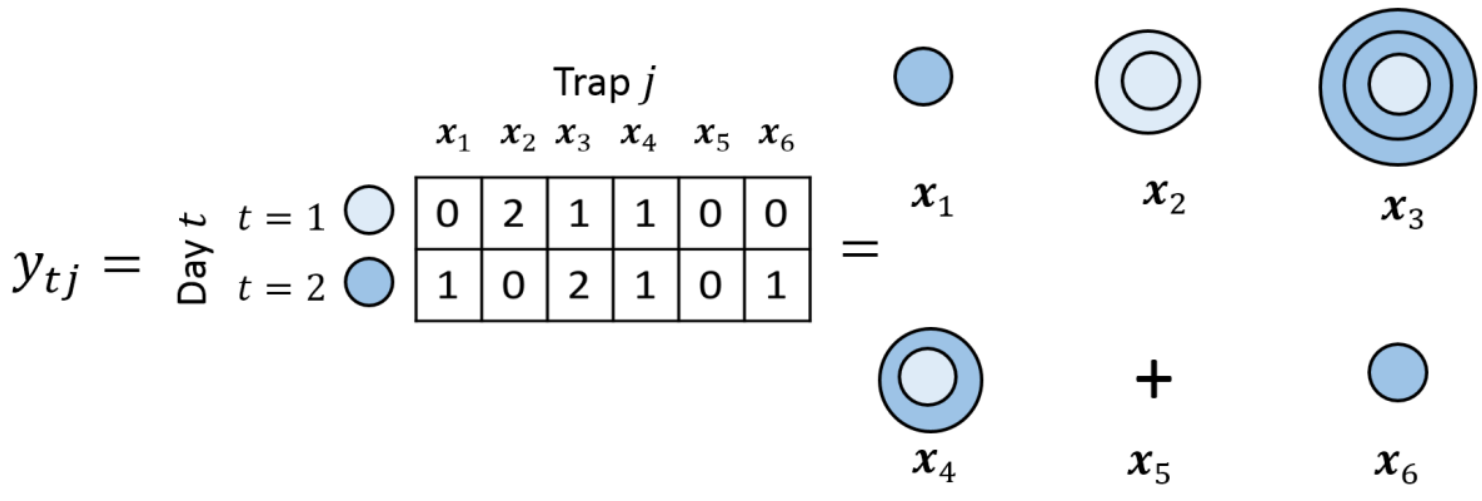

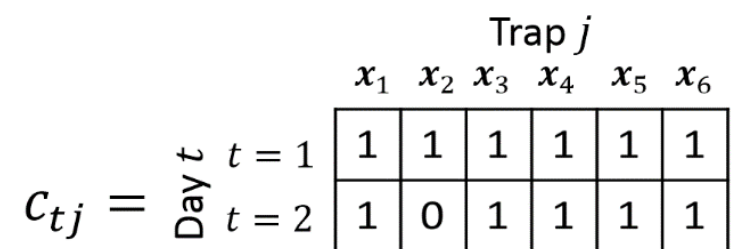

\section{Estimated \& Chosen Parameters}

SCR models estimate animal density by explicitly stating an animal movement model as well as a trapping observation model. Animal movement is described in SCR models as a decreasing function of distance from a hypothetical home range center. The parameter $\boldsymbol{s}_{i}$ represents the coordinates of individual $i$ 's home range center. Home range centers are realizations of a point process with a uniform distribution across a state space, $S$, such 
that $\boldsymbol{s}_{i} \sim$ Uniform $(S)$. The state space is analogous to the effective trapping area used in traditional CR models. In general, so long as $S$ is sufficiently large, density results are thought to be unbiased. The use of a uniform prior on $\boldsymbol{s}_{i}$ keeps animal home range centers independent of one another in the model, allowing them to take on the form of both clumped and even distributions in space.

The probability of encountering a specific individual at a specific trap is described by a detection function evaluated at the distance between the home range center, $\boldsymbol{s}_{i}$, and the trap location, $\boldsymbol{x}_{\boldsymbol{j}}$. The distance between $\boldsymbol{s}_{i}$ and $\boldsymbol{x}_{j}$ is denoted as $\left\|x_{j}-s_{i}\right\|$, which represents the Euclidean distance between the two points (i.e. $\sqrt{\left(x_{x_{j}}-x_{s_{i}}\right)^{2}+\left(y_{x_{j}}-y_{s_{i}}\right)^{2}}$ ).

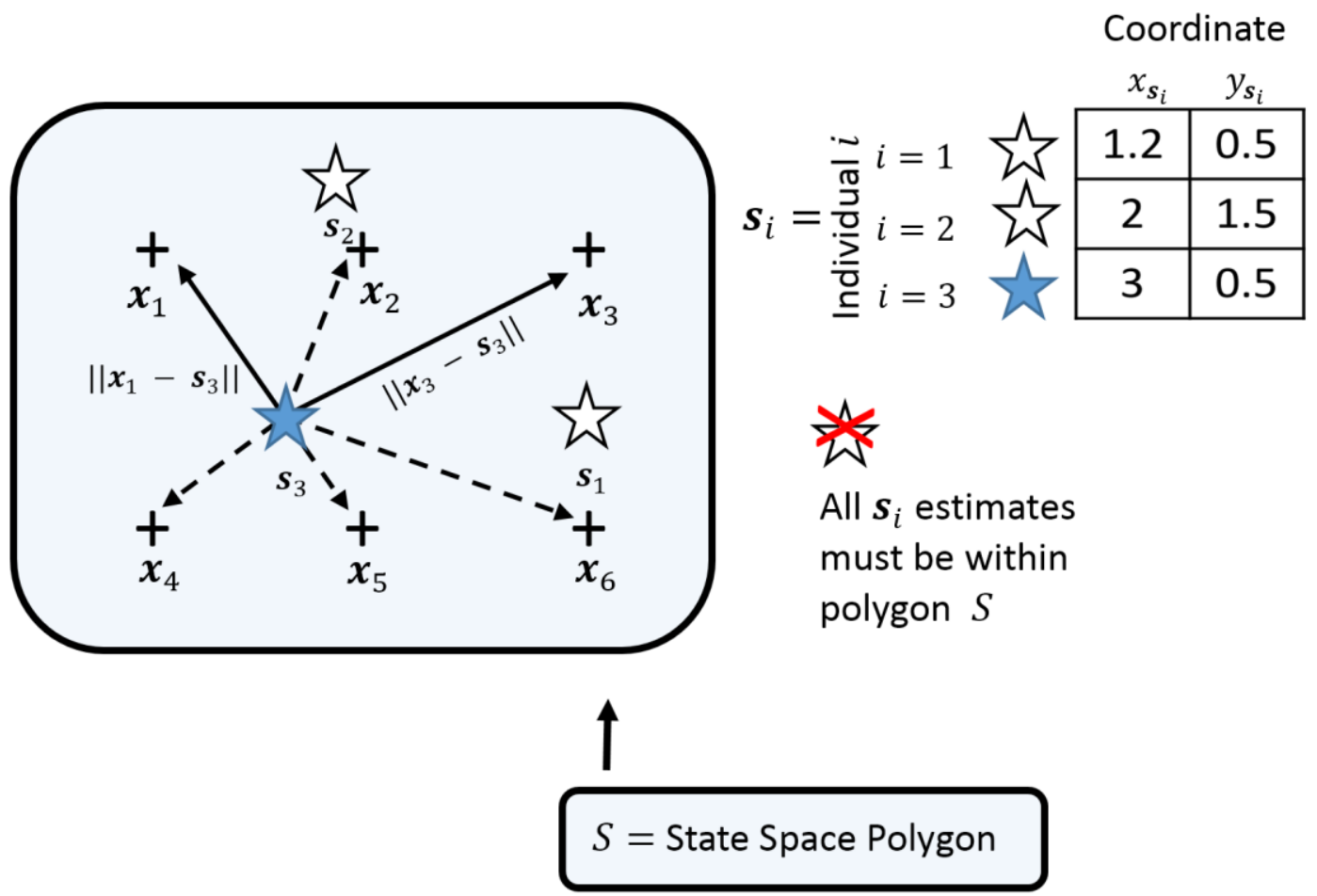

Any monotonically decreasing function may serve as a detection function and several variations have been suggested in the SCR literature including, but not limited to, the half 
normal, exponential, and step functions. All of these functions describe the relative probability of a being captured at a specified distance from a home range center given a home range size parameter, $\sigma$. For this study, a new detection function was developed that could approximate the functions listed above while placing the emphasis on biologically relevant features of the function. Previously, $\sigma$ had different interpretations for different detection functions. Home range size was then estimated from $\sigma$, dependent on the function used. For instance, with the half normal detection function, home range radius was approximated as $2.45 \sigma$. To unify the detection functions and create a more stable interpretation of $\sigma$, a new detection function was developed and is given as: $d\left(x_{j}, s_{i}\right)=\exp \left(z \frac{\left\|x_{j}-s_{i}\right\|^{a}}{\sigma^{a}}\right)$. The graph below shows its key features.

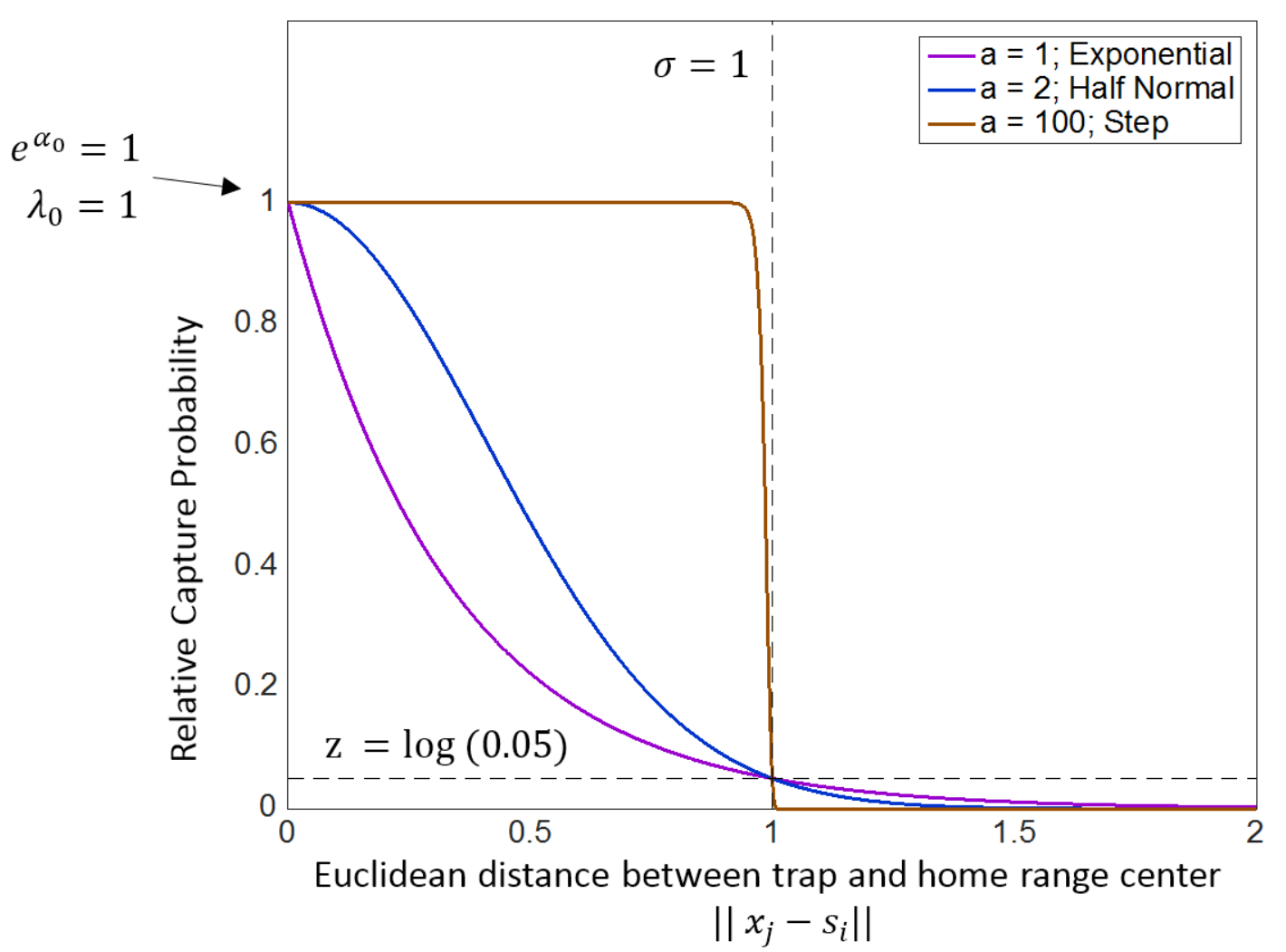


With this detection function, the home range radius is explicitly defined as the distance from a home range center when the detection function reaches a sufficiently low probability of capture. This low point is controlled by $z=\log \left(p_{c}\right)$ where $p_{c}$ is defined by the researcher and represents the probability of capture at a distance of $\sigma$ from the home range center $\boldsymbol{s}_{i}$ when the encounter rate parameter is set to 1 . Parameter $a$ controls the shape of the function. When $a$ is set to 1 , the detection function resembles the exponential detection function; when $a$ is set to 2 , it resembles the half normal detection function; and if $a$ is set high (e.g., 100), it resembles the step function.

In the figure above, two additional parameters are given, $\lambda_{0}$ and $e^{\alpha_{0}}$. These are encounter rate parameters and their definitions change depending on the observation model (e.g. live trapping or camera trapping). These parameters are described below. Abundance, $N$, is estimated through Parameter Expanded Data Augmentation (DA), which allows for a model formulation conditional on $N$. DA estimates $N$ using a set of $M$ Bernoulli trials with probability of success $\psi$. For unbiased results, $M$ must be larger than any plausible value of $N$. A new parameter, $w_{i}$, is made up of $M$ binary indicator variables where $w_{i}=1$ if individual $i$ is truly a member of the population and $w_{i}=0$ if not. Abundance is then calculated as $N=\sum_{i=1}^{M} w_{i}$. DA emphasizes estimating $\psi$, which is approximately $\frac{E[N]}{M}$. In the MCMC algorithm, $\psi$ is updated from a beta distribution $\beta(N+1, M-N+1)$, effectively placing a discrete uniform prior on $\mathrm{N}$, such that $N \sim \operatorname{DUnif}(0, M)$. DA requires $y_{i j}$ to be expanded with $M-n$ all-zero capture histories. 


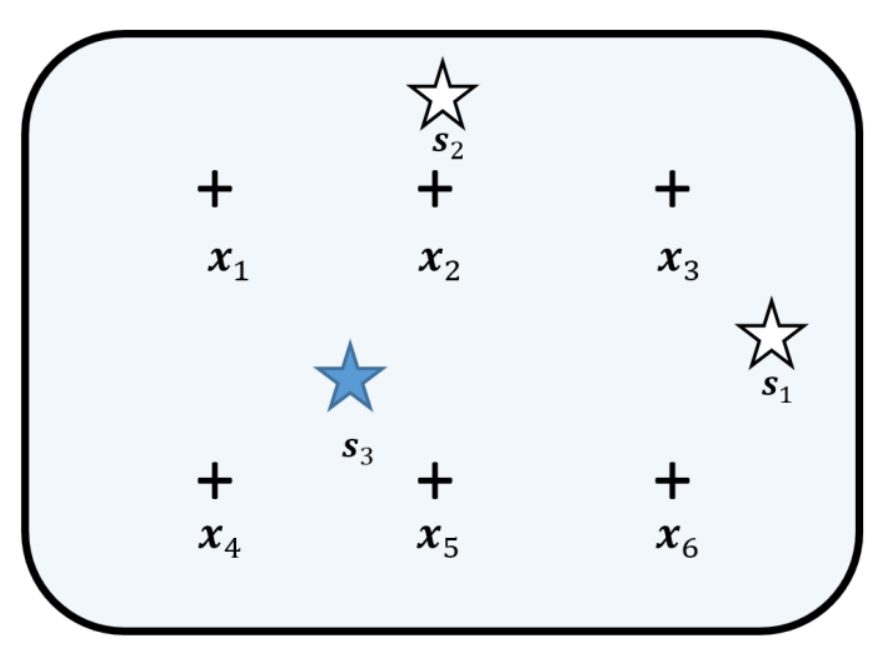

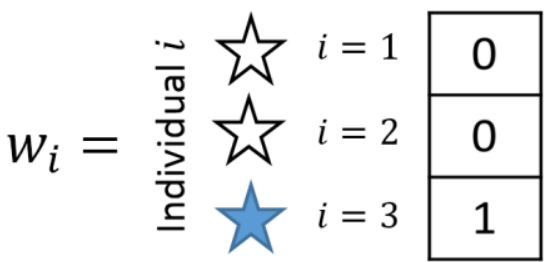

$$
\begin{aligned}
& M=3 \quad N=\sum_{i=1}^{M} w_{i}=1 \\
& \psi=\frac{E[N]}{M}=1 / 3
\end{aligned}
$$

Instances where $w_{i}$ is estimated as one in the expanded indices of $y_{i j}$ represent sampling 0 's, where an individual is present but unobserved. Since no individual identity is recorded in the camera data, it does not need to be expanded in the same way; though DA is still used in the estimation of $N$. Although it may seem strange to have extra individuals in the estimation process which are often turned off and unused in the estimation process, their presence is critical as it allows the MCMC algorithm to explore many different possibilities of both $\boldsymbol{s}_{i}$ and $N$.

\section{Calculating the Likelihood Statements}

Calculating the full conditional likelihood, $\mathcal{L}_{L T}$ and $\mathcal{L}_{C T}$, is a critical step in the Metropolis-Hasting MCMC algorithm. The likelihood statements for the live trapping and the camera trapping models are very different, but both use the same underlying SCR movement model. The difference is in the type of data collected by each trapping method. In live trapping, each animal present can be captured in at most one trap on each of the three occasions. This type of data can be described by a multinomial observation model. 
In camera trapping, the traps do not hold the animal, and so animals are free to encounter multiple traps many times on a given occasion. This type of data can be described by a Poisson observation model. Since both data types are ultimately described by known probability distributions, the Poisson and the Multinomial distributions, the probability mass functions of the two distributions can be used to evaluate the full conditional likelihoods. These likelihood statements are used to assess how likely a given set of parameters are (e.g., $\left.\boldsymbol{s}_{i}, \sigma, w_{i}, N, \psi, \lambda_{0} / \alpha_{0}\right)$ compared to the observed data $y_{i j}$ and $y_{t j}$. Both distributions evaluate the observed data given a proposed set of expected values that are calculated from the estimated parameters. For the multinomial observation model, the expected values are given as the matrix $\pi_{i j}$, and for the Poisson observation model, the expected values are the matrix $\lambda_{t j}$.

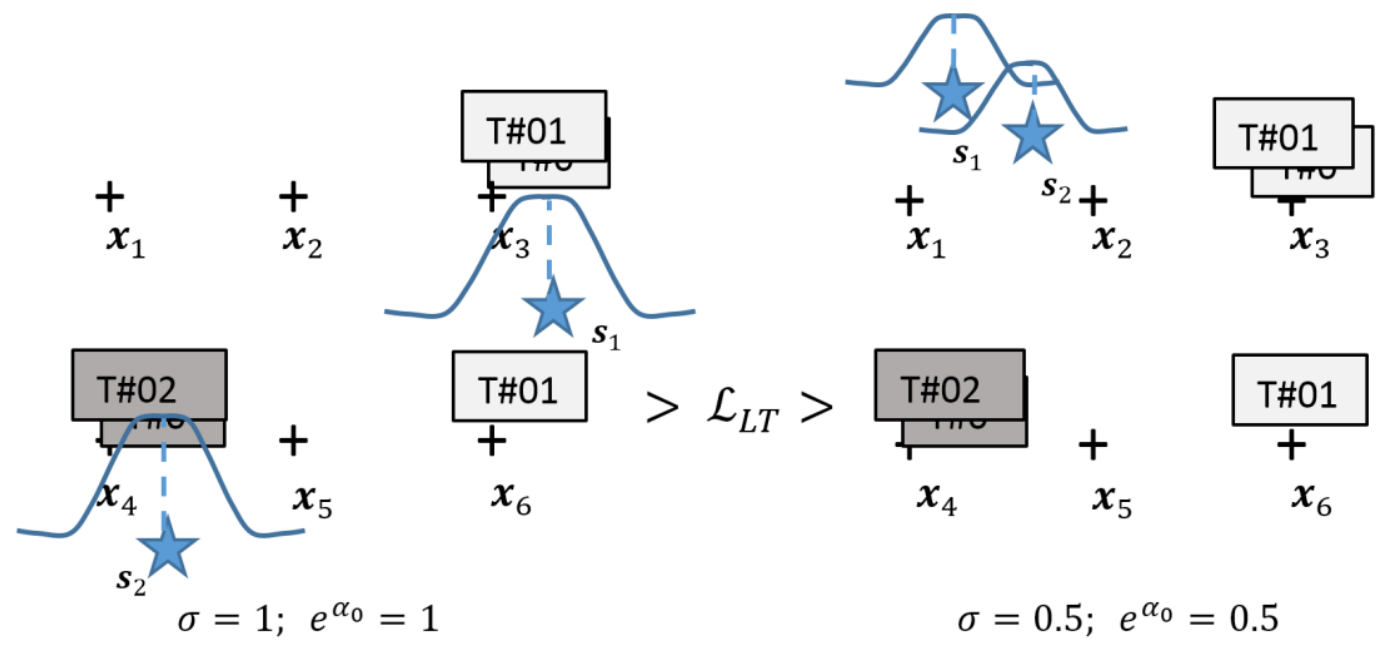

Parameter estimates that better explain the observed data produce a higher likelihood value. Using the live trapping data presented above as an example, it is clear that the location of the home range centers, home range radius, and activity levels 
presented on the left, match with the observed data better. Because the left matches better, the likelihood, $\mathcal{L}_{L T}$, would be larger than in the case on the right.

Under the multinomial observation model used for the live trapping data, the expected values, $\pi_{i j}$, describe the probability that an individual $i$, is captured at trap $j$, or not at all. The probabilities of being caught in a particular trap are calculated as:

$$
\pi_{i j}=\frac{\exp \left(\alpha_{0}\right) d\left(x_{j}, s_{i}\right)}{1+\sum_{j=1}^{J} \exp \left(\alpha_{0}\right) d\left(x_{j}, s_{i}\right)}
$$

The probability of not being captured in a trap is calculated in the $J+1$ position as:

$$
\pi_{i J+1}=\frac{1}{1+\sum_{j=1}^{J} \exp \left(\alpha_{0}\right) d\left(x_{j}, s_{i}\right)}
$$

Here, $\alpha_{0}$ represents the capture rate parameter for the multinomial observation model. As $\alpha_{0}$ increases, the probability of not capturing an individual at a trap decreases. In this sense, it can be thought of as average capture rate parameter. For every individual, $i$, there must be a $100 \%$ chance of falling into one of the $J+1$ categories, such that $\sum_{j=1}^{J+1} \pi_{i j}=1$ for all values of $i$

The likelihood of each of the $M$ individual's capture histories, $y_{i j}$, given a set of expected values, $\pi_{i j}$, is denoted as $\mathcal{L}_{i}$ and is calculated by using the multinomial probability mass function. The probability is only calculated for individuals where $w_{i}=$ 1 ; if $w_{i}=0$, the index is ignored.

$$
\mathcal{L}_{i}=\operatorname{Multinomial}\left(y_{i j} \mid K, \pi_{i j}, w_{i}\right)=\frac{K !}{y_{i 1} ! \cdot y_{i J+1} !} \pi_{i 1}^{y_{i 1}} \cdots \pi_{i J+1}^{y_{i j+1}} \text {, when } w_{i}=1
$$

The full conditional likelihood, $\mathcal{L}_{L T}$, is then calculated as the product of all the values of $\mathcal{L}_{i}$. However, due to rounding problems which occur using computers to 
evaluate very small values, like the probabilities which occur here, a log conversion of the likelihood is implemented.

$$
\mathcal{L}_{L T}=\prod_{i=1}^{M} \mathcal{L}_{i} \quad \rightarrow \quad \log \left(\mathcal{L}_{L T}\right)=\sum_{i=1}^{M} \log \left(\mathcal{L}_{i}\right)
$$

Under the Poisson observation model used for the camera trap data, the expected values, $\lambda_{t j}$, explain the expected number of captures at trap $j$, on day $t$. The expected, or average, capture rate at a trap is given as:

$$
\lambda_{j t}=\lambda_{0} c_{j t} \sum_{i=1}^{M}\left[w_{i} d\left(x_{j}, s_{i}\right)\right]
$$

Here, $\lambda_{0}$ represents the maximum capture rate parameter for the Poisson observation model. This value can be interpreted as the expected capture rate when an animal's home range center is placed directly atop a trap. Note that $\lambda_{j t}=0$ if a trap is non-operational and $c_{j t}=0$, or if an individual is not predicted as part of the population, $w_{i}=0$. When this occurs, the corresponding 0 in the capture history will produce a likelihood value of 1 that is essentially ignored in subsequent calculations.

The likelihood of the captures histories, $y_{t j}$, given a set of expected values, $\lambda_{t j}$, is denoted as $\mathcal{L}_{t j}$ and is calculated by using the Poisson probability mass function.

$$
\mathcal{L}_{t j}=\operatorname{Poisson}\left(y_{t j} \mid \lambda_{t j}\right)=\frac{\lambda_{t j}^{y_{t j}} e^{\lambda_{t j}}}{y_{t j} !}
$$

The full conditional likelihood, $\mathcal{L}_{C T}$, is then calculated as the product of all the values of $\mathcal{L}_{t j}$. A log conversion of the likelihood is also implemented here. 


$$
\mathcal{L}_{C T}=\prod_{t=1}^{T} \prod_{j=1}^{J} \mathcal{L}_{t j} \quad \rightarrow \quad \log \left(\mathcal{L}_{C T}\right)=\sum_{t=1}^{T} \sum_{j=1}^{J} \log \left(\mathcal{L}_{t j}\right)
$$

\section{Estimation of the posterior parameter distributions}

The estimation of the posterior distributions of the parameters $\boldsymbol{s}_{i}, \sigma, w_{i}, N, \psi$, and $\lambda_{0} / \alpha_{0}$ is carried out using the Metropolis-Hasting Markov chain Monte Carlo algorithm. The algorithm can be thought of as an iterative guess-and-check type method. Different possible combinations of all the estimated parameters are chosen by randomly adjusting parameter values up or down according to a tuning distribution, which is typically a normal distribution. After a parameter value is adjusted up or down, its feasibility is compared against the previous parameter value using a ratio of the two full conditional likelihoods. Parameter estimates that have higher likelihood values than the previous estimate are more likely to be accepted, and the chains converge around these portions of the sample space. However, the chains never converge at a specific point since there is a random factor built into whether or not a parameter value is accepted. The end result is a set of posterior distributions, which give the relative likelihood of different possible values of the parameter. The basic structure of the algorithm is described on the following page. 
1. Load observed data:

I. Live trapping: $\boldsymbol{x}_{j}, K, y_{i j}$.

II. Camera trapping: $\boldsymbol{x}_{j}, T, y_{t j} c_{t j}$.

2. Choose parameters: $M, a, z, S$.

3. Choose tuning distribution parameters.

4. Choose desired prior distributions.

5. Choose starting values for parameters to be estimated: $\boldsymbol{s}_{i}, \sigma, w_{i}, N, \psi, \lambda_{0} / \alpha_{0}$.

6. Calculate: $\log \left(\mathcal{L}_{L T}\right)$ or $\log \left(\mathcal{L}_{C T}\right)$ using starting parameters.

7. Do the following for each parameter to be estimated: $\boldsymbol{s}_{i}, \sigma, w_{i}, N, \psi, \lambda_{0} / \alpha_{0}$.

I. Create a new candidate parameter estimate by randomly increasing or decreasing the current estimate according to its tuning distribution.

II. Using the new candidate parameters, calculate the candidate's (denoted with an asterisk *) full conditional $\log$ likelihood: $\log \left(\mathcal{L}_{L T}^{*}\right) \operatorname{or} \log \left(\mathcal{L}_{C T}^{*}\right)$.

III. Compare candidate full conditional likelihood to current full conditional likelihood by calculating:

a) With no prior: $r=\exp \left(\log \left(\mathcal{L}_{L T / C T}^{*}\right)-\log \left(\mathcal{L}_{L T / C T}\right)\right)$.

b) With prior:

i. Calculate prior likelihood by evaluating the prior distribution at the candidate $\left(\mathcal{L}_{P}^{*}\right)$ and current $\left(\mathcal{L}_{P}\right)$ parameter values.

$$
\text { ii. } \quad r=\exp \left(\log \left(\mathcal{L}_{L T / C T}^{*}\right)+\log \left(\mathcal{L}_{P}^{*}\right)-\log \left(\mathcal{L}_{L T / C T}\right)+\log \left(\mathcal{L}_{P}\right)\right) \text {. }
$$

IV. If $\mathrm{r}>1$, set $r=1$ and accept parameter candidate with probability $r$.

V. If candidate is accepted, set current parameter equal to the candidate value and update all parameters dependent on the new parameter value.

8. Once all parameters have been updated, record all current parameter values.

9. Repeat steps $7 \& 8$ for:

I. 10,000 'Burn in' iterations, which are thrown out.

II. Then 100,000 iterations which are saved.

10. Collect all 100,000 parameter estimates as posterior distributions of parameters. 


\section{Appendix C. Parameter Tables}

This appendix gives the exact parameter estimates for all five datasets: LT, CT, CT-LT, CTMix, and CTMx-LT. The values from these tables are displayed graphically throughout the paper as figures. The purpose of this appendix is to give transparency in the data and provide exact values for certain parameters that were difficult to display graphically.

\section{Appendix C1. Density and Abundance for CT \& CTMix}

Parameter estimates from the CT and CTMix datasets for abundance $(N)$ and density $(D)$. Estimates include mode, upper credible interval ("UCI"), and lower credible interval ("UCI"). Estimates for both detection functions are given without ("EP" and "HN") and with ("EP-SP" and "HN-SP") a prior on $\sigma$.

\begin{tabular}{|c|c|c|c|c|c|c|c|c|c|c|c|c|c|}
\hline & & \multicolumn{12}{|c|}{ Abundance $(\mathrm{N})$} \\
\hline & & \multicolumn{3}{|c|}{ EP } & \multicolumn{3}{|c|}{$\mathrm{HN}$} & \multicolumn{3}{|c|}{ EP-SP } & \multicolumn{3}{|c|}{ HN-SP } \\
\hline & & Mode & LCI & $\mathrm{UCI}$ & Mode & LCI & UCI & Mode & LCI & UCI & Mode & LCI & UCI \\
\hline \multirow[t]{10}{*}{$\mathrm{CT}$} & S1 & 75 & 13 & 75 & 75 & 11 & 75 & 21 & 10 & 71 & 75 & 14 & 75 \\
\hline & S2 & 12 & 6 & 68 & 10 & 6 & 69 & 14 & 7 & 58 & 16 & 7 & 70 \\
\hline & S3 & 75 & 7 & 75 & 75 & 8 & 75 & 9 & 4 & 50 & 11 & 5 & 63 \\
\hline & S4 & 75 & 10 & 75 & 75 & 12 & 75 & 14 & 7 & 68 & 19 & 9 & 73 \\
\hline & S5 & 1 & 1 & 44 & 1 & 1 & 46 & 3 & 1 & 22 & 4 & 1 & 25 \\
\hline & S6 & 6 & 4 & 68 & 6 & 4 & 69 & 5 & 3 & 14 & 5 & 4 & 14 \\
\hline & S7 & 2 & 2 & 62 & 5 & 2 & 68 & 2 & 2 & 12 & 5 & 2 & 18 \\
\hline & S8 & 5 & 3 & 71 & 3 & 3 & 70 & 6 & 3 & 33 & 8 & 3 & 34 \\
\hline & S9 & 75 & 10 & 75 & 75 & 9 & 75 & 11 & 4 & 63 & 10 & 4 & 63 \\
\hline & S10 & 15 & 5 & 72 & 75 & 4 & 73 & 9 & 4 & 31 & 5 & 4 & 36 \\
\hline \multirow[t]{5}{*}{ CTMix } & $\mathrm{S} 1+\mathrm{S} 2$ & 75 & 21 & 75 & 75 & 19 & 75 & 26 & 14 & 64 & 26 & 14 & 69 \\
\hline & $\mathrm{S} 3+\mathrm{S} 4$ & 75 & 22 & 75 & 75 & 16 & 75 & 75 & 14 & 75 & 75 & 14 & 75 \\
\hline & $\mathrm{S} 4+\mathrm{S} 5$ & 75 & 4 & 73 & 75 & 4 & 74 & 6 & 2 & 30 & 7 & 3 & 31 \\
\hline & $\mathrm{S} 5+\mathrm{S} 6$ & 75 & 14 & 75 & 19 & 13 & 75 & 7 & 4 & 24 & 10 & 4 & 26 \\
\hline & S6+S7 & 6 & 2 & 67 & 8 & 4 & 68 & 6 & 2 & 17 & 7 & 4 & 19 \\
\hline
\end{tabular}

\begin{tabular}{|c|c|c|c|c|c|c|c|c|c|c|c|}
\hline \multicolumn{12}{|c|}{ ensity (N/ha) } \\
\hline \multicolumn{3}{|c|}{ EP } & \multicolumn{3}{|c|}{$\mathrm{HN}$} & \multicolumn{3}{|c|}{ EP-SP } & \multicolumn{3}{|c|}{ HN-SP } \\
\hline Mode & $\mathrm{LCI}$ & UCI & Mode & LCI & $\mathrm{UCI}$ & Mode & LCI & $\mathrm{UCI}$ & Mode & LCI & UCI \\
\hline 24.1 & 4.18 & 24.1 & & 3.54 & 24.1 & .76 & 3.22 & 22.9 & 24.1 & 4.51 & 24.1 \\
\hline 4.60 & 2.30 & 26.0 & & 2.30 & 26.4 & 36 & 2.68 & 22.2 & 6.13 & 2.68 & 26.8 \\
\hline 44.7 & 4.17 & 44.7 & & 4.77 & 44.7 & 37 & 2.38 & 29.8 & 6.56 & 2.98 & 37.6 \\
\hline 45.7 & 6.10 & 45.7 & 45.7 & 7.32 & 45.7 & .54 & 4.27 & 41.5 & 11.6 & 5.49 & 44.5 \\
\hline 0.75 & 0.75 & 32.9 & 0 & 0.75 & 34.4 & 2.24 & 0.75 & 16.4 & 2.99 & 0.75 & 18.7 \\
\hline 4.25 & 2.83 & 48.2 & & & & & & 10.6 & & 83 & 9.92 \\
\hline 1.40 & 1.40 & 43.5 & & & & & & & & & 12.6 \\
\hline 3.51 & 2.10 & 49.8 & 2.10 & 2.10 & 49.1 & 4.21 & 2.10 & 23.2 & 5.61 & 2.10 & 23.9 \\
\hline 53.6 & 7.14 & 53.6 & 53.6 & 6.43 & 53.6 & 7.86 & 2.86 & 45.0 & 7.14 & 2.86 & 45.0 \\
\hline 10.7 & 3.57 & 51.4 & & 2.86 & 52.1 & & 2.86 & 22.1 & 3.57 & 2.86 & 25.7 \\
\hline 24.9 & 6.97 & & & & 24.9 & & 4.65 & 21.2 & & 4.65 & 22.9 \\
\hline 42.7 & 12.5 & 42.7 & & 9.11 & 42.7 & & 7.97 & 42.7 & 42.7 & 7.97 & 42.7 \\
\hline 44.0 & 3.52 & 44.0 & 44.0 & 2.35 & 43.4 & 3.52 & 1.17 & 17.6 & 4.10 & 1.76 & 18.2 \\
\hline 42.2 & 7.87 & 42.2 & 10.7 & 7.31 & 42.2 & & 2.25 & 13.5 & 5.62 & 2.25 & 14.6 \\
\hline 4.01 & 1.34 & 44.7 & 5.34 & 2.67 & 45.4 & 4.01 & 1.34 & 11.4 & 4.67 & 2.67 & 12.7 \\
\hline
\end{tabular}




\section{Appendix C2. Home Range Radius and Capture Rate CT \& CTMix}

Parameter estimates from the CT and CTMix datasets for home range size ("Sigma"; $\sigma$ ) and CT maximum capture rate ("Lambda 0"; $\lambda_{0}$ ). Estimates include mode, upper credible interval ("UCI"), and lower credible interval ("UCI"). Estimates for both detection functions are given without ("EP" and "HN") and with ("EP-SP" and "HN-SP") a prior on $\sigma$.

CT

\begin{tabular}{|c|c|c|c|c|c|c|c|c|c|c|c|c|}
\hline & \multicolumn{12}{|c|}{ Sigma (m) } \\
\hline & \multicolumn{3}{|c|}{ EP } & \multicolumn{3}{|c|}{$\mathrm{HN}$} & \multicolumn{3}{|c|}{ EP-SP } & \multicolumn{3}{|c|}{ HN-SP } \\
\hline & Mode & LCI & UCI & Mode & LCI & UCI & Mode & LCI & $\mathrm{UCI}$ & Mode & LCI & $\mathrm{UCI}$ \\
\hline S1 & 15.6 & 8.73 & 29.5 & 16.3 & 11.6 & 32.0 & 20.9 & 13.1 & 28.5 & 20.4 & 14.0 & 28.9 \\
\hline S2 & 22.3 & 10.1 & 38.2 & 24.7 & 13.3 & 34.7 & 20.3 & 15.0 & 32.5 & 20.3 & 15.2 & 31.2 \\
\hline S3 & 7.02 & 5.26 & 30.6 & 12.7 & 7.43 & 29.4 & 18.3 & 12.1 & 29.2 & 19.7 & 12.5 & 28.8 \\
\hline S4 & 13.1 & 7.41 & 30.8 & 13.1 & 8.39 & 26.4 & 19.3 & 11.7 & 28.3 & 17.5 & 12.1 & 27.4 \\
\hline S5 & 3.85 & 3.01 & 89.1 & 6.24 & 4.40 & 86.9 & 21.7 & 13.1 & 34.5 & 23.6 & 13.3 & 34.4 \\
\hline S6 & 7.53 & 1.80 & 15.9 & 5.32 & 3.39 & 19.2 & 15.8 & 11.2 & 23.4 & 17.6 & 12.4 & 22.3 \\
\hline S7 & 6.90 & 4.19 & 32.2 & 10.4 & 4.65 & 30.3 & 21.7 & 14.0 & 30.2 & 20.8 & 13.3 & 30.4 \\
\hline S8 & 5.27 & 3.51 & 28.0 & 10.3 & 7.24 & 33.6 & 18.9 & 12.1 & 29.2 & 19.9 & 13.1 & 31.3 \\
\hline S9 & 9.52 & 4.99 & 22.6 & 10.5 & 6.31 & 24.0 & 17.0 & 10.3 & 27.1 & 17.3 & 11.1 & 26.9 \\
\hline $\mathrm{S} 10$ & 7.38 & 4.84 & 18.1 & 7.14 & 5.72 & 20.5 & 14.1 & 10.6 & 22.8 & 15.3 & 10.9 & 23.1 \\
\hline
\end{tabular}

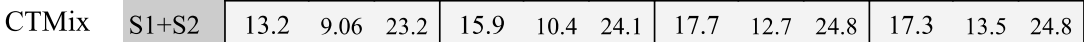
\begin{tabular}{l|lll|lll|lll|lll|l}
$\mathrm{S} 3+\mathrm{S} 4$ & 11.2 & 7.14 & 21.8 & 11.0 & 7.84 & 24.5 & 15.8 & 10.5 & 26.4 & 16.0 & 10.5 & 26.0
\end{tabular} \begin{tabular}{l|lll|lll|lll|lll}
$\mathrm{S} 4+\mathrm{S} 5$ & 4.84 & 2.21 & 23.8 & 6.54 & 3.96 & 28.8 & 20.0 & 11.2 & 28.3 & 17.8 & 11.4 & 28.3
\end{tabular}

\begin{tabular}{l|lll|lll|lll|lll|}
$\mathrm{S} 5+\mathrm{S} 6$ & 3.17 & 1.70 & 8.82 & 4.38 & 1.54 & 9.36 & 14.5 & 8.96 & 24.0 & 13.5 & 8.16 & 21.0 \\
\hline
\end{tabular}

\begin{tabular}{l|lll|lll|lll|llll}
$\mathrm{S} 6+\mathrm{S} 7$ & 6.46 & 3.12 & 28.0 & 8.53 & 4.83 & 23.2 & 18.8 & 12.2 & 28.0 & 19.8 & 13.7 & 25.5 \\
\cline { 2 - 8 }
\end{tabular}
Lambda 0 (Capts/Cam/Day)

\begin{tabular}{|ccc|ccc|ccc|ccc|}
\hline \multicolumn{6}{|c|}{} & EP & \multicolumn{4}{|c|}{ HN } & \multicolumn{3}{|c|}{ EP-SP } & \multicolumn{3}{|c|}{ HN-SP } \\
Mode & LCI & UCI & Mode & LCI & UCI & Mode & LCI & UCI & Mode & LCI & UCI \\
\hline 1.44 & 0.57 & 10.1 & 0.76 & 0.37 & 1.45 & 1.25 & 0.58 & 5.97 & 0.72 & 0.34 & 1.36 \\
1.35 & 0.55 & 5.80 & 0.62 & 0.28 & 1.39 & 1.42 & 0.63 & 4.18 & 0.59 & 0.26 & 1.34 \\
0.36 & 0.05 & 3.54 & 0.18 & 0.06 & 0.57 & 0.40 & 0.10 & 2.01 & 0.17 & 0.05 & 0.52 \\
0.52 & 0.15 & 2.37 & 0.24 & 0.10 & 0.61 & 0.52 & 0.17 & 1.66 & 0.23 & 0.10 & 0.54 \\
0.31 & 0.01 & 4.47 & 0.12 & 0.01 & 0.61 & 0.41 & 0.02 & 1.29 & 0.14 & 0.01 & 0.38 \\
1.36 & 0.67 & 102 & 0.90 & 0.24 & 9.16 & 1.31 & 0.50 & 6.04 & 0.85 & 0.29 & 2.00 \\
1.16 & 0.22 & 12.4 & 0.51 & 0.11 & 2.07 & 0.94 & 0.32 & 6.56 & 0.49 & 0.15 & 1.15 \\
1.11 & 0.16 & 61.1 & 0.55 & 0.18 & 1.22 & 0.82 & 0.24 & 6.42 & 0.48 & 0.17 & 1.17 \\
0.63 & 0.15 & 8.34 & 0.31 & 0.10 & 0.91 & 0.57 & 0.14 & 2.99 & 0.21 & 0.09 & 0.83 \\
1.91 & 0.62 & 15.3 & 0.89 & 0.34 & 3.15 & 1.77 & 0.59 & 7.89 & 0.74 & 0.37 & 2.68 \\
\hline
\end{tabular}

\begin{tabular}{|lll|lll|lll|lll|}
\hline 2.13 & 0.99 & 8.47 & 0.97 & 0.51 & 2.00 & 1.91 & 0.89 & 5.08 & 1.01 & 0.50 & 1.83 \\
0.91 & 0.33 & 2.50 & 0.46 & 0.17 & 0.88 & 0.64 & 0.25 & 1.82 & 0.33 & 0.14 & 0.71
\end{tabular} \begin{tabular}{lll|lll|lll|llll}
0.91 & 0.33 & 2.50 & 0.46 & 0.17 & 0.84 & 0.64 & 0.25 & 1.82 & 0.33 & 0.14 & 0.71
\end{tabular} \begin{tabular}{lll|llll|lllllll}
0.58 & 0.07 & 38.2 & 0.25 & 0.02 & 0.76 & 0.42 & 0.08 & 1.38 & 0.19 & 0.03 & 0.49
\end{tabular} \begin{tabular}{lll|lll|llll|lll}
2.60 & 0.36 & 35.5 & 0.91 & 0.20 & 119 & 0.81 & 0.30 & 2.82 & 0.49 & 0.17 & 1.40
\end{tabular} \begin{tabular}{lll|lllllllllll}
1.30 & 0.41 & 12.6 & 0.56 & 0.22 & 1.69 & 1.75 & 0.45 & 5.02 & 0.65 & 0.20 & 1.32 \\
\hline
\end{tabular} 


\section{Appendix C3. All Parameters for CT-LT, CTMx-LT \& LT}

Parameter estimates from the CT-LT, CTMx-LT, and LT datasets for abundance $(N)$, density $(D)$, home range size ("Sigma"; $\sigma$ ), LT capture rate ("Alpha 0"; $\alpha_{0}$ ), and maximum CT capture rate ("Lambda $0 " ; \lambda_{0}$ ). Estimates include mode, upper credible interval ("UCI"), and lower credible interval ("UCI"). Estimates for both detection functions are given ("EP" and "HN").

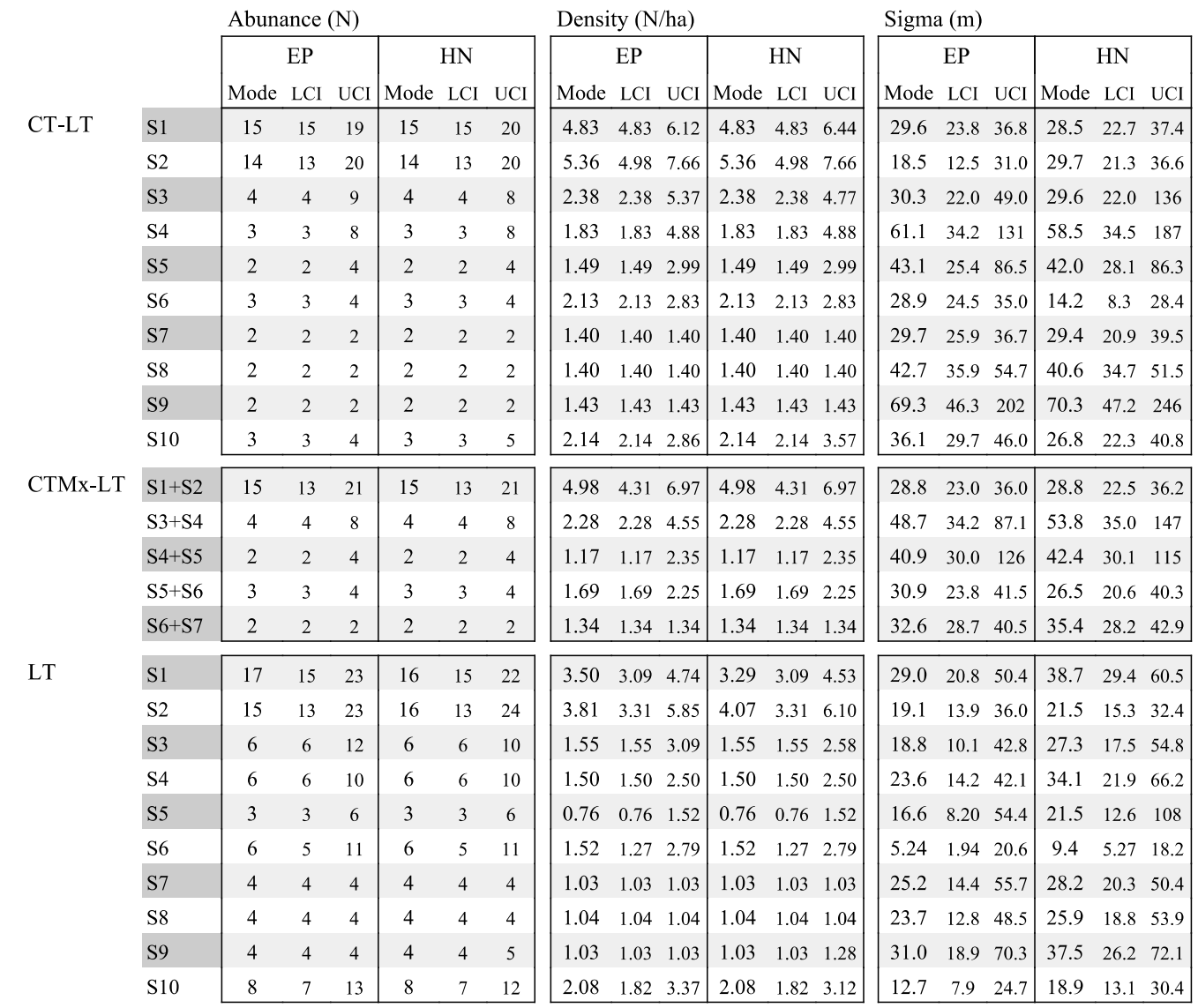

Alpha 0
\begin{tabular}{|ccc|ccc|}
\hline \multicolumn{3}{|c|}{ EP } & \multicolumn{3}{|c|}{ HN } \\
Mode & LCI & UCI & Mode & LCI & UCI \\
\hline-0.08 & -1.02 & 0.70 & 0.03 & -0.92 & 0.89 \\
1.24 & 0.00 & 2.37 & -0.14 & -1.11 & 0.82 \\
-0.66 & -2.36 & 1.00 & -1.76 & -3.90 & 0.13 \\
-0.90 & -3.28 & 0.86 & -2.35 & -4.54 & -0.45 \\
-0.71 & -3.03 & 3.16 & -0.66 & -3.38 & 2.69 \\
0.54 & -1.34 & 2.63 & 3.02 & 0.86 & 5.70 \\
5.50 & -0.53 & 211 & 49.6 & 2.59 & 116 \\
34.5 & -0.53 & 200 & 67.0 & 2.77 & 264 \\
164 & -0.66 & 203 & 31.0 & -1.24 & 183 \\
0.84 & -1.32 & 2.84 & 0.12 & -1.89 & 2.03 \\
\hline-0.51 & -1.43 & 0.33 & -0.31 & -1.27 & 0.52 \\
-1.72 & -3.40 & -0.05 & -2.13 & -4.22 & -0.58 \\
-1.44 & -4.07 & 2.07 & -1.40 & -4.07 & 2.15 \\
0.39 & -1.78 & 2.35 & 1.06 & -1.01 & 3.34 \\
63.8 & 15.3 & 665 & 144 & 17.5 & 311 \\
\hline 0.20 & -0.84 & 1.44 & -0.97 & -2.10 & -0.05 \\
1.02 & -0.43 & 2.39 & -0.17 & -1.25 & 1.04 \\
1.27 & -0.81 & 3.45 & -0.09 & -1.78 & 1.60 \\
1.45 & -0.74 & 3.73 & -0.45 & -2.26 & 1.88 \\
1.49 & -1.27 & 5.07 & 0.72 & -2.53 & 3.78 \\
3.95 & 0.36 & 11.7 & 2.18 & -0.30 & 7.31 \\
22.0 & 3.97 & 269 & 106 & 32.6 & 272 \\
5.19 & 1.90 & 164 & 508 & 184 & 564 \\
2.76 & 0.08 & 6.85 & 1.21 & -1.04 & 5.34 \\
2.41 & 0.66 & 4.57 & 0.80 & -0.45 & 2.75 \\
\hline
\end{tabular}

\begin{tabular}{|c|c|c|c|c|c|}
\hline & EP & & & $\mathrm{HN}$ & \\
\hline Mode & LCI & $\mathrm{UCI}$ & Mode & LCI & $\mathrm{U}$ \\
\hline 0.96 & 0.62 & 1.84 & 1.10 & 0.67 & 2 \\
\hline 3.17 & 1.46 & 13.5 & 1.22 & 0.69 & \\
\hline 0.35 & 0.13 & 0.86 & 0.10 & 0.02 & \\
\hline 0.64 & 0.11 & 1.82 & 0.25 & 0.04 & \\
\hline 0.14 & 0.03 & 0.38 & 0.10 & 0.03 & \\
\hline 0.58 & 0.36 & 0.83 & 3.87 & 0.94 & \\
\hline 0.59 & 0.38 & 0.98 & 1.12 & 0.50 & 3 \\
\hline 0.63 & 0.40 & 0.91 & $\mid 0.49$ & 0.31 & \\
\hline 0.16 & 0.08 & 0.46 & 0.18 & 0.12 & \\
\hline 1.51 & 0.90 & 2.47 & 1.32 & 0.68 & \\
\hline 1.21 & 0.72 & 2.12 & 1.51 & 0.86 & \\
\hline 0.39 & 0.15 & 0.73 & 0.21 & 0.06 & \\
\hline 0.10 & 0.02 & 0.20 & 0.09 & 0.03 & \\
\hline 0.29 & 0.14 & 0.53 & 0.61 & 0.26 & \\
\hline 0.44 & 0.29 & 0.60 & 0.77 & 0.49 & \\
\hline
\end{tabular}


Appendix C4. Bayesian p-values for CT, CTMix, CT-LT, CTMx-LT \& LT

Bayesian p-values calculated from the CT-LT, CTMx-LT, and LT datasets. Estimates for both detection functions are given without (EP and HN) and with (EP-SP and HN-SP) a prior on $\sigma$. The CT-LT and CTMx-LT datasets give separate Bayesian pvalues for the live trapping (LT) and camera trapping (CT) data separately.

CT

\begin{tabular}{l|c|c|c|c|}
\cline { 2 - 5 } \multicolumn{1}{c|}{} & EP & HN & EP-SP & HN-SP \\
\cline { 2 - 5 } S1 & 0.001 & 0.001 & 0.001 & 0.001 \\
S2 & 0.569 & 0.498 & 0.578 & 0.525 \\
S3 & 0.432 & 0.451 & 0.365 & 0.381 \\
S4 & 0.410 & 0.462 & 0.357 & 0.384 \\
S5 & 0.300 & 0.271 & 0.369 & 0.391 \\
S6 & 0.510 & 0.502 & 0.405 & 0.457 \\
S7 & 0.506 & 0.499 & 0.439 & 0.443 \\
S8 & 0.407 & 0.390 & 0.276 & 0.358 \\
S9 & 0.469 & 0.477 & 0.298 & 0.385 \\
S10 & 0.152 & 0.133 & 0.097 & 0.107 \\
\hline
\end{tabular}

CTMix

\begin{tabular}{l|l|l|l|l|}
\cline { 2 - 5 } S1+S2 & 0.519 & 0.496 & 0.446 & 0.450 \\
S3+S4 & 0.475 & 0.393 & 0.276 & 0.226 \\
S4+S5 & 0.457 & 0.389 & 0.225 & 0.182 \\
S5+S6 & 0.504 & 0.541 & 0.156 & 0.191 \\
S6+S7 & 0.388 & 0.323 & 0.201 & 0.143 \\
\cline { 2 - 5 } & \multicolumn{2}{|c}{}
\end{tabular}

CT-LT

CTMx-LT

\begin{tabular}{|c|c|c|c|c|}
\hline & EP-LT & HN-LT & EP-CT & $\mathrm{HN}-\mathrm{CT}$ \\
\hline S1 & 0.233 & 0.253 & 0.003 & 0.003 \\
\hline S2 & 0.345 & 0.320 & 0.367 & 0.357 \\
\hline S3 & 0.134 & 0.117 & 0.067 & 0.012 \\
\hline S4 & 0.333 & 0.304 & 0.001 & 0.000 \\
\hline S5 & 0.352 & 0.334 & 0.213 & 0.151 \\
\hline S6 & 0.356 & 0.276 & 0.001 & 0.068 \\
\hline S7 & 0.663 & 0.479 & 0.168 & 0.308 \\
\hline S8 & 0.637 & 0.493 & 0.000 & 0.000 \\
\hline S9 & 0.337 & 0.411 & 0.000 & 0.000 \\
\hline S10 & 0.018 & 0.003 & 0.000 & 0.001 \\
\hline $\mathrm{S} 1+\mathrm{S} 2$ & 0.444 & 0.409 & 0.018 & 0.024 \\
\hline $\mathrm{S} 3+\mathrm{S} 4$ & 0.155 & 0.150 & 0.003 & 0.001 \\
\hline $\mathrm{S} 4+\mathrm{S} 5$ & 0.361 & 0.347 & 0.002 & 0.003 \\
\hline S5+S6 & 0.542 & 0.612 & 0.000 & 0.003 \\
\hline $\mathrm{S} 6+\mathrm{S} 7$ & 0.949 & 0.798 & 0.000 & 0.014 \\
\hline
\end{tabular}

LT

\begin{tabular}{l|c|c|}
\cline { 2 - 3 } \multicolumn{1}{c|}{} & EP & HN \\
\cline { 2 - 3 } S1 & 0.454 & 0.278 \\
S2 & 0.522 & 0.489 \\
S3 & 0.225 & 0.052 \\
S4 & 0.316 & 0.126 \\
S5 & 0.391 & 0.327 \\
S6 & 0.541 & 0.549 \\
S7 & 0.547 & 0.510 \\
S8 & 0.317 & 0.158 \\
S9 & 0.298 & 0.156 \\
S10 & 0.256 & 0.081 \\
\cline { 2 - 3 }
\end{tabular}




\section{Appendix D. Home Range Center Estimates}

This appendix gives the home range center estimates for three datasets: LT, CT-LT, and CTMx-LT. For every session of data, a graphical representation is given for the locations of home range centers $\left(\boldsymbol{s}_{i}\right)$ and the polygon used as the state space $(S)$. Animals with known identities are listed with colored dots, the color of which corresponds to the tag border color used in Appendix A. Individuals with unknown identities are listed with white dots. For each session of data, home range center estimates for the EP and HN detection functions are given.

In addition to the home range center information, the data are plotted against satellite images obtained from imagery dated 25 August, 2013 in Google Earth (C) Google Earth 2016). The images were tiled together and georeferenced, and the resulting image was down sampled and annotated with the points in MATLAB. Data are displayed in UTM Zone 10S, on the NAD 1983 Datum. 


\section{LT Session S1 Home Range Center Estimates}
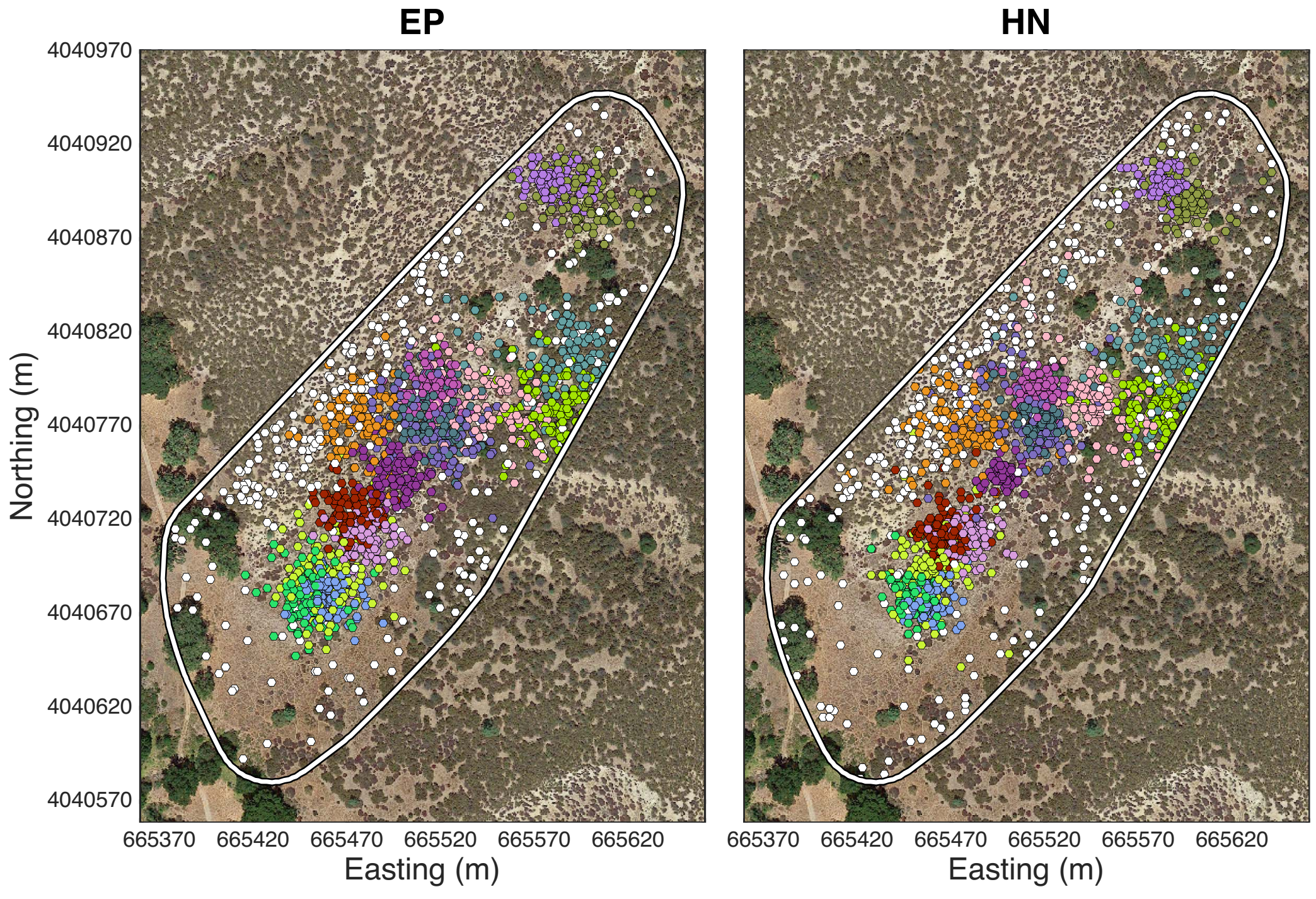


\section{LT Session S2 Home Range Center Estimates}
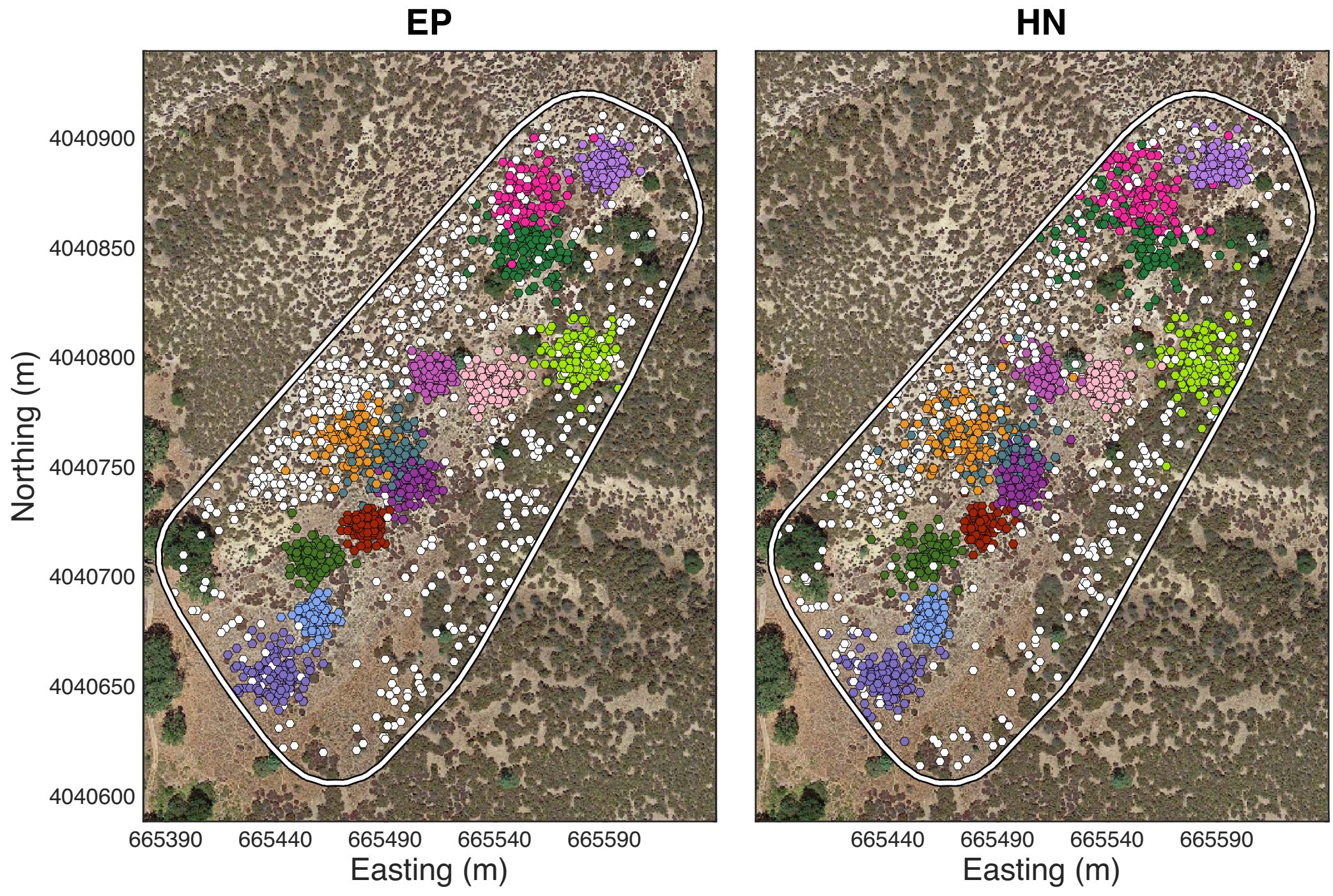


\section{LT Session S3 Home Range Center Estimates}

EP

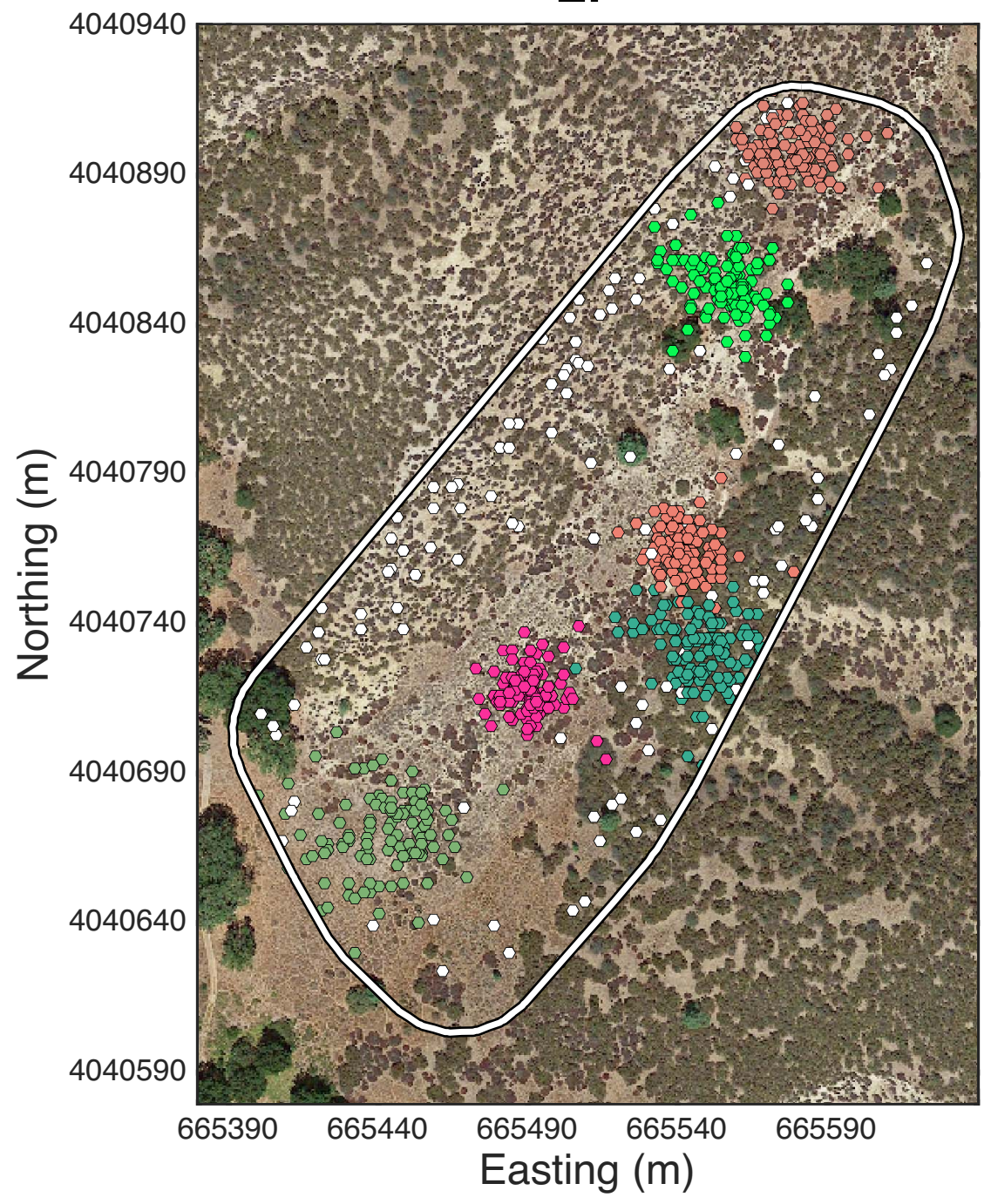

HN

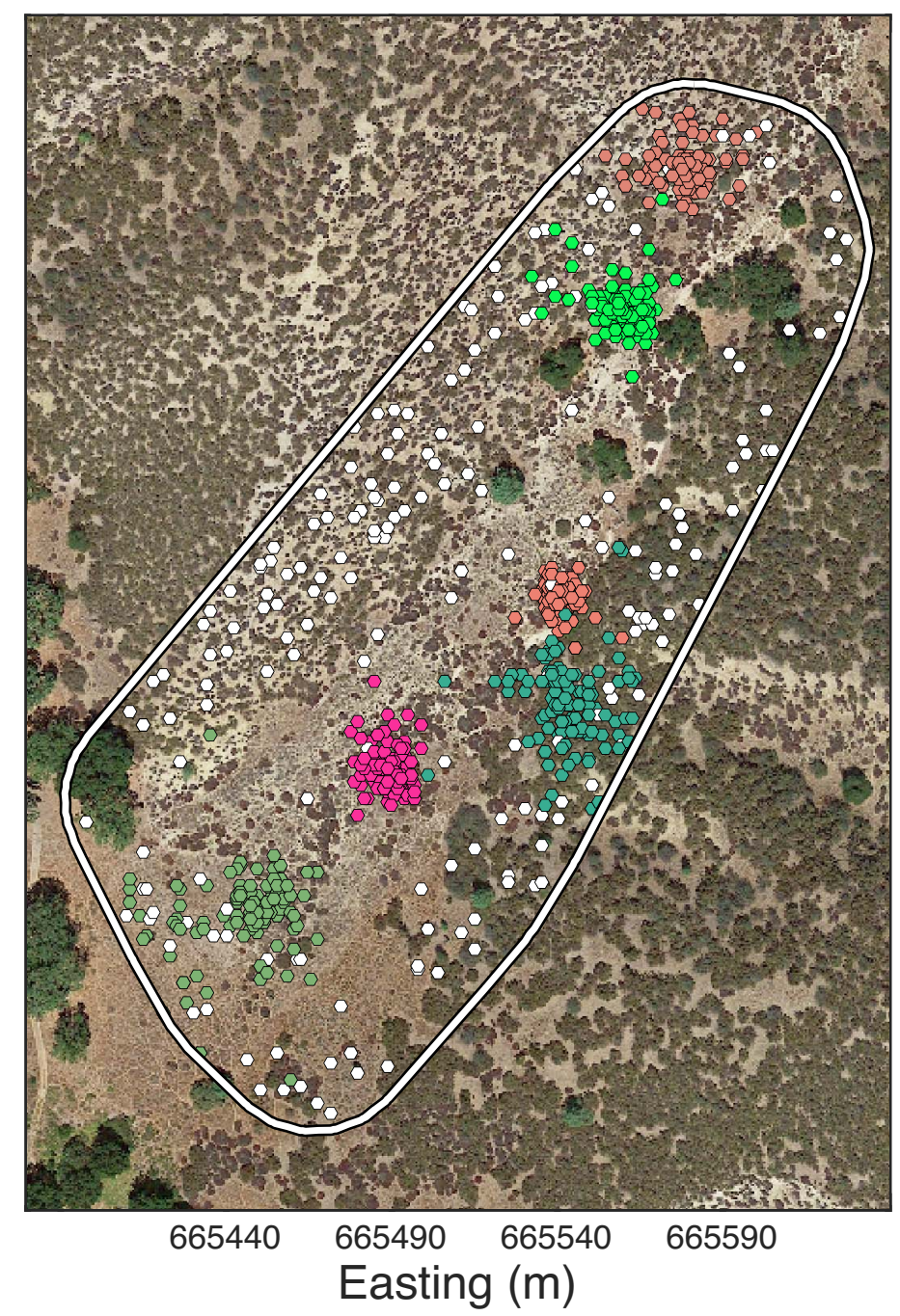


LT Session S4 Home Range Center Estimates EP

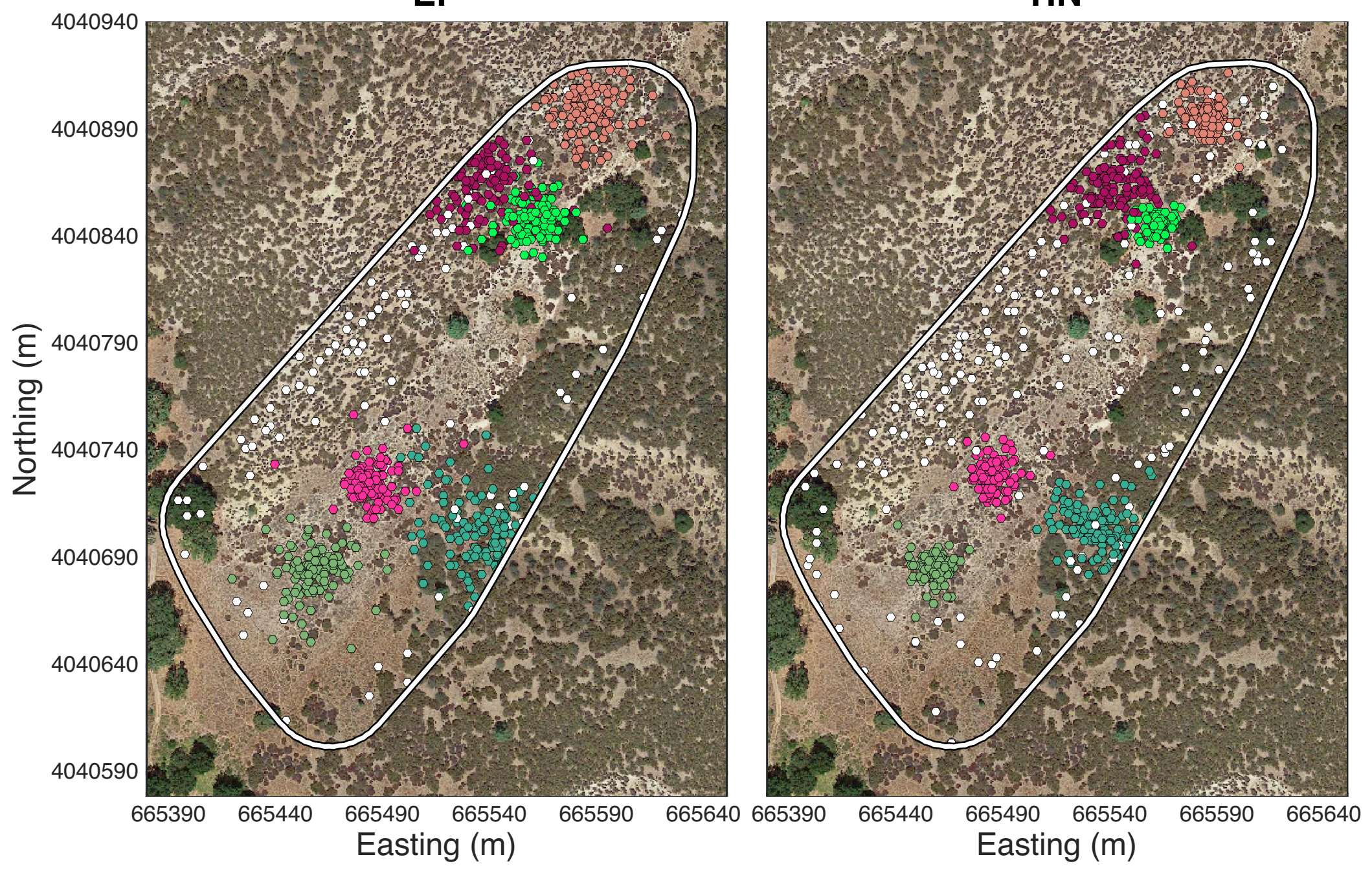




\section{LT Session S5 Home Range Center Estimates}

EP

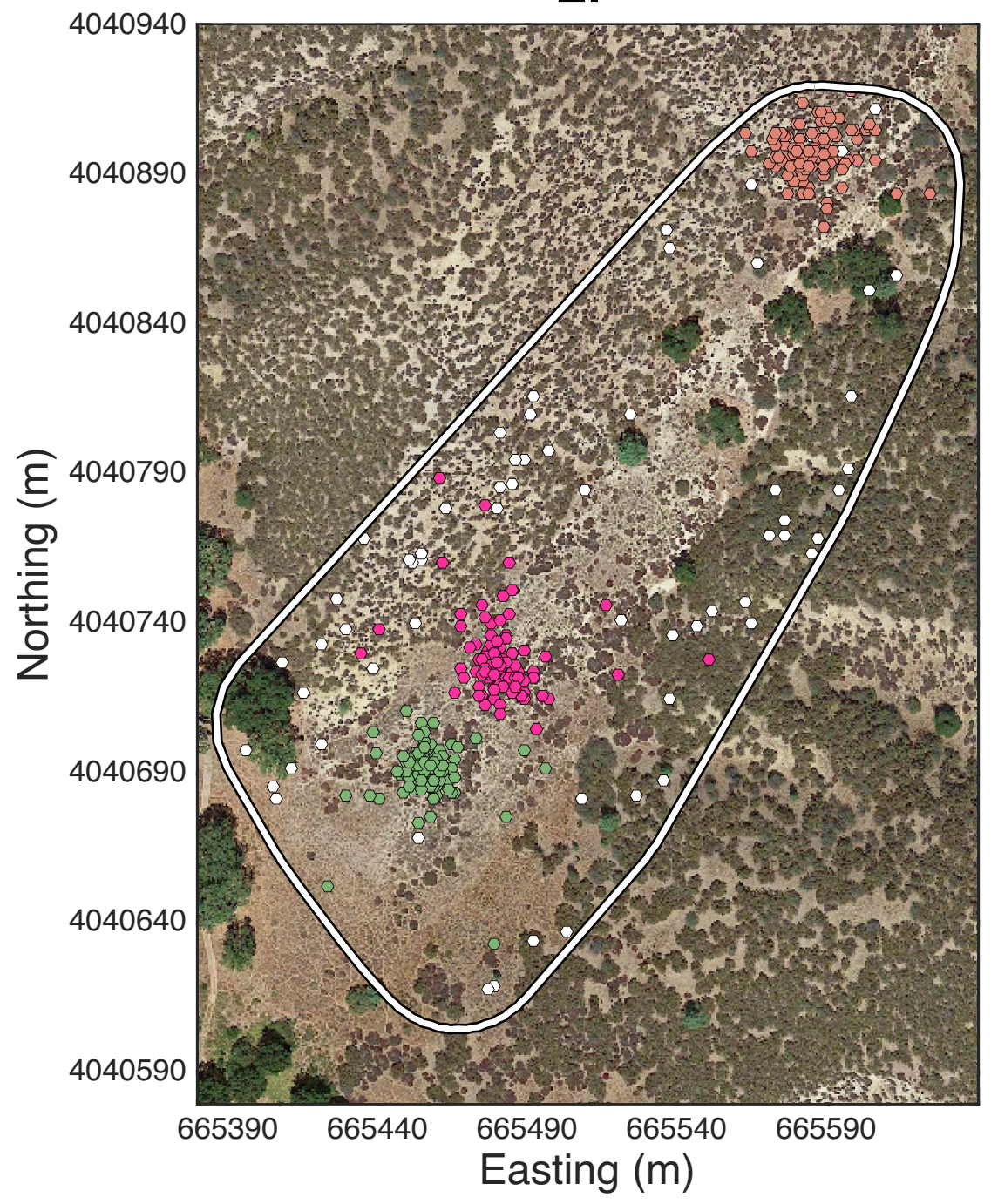

HN

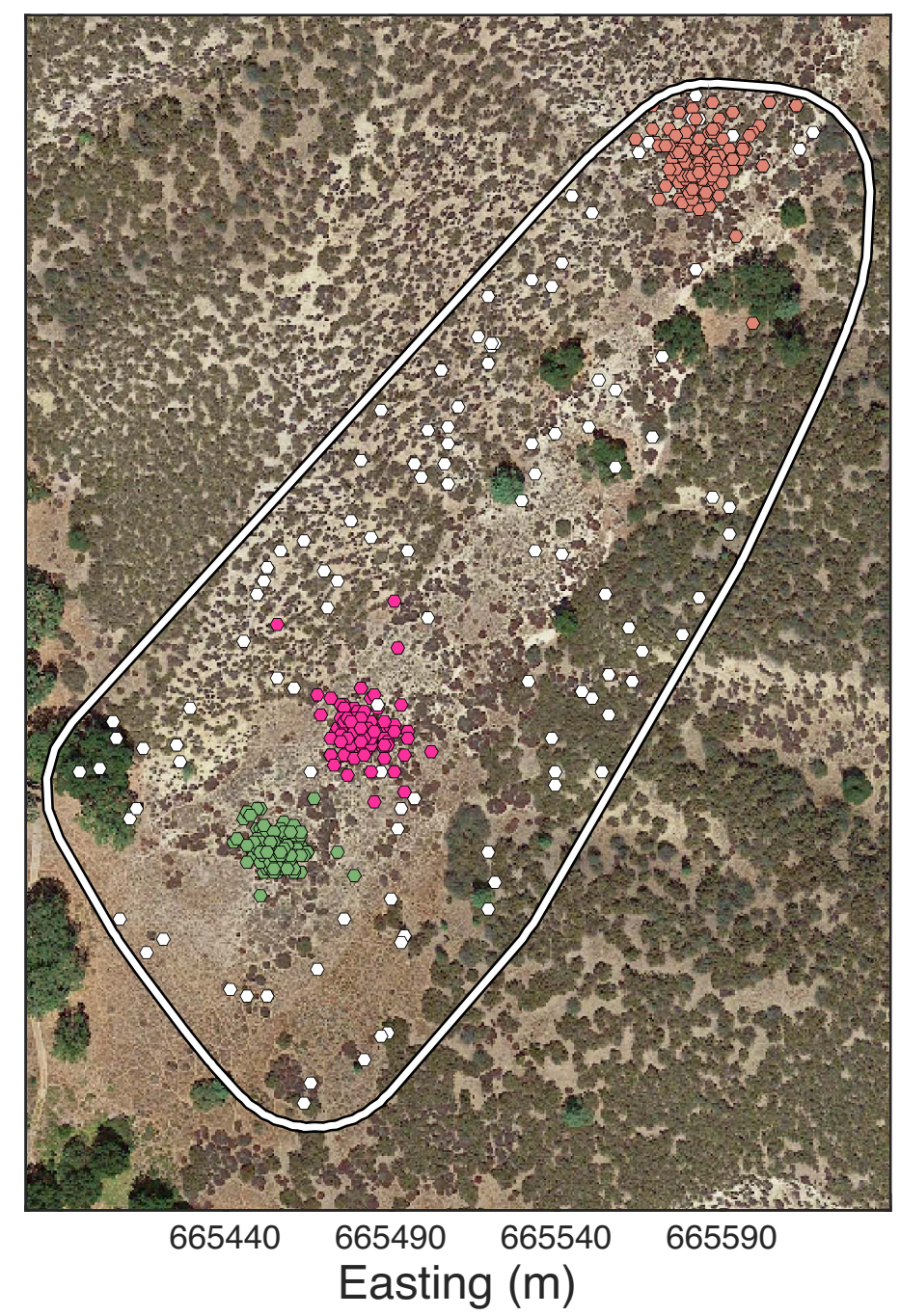




\section{LT Session S6 Home Range Center Estimates}

EP

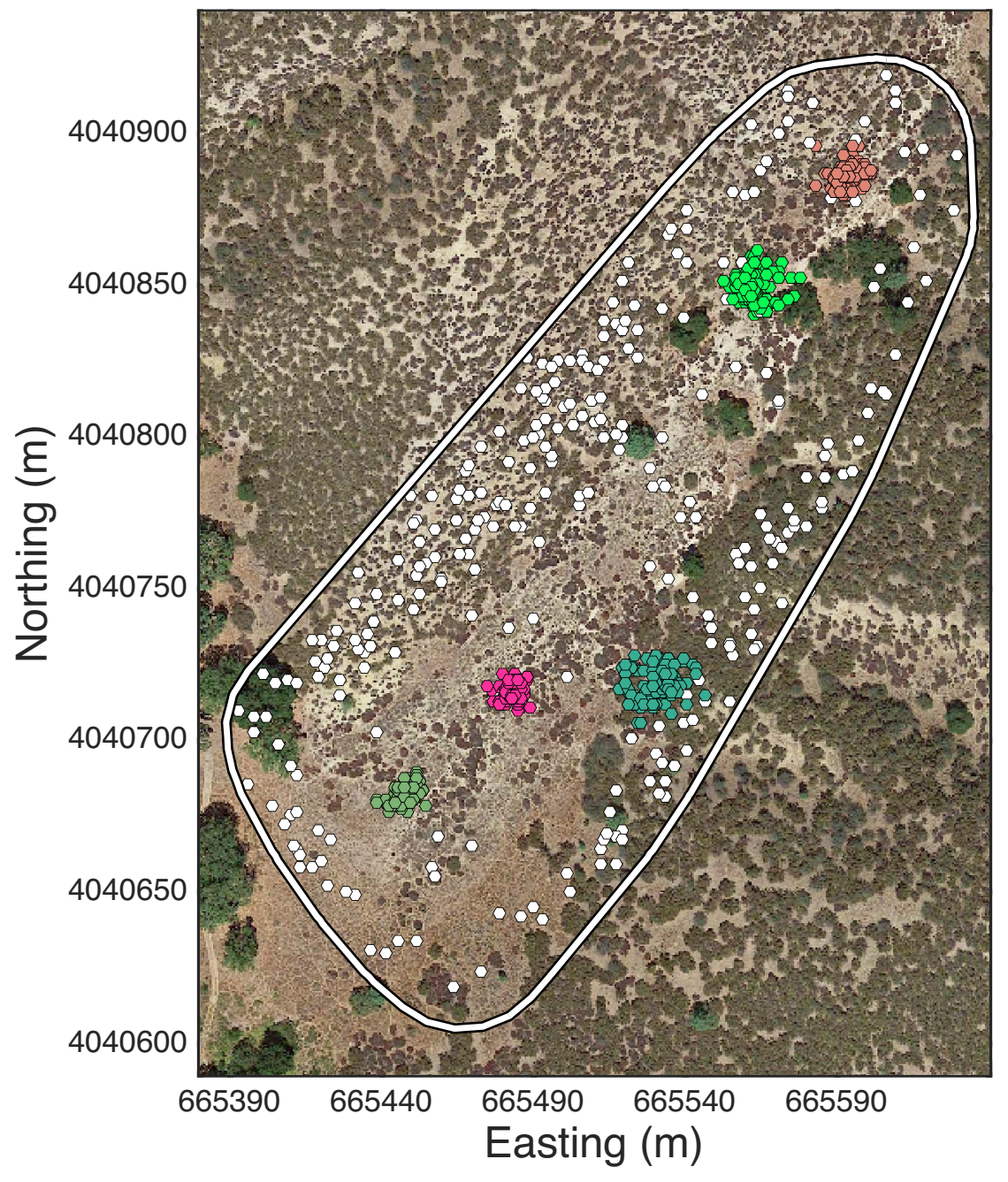

HN

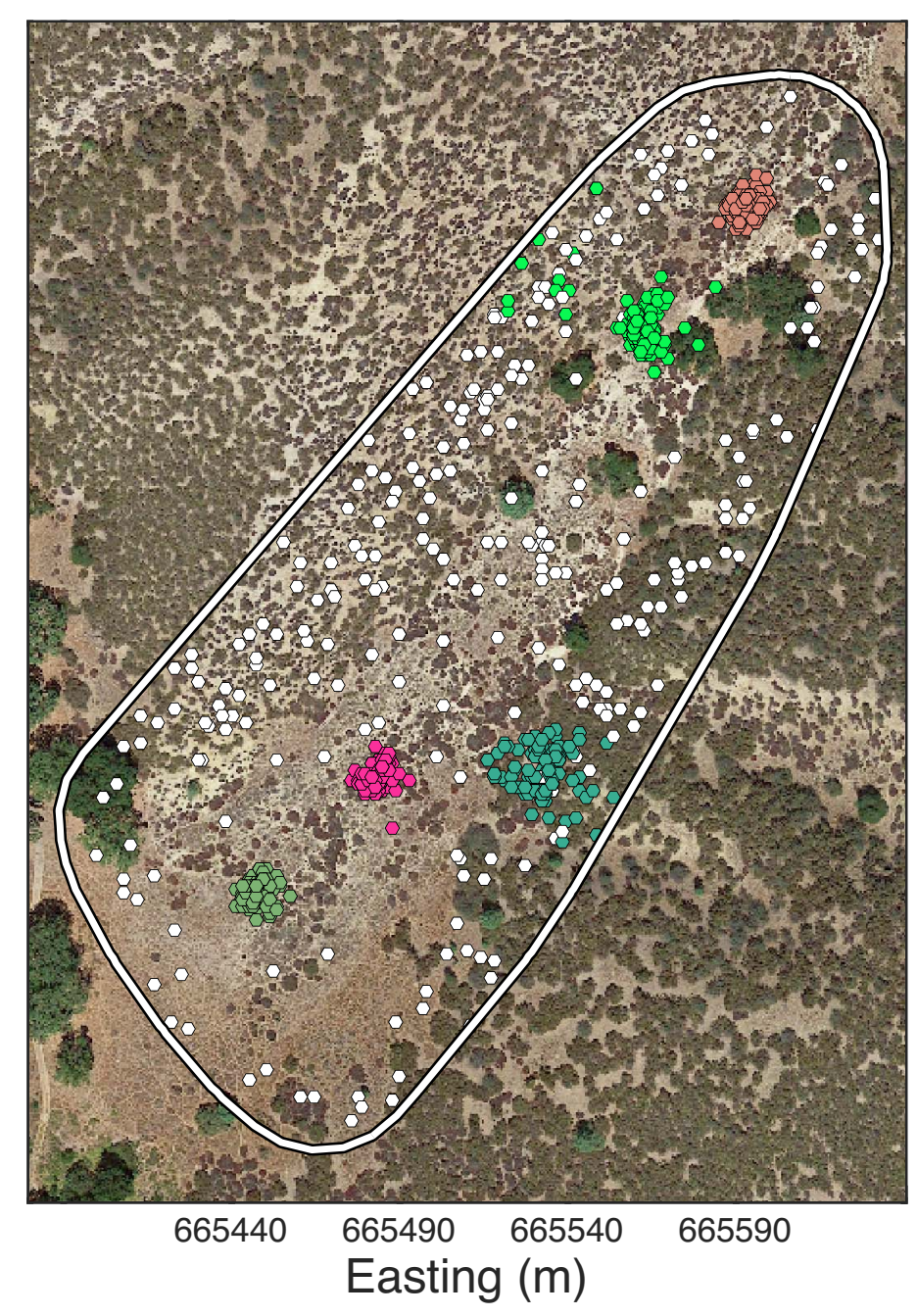




\section{LT Session S7 Home Range Center Estimates}

EP

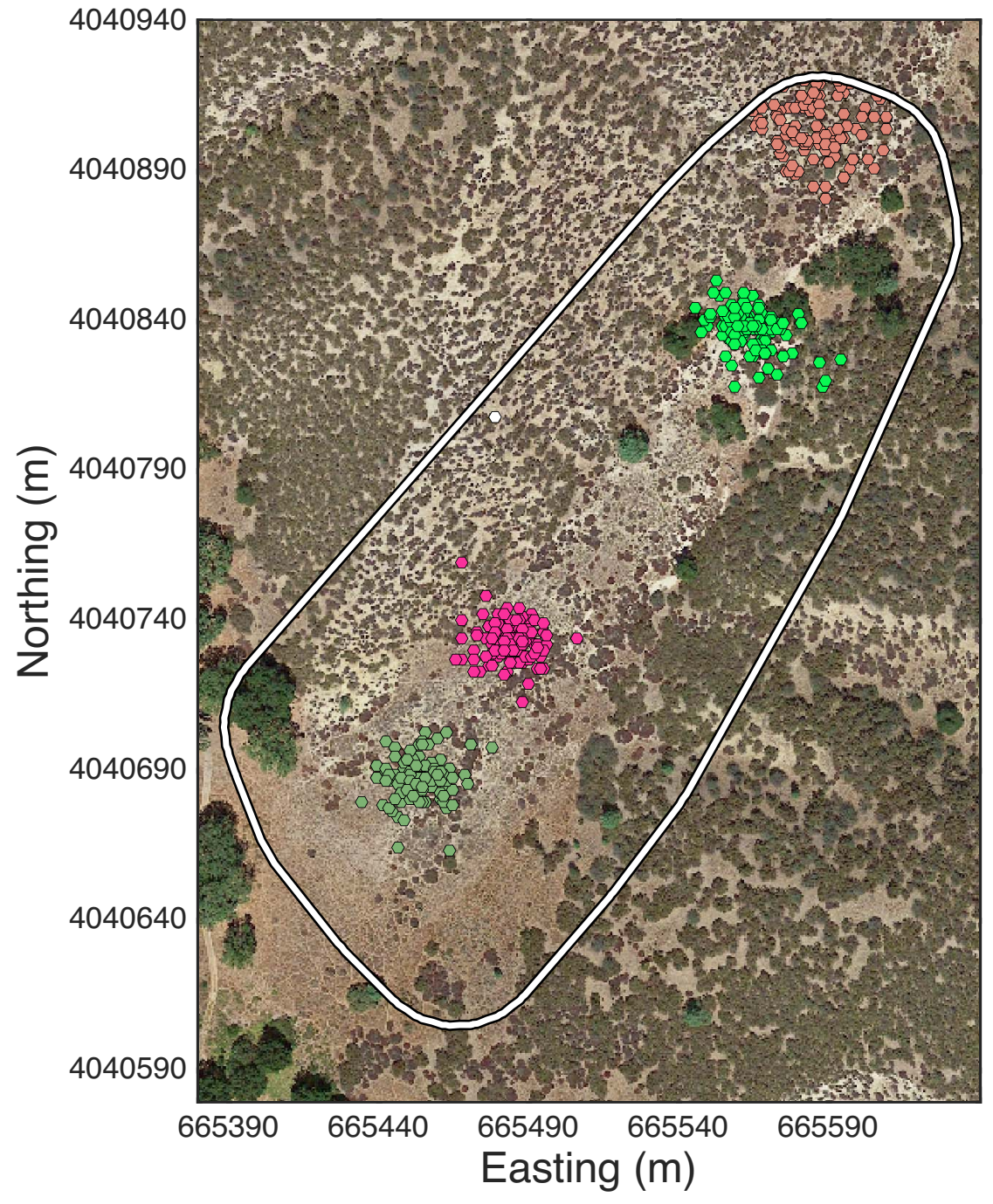

HN

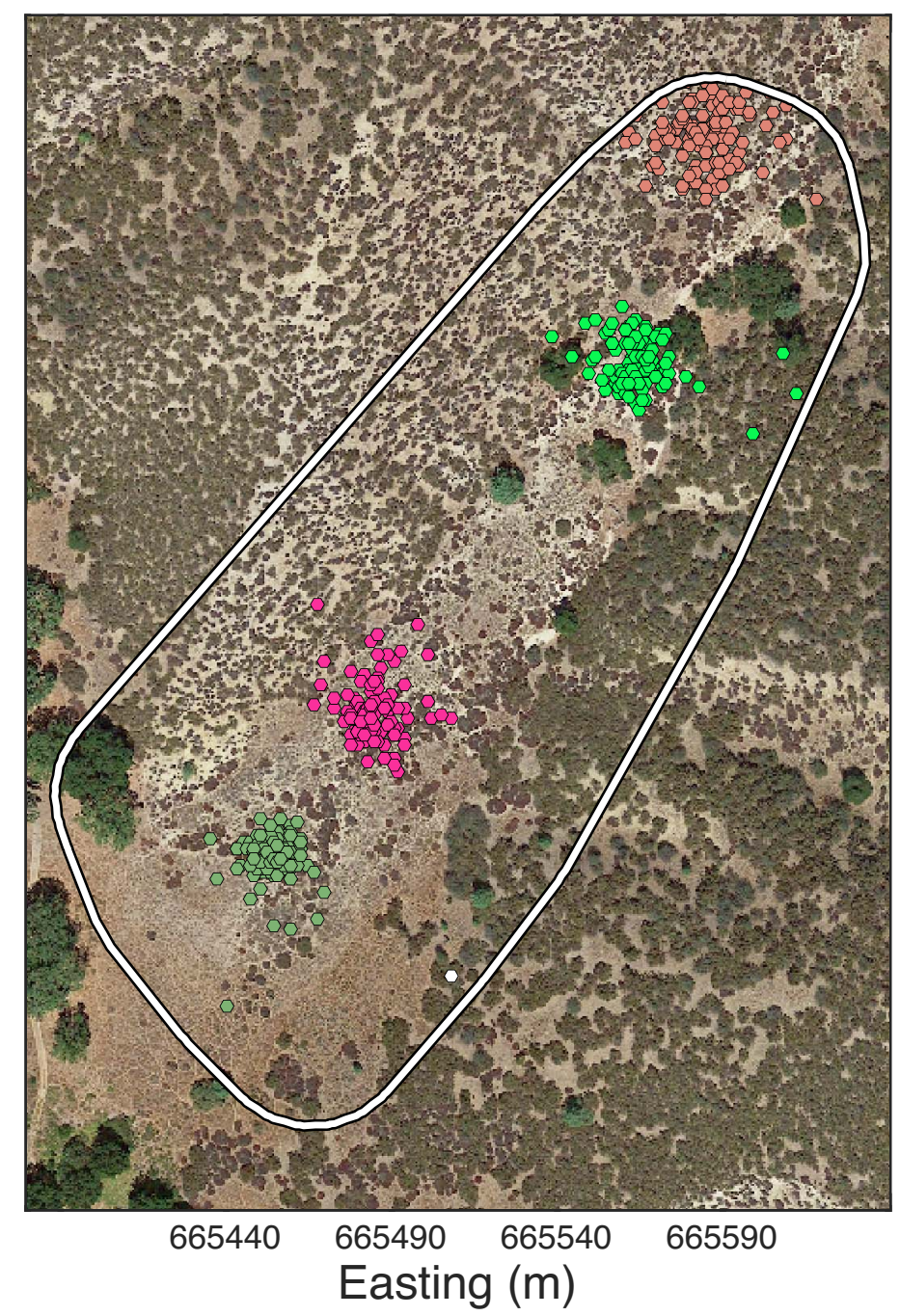




\section{LT Session S8 Home Range Center Estimates}

EP

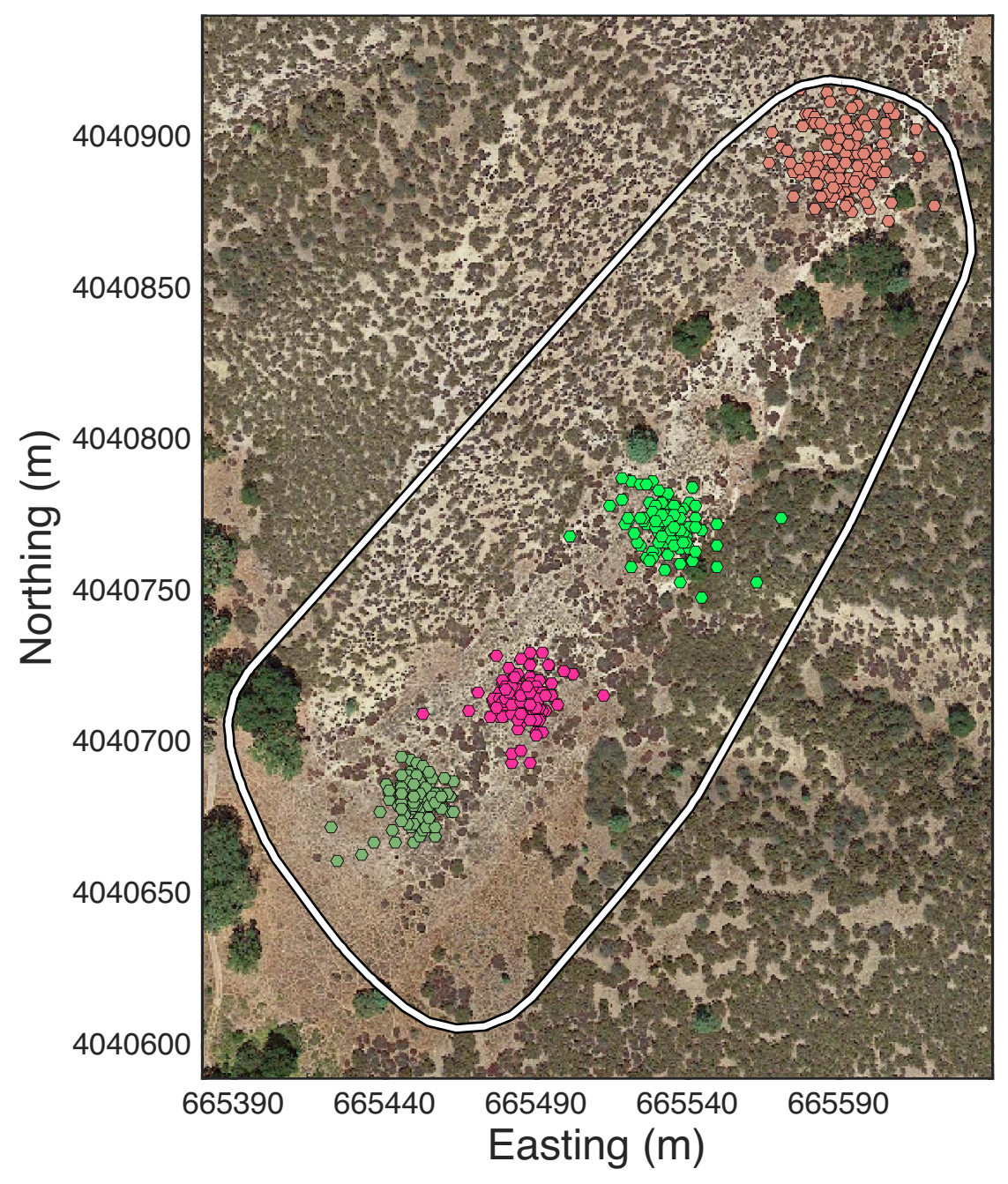

HN

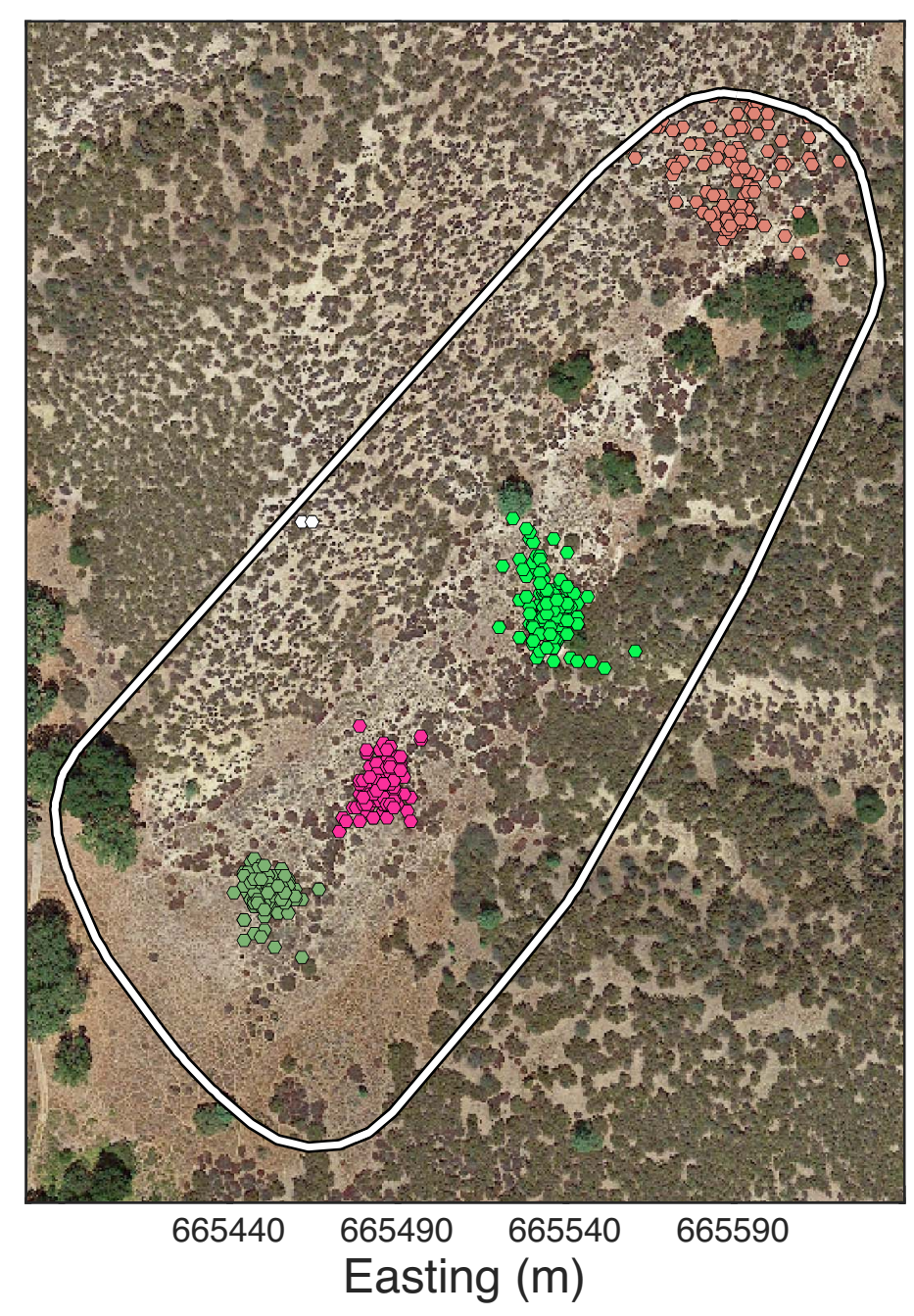




\section{LT Session S9 Home Range Center Estimates}

EP

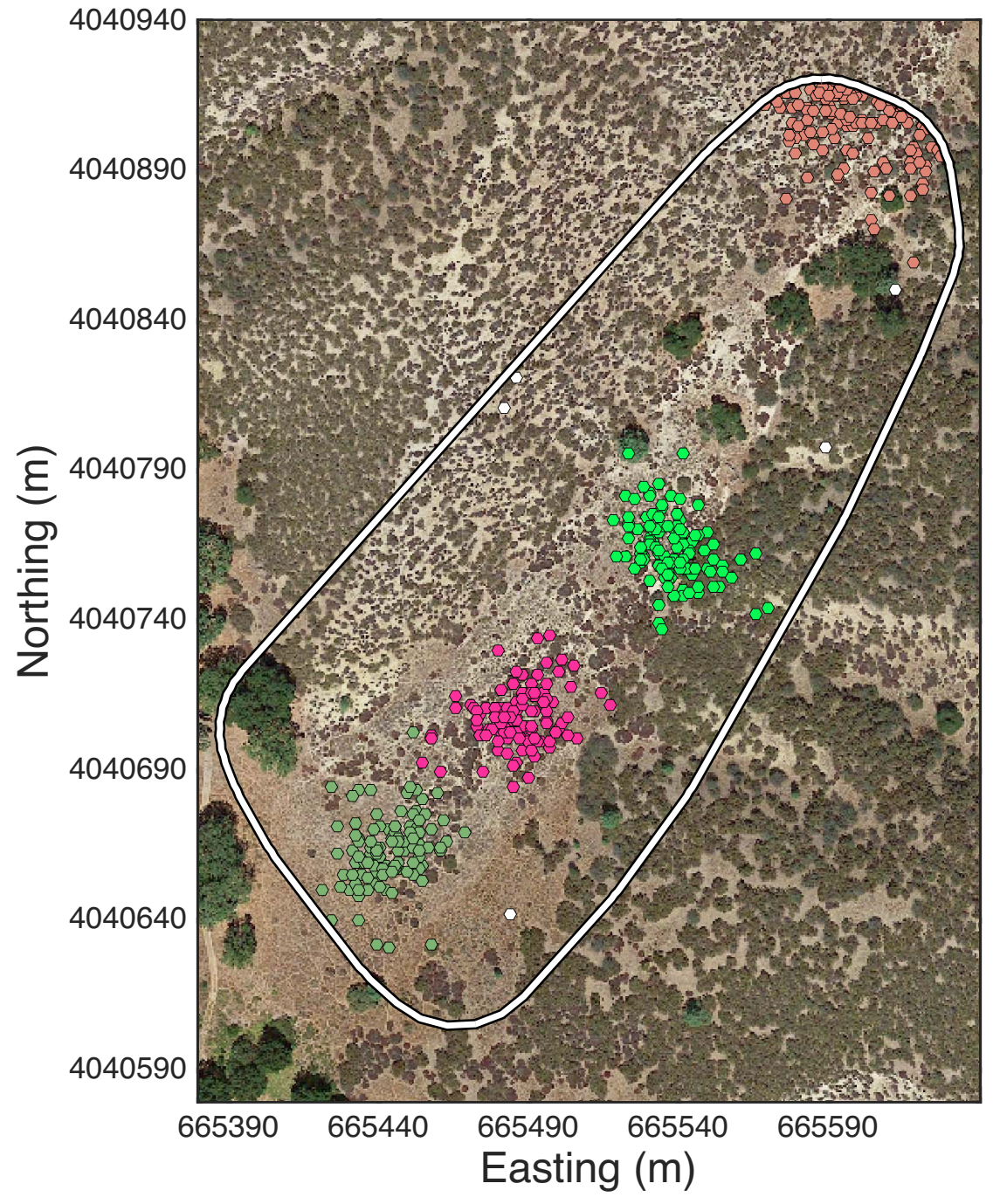

HN

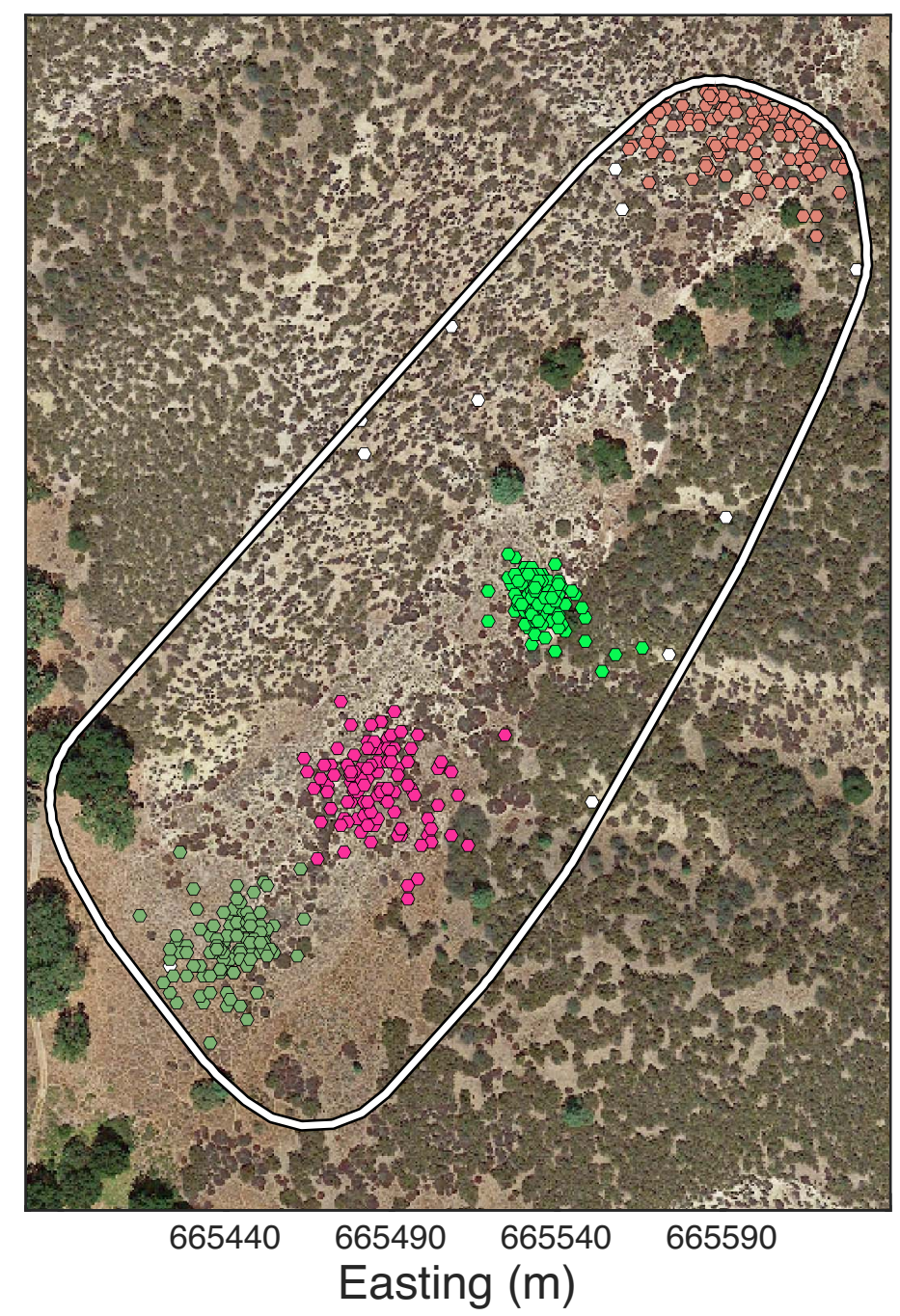




\section{LT Session S10 Home Range Center Estimates EP}
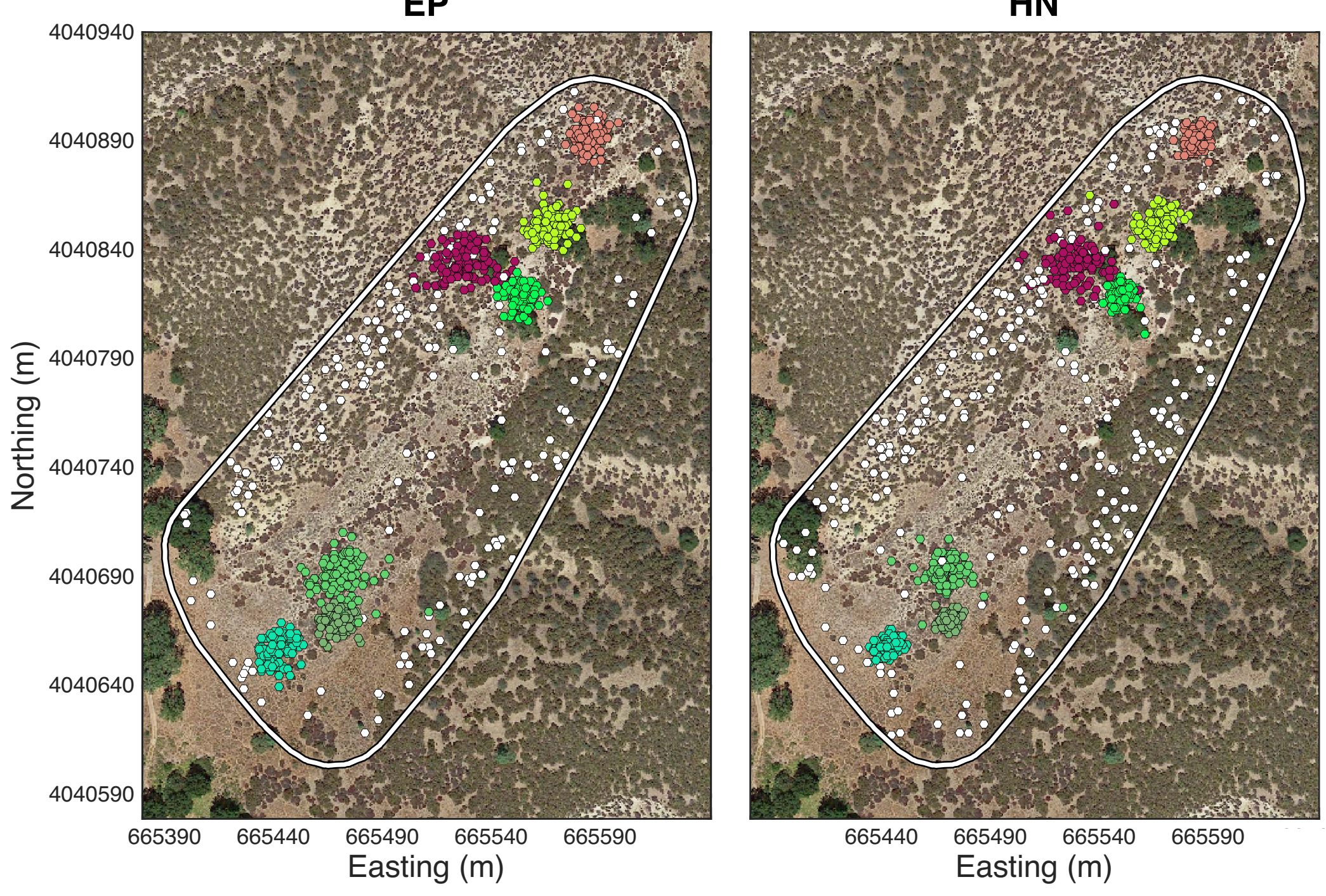


\section{CT-LT Session S1 Home Range Center Estimates}

EP

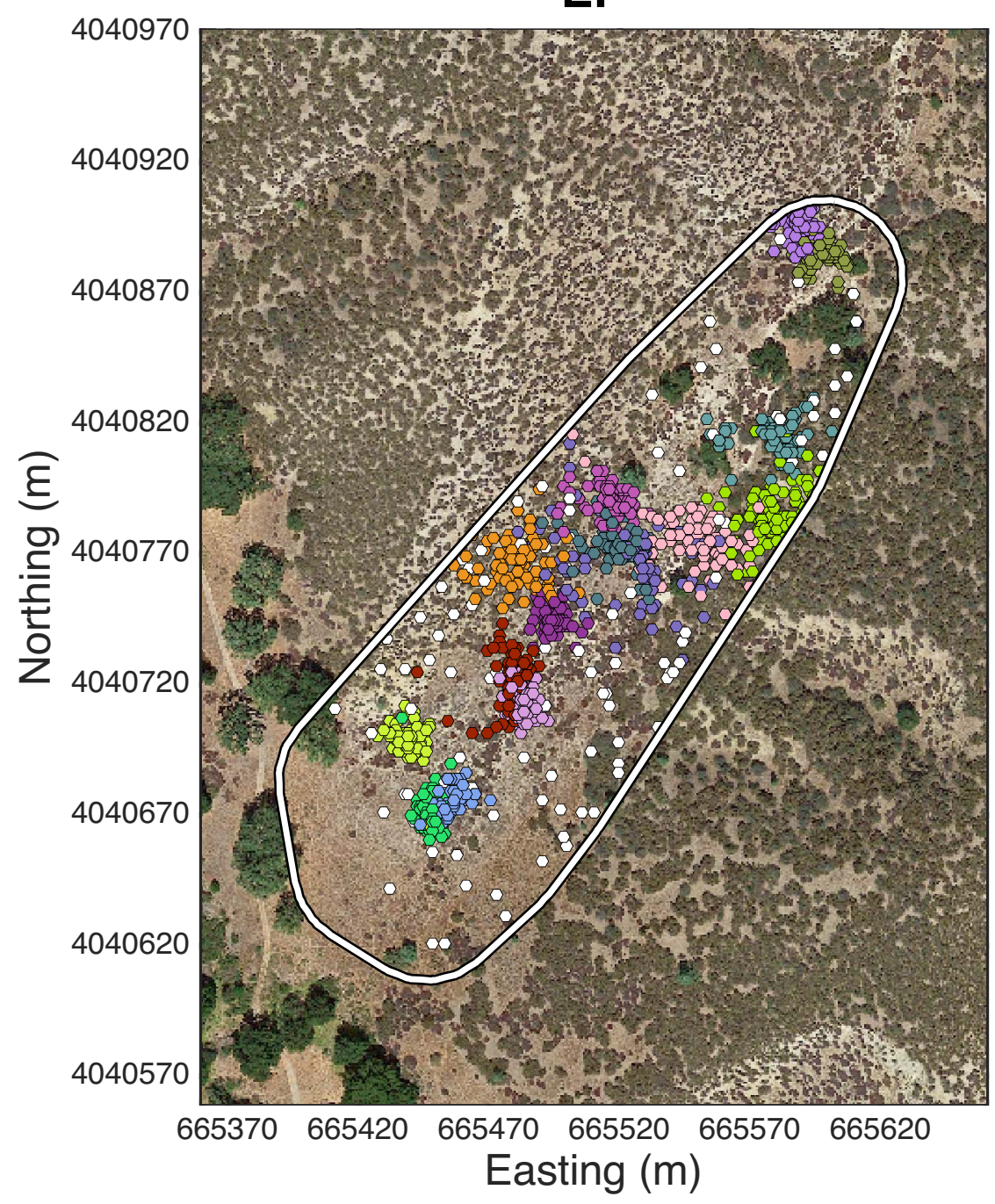

HN

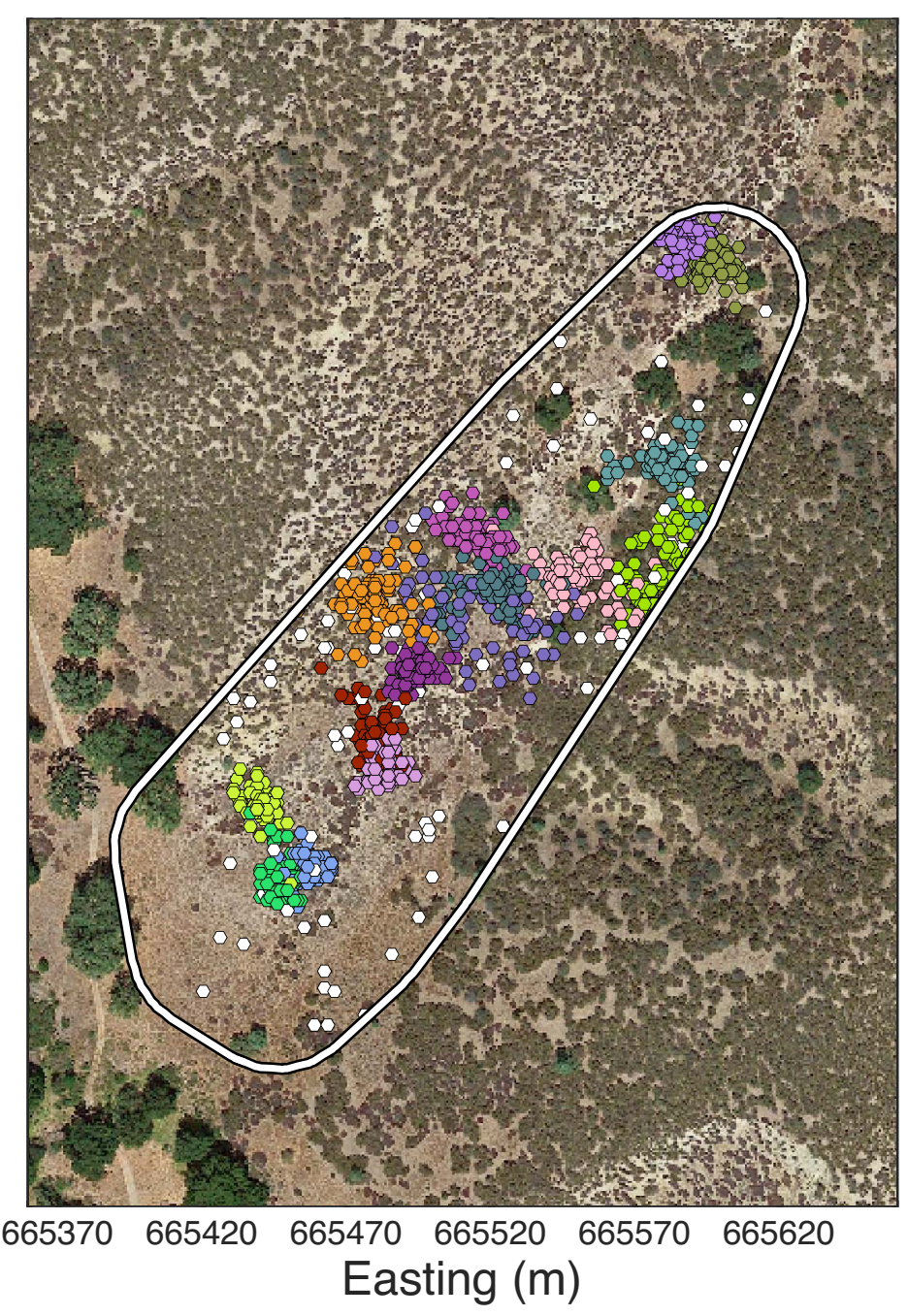




\section{CT-LT Session S2 Home Range Center Estimates}
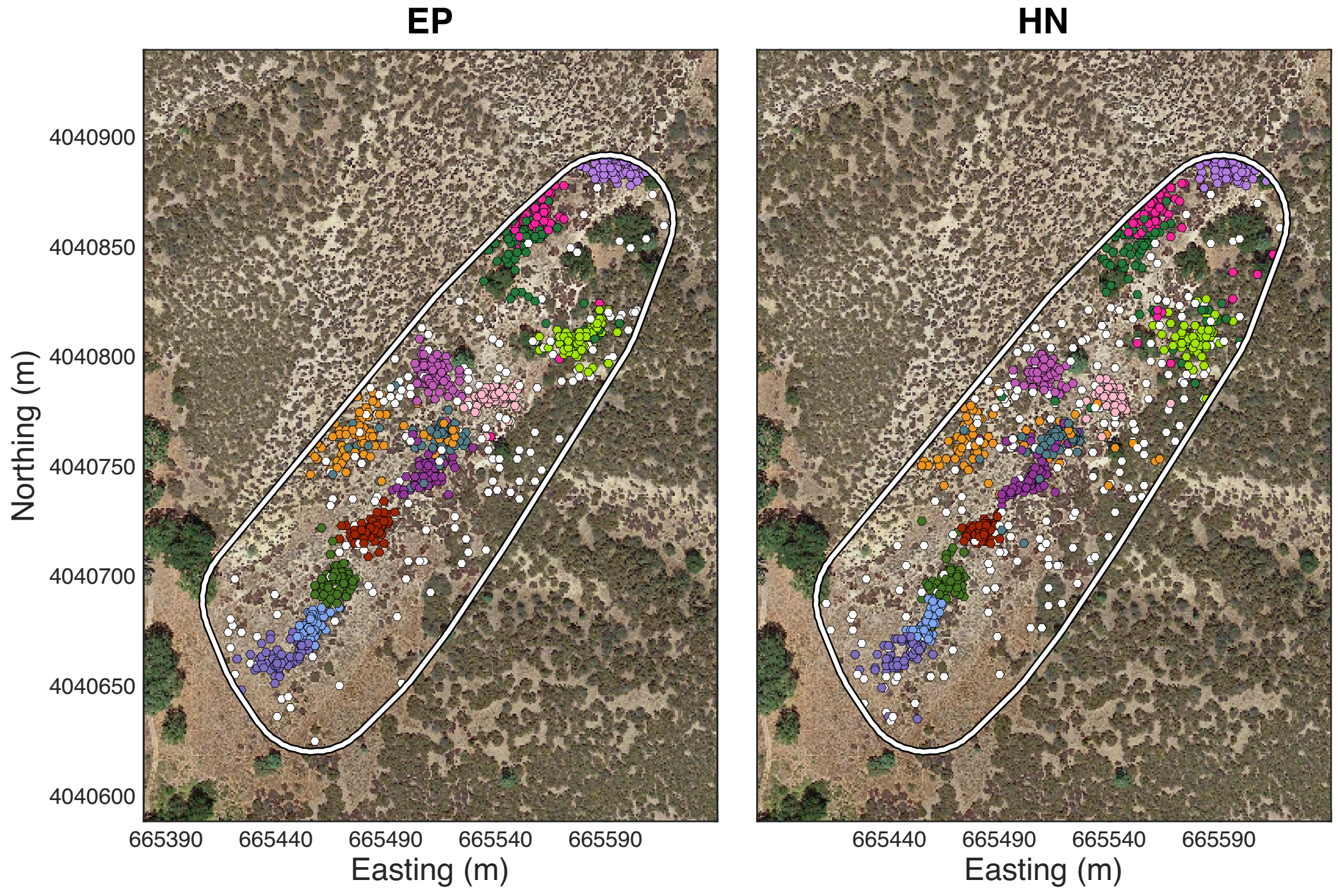


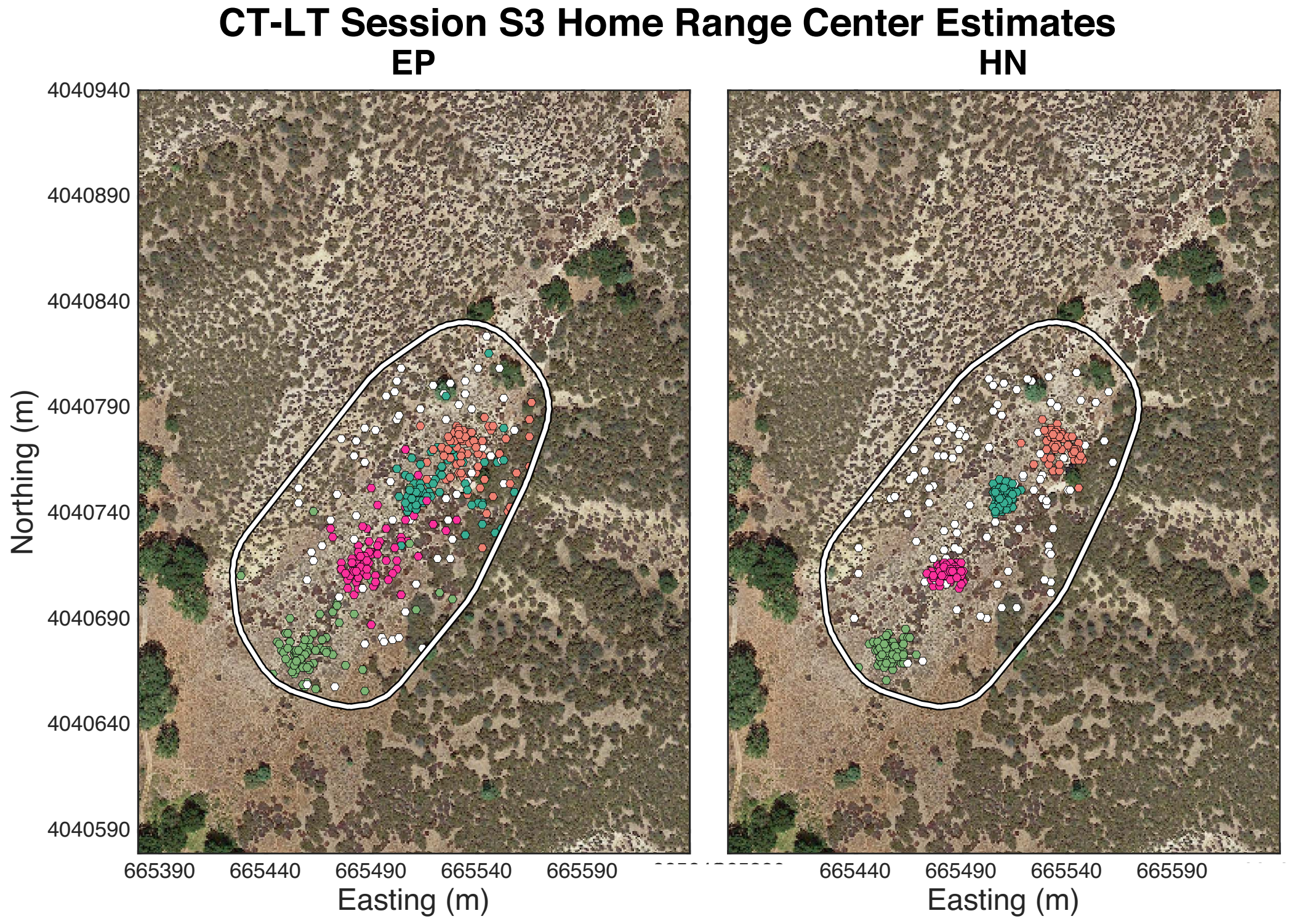


CT-LT Session S4 Home Range Center Estimates EP

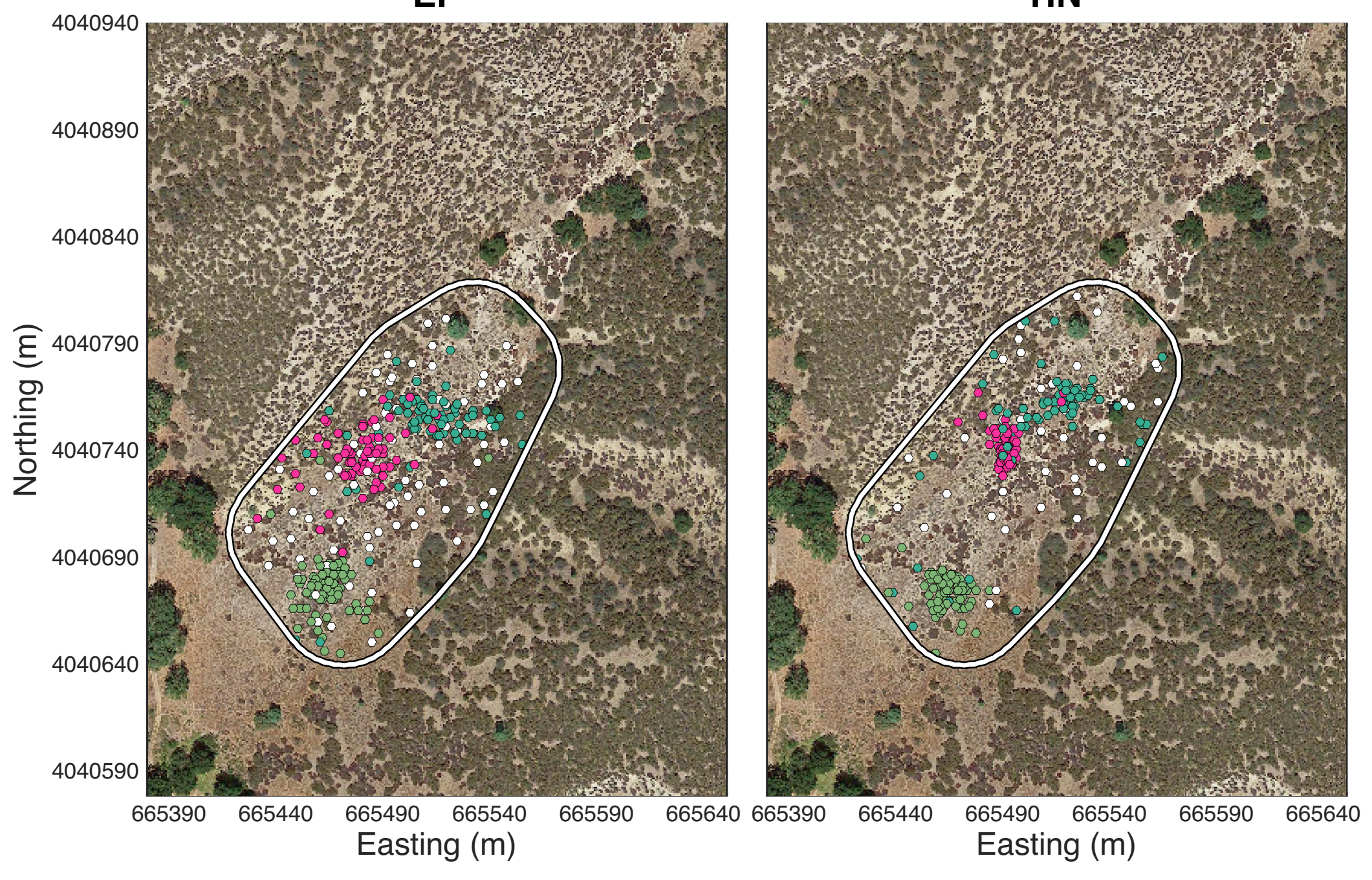




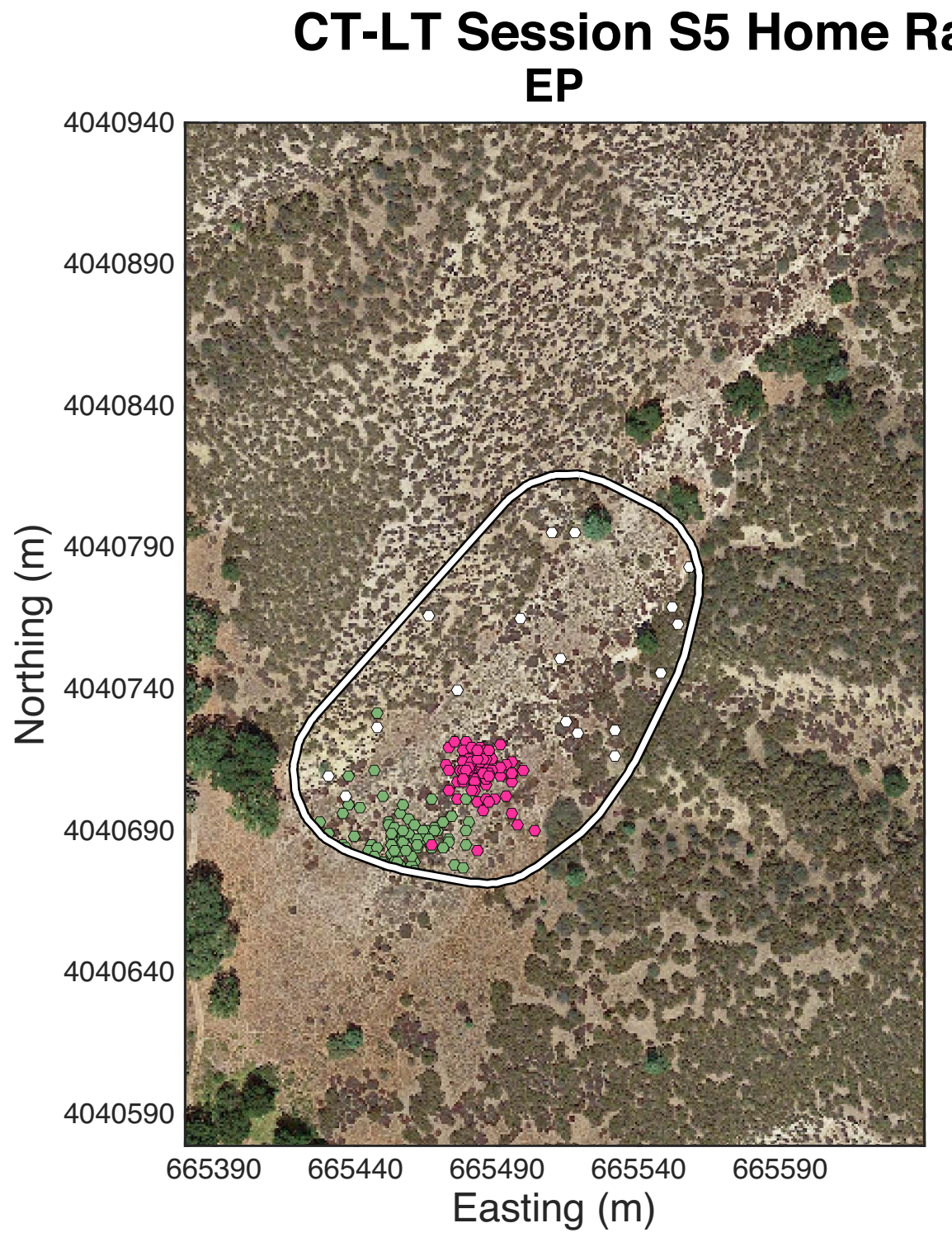

\section{Range Center Estimates \\ HN}

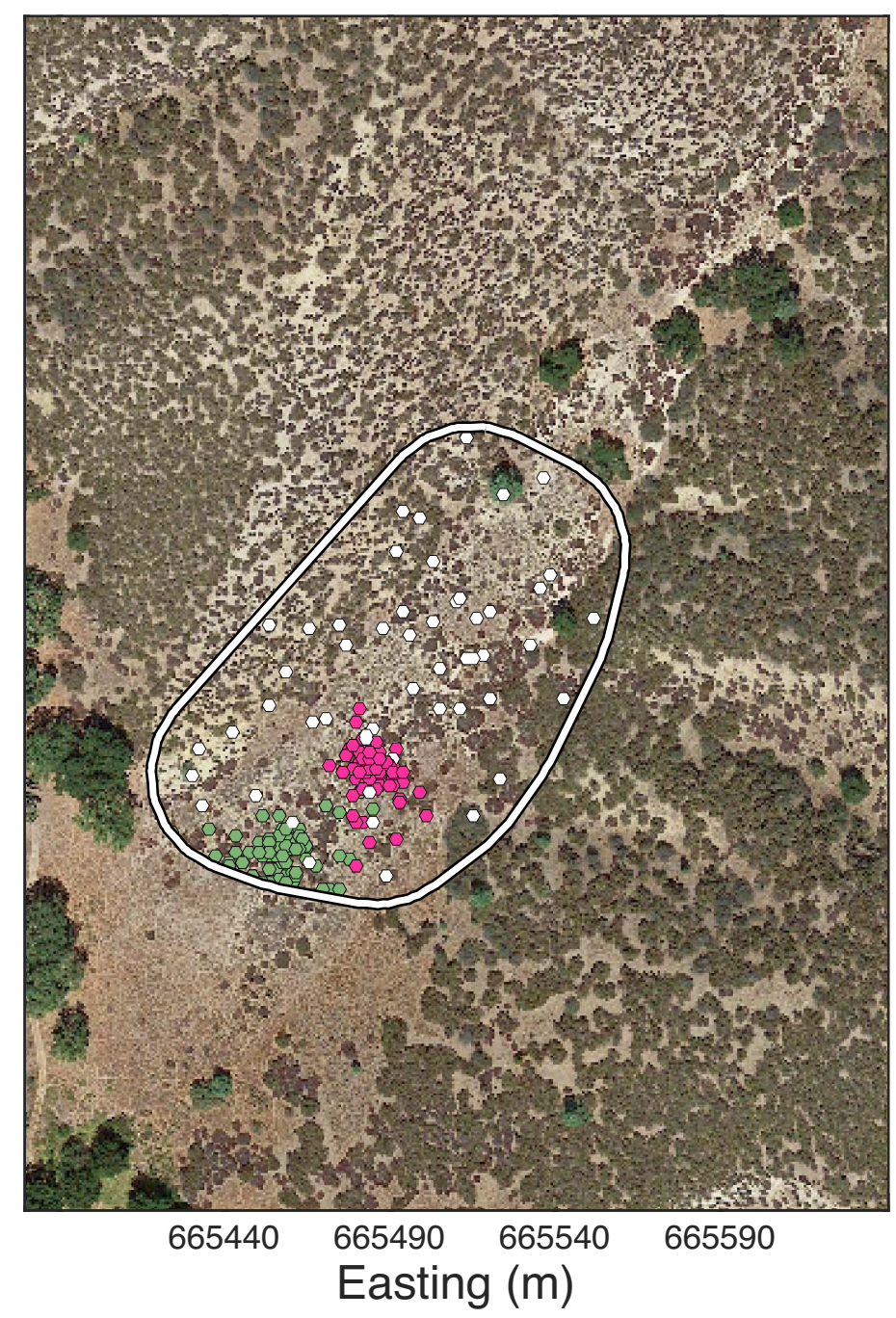




\section{CT-LT Session S6 Home Range Center Estimates \\ EP \\ HN}

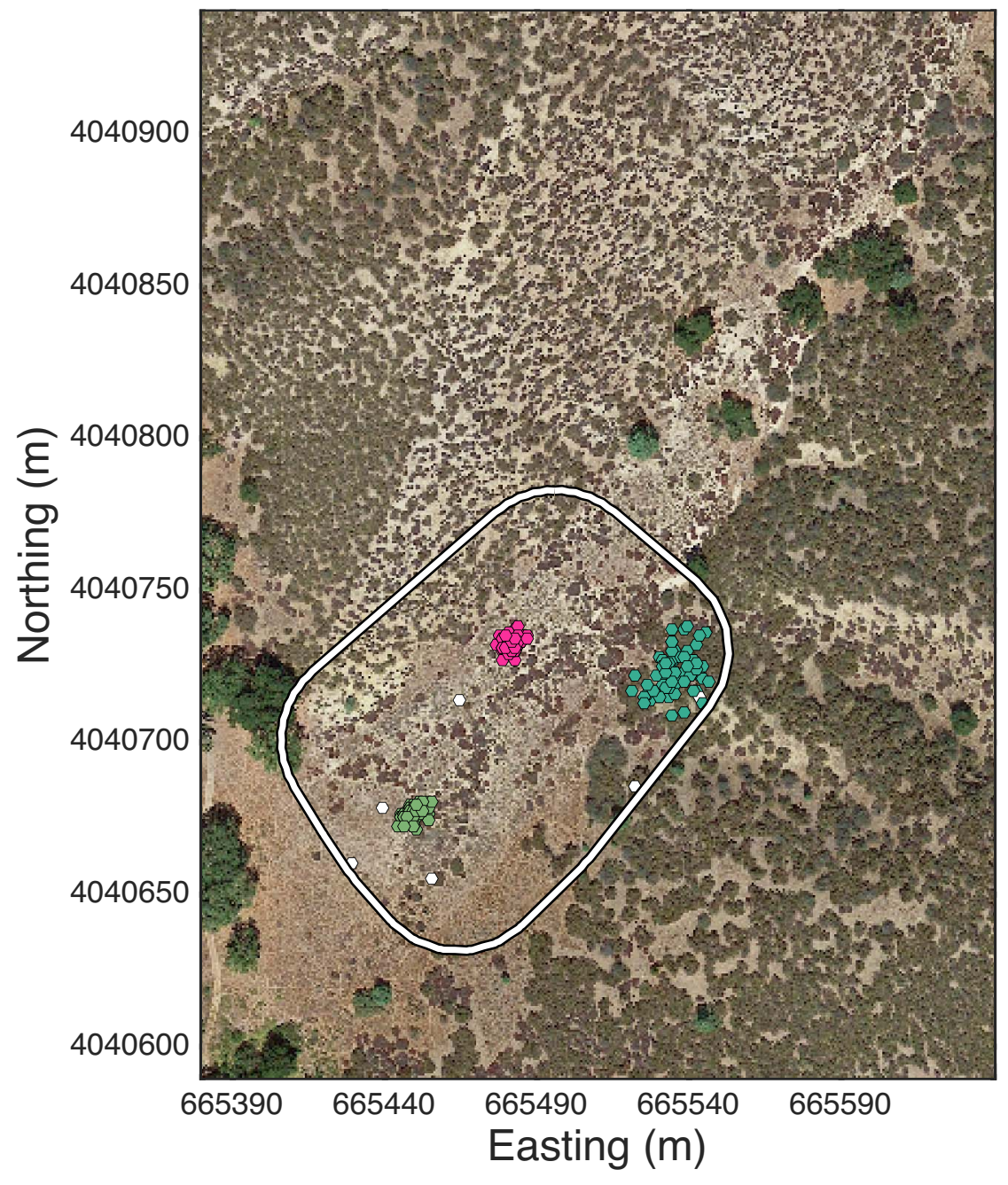

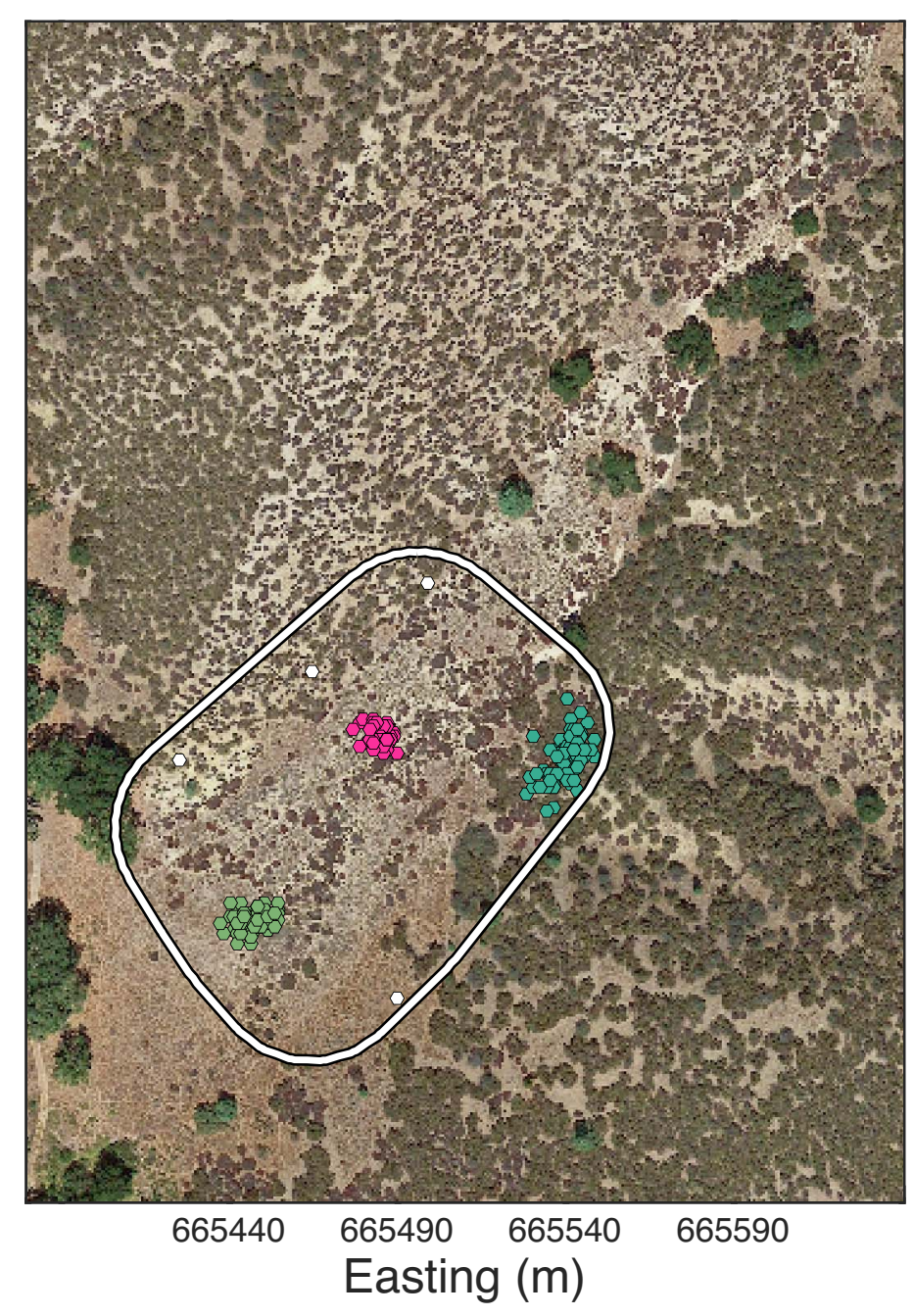




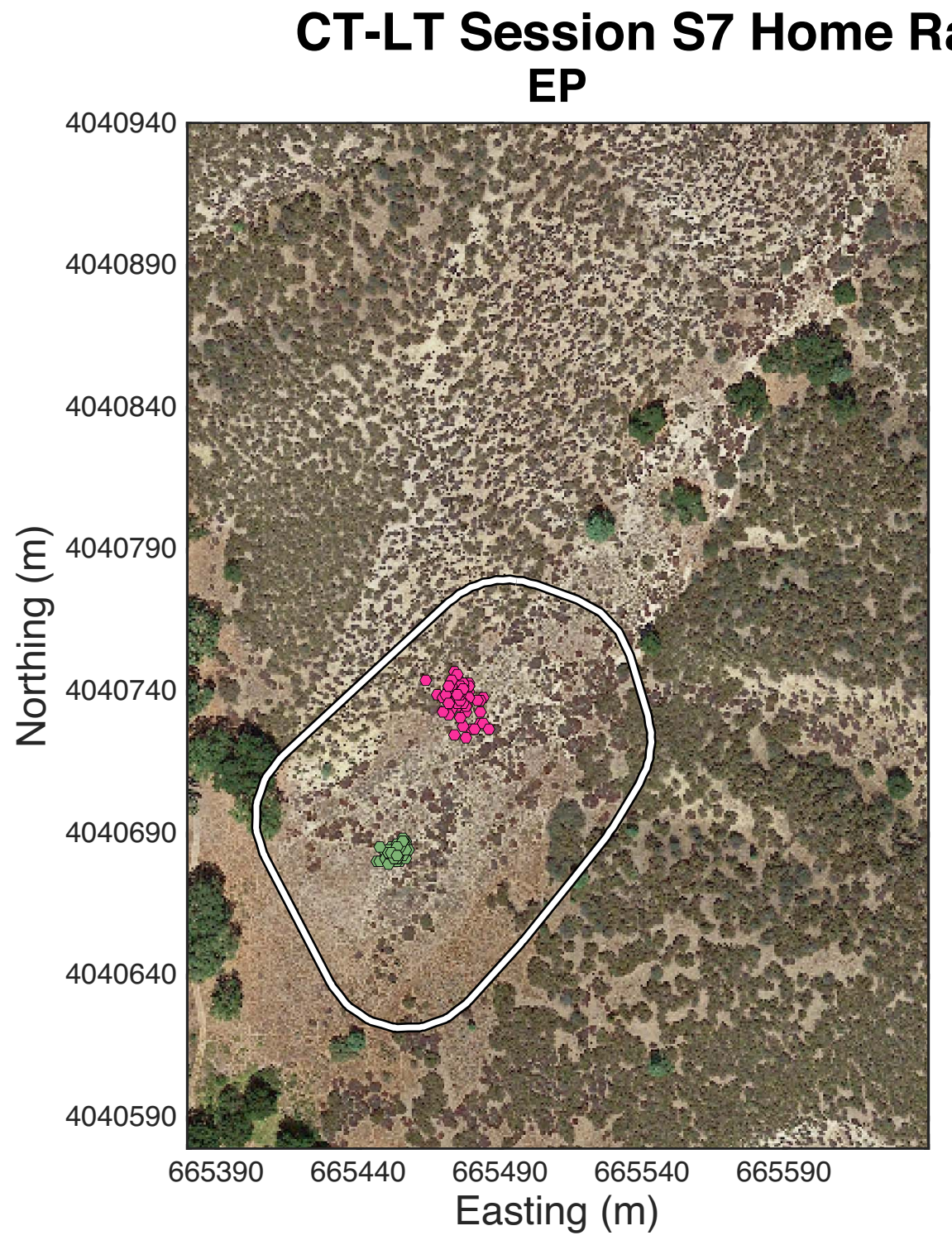

\section{Range Center Estimates \\ HN}

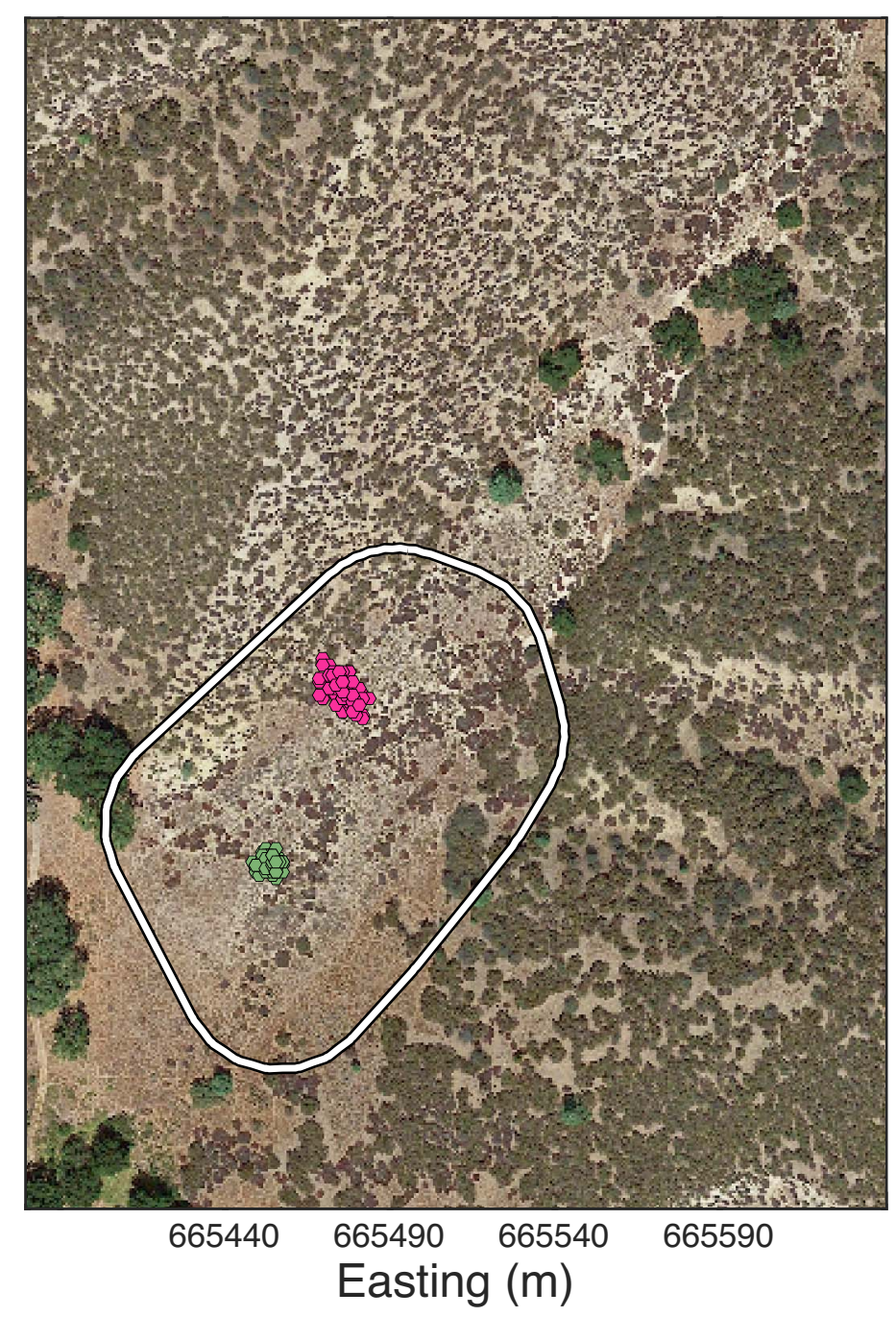


CT-LT Session S8 Home Range Center Estimates

EP

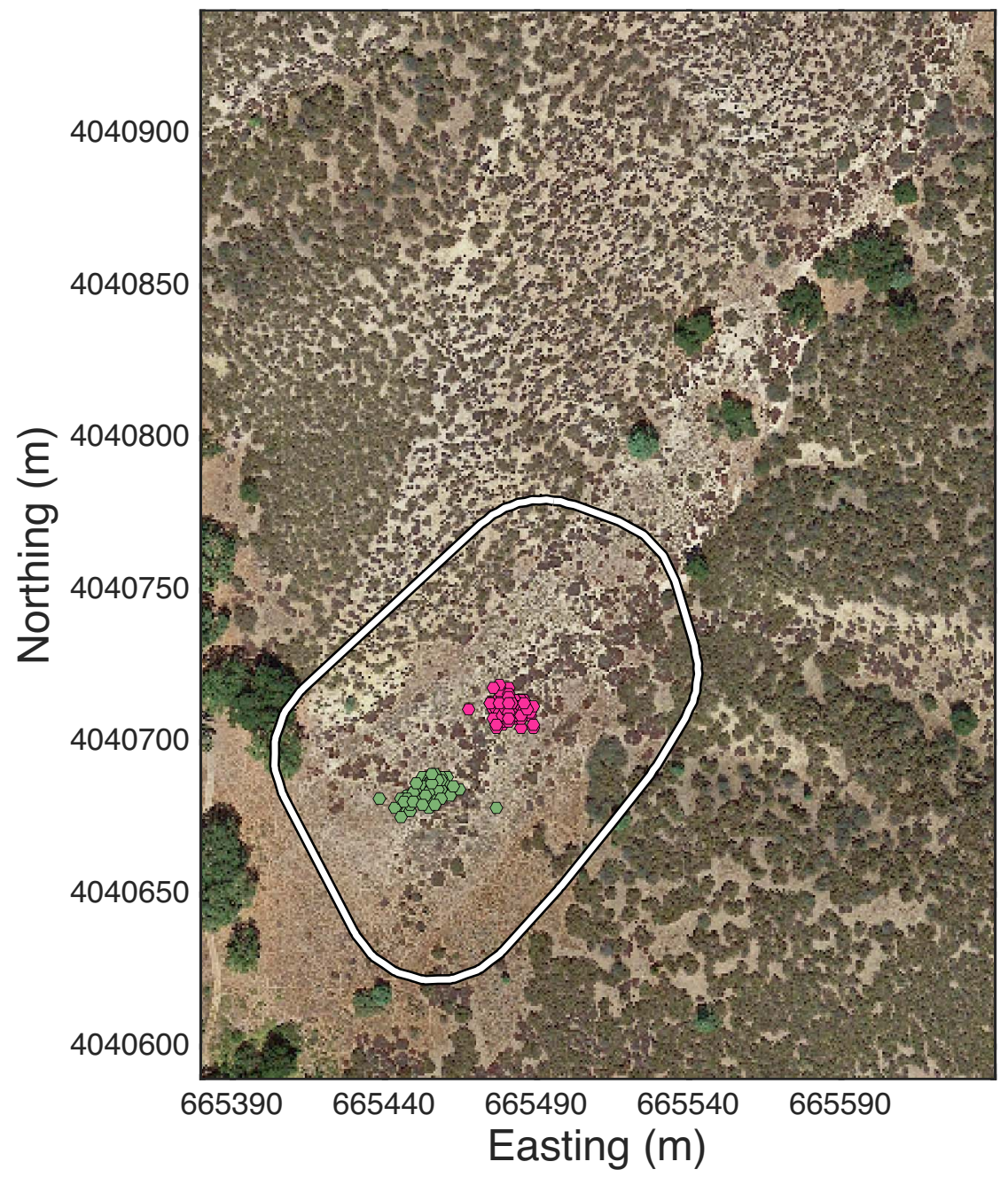

HN

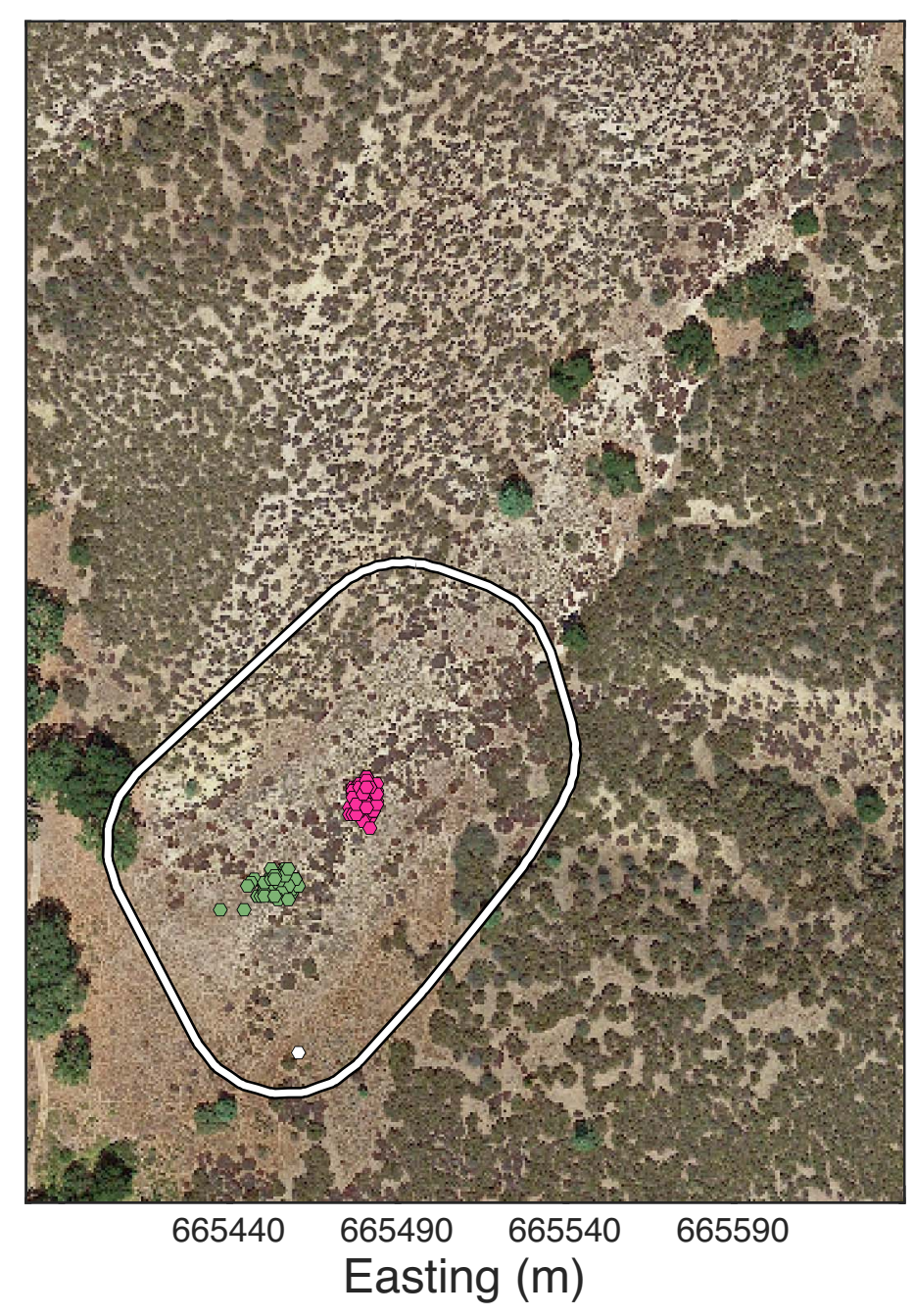




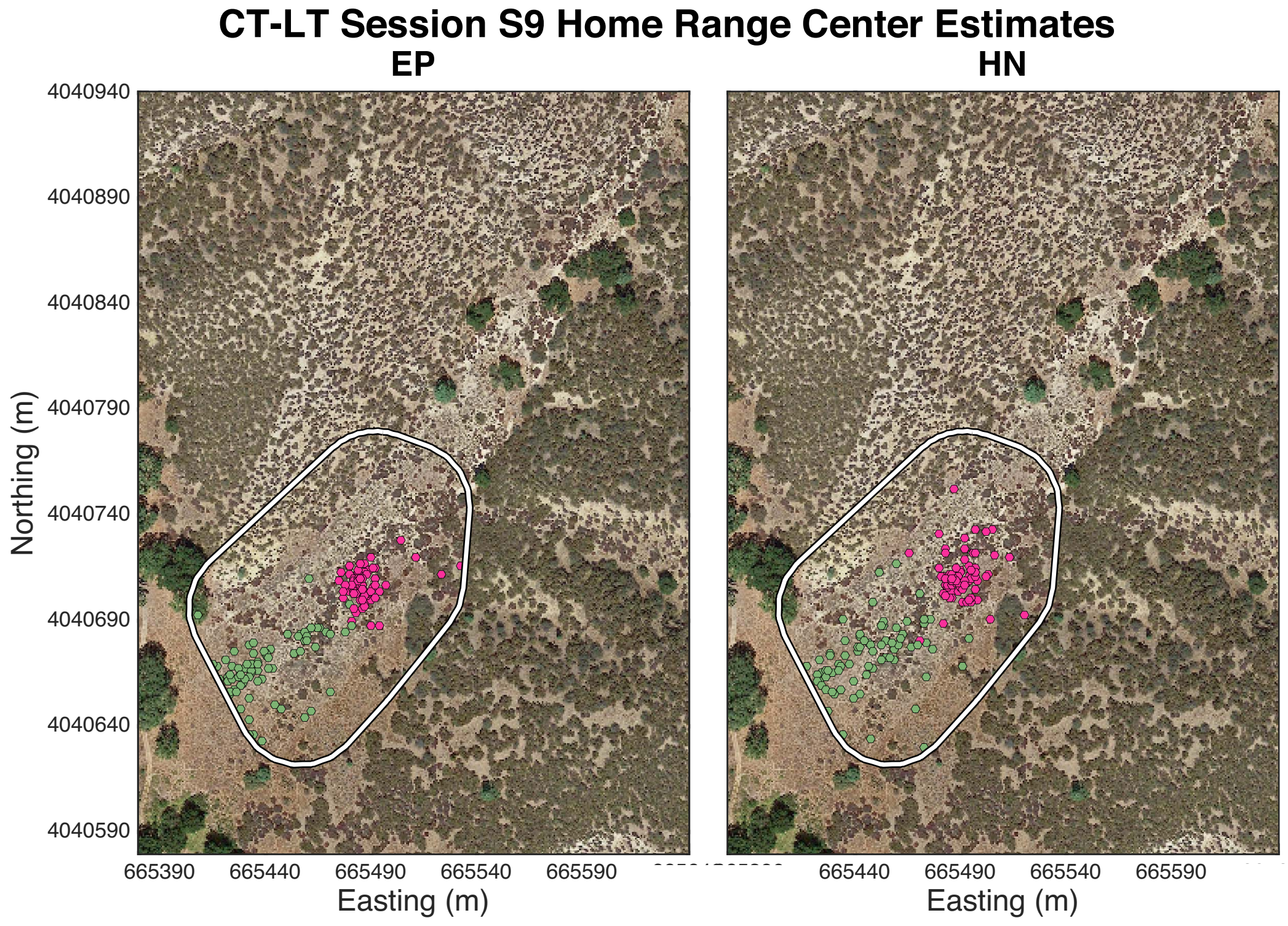




\section{CT-LT Session S10 Home Range Center Estimates}

EP
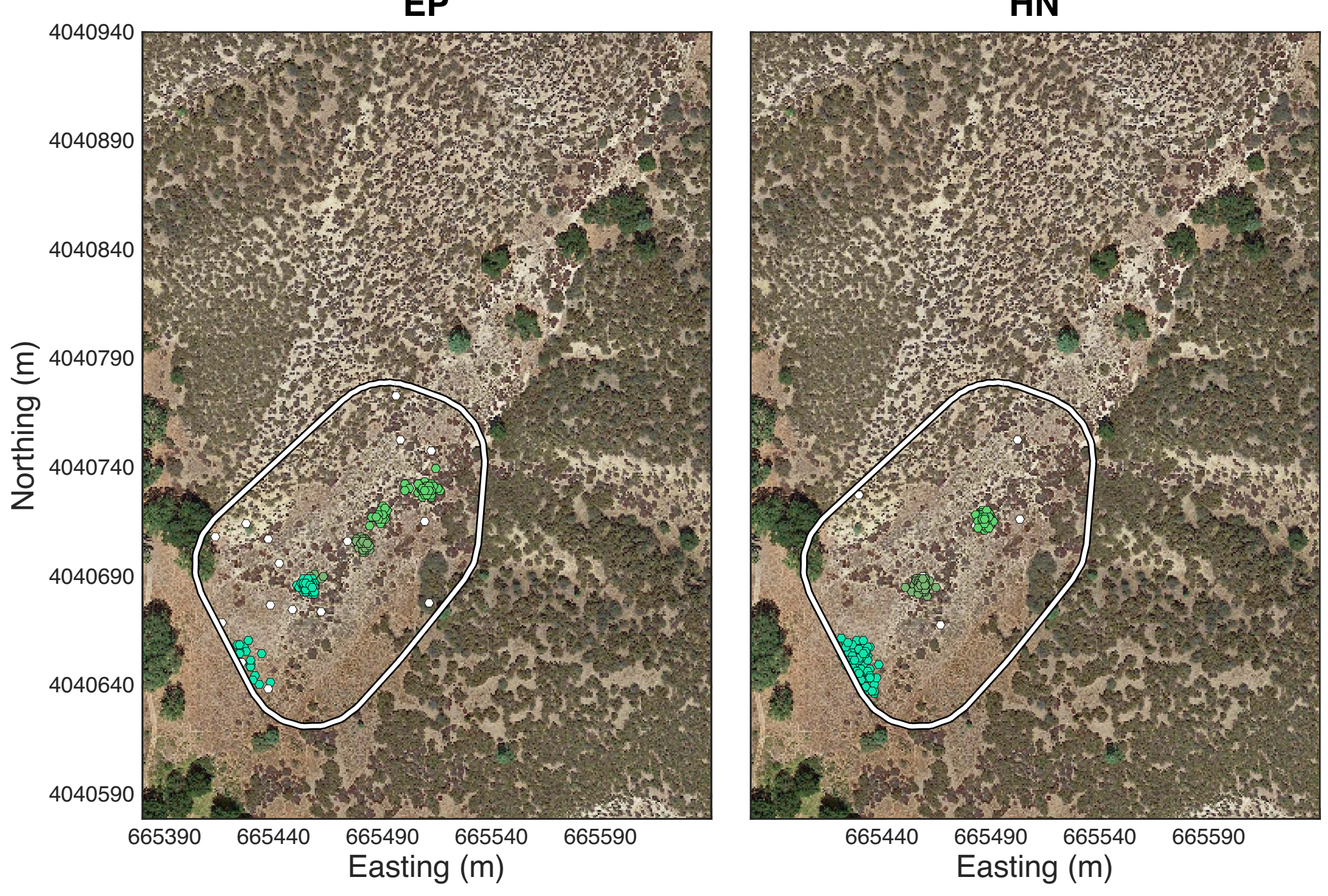


\section{CTMx-LT Session S1+S2 Home Range Center Estimates}
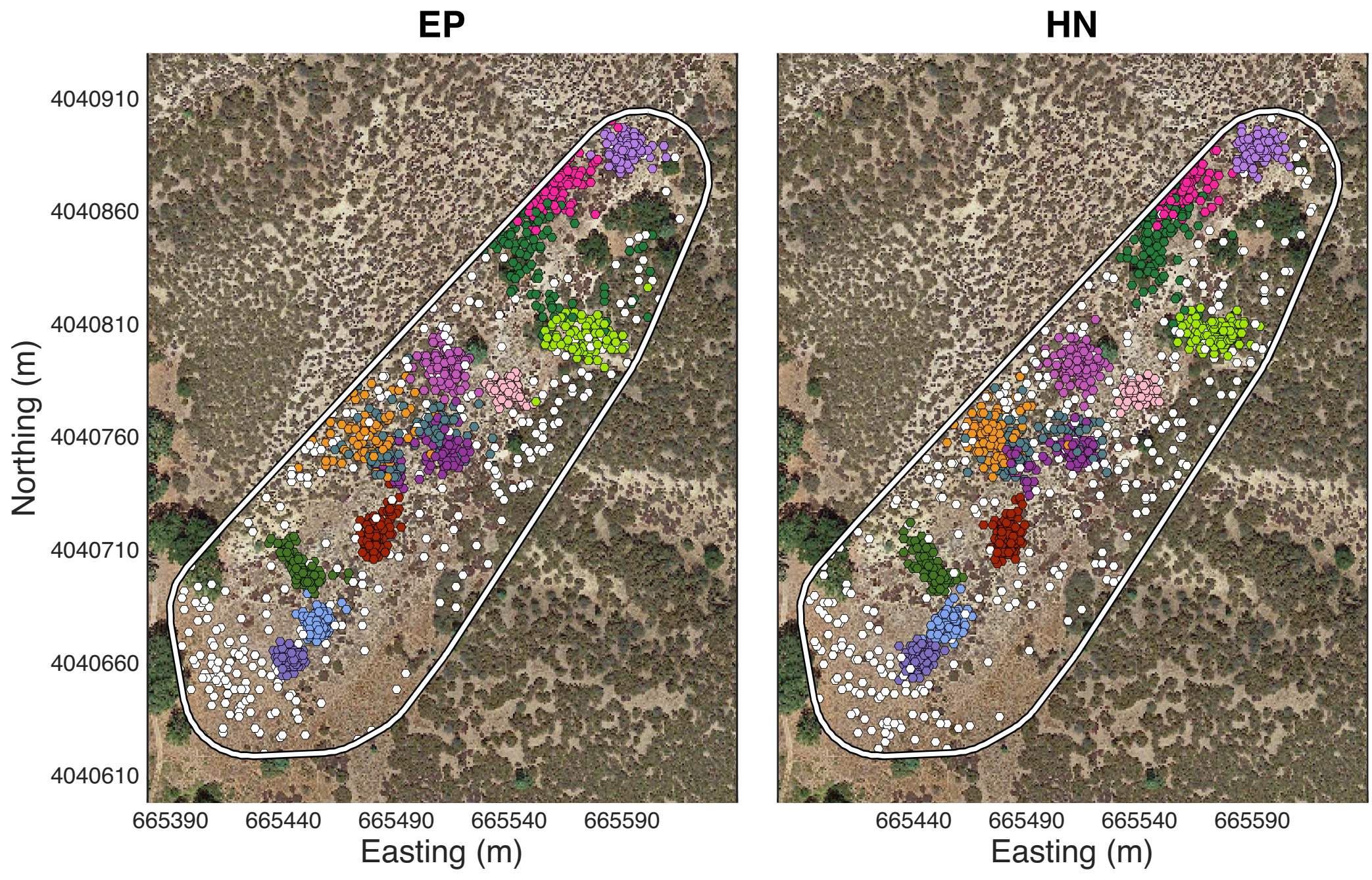


\section{CTMx-LT Session S3+S4 Home Range Center Estimates}
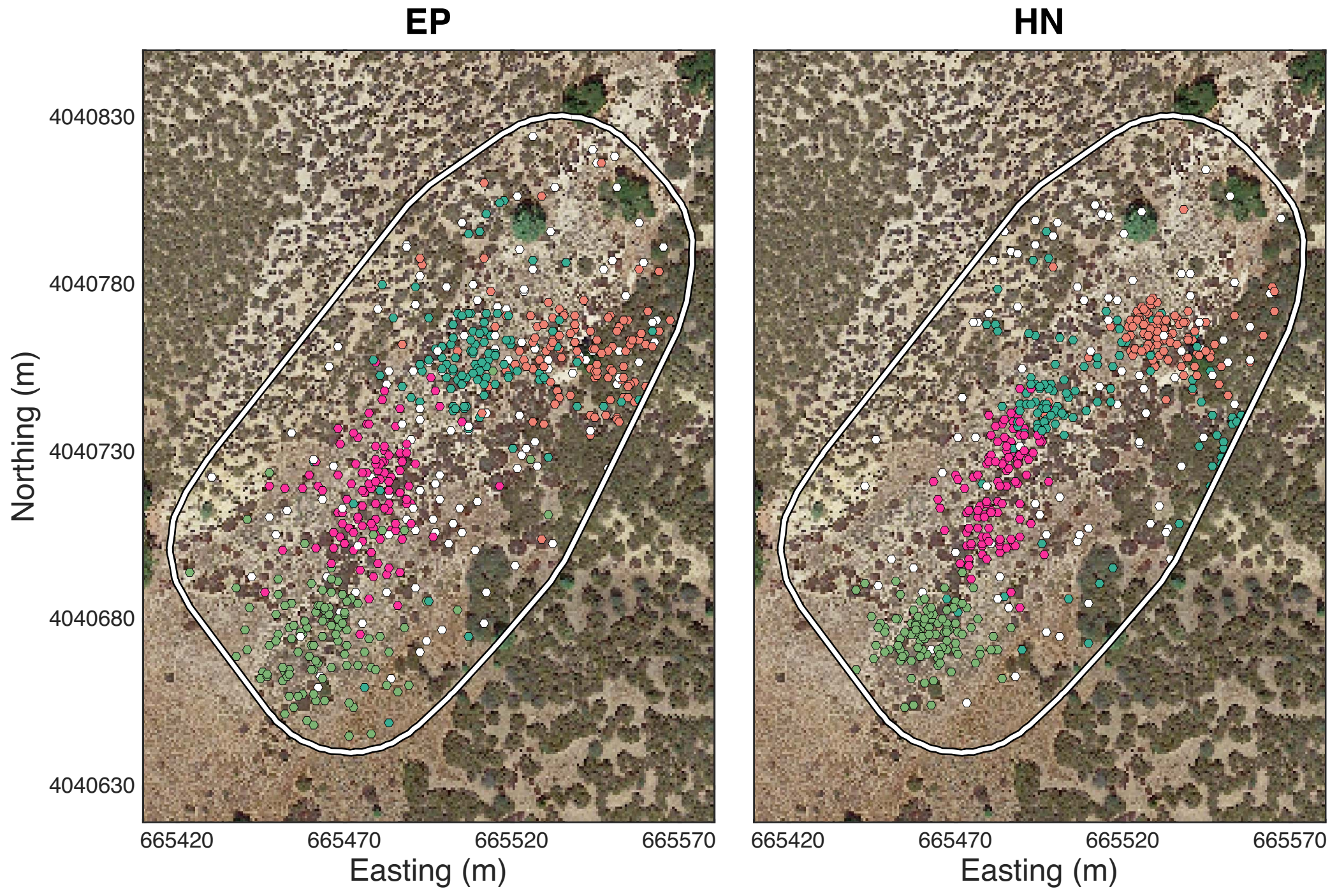


\section{CTMx-LT Session S4+S5 Home Range Center Estimates}

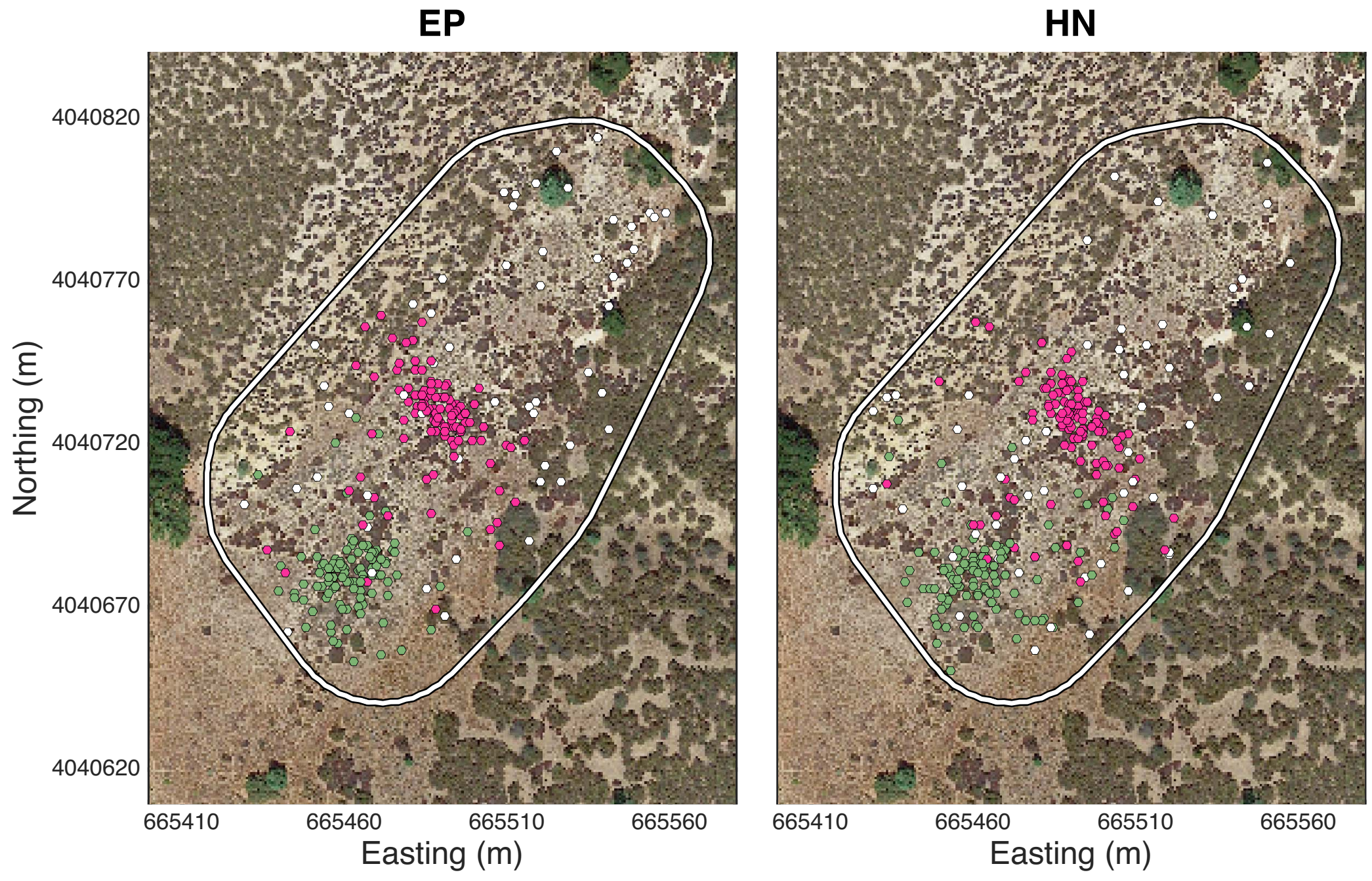




\section{CTMx-LT Session S5+S6 Home Range Center Estimates}
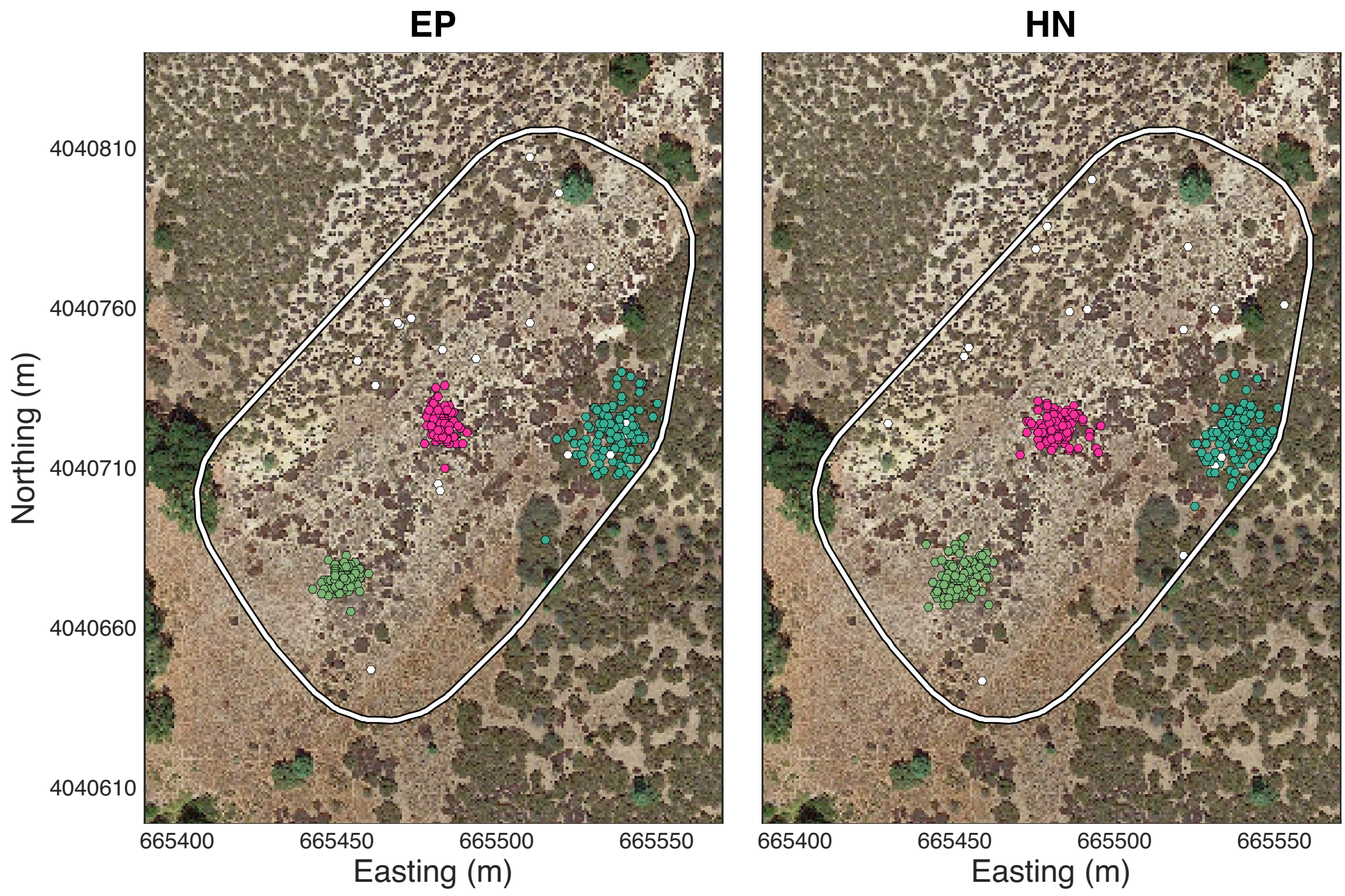


\section{CTMx-LT Session S6+S7 Home Range Center Estimates}
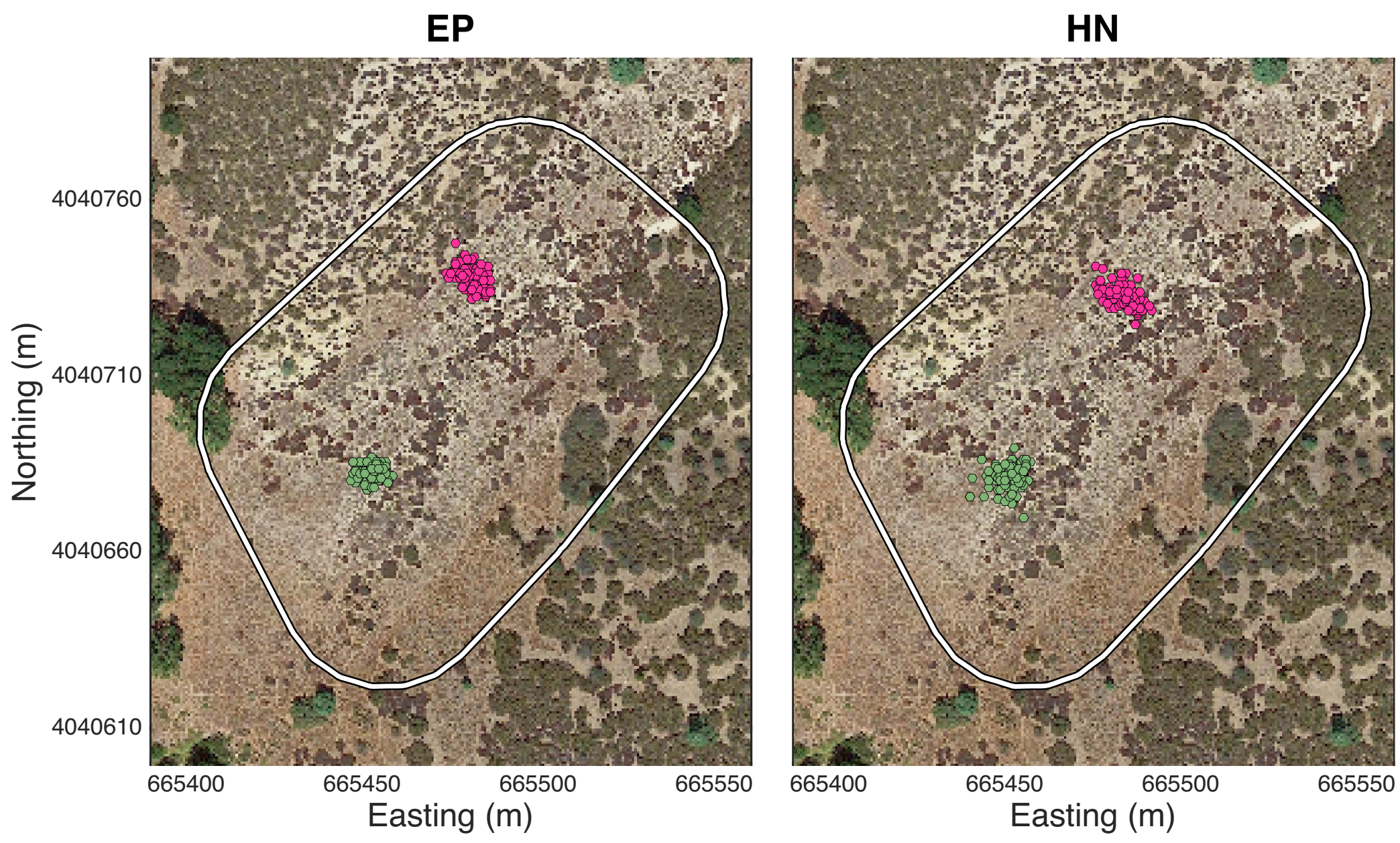


\section{Appendix E. Home Range Center Density Plots}

This appendix details the home range center estimates for two datasets: CT and CTMix. For every session of data, a density plot of home range center $\left(\boldsymbol{s}_{i}\right)$ estimates is displayed. Unlike the home range center estimates from the live trapping data, no individual identity is known for the camera trapping data. So a density plot is used that shows the areas of greatest home range center concentrations. Blue areas are those with few home range center estimates, and red areas are those with high densities of home range center estimates. Areas with small, distinct patches of red and orange have better convergence of home range centers. Each density plot is standardized to a width of 120 pixels. On each page, a session's home range center density plots are shown for all four implementations. The top two show the results for each detection function without a prior on $\sigma$ (the EP and $\mathrm{HN}$ implementations). The bottom two show the density plot for each detection function with a prior on $\sigma$ (the EP-SP and HN-SP implementations). 
CT Session S1 Home Range Center Density Plots

EP

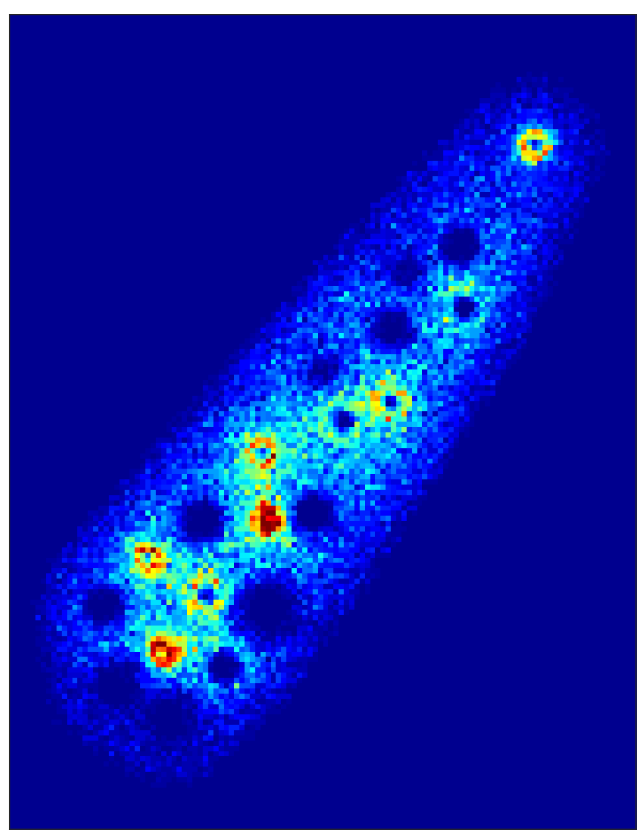

EP-SP

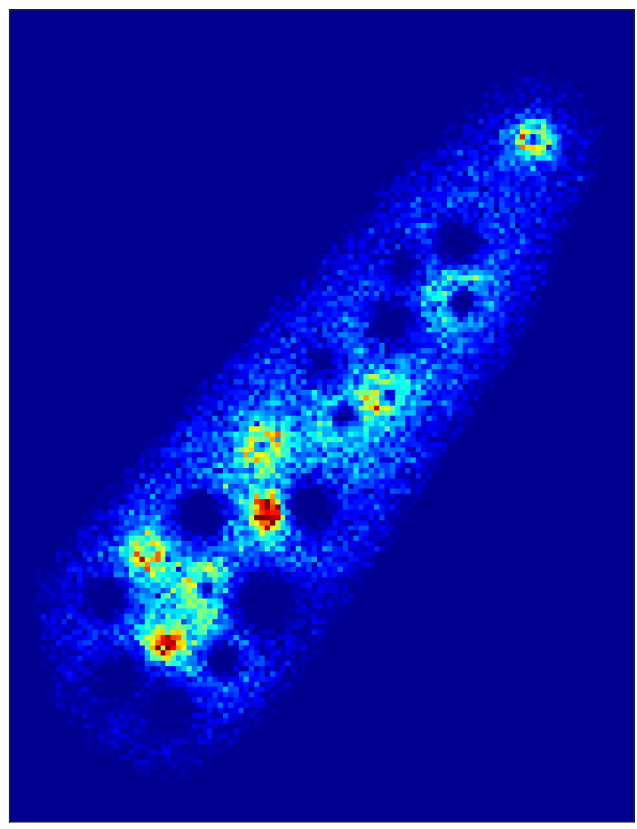

HN

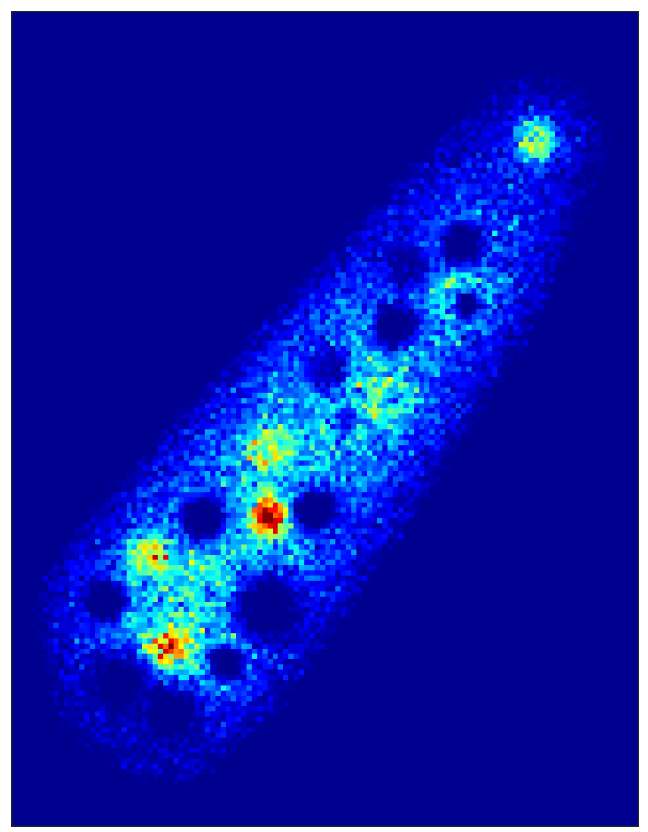

HN-SP

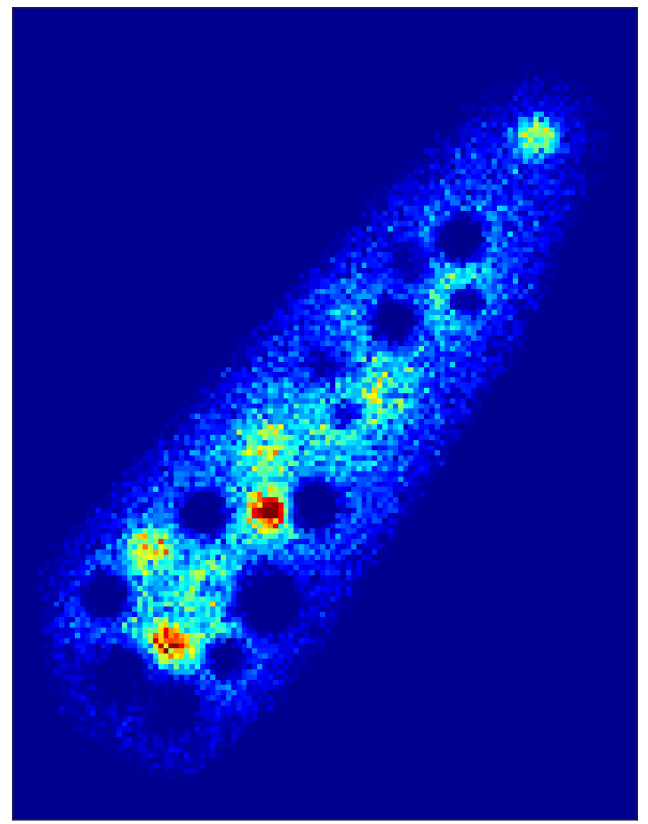


CT Session S2 Home Range Center Density Plots

EP

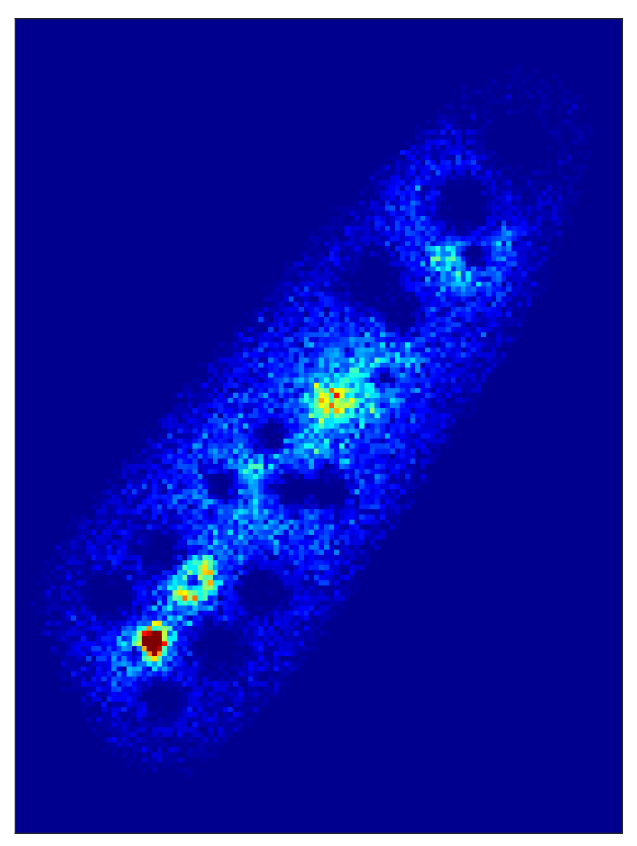

EP-SP

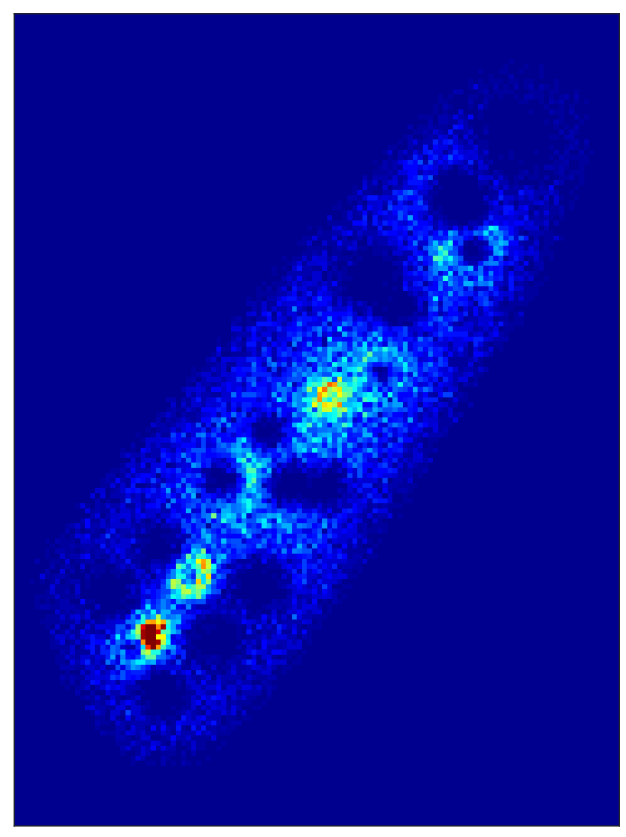

HN

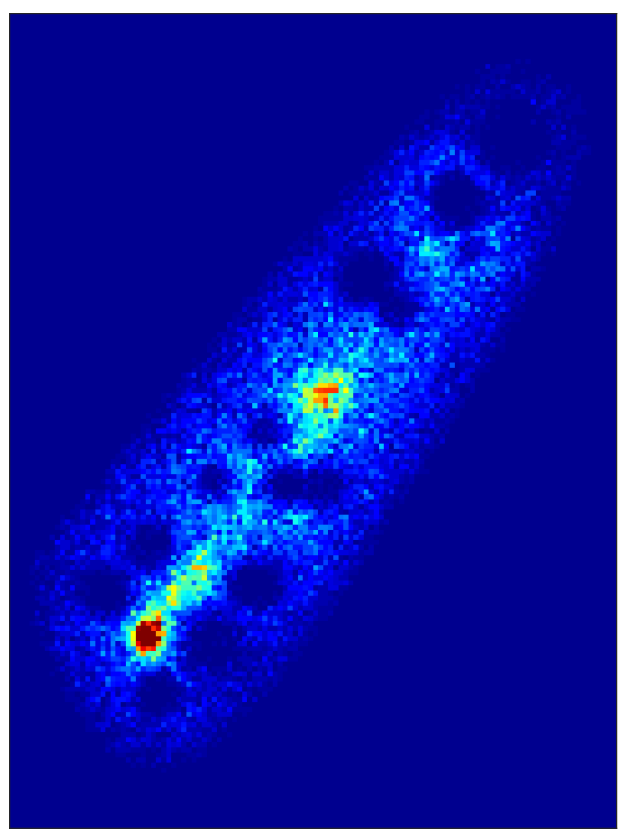

HN-SP

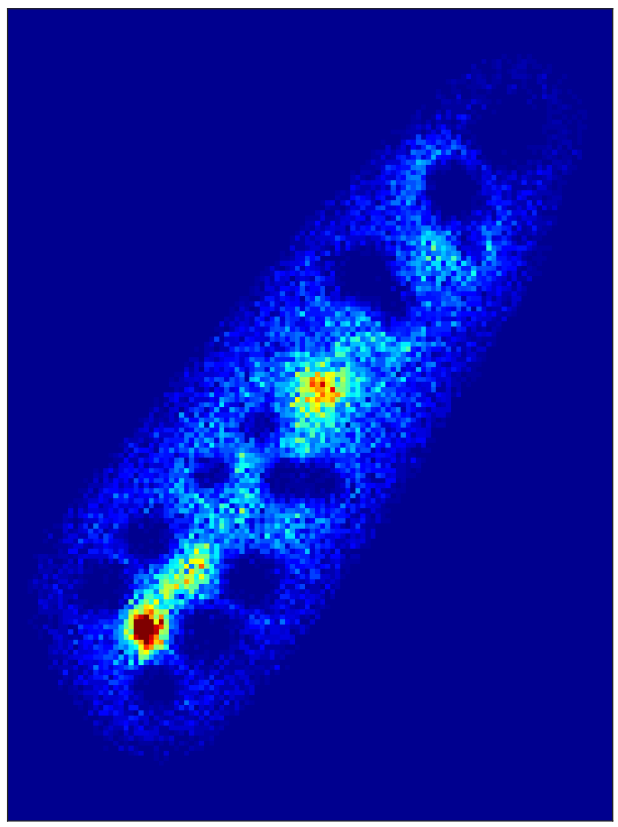


CT Session S3 Home Range Center Density Plots

EP

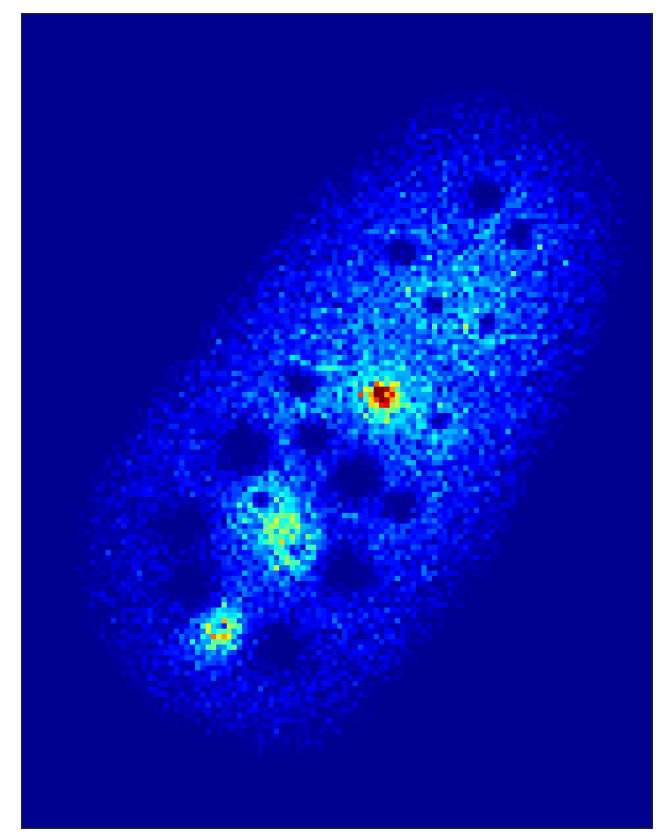

EP-SP

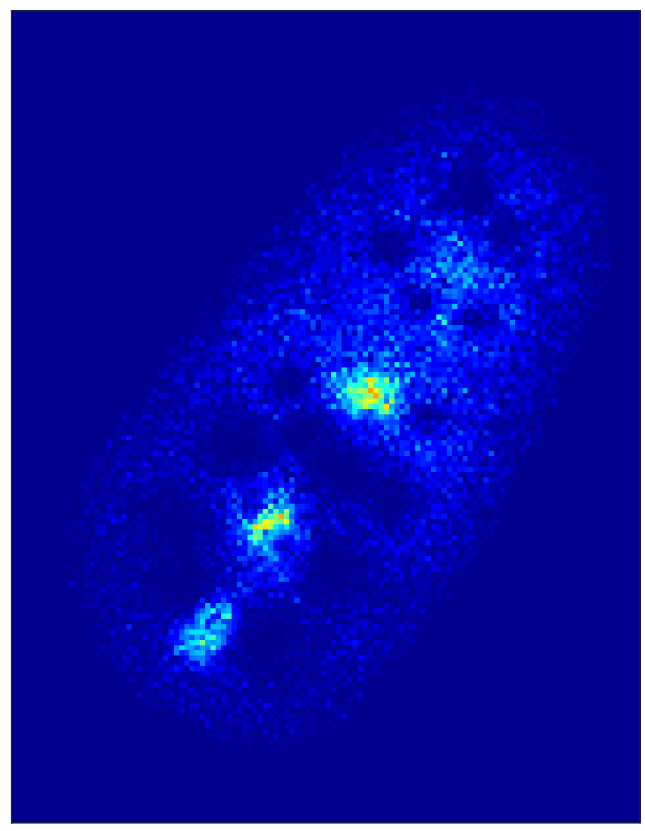

HN

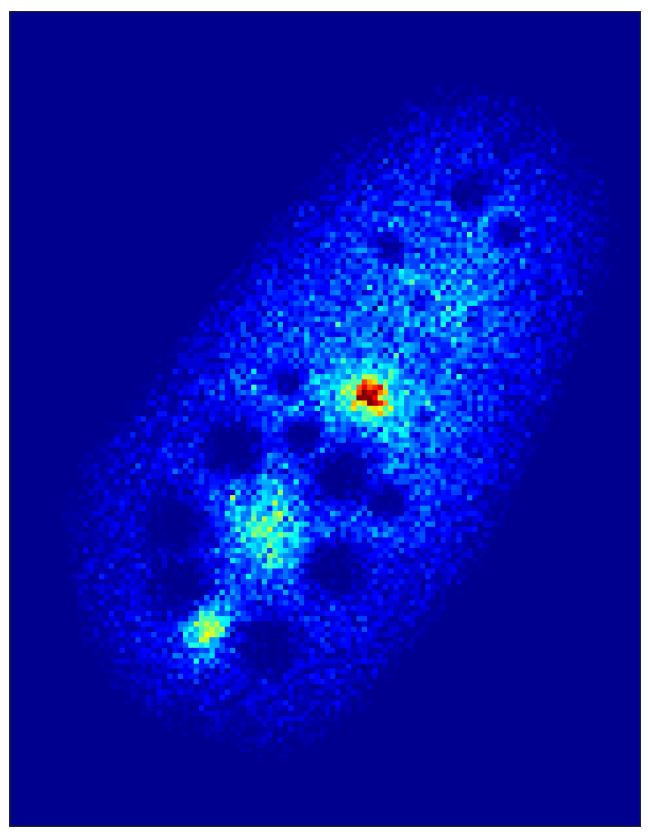

HN-SP

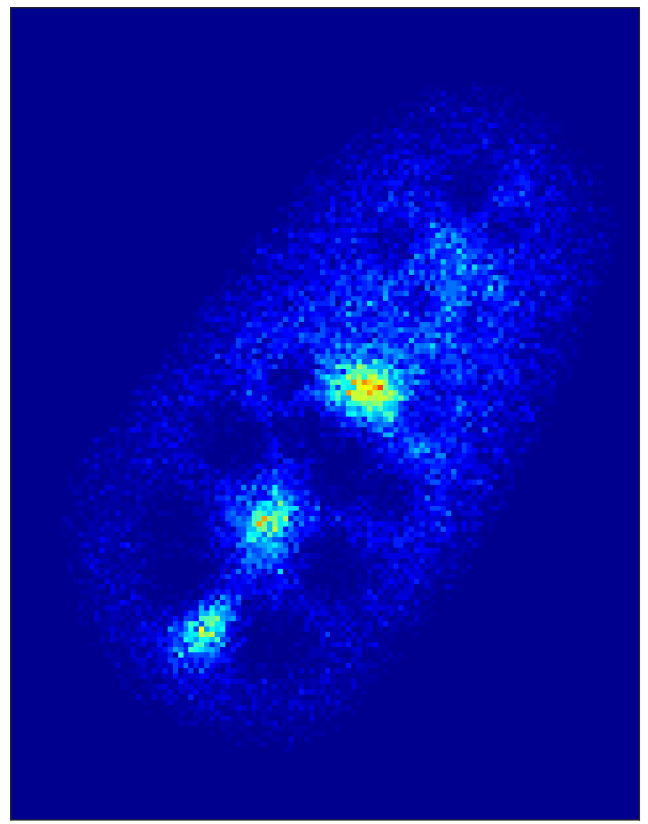


CT Session S4 Home Range Center Density Plots

EP

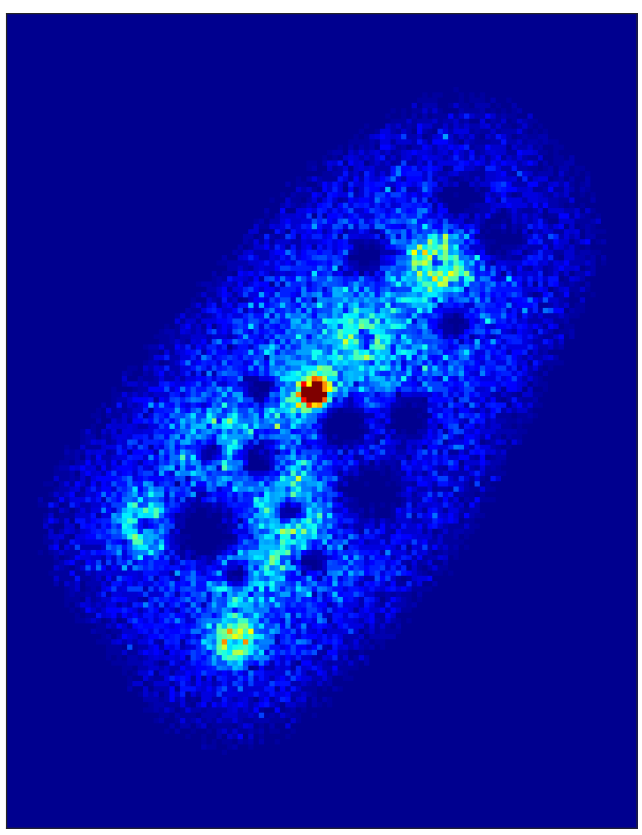

EP-SP

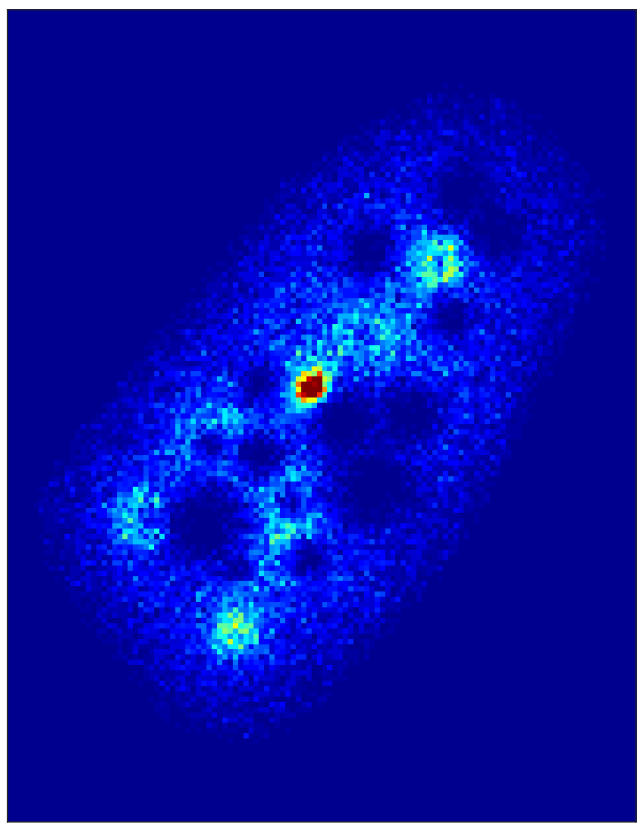

HN

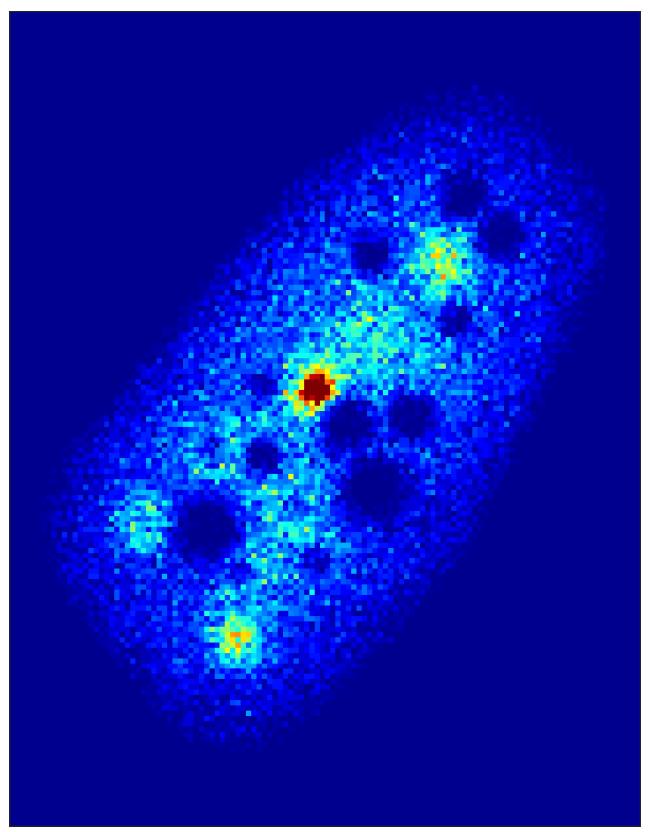

HN-SP

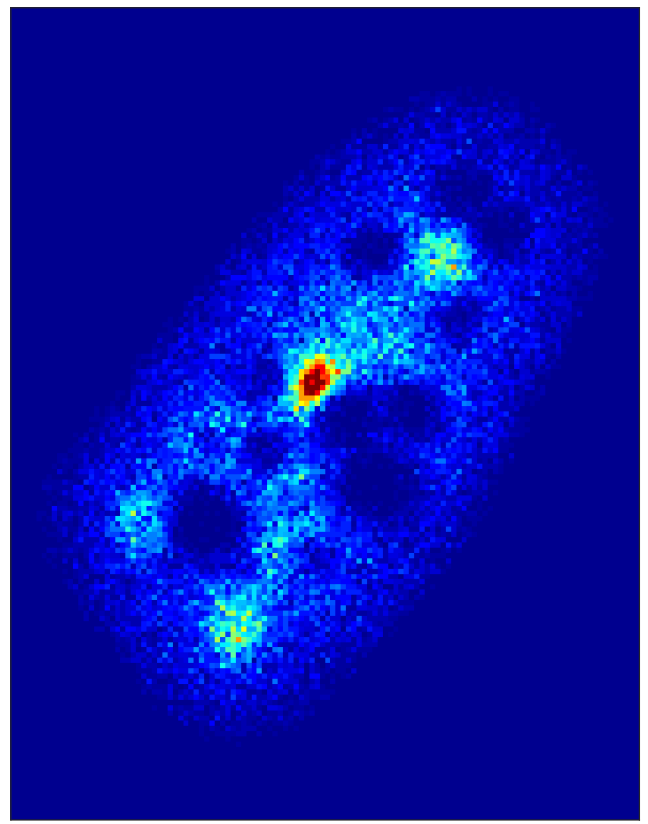




\section{CT Session S5 Home Range Center Density Plots}

EP

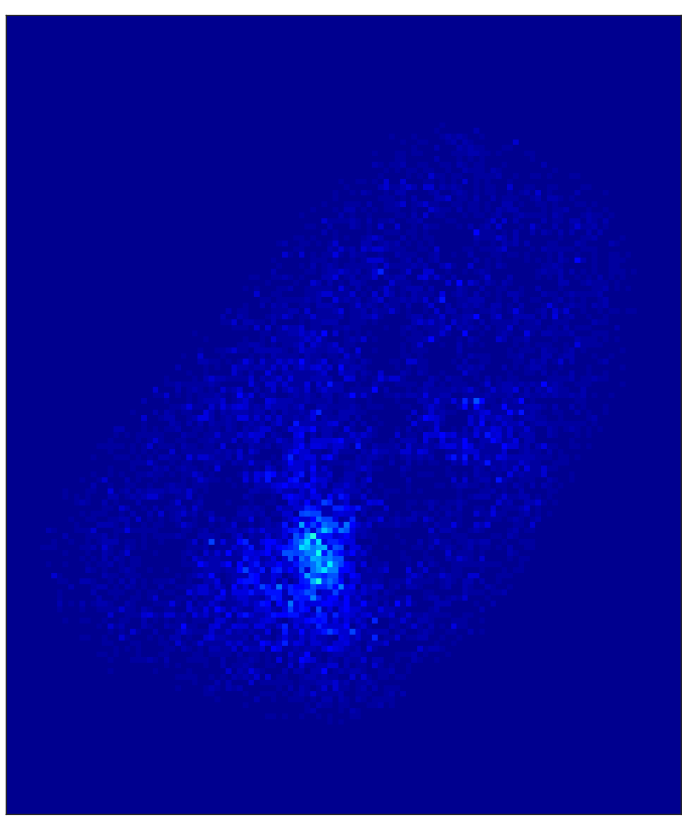

EP-SP

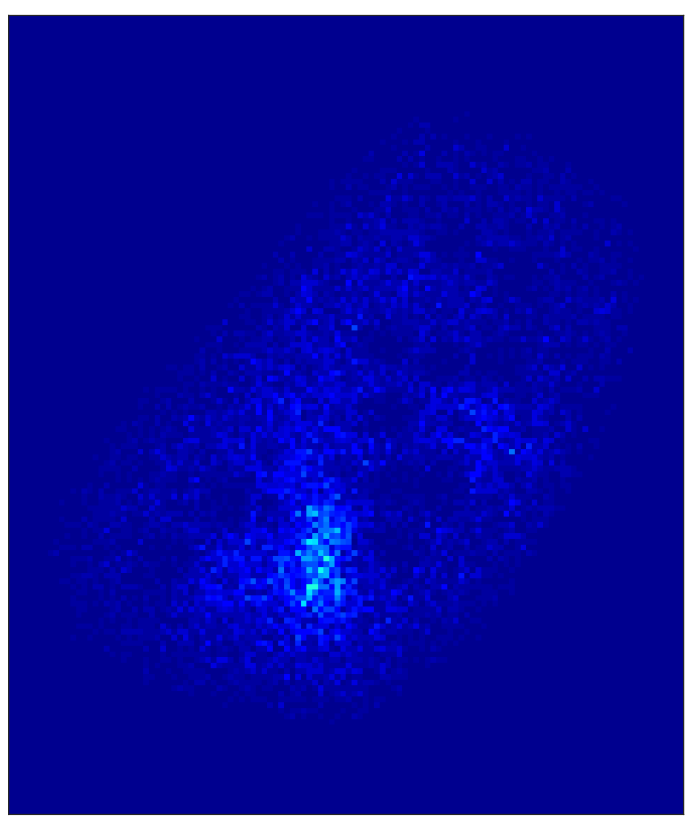

HN-SP
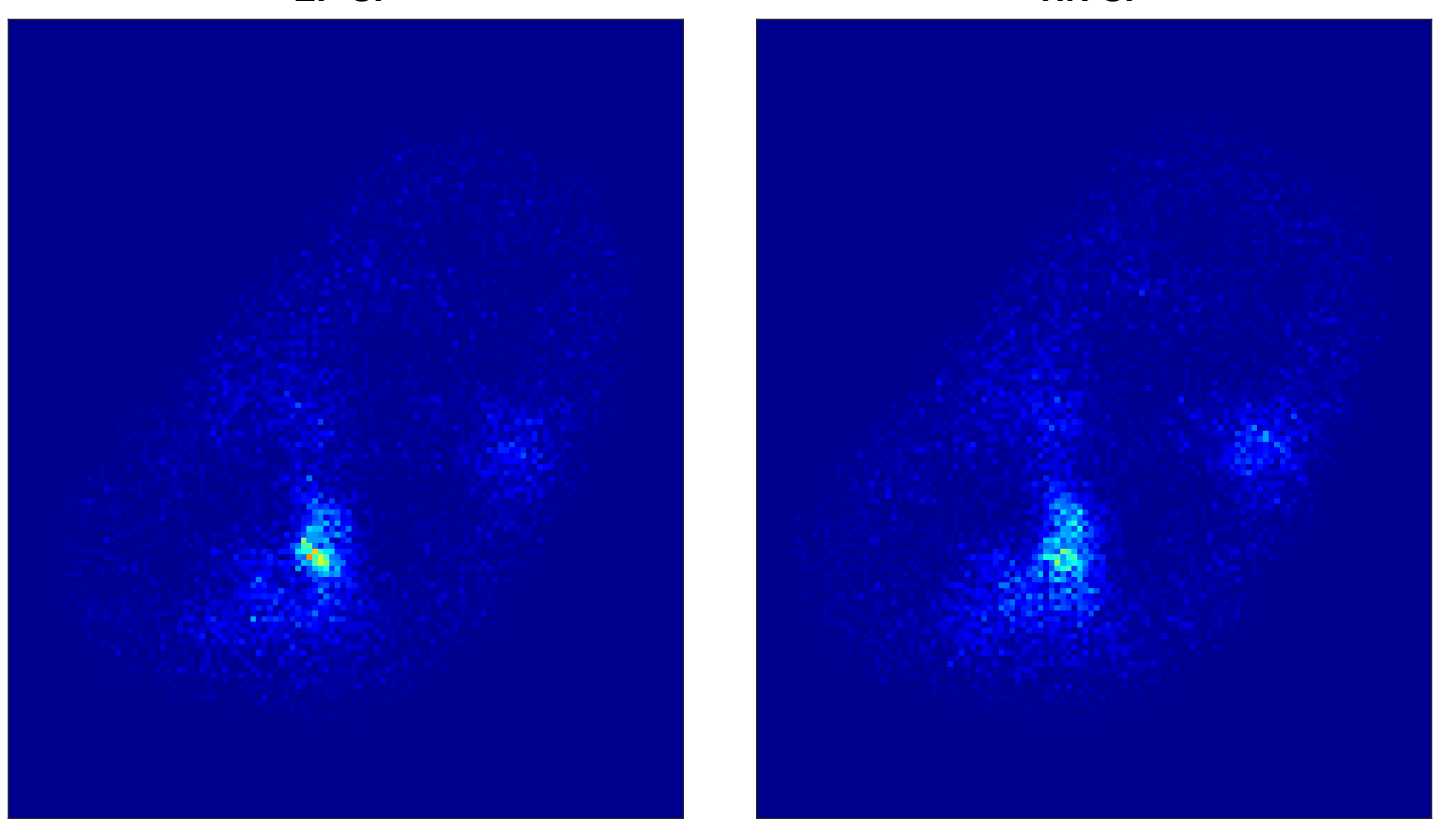


\section{CT Session S6 Home Range Center Density Plots}

EP

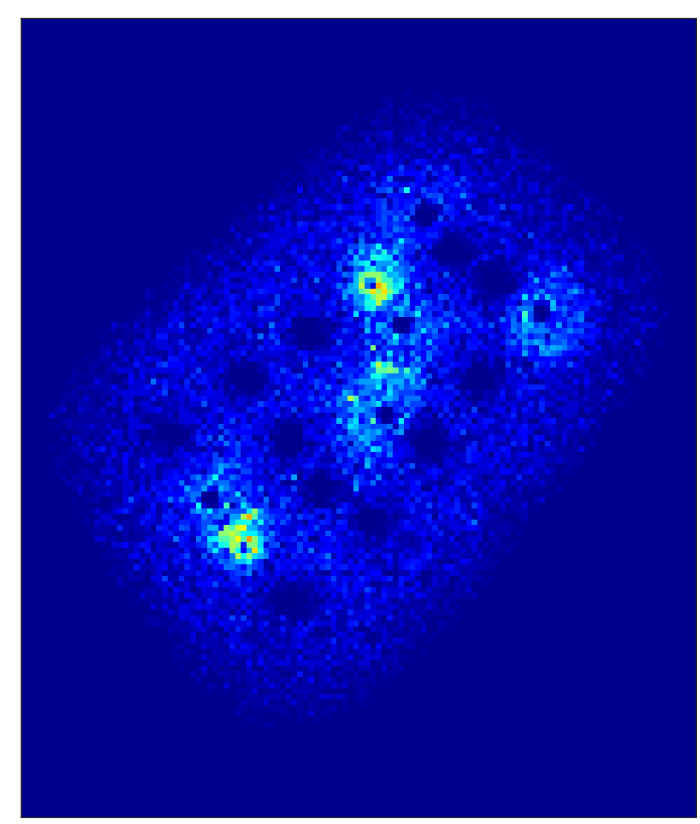

EP-SP

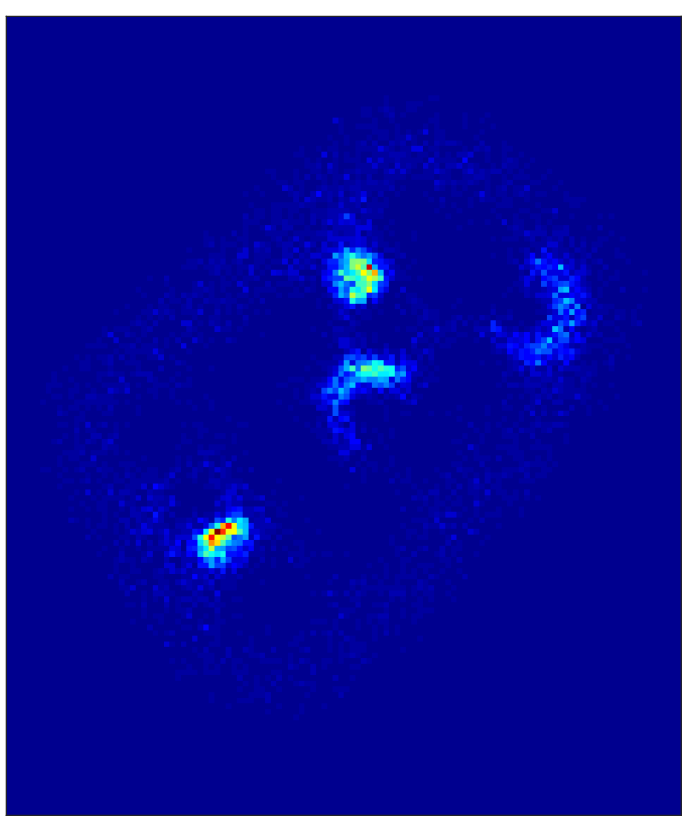

HN

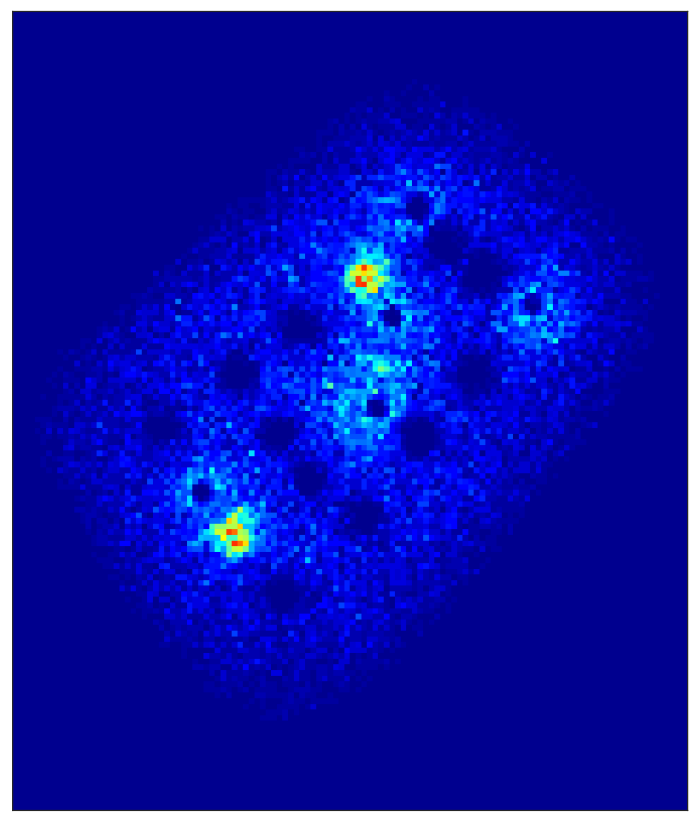

HN-SP

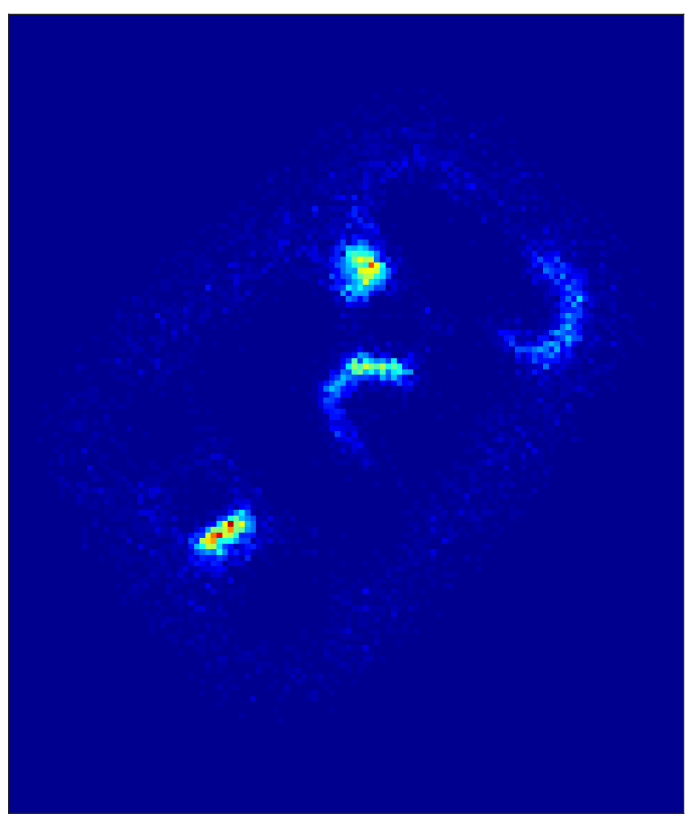


CT Session S7 Home Range Center Density Plots

EP

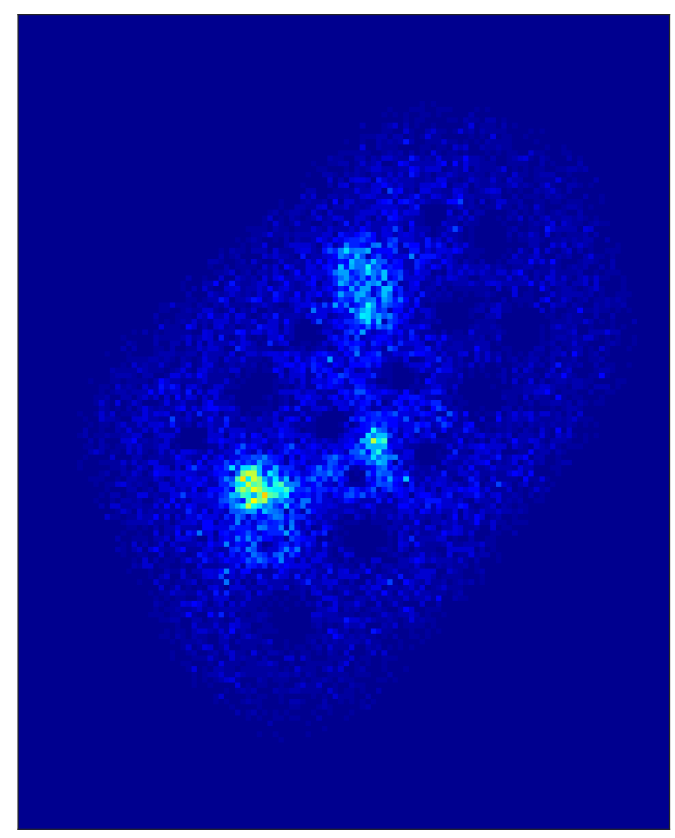

EP-SP

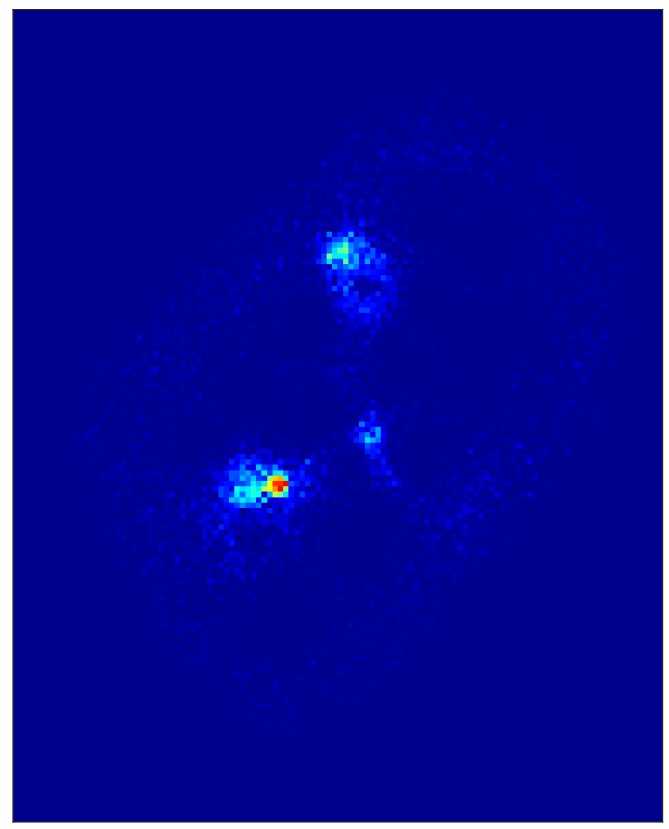

HN

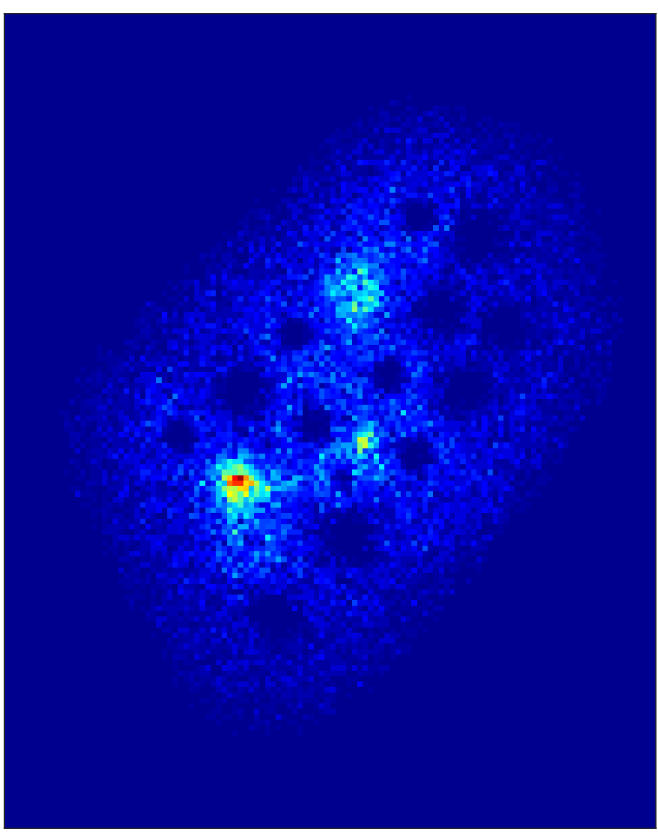

HN-SP

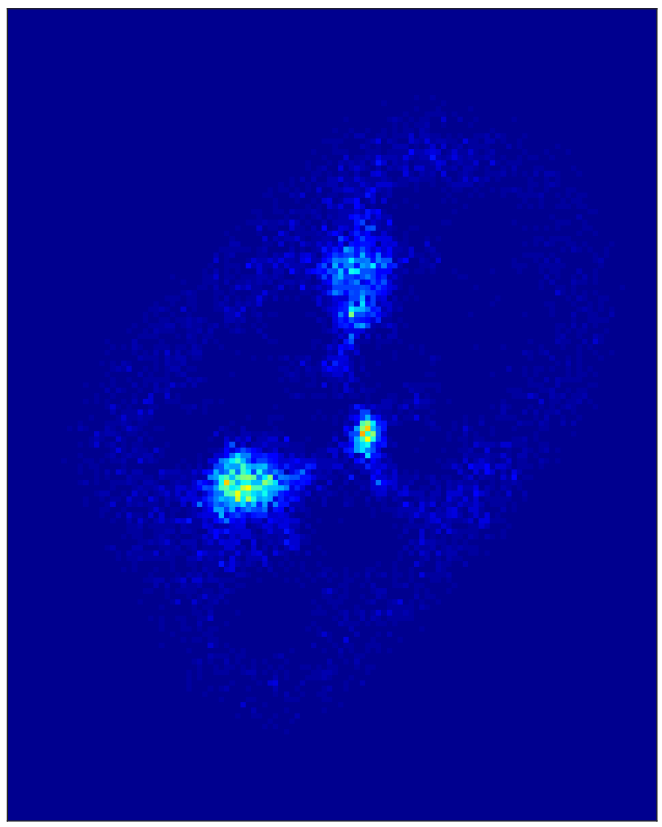


CT Session S8 Home Range Center Density Plots

EP

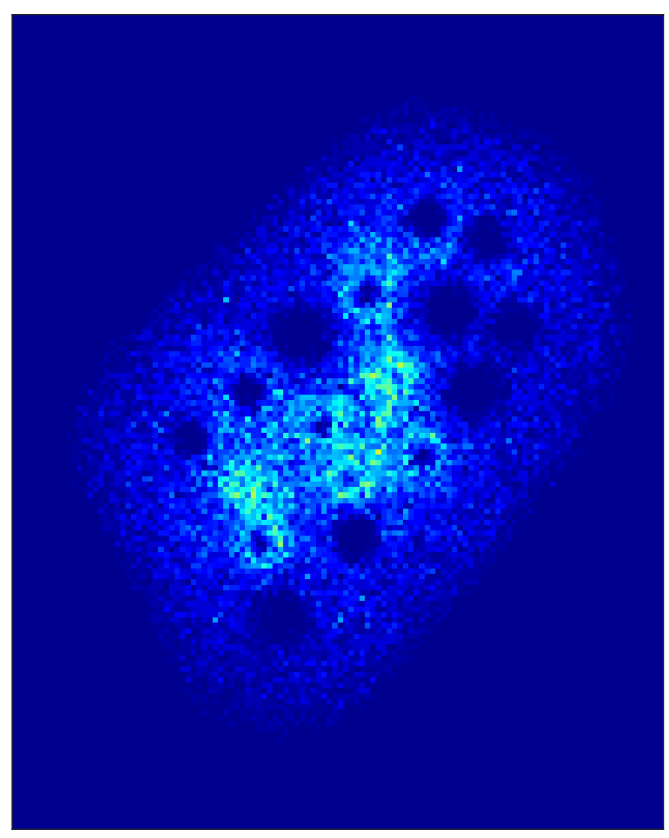

EP-SP

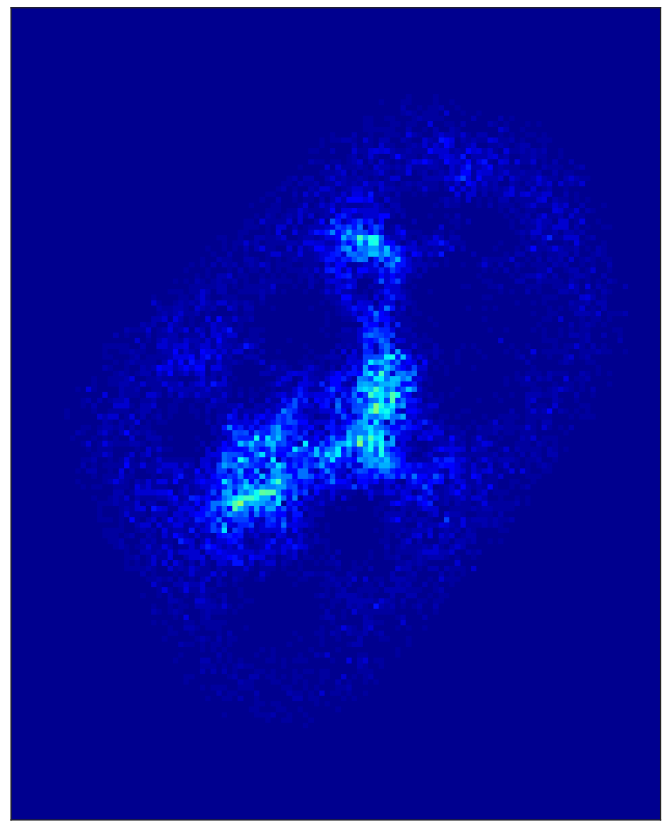

HN

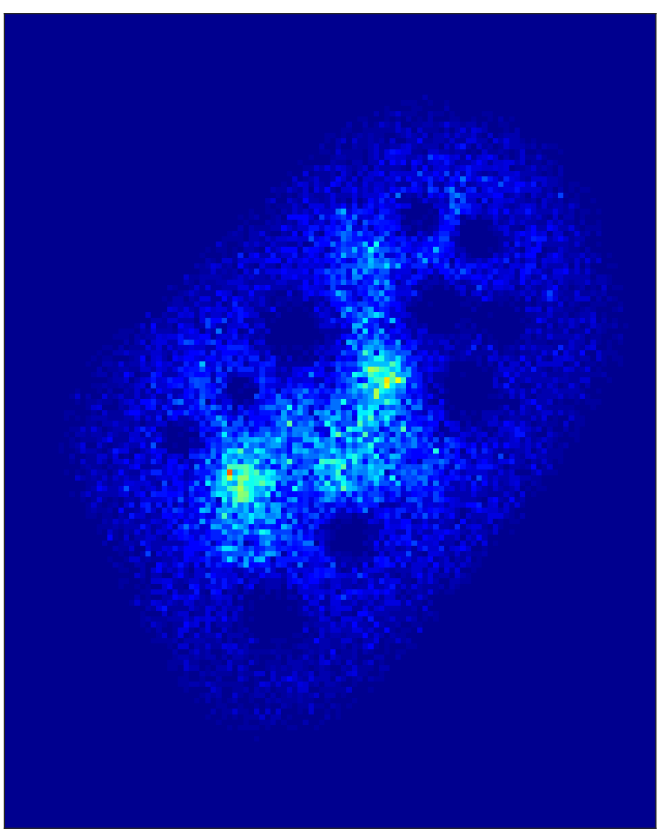

HN-SP

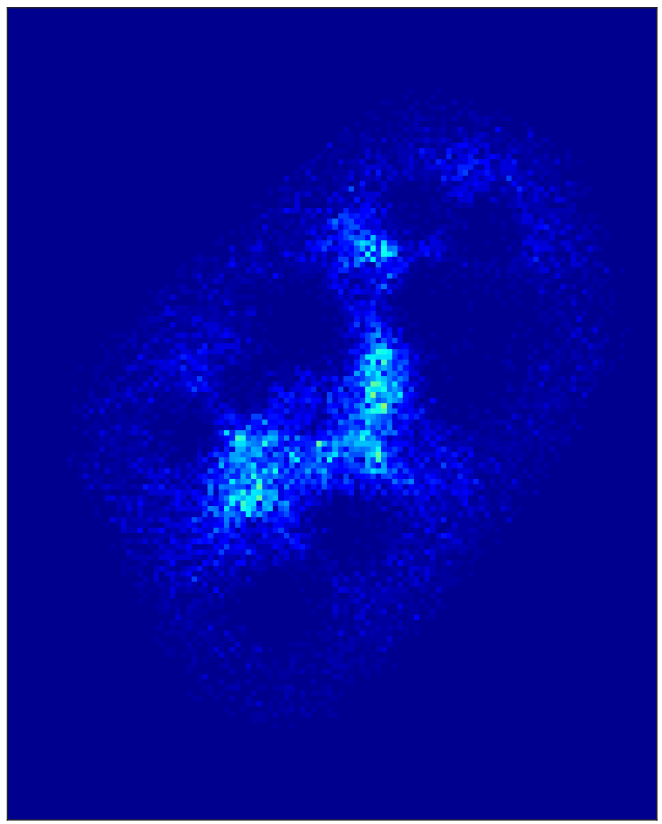




\section{CT Session S9 Home Range Center Density Plots}

EP

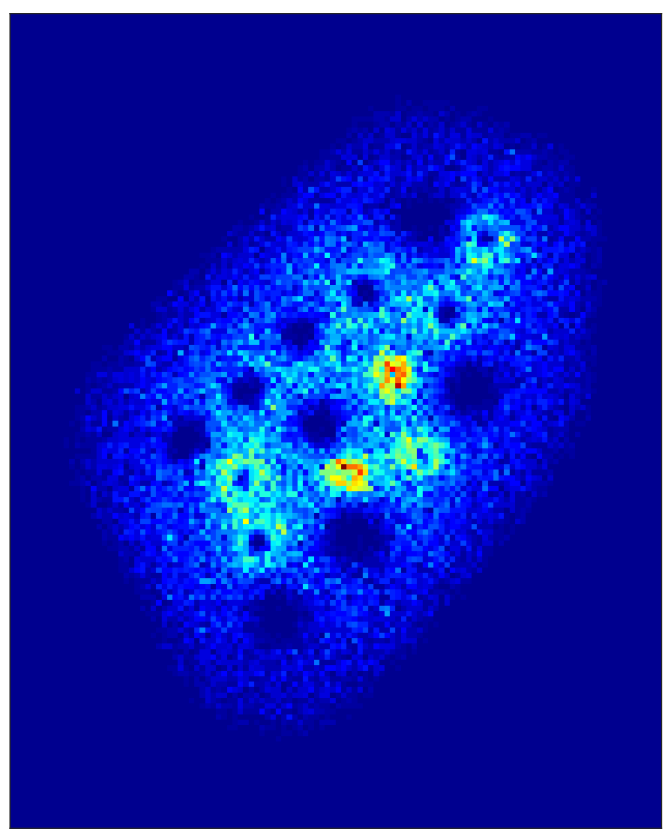

EP-SP

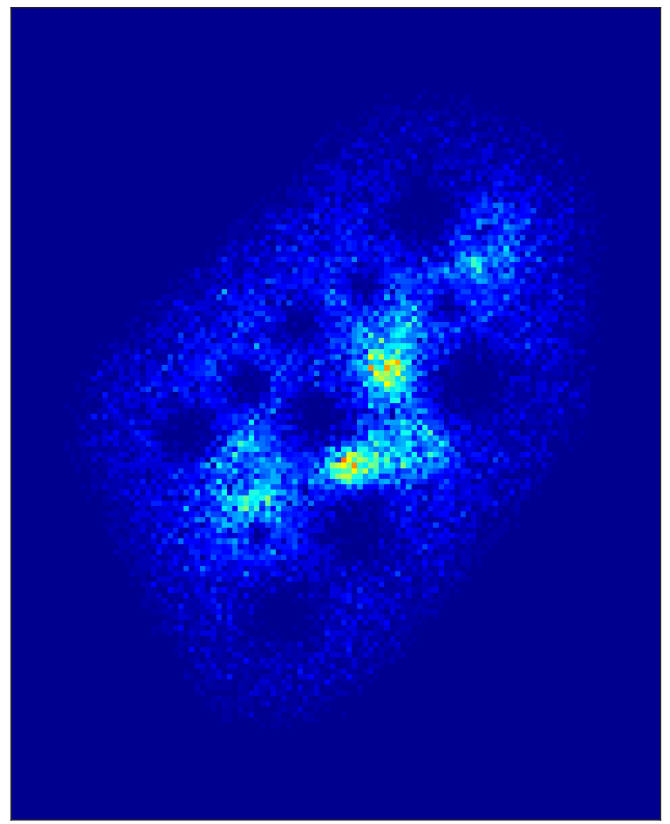

HN

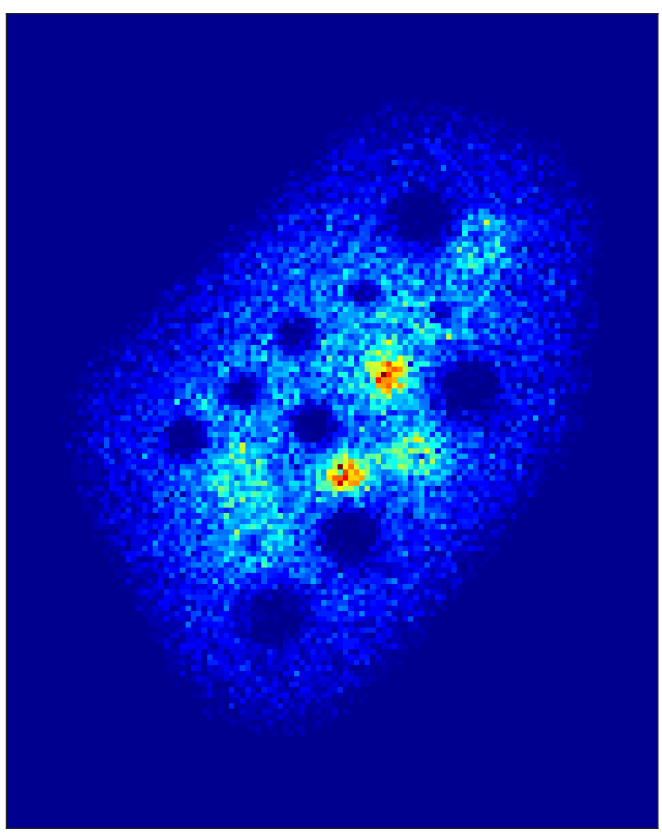

HN-SP

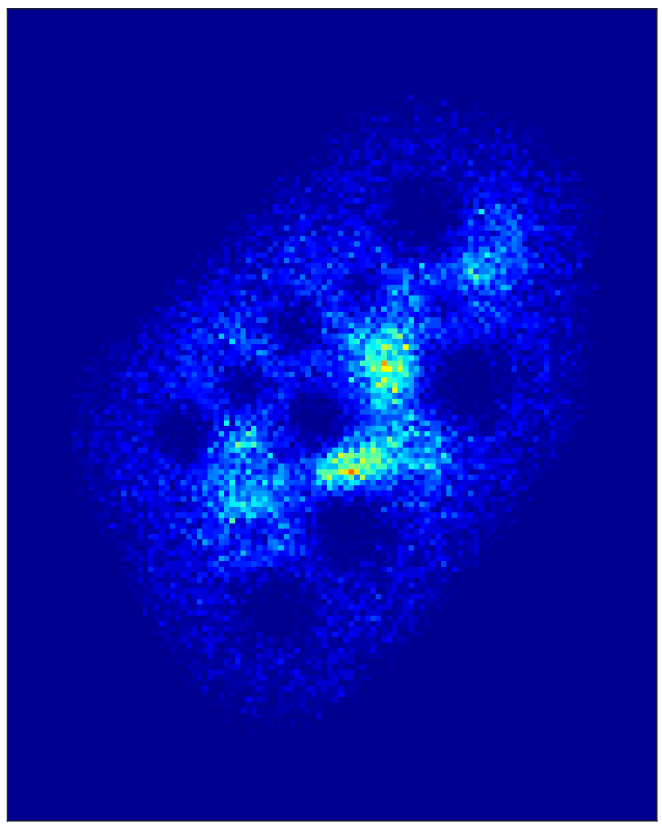


CT Session S10 Home Range Center Density Plots

EP

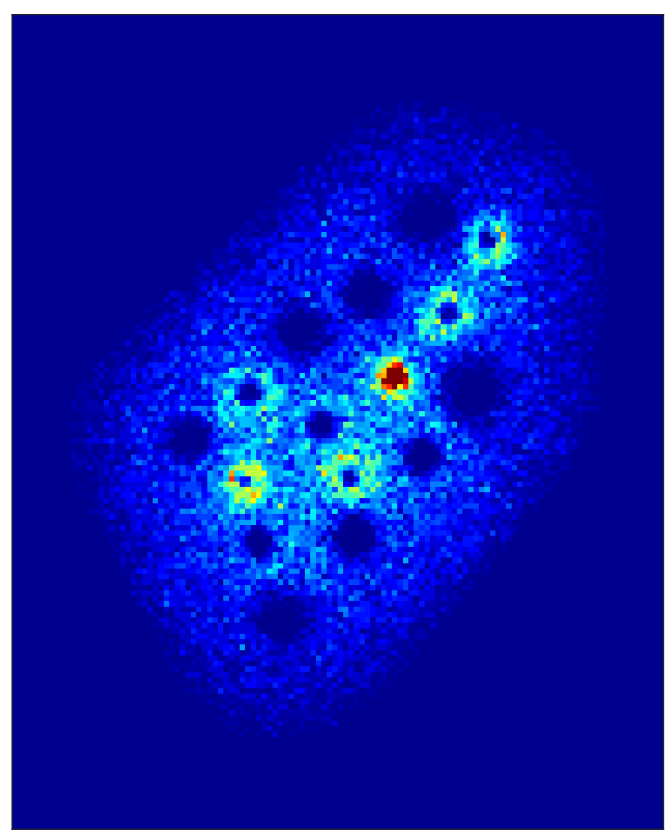

EP-SP

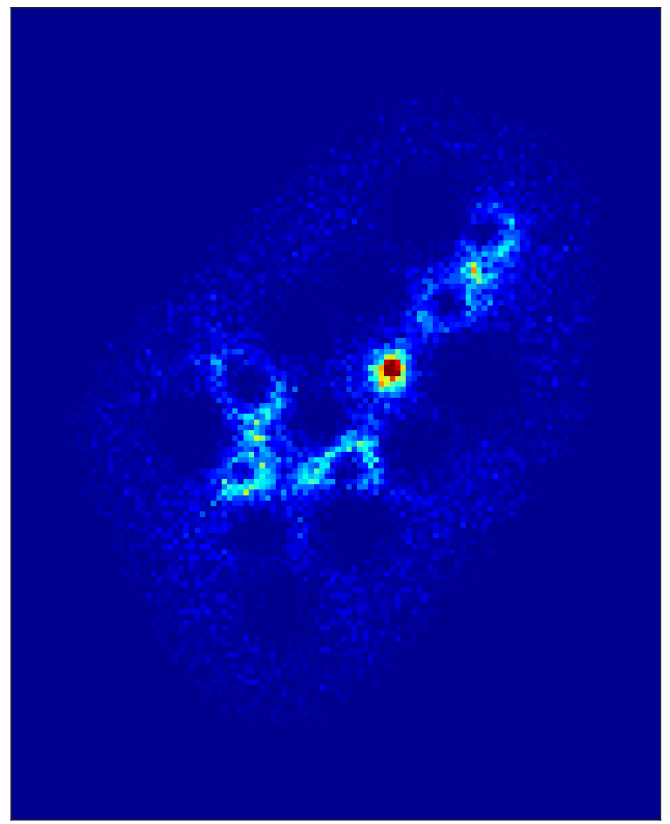

HN

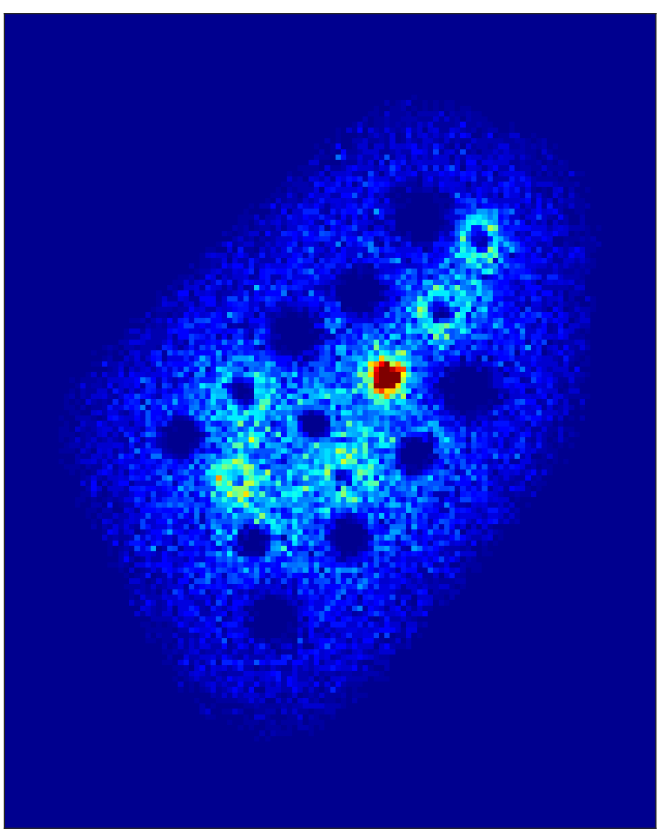

HN-SP

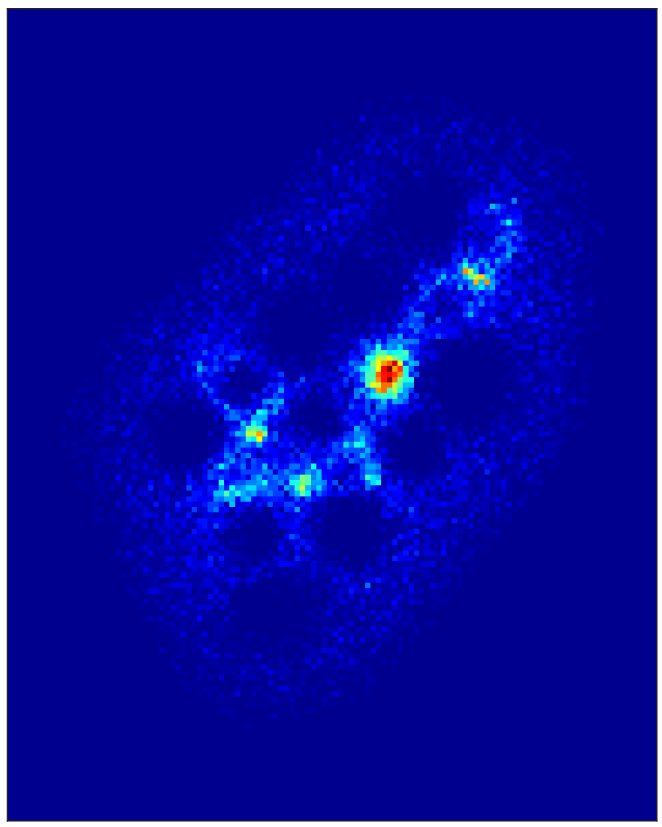


CTMix Session S1+S2 Home Range Center Density Plots

EP

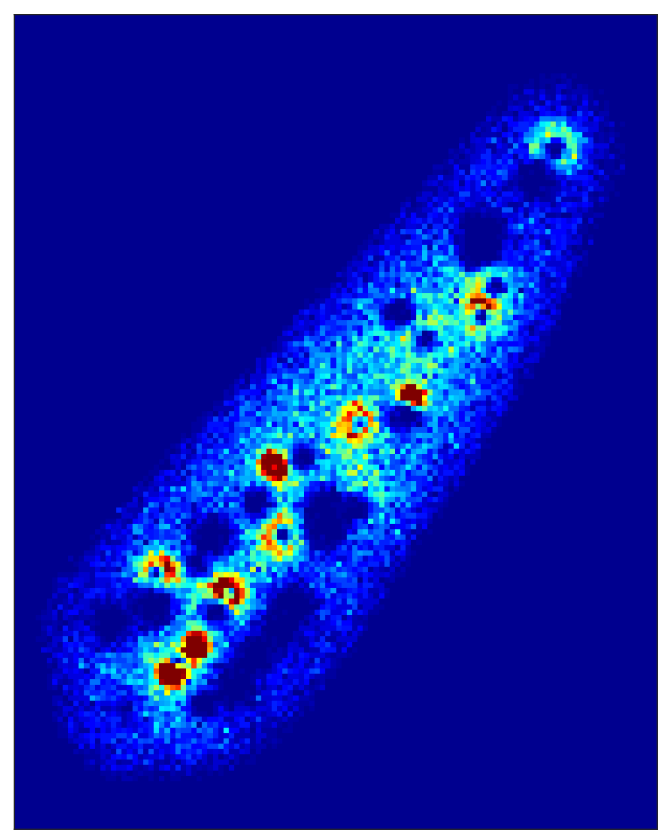

EP-SP

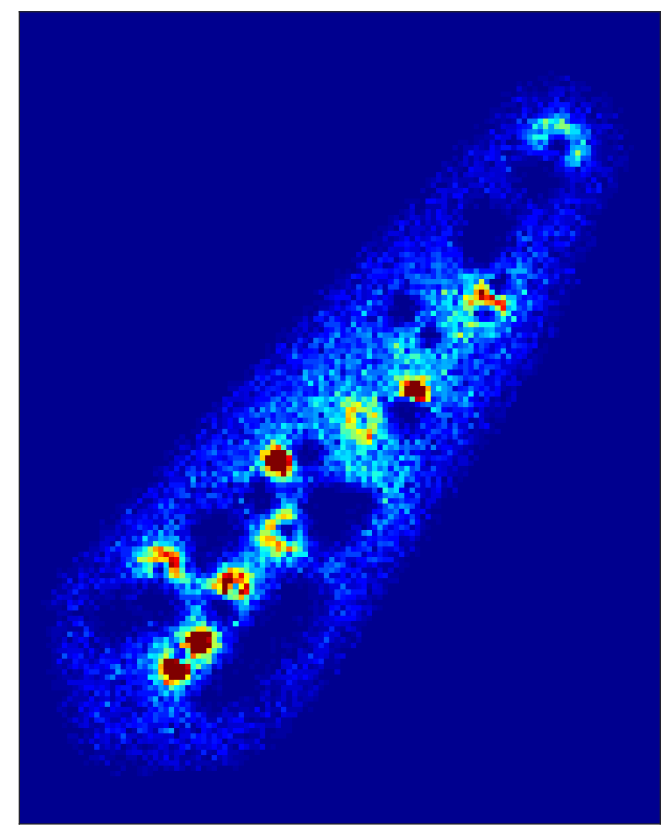

HN

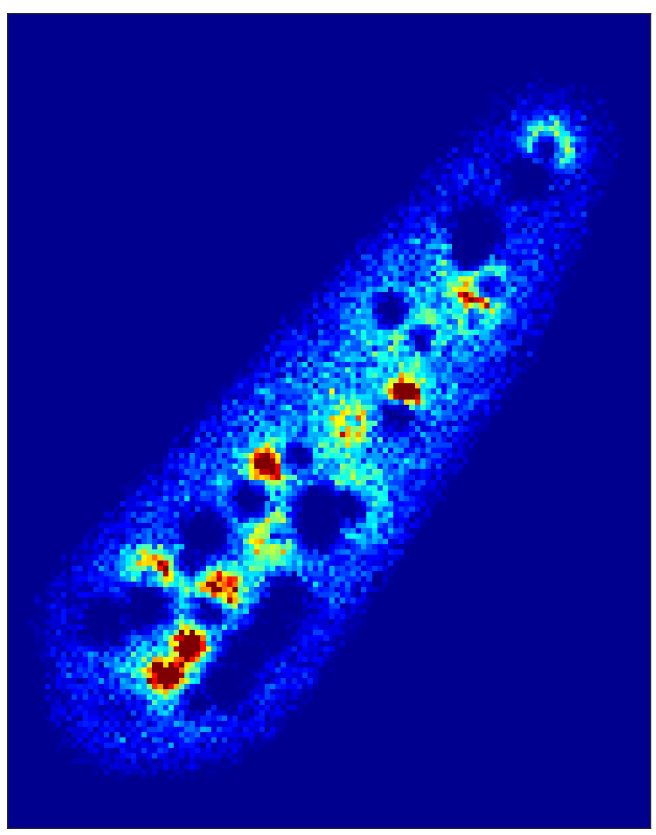

HN-SP

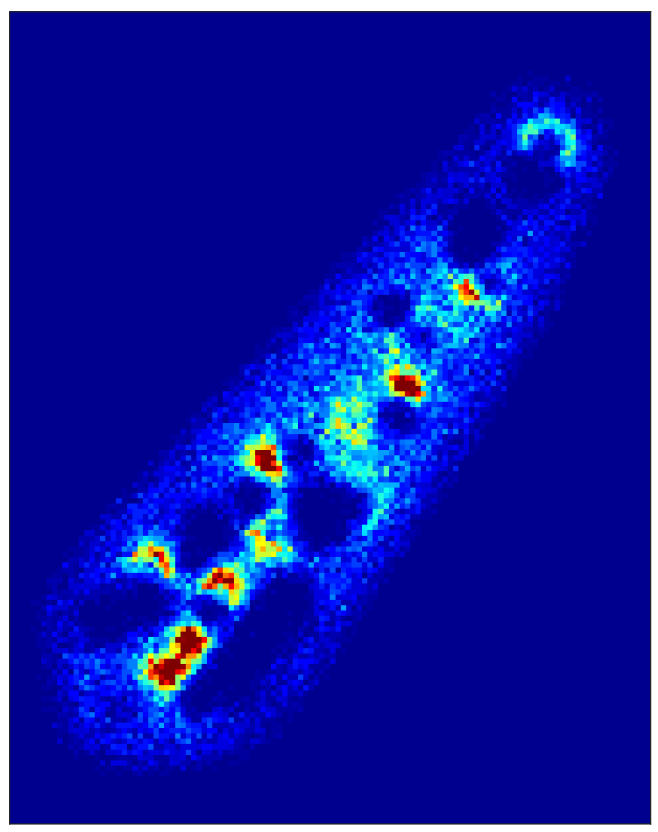


CTMix Session S3+S4 Home Range Center Density Plots

EP

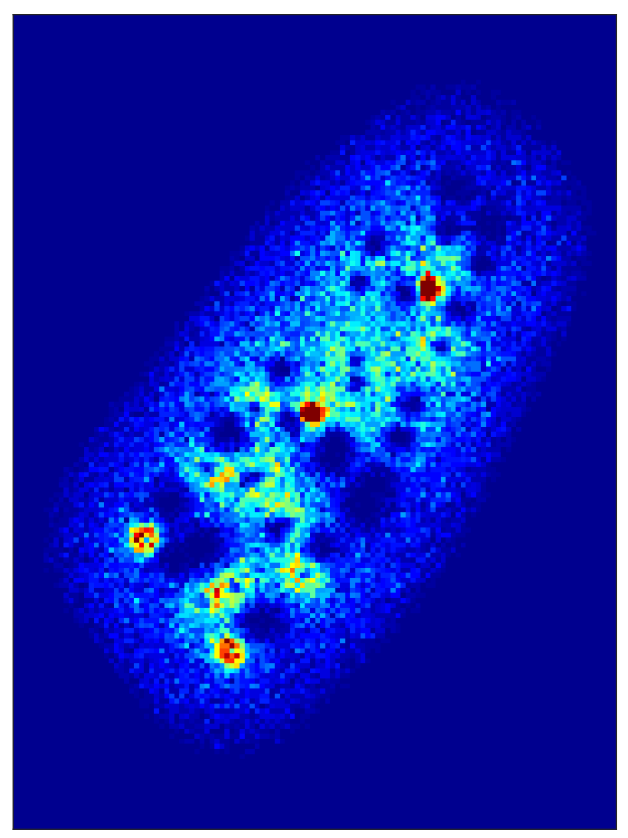

EP-SP

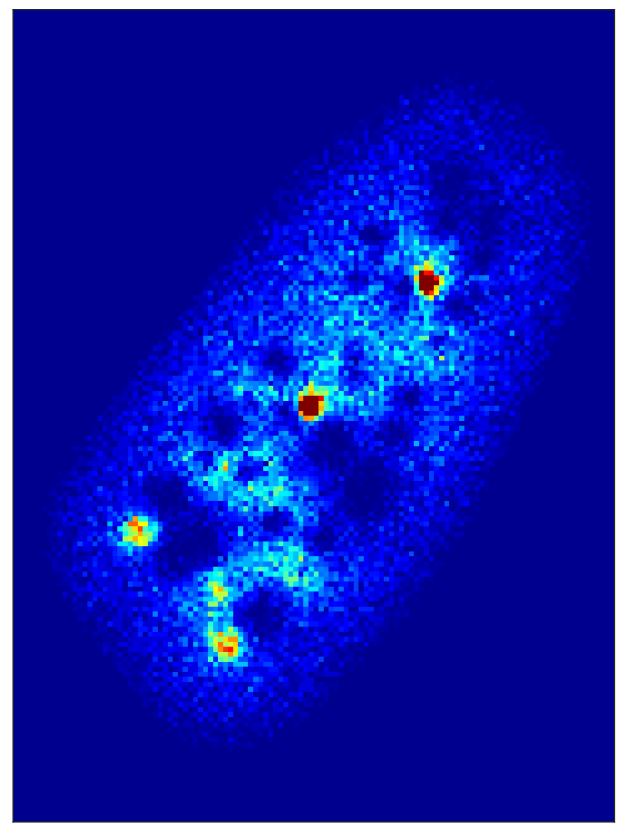

HN

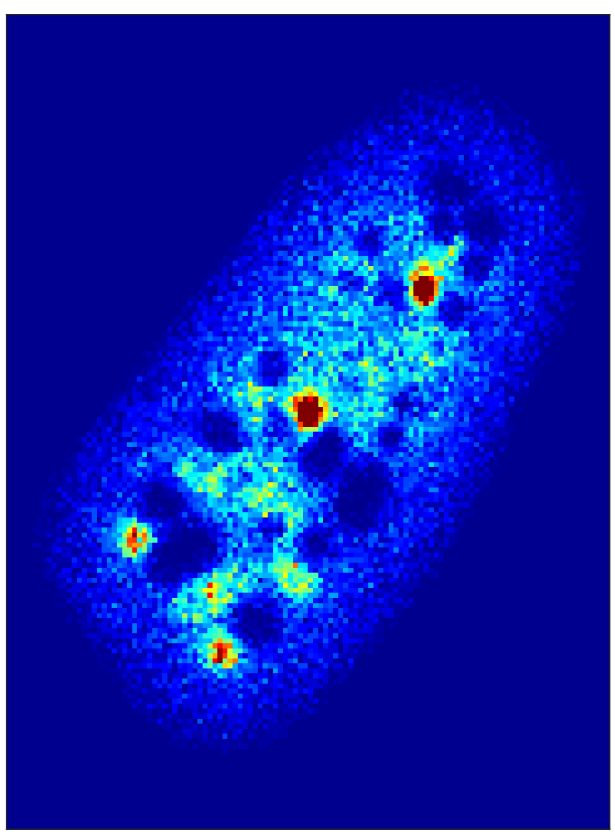

HN-SP

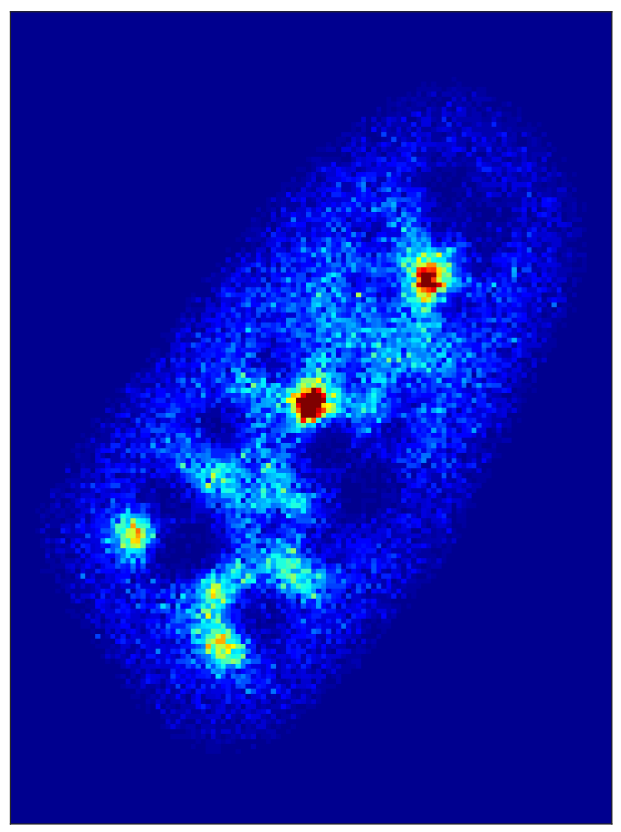


CTMix Session S4+S5 Home Range Center Density Plots

EP

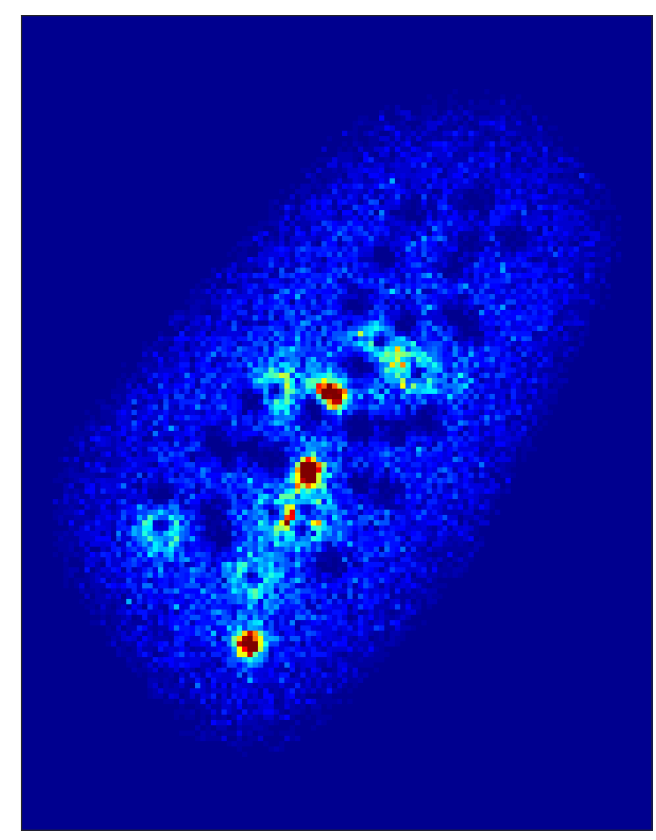

EP-SP

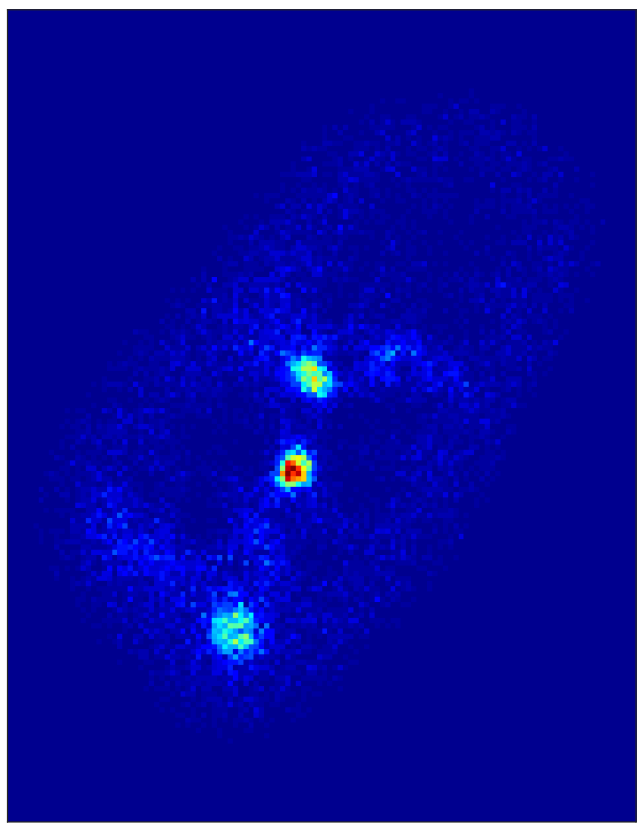

HN

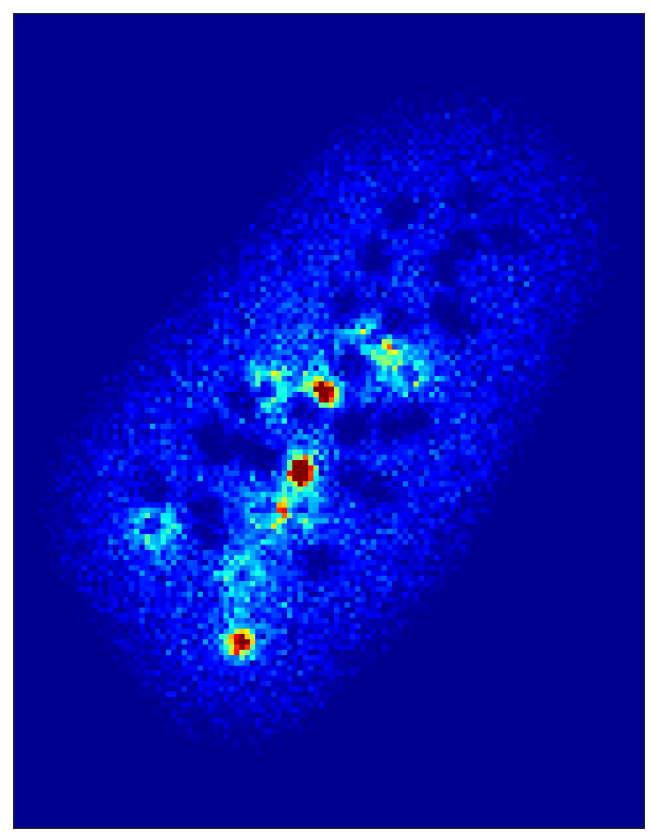

HN-SP

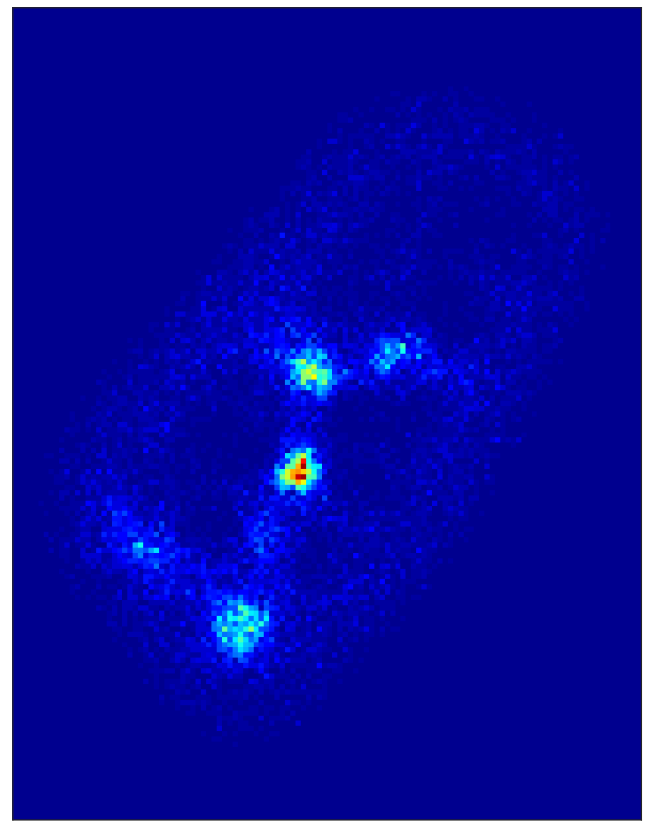


CTMix Session S5+S6 Home Range Center Density Plots

EP

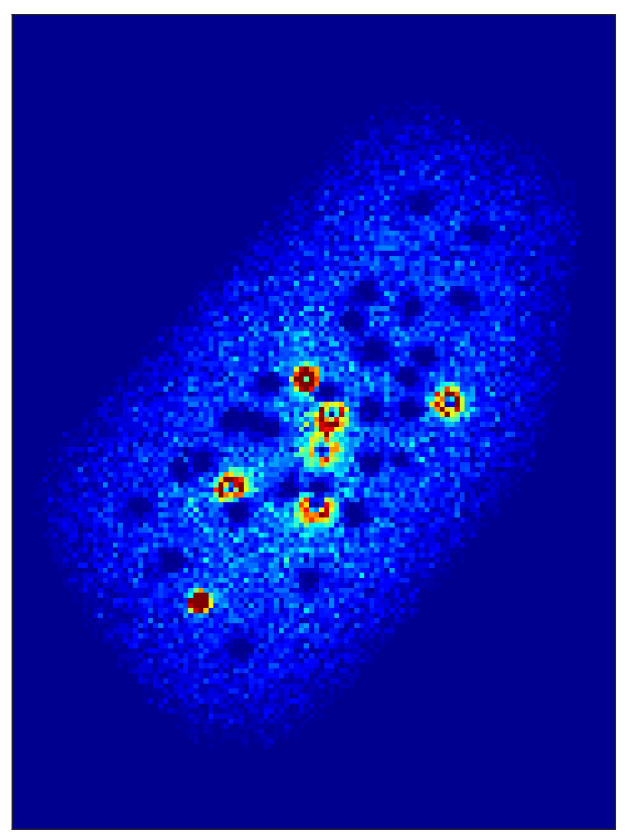

EP-SP

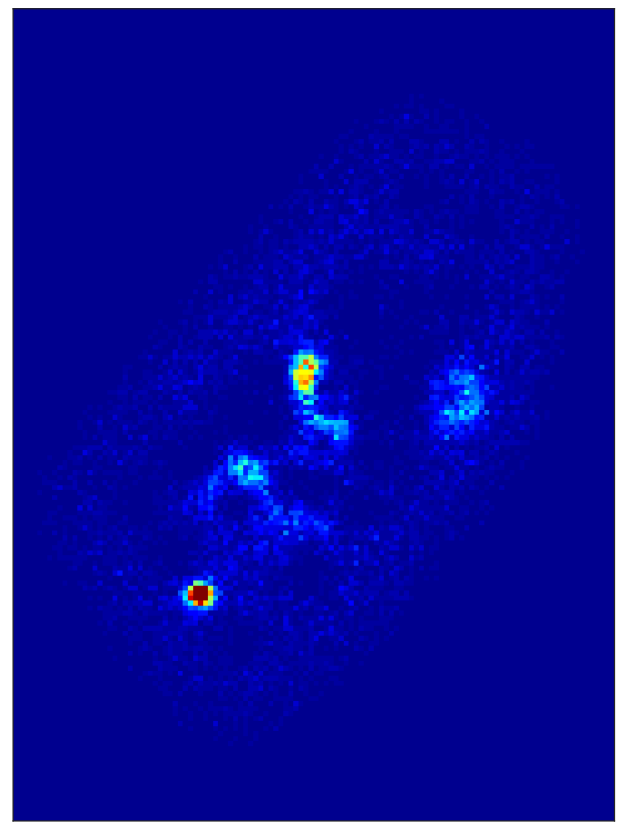

HN

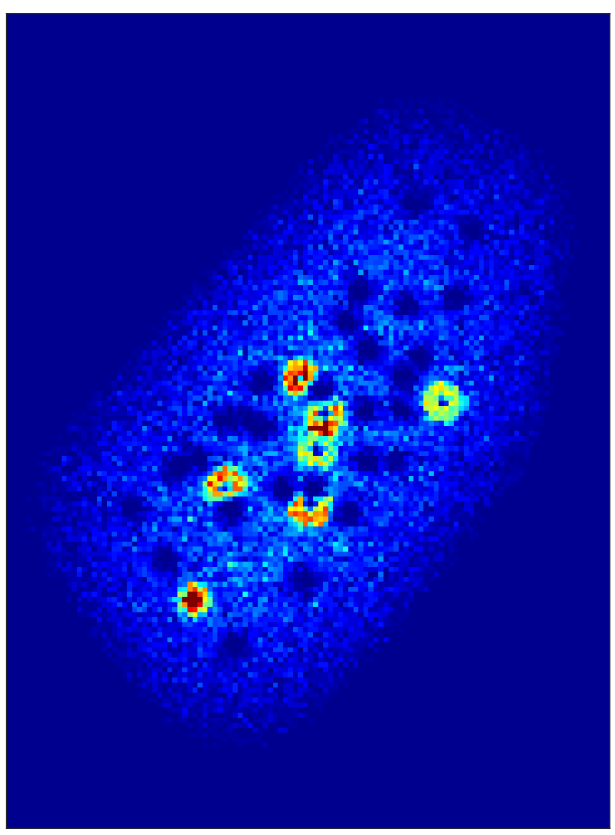

HN-SP

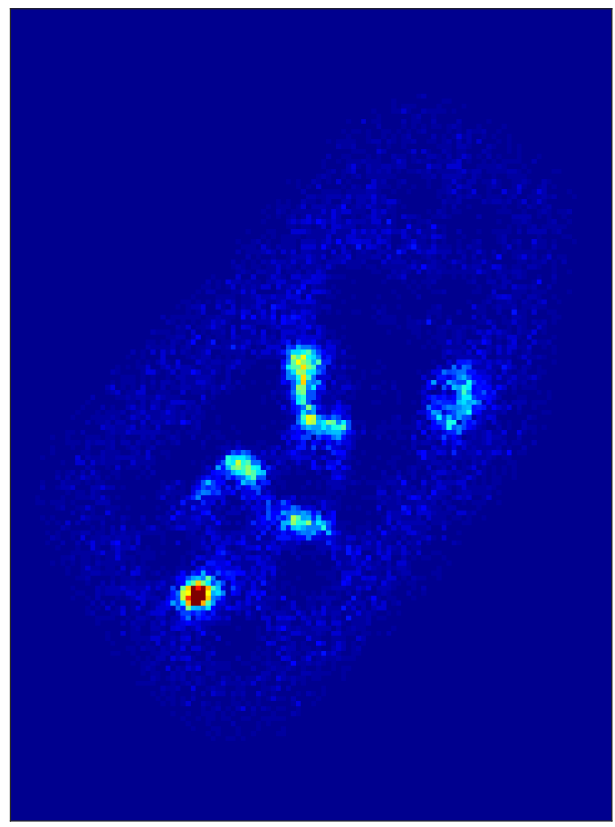


CTMix Session S6+S7 Home Range Center Density Plots

EP

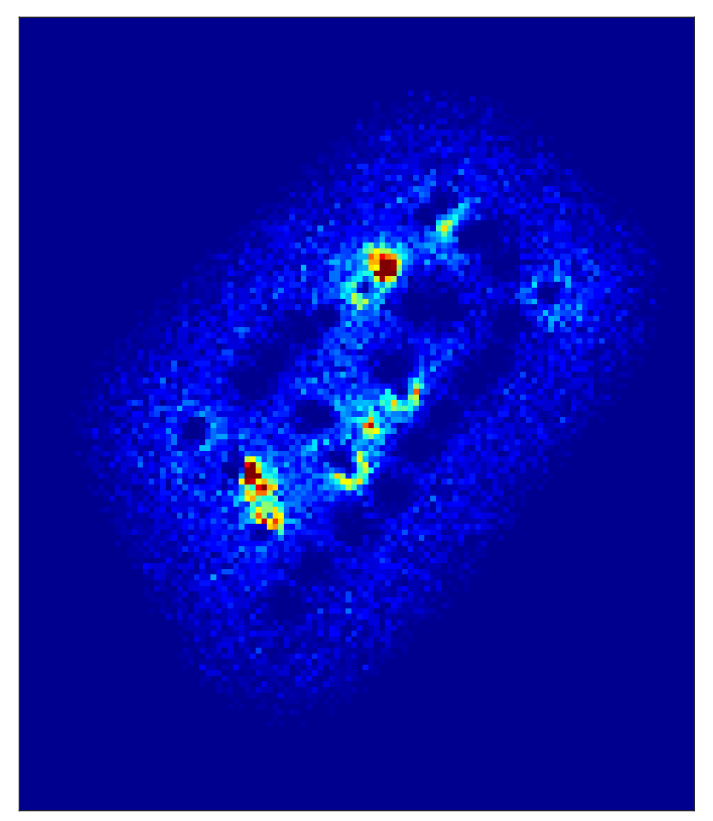

EP-SP

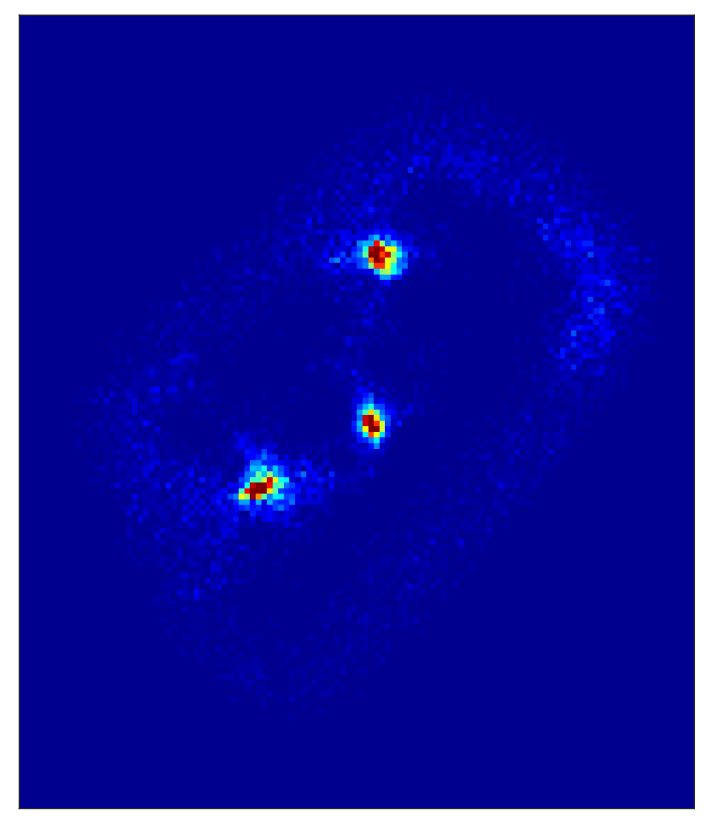

HN

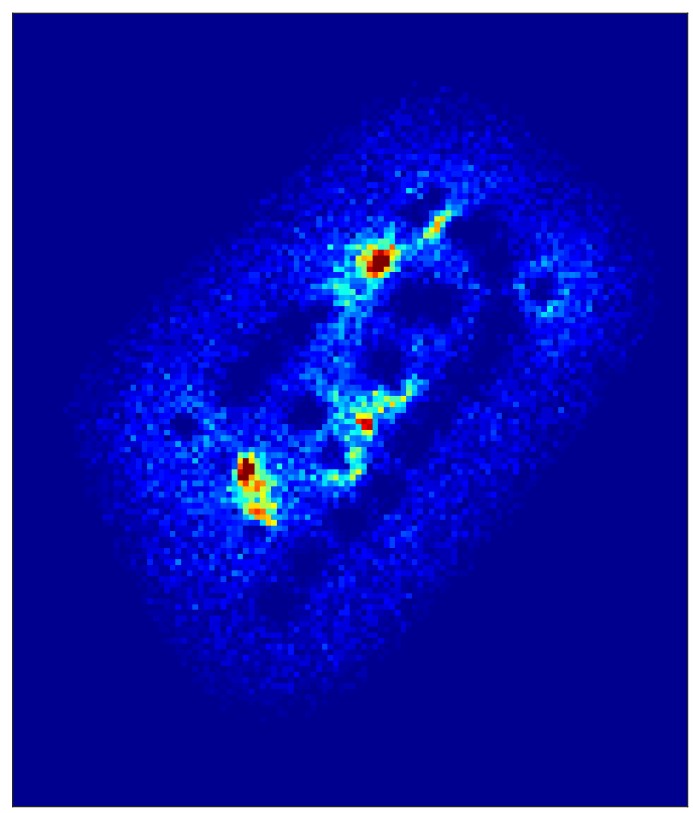

HN-SP

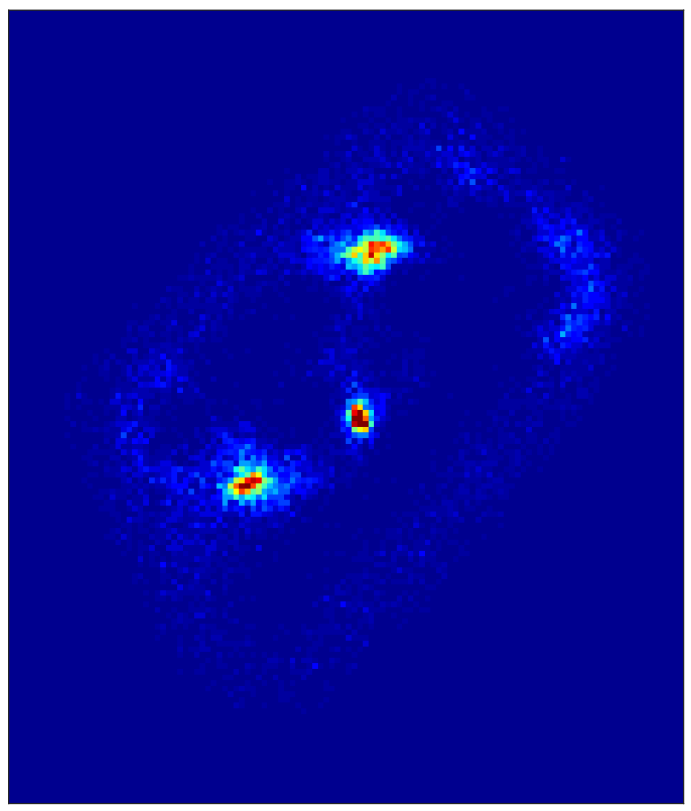

